\title{
Gestalttherapie zur Prävention von Depression
}

\author{
Ein Programm zur Gestaltgruppenarbeit \\ mit kreativen Medien für Jugendliche in der Schule
}

\author{
Dissertation \\ zur Erlangung des Doktorgrades \\ der Sozialwissenschaftlichen Fakultät \\ der Georg-August-Universität Göttingen \\ vorgelegt von \\ Jeong Sook Lee \\ geboren in Gangneung, Südkorea
}

Göttingen 2007 
1. Gutachterin: Prof. Dr. Christina Krause

2. Gutachter: Prof. Dr. Olaf-Axel Burow

Tag der mündlichen Prüfung: 19. Dez. 2007 


\section{Inhaltverzeichnis}

Abbildungsverzeichnis

Tabellenverzeichnis

Abkürzungsverzeichnis

Übersetzung von englischen Fachbegriffen

Danksagung

Abstract

Einleitung 1

\section{I .Teil: Theorie}

1 Depression bei Jugendlichen -----------------------------------------------------4

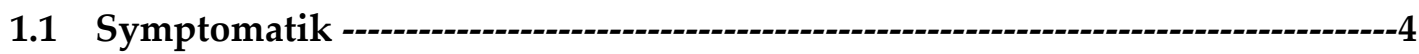

1.2 Merkmale der Depression im Jugendalter----------------------------------------5

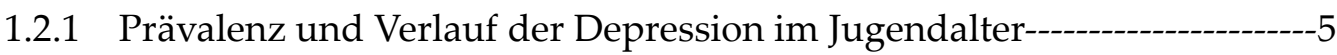

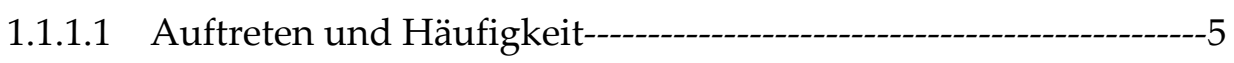

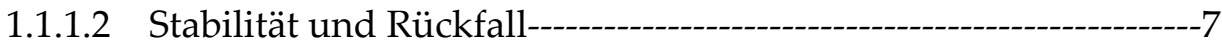

1.2.2 psychosoziale Beeinträchtigung---------------------------------------------7

1.2.3 Risikofaktoren für depressive Störungen im Jugendalter-------------------8

1.3 Schwierigkeiten früher Behandlung von Depression bei Jugendlichen-----10

1.4 Präventionsforschung von Depressionen im Jugendalter---------------------11

1.5 Probleme bei der Programmentwicklung für Depressionsprävention -------12

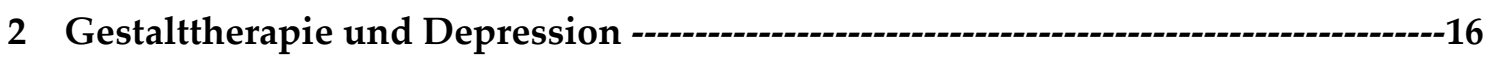

2.1 Zum Verstehen der Depression in gestalttherapeutischer Perspektive-------16

2.1.1 Depression und Kontaktunterbrechung-----------------------------------16

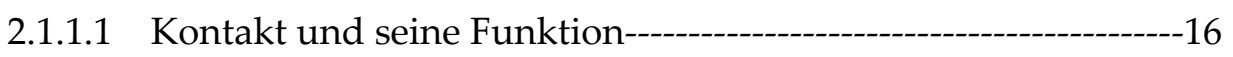

2.1.1.2 Depressive Prozesse und Kontaktunterbrechung----------------20

2.1.1.3 Depression als Polaritätskonflikt, als eingeschränkte Ich -Funktion oder als der zurückgedrängte expressive Impuls--------------27

2.1.2 Depression als dysfunktionale sekundäre Emotion aus der Perspektive

Emotion - Focused Therapy nach Greenberg und seiner Mitarbeiter-------31

2.1.2.1 Der Ansatz EFT und emotionale Schemata-------------------31

2.2.2.2 Depression als sekundäre emotionale Prozesse------------------34 
2.2 Die gestaltherapeutische Behandlung von Depressionen-----------------------37

2.2.1 Merkmale der Behandlung von Depressionen------------------------------37

2.2.1.1 Erfahrungsorientierte und emotionfokussierte Behandlung „Hier

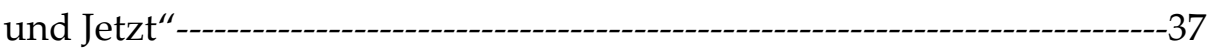

2.2.1.2 Verwendung der „Stuhltechnik“---------------------------------------42

2.2.2 Ergebnisse der Behandlung von Depressionen----------------------------50

2.2.3 Berücksichtigung der gestaltorientierten Behadlung von Jugendlichen bei Depressionen $-50$

3 Einsatz kreativer Medien in der Gestalttherapie-------------------------------55

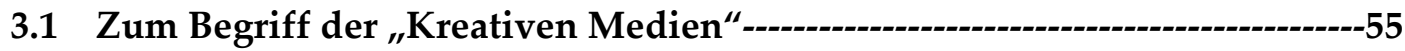

3.2 Gestaltpsychologische Theorie und der Einsatz kreativer Medien-----------58

3.2.1 Ganzheitstheorie-------

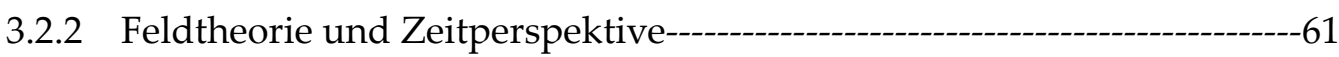

3.2.3 Phänomenologie zur Gestaltbildung--------------------------------------63

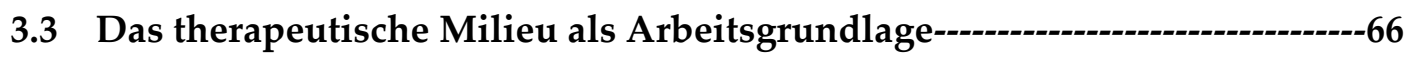

3.3.1 Förderung der Kreativität---------------------------------------------------------66

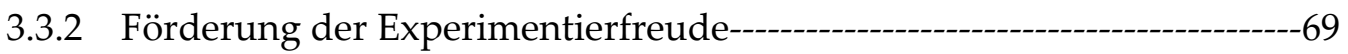

3.4 Chance und Grenzen der Verwendung kreativer Medien in bestehenden

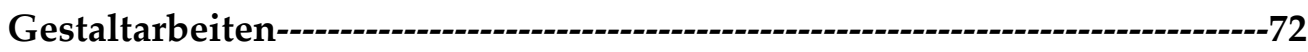

3.5 Wirkungspotentiale kreativer Medien in der Gestaltarbeit---------------------75

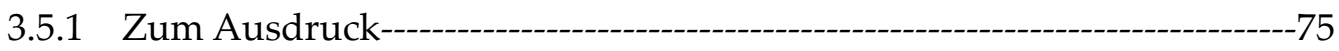

3.5.1.1 Katalysator zur Wahrnehmungserweiterung--------------------75

3.5.1.2 Multidimensionales Ausdrucksmittel------------------------------76

3.5.1.3 Ästhetisches Erfahrungsmittel------------------------------------77

3.5.2 Zur Erfahrungsanreicherung mit dem gestalteten Produkt----------------78

3.5.2.1 Der Zugang zum „eigenen archaischen Land“ des Klienten---$-78$

3.5.2.2 Das Mittel der dialogischen Selbstreflexion---------------------79

3.5.2.3 Die Boje zum neuen Anfang----------------------------------------80

3.5.3 Zur Diagnostik und Informationserhebung---------------------------------81

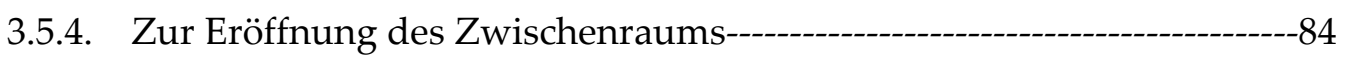




\section{Teil: Entwicklung, Anwendung und Evaluation des Programms zur indikativen Prävention von Depression}

4 Konzeptentwicklung für die Gestaltarbeit mit kreativen Medien bei depressiven Jugendlichen---------------------------------------------------------86

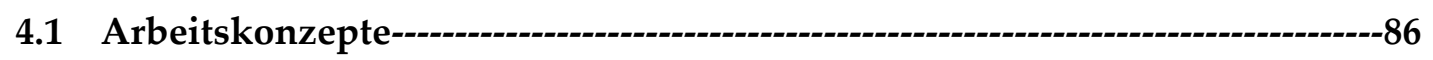

4.1.1 Entwicklungspsychologische Perspektive-----------------------------------86

4.1.2 Gruppenarbeitssetting--------------------------------------------88

4.1.3 Erlebnisfördernde und ausdruckorientierte Modalitäten------------------90

4.1.4 Einsatz des gestealteten Produkts statt des Stuhls--------------------------91

4.1.5 Haltung des Therapeuten oder Beraters-------------------------------------93

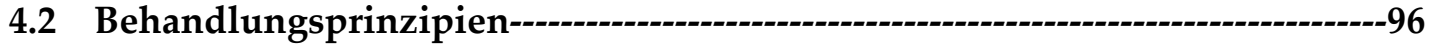

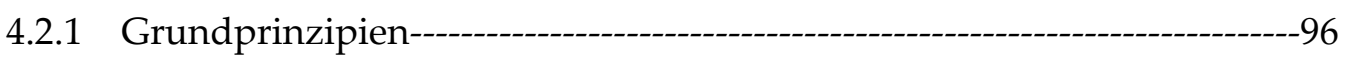

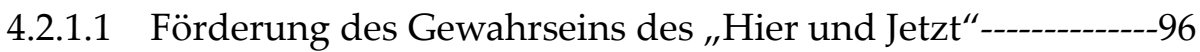

4.2.1.2 Phänomenologischer Vorgang---------------------------------98

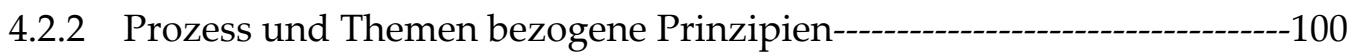

4.2.2.1 Arbeitsverlauf nach Prozess und Themen-----------------------100

4.2.2.2 Problemaktualisierung und Ressourcenaktivierung im ersten

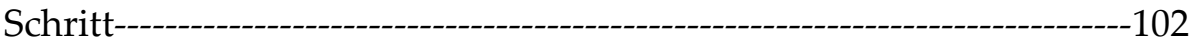

4.2.2.3 Problembewältigung, Auflösung der Konflikte und unterbrochene Prozesse oder Gefühle im zweiten Schritt---------------105

4.2.2.4 Stärkung zur Selbstunterstützung und Gewinnung eigenen

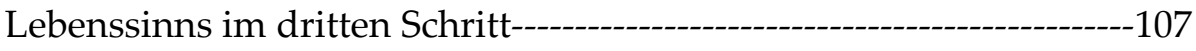

4.3 Therapeutische Phasen der Arbeit mit kreativen Medien in der Sitzungen----

4.3.1 Phase 1: Der Aufbau---_-110

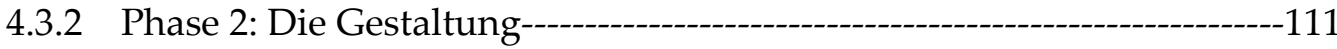

4.3.3 Phase 3: Die Intensität------_---113

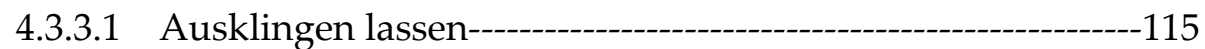

4.3.3.2 Begegnung-----------------------------------------------------------116

4.3.4 Phase 4: Die Zusammenführung und Eröffnung neuer Wachstumsdimensionen$-117$

5 Untersuchungsziel und Hypothesen- 


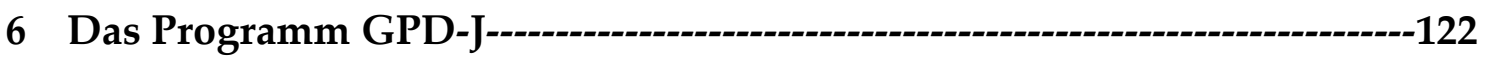

6.1 Design des Programms GPD-J-------122

6.1.1 Ziel---122

6.1.2 Zielgruppe und Ein- sowie Ausschlusskriterien -----------------------122

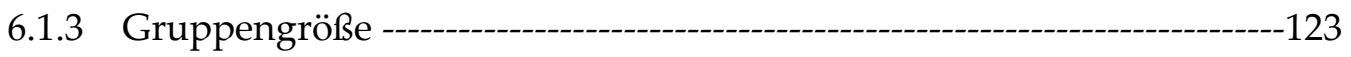

6.2 Struktur des Programms GPD-J-123

6.2.1 Inhalte des Programms GPD-J-------------------------------123

6.2.2 Vorbereitung auf jede Sitzung -----_-

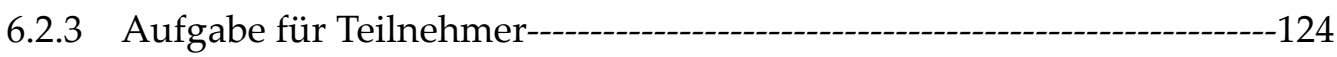

6.2.4 Prozessverlauf und Themen des Programms GPD-J--_-_-_-_--124

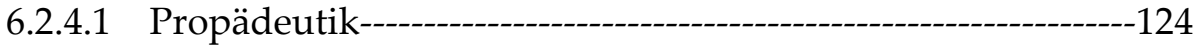

6.2.4.2 1. Sitzung: Selbstvorstellung----------------------------124

6.2.4.3 2. Sitzung: Familie als Lebensraum--_-124

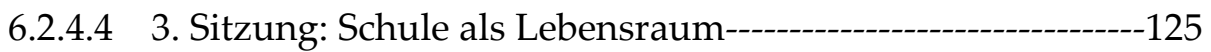

6.2.4.5 4. Sitzung: Negative Gefühle zum Selbst--------------------125

6.2.4.6 5. Sitzung: Ausdruck der negativen Gefühle zum Selbst-----126

6.2.4.7 6. Sitzung: Unterbrochene Gefühle zu einer bestimmten

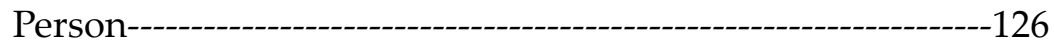

6.2.4.8 7. Sitzung: Ausdruck der unterbrochenen Gefühle zu einer bestimmten Person----_--126

6.2.4.9 8. Sitzung: Gestalten eigener Grenzen----------------------127

6.2.4.10 9. Sitzung: Ich bin Ich-------

6.2.4.11 10.Sitzung: Abschied---_-127

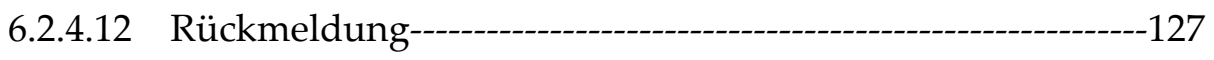

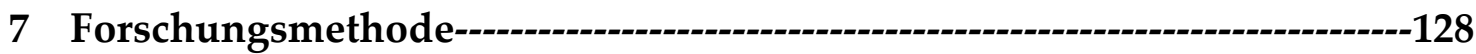

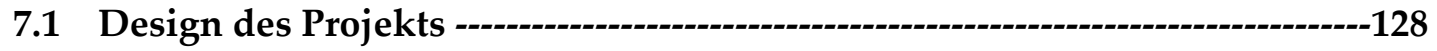

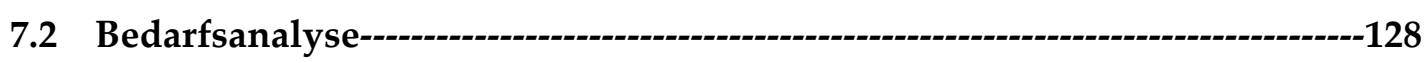

7.3 Auswahl der Teilnehmer und Gruppierung--130

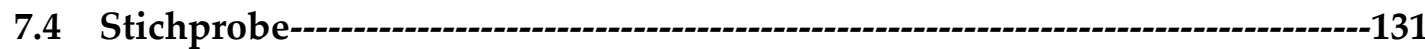

7.4.1 Demographische Daten---131

7.4.2 Gesundheit und Konsumverhalten----132

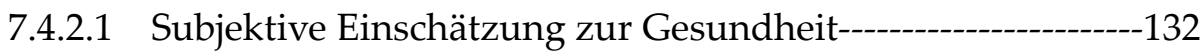

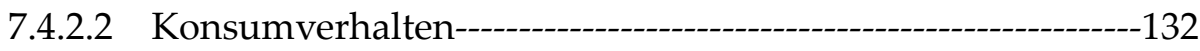

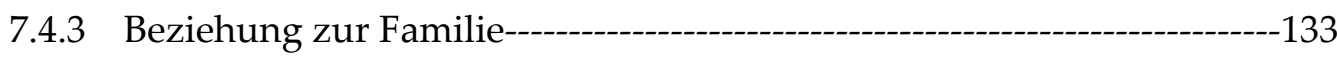

7.4.4 Stressor, körperliches Befinden und Bewältigungsstrategie ------------133 


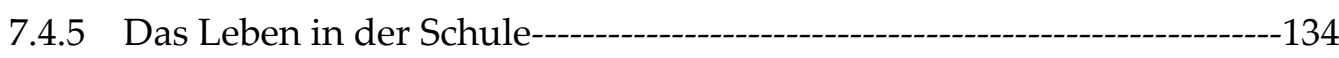

7.4.5.1 Grundstimmung in der Schule-------------------------------135

7.4.5.2 Allgemeine Zufriedenheit mit dem Leben in der Schule----135

7.4.5.3 Zufriedenheit in der Beziehung zu Schukameraden bzw. Lerhrern-----------------------------------------------------------------------135

7.4.6 Vorerfahrung in der Teilnahme an einer Psychotherapie oder einer psychologischen Beratung-----------------------------------------------------------135

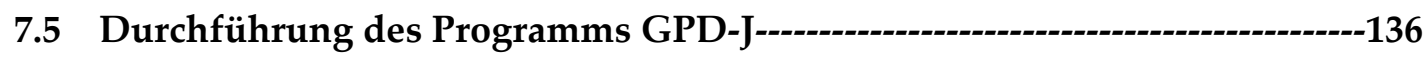

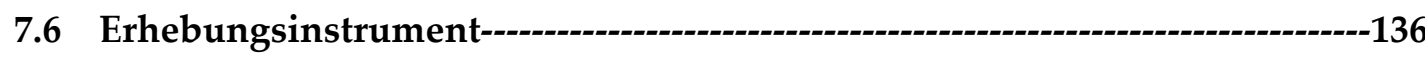

7.6.1 Zur Erhebung der Wirksamkeit des Programms GPD-J------------------137

7.6.1.1 Zur Erfassung depressiver Symptombelastung-----------------137

7.6.1.1.1 BDI (Becks Depression Inventory)-------------------137

7.6.1.1.2 Depressivität in der SCL-90-R----------------------137

7.6.1.2 Zur Erfassung allgemeiner psychischer Beschwerden: Die Symptomcheckliste(SCL-90 Revised)---------------------------------138

7.6.1.3 Zur Erfassung psychischen Wohbefindens : Psychological

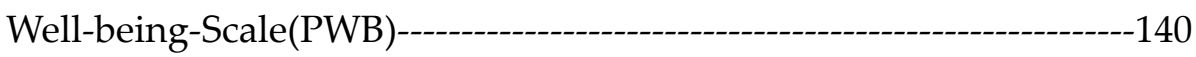

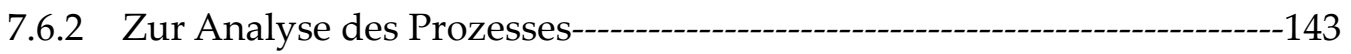

7.6.2.1 Verlaufsentwicklung der Depression während des Programms: BDI-----------------------------------------------------------------143

7.6.2.2 Das hilfreichste Erlebnis: Instrument für das hilfreichste Erlebnis (IHE)--------------------------------------------------------143

7.7 Messzeitpunkte----------------------------------------------------------------------------144

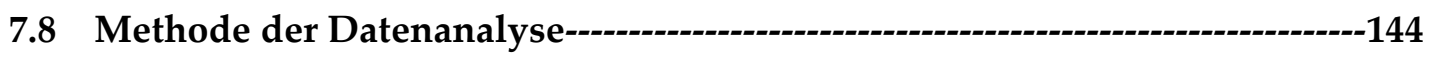

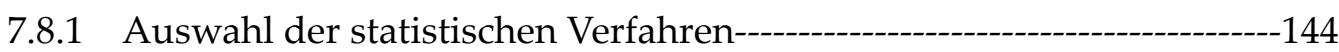

7.8.2 Zur Untersuchung des Therapieprozesses mit dem hilfreichsten

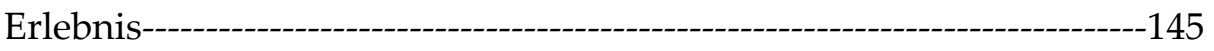

8. Ergebnisse------------------------------------------------------------------------------146

8.1 Homogenität der Gruppen------------------------------------------------------------146

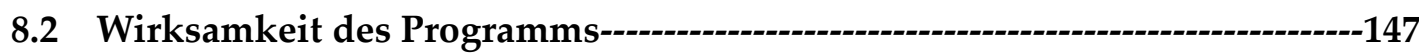

8.2.1 Prä-Post-Vergleich----_-147

8.2.1.1 Prä-Post-Vergleich der depressiven Symptome---------------147

8.2.1.2 Prä-Post-Vergleich allgemeiner psychischer Beschwerden-149

8.2.1.3 Prä-Post-Vergleich des psychischen Wohlbefindens----------151 


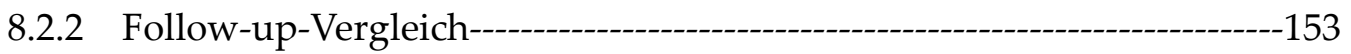

8.2.2.1 Follow-up-Vergleich der depressiven Symptome-------------154

8.2.2.2 Follow-up-Vergleich allgemeiner psychischer Beschwerden --$-156$

8.2.2.3 Follow-up-Vergleich des psychischen Wohlbefindens--------158

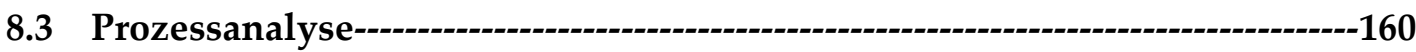

8.3.1 Entwicklung der „depressiven Symptome“ bei der Präventionsgruppe-$-160$

8.3.2 Das hilfreichste Erlebnis während des Programmsverlaufs--------------162

8.3.2.1 Schritt 1: Die Sortierung der gesamten beschriebenen Erlebnisse $-162$

8.3.2.2 Schritt 2: Häufigkeit des hilfreichsten Erlebnisses in dem

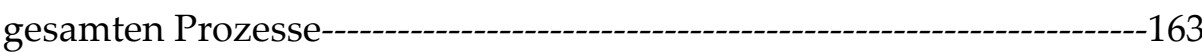

8.3.2.3 Schritt 3: Die herausgefundenen phasenabhängigen bzw. -unabhängigen Hilfsfaktoren----------------------------------------166

8.3.2.4 Schritt 4: Kategorisierung des hilfreichsten Erlebnisses------167

8.4 Annahme des Programms: Rückmeldung der Teilnehmerinnen-------------170

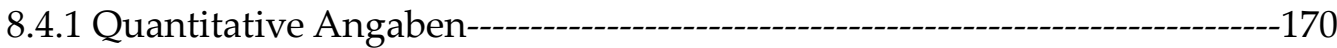

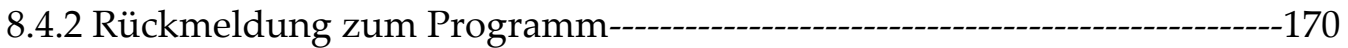

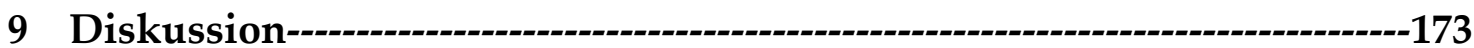

9.1 Wirksamkeit des Programms-------------------------------------------------------173

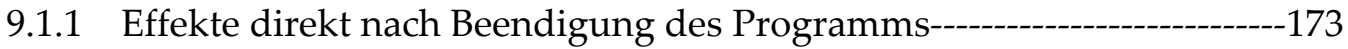

9.1.2 Langfristige Effekte nach drei und sechs Monaten-----------------------176

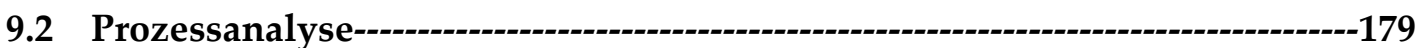

9.2.1 Zusammenhang der Verlaufsentwicklung von Depression und

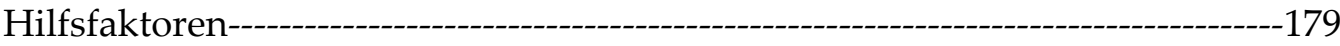

9.2.2 Zusammenhang zwischen den Arbeitsprinzipien und dem hilfreichsten

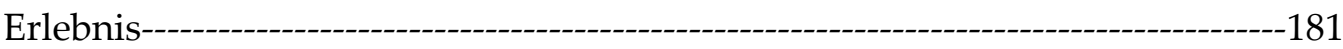

9.3 Entwicklung und Anwendung eines Präventionsprogramms bei psychischen Problemen für die Gesundheitsförderung in dem Kontext „Schule“-

9.4. Relevanz der Prävention von Depression bei weiblichen Jugendlichen---187

9.5. Einschränkungen der Untersuchung und Forschungsausblick---------------189 


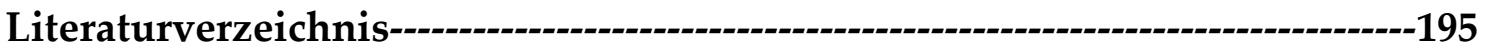

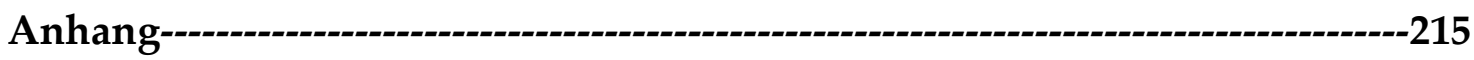

A. Darstellung der im Program gestalteten Produkte von Teilnehmerinnen------216

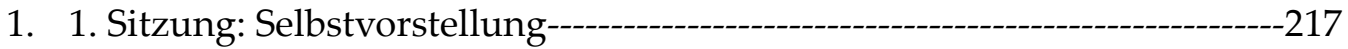

2. 2. Sitzung: Familie in Tieren--------------------------------------------218

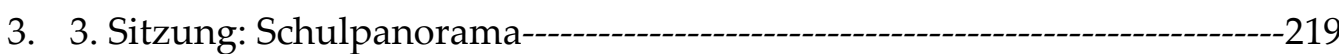

4. 4. Sitzung: Negative Gefühle zum Selbst------------------------------------220

5. 6. Sitzung: Unterbrochene Gefühle zu einer bestimmten Person------------221

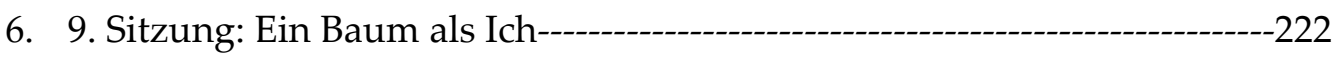

B. Sitzungen des Programms im Überblick--

C. Prä-Post-Follow-up-Vergleich der Mittelwert und Standardabweichung der

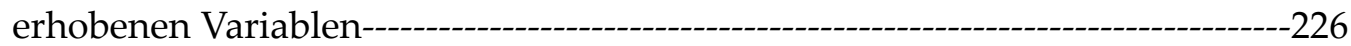




\section{Abbildungsverzeichnis}

Abbildung 8.1: Prä-Post-Vergleich der Mittelwerte des Skalenwerts BDI in beiden Gruppen $-149$

Abbildung 8.2: Prä-Post-Vergleich der Mittelwerte der Depressivität der SCL-90-R in

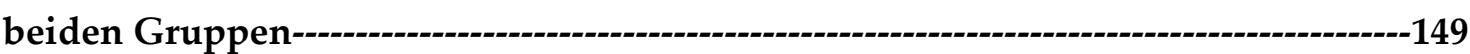

Abbildung 8.3: Prä-Post-Vergleich der Mittelwerte des Skalenwerts GSI(SCL-90-R) in beiden Gruppen 151

Abbildung 8.4: Prä-Post-Vergleich der Mittelwerte des Skalenwerts PWB in beiden

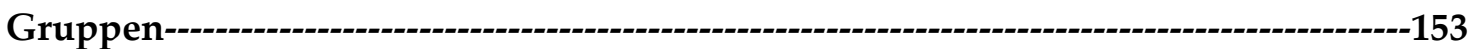

Abbildung 8.5: Vergleich der Verlaufsentwicklung BDI in beiden Gruppen---------156 Abbildung 8.6: Vergleich der Verlaufsentwicklung Depressivität der SCL-90-R in

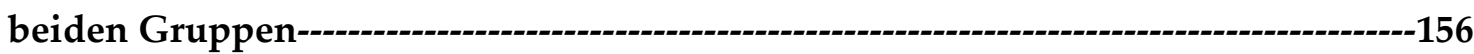

Abbildung 8.7: Vergleich der Verlaufsentwicklung allgemeiner psychischer Beschwerden in beiden Gruppen-----------------------------------------------------158

Abbildung 8.8: Vergleich der Verlaufsentwicklung psychoschen Wohlbefinden in

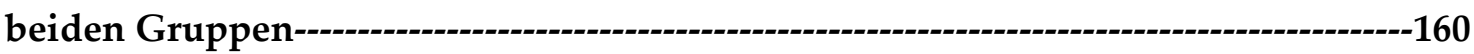

Abbildung 8.9: Verlaufsentwicklung der "depressiven Symptome“ bei der

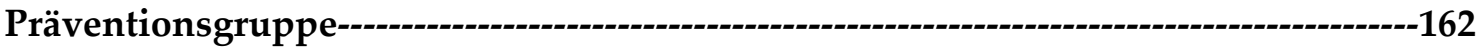

Abbildung 8.10: Entwicklungder hilfreichen Erlebnisse über alle Sitzungen-----170 


\section{Tabellenverzeichnis}

Tabelle 7.1: Teilnahmewünsche an demProgramm----------------------------------------129

Tabelle 7.2: Sozialdemographische Daten der Teilnehmerinnen----------------------131

Tabelle 7.3: Beziehung zu ihrenEltern der Teilnehmerinnen----------------------------133

Tabelle 7.4: Der stärkste Stressfaktor der Teilnehmerinnen------------------------------134

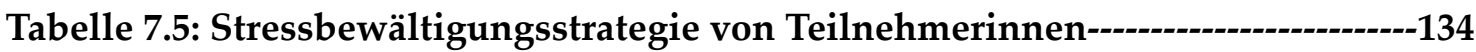

Tabelle 7.6: Grundstimmung der Teilnehmerinnen in der Schule---------------------135

Tabelle 7.7: Symptom-Checkliste 90-R Skalen und globale Kennwerte in der

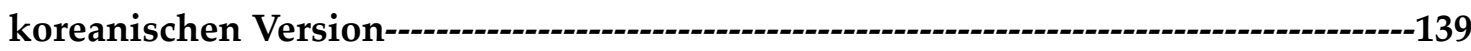

Tabelle 7.8: Mittelwert und Standardabweichung der SCL-90-R in der Jugendstichprobe der Normalbevölkerung in Korea------------------------------------140

Tabelle 7.9: Definitionen theoriegeleiteter Dimensionen des psychischen

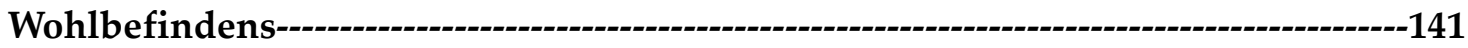

Tabelle 7.10: Messzeitpunkte-----------------------------------------------------------------144

Tabelle 8.1: Mittelwerte zum ersten Messpunkte beider Gruppe (Versuchs- und

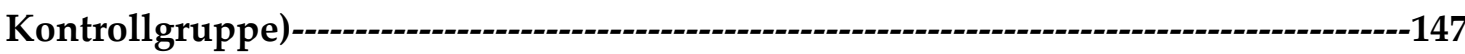

Tabelle 8.2: Prä-Post-Vergleich der depressiven Symptome----------------------------148

Tabelle 8.3: Prä-Post-Vergleich allgemeiner psychischer Beschwerden-----------------150

Tabelle 8.4: Prä-Post-Vergleich der Mittelwerte und Standardabweichungen der Subskalen der SCL-R-90 in beiden Gruppen--------------------------------------------152

Tabelle 8.5: Prä-Post-Vergleich des psychischen Wohlbefindens---------------------153

Tabelle 8.6: Prä-Post-Vergleich der Mittelwerte und Sdandardabweichungen der Subskalen des psychischen Wohlbefindens in beiden Gruppen---------------------154 Tabelle 8.7: Varianzanalyse mit Messwiederholung über vier Zeitpunkte für

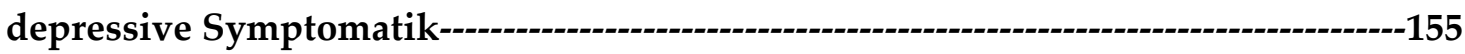

Tabelle 8.8: Varianzanalyse mit Messwiederholung über vier Zeitpunkte für

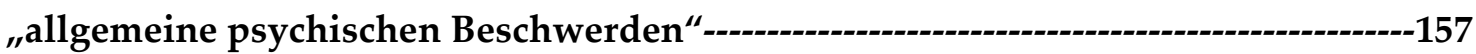

Tabelle 8.9: Follow-Up-Vergleich der Subskalen allgemeiner psychischen Beschwerden in beiden Gruppen--------------------------------------------------------159

Tabelle 8.10: Varianzanalyse mit Messwiederholung über vier Zeitpunkte für

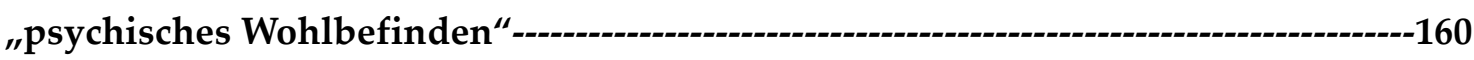

Tabelle 8.11: Follow-Up-Vergleich der Subskalen des psychischen Wohlbefindens in

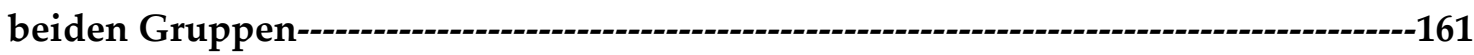

Tabelle 8.12: Velaufsentwicklung der "depressiven Symptome" bei der

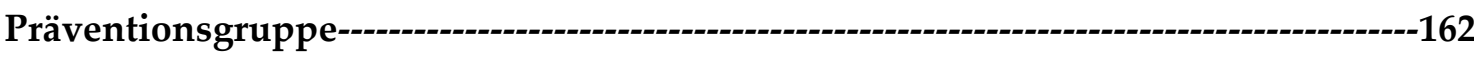


Tabelle 8.13: Die Typen des hilfreichste Erlebnisses----------------------------------------164

Tabelle 8.14: Häufigkeit des hilfreichsten Erlebnisses im gesamten Prozess---------165

Tabelle 8.15: Häufigkeit der Erlebnisse in drei Therapiephasen-----------------------166

Tabell 8.16: Die phasenabhängigen bzw. -unabhängigen Hilfsfaktoren---------------167

Tabelle 8.17: Kategorisierung der Erlebnisse-----------------------------------------------169

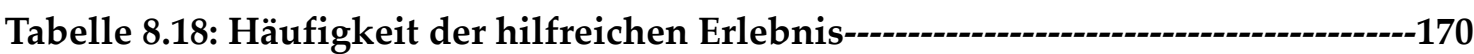

Tabelle 8.19: Rückmeldungen über die Veränderungen während oder nach der

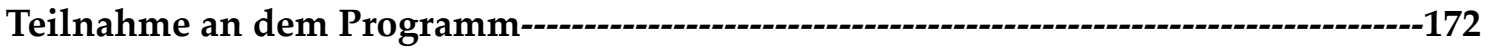




$\begin{array}{ll}\text { Abkürzungsverzeichnis } \\ \text { BDI } & \text { Beck's Depression Inventory } \\ \text { df } & \text { Zahl der Freiheitsgrade (degrees of freedom) } \\ \text { DSM-IV } & \text { Diagnostical and Statistical Manual of Mental Disorders 4. Eds. (APA) } \\ \text { EFT } & \text { Emotion-focused Therapy: Process-experiential therapy (PEF) } \\ \text { GPD-J } & \text { Gestaltprogramm zur Prävention von Depression Jugendlicher } \\ \text { ICD-10 } & \text { International Classification of Diseases } \\ \text { M } & \text { Arithmetisches Mittel } \\ \text { N } & \text { Zahl der Versuchpersonen } \\ \text { PWB } & \text { Inventar zur Erfassung psychischer Wohlbefinden (Psychological Well- } \\ & \text { Being Scale) } \\ \text { SCL-R } & \text { Symptom-Check-List-90-R } \\ \text { SD } & \text { Standardabweichung } \\ \text { T } & \text { Prüfgröße des t-Tests }\end{array}$

\section{Übersetzung von englischen Fachbegriffen}

1. Awareness: Gewahrsein

2. unfinished business: unterbrochene Gefühle oder Prozesse

3. Emotion-focused Therapy: Emotionsfokussierte Therapie

4. Symptom-Check-List: Allgemeine psychische Beschwerde/Belastung

5. Psychological Well-being: Psychisches Wohlbefinden

sonstige Anmerkung: Der einfachen Lesbarkeit halber wird in dieser Arbeit die maskuline Schreibweise als geschlechtsneutrale Form verwendet. 


\section{Danksagung}

Als mir Perls in einem Buch zum ersten mal begegnete, war seine Stimme für mich wie ein sanftes Wiegenlied, damit sich das Kind, das wegen der Beschäftigung mit dem In-der-Welt-Sein ermüdet war, endlich in Ruhe erholen kann. Danach war sie für mich eine Provokationsrede, die mir vorschrieb, in der Welt mein zu Hause zu suchen. Seit dem Anfang meines gestalttherapeutischen Lehrjahres denke ich immer wieder über einen Satz nach, der Heidegger zugeschrieben wird. Der Sazt ist für mich bei der Beschäftigung mit hilfsbedürftigen Menschen ein Leitfaden. „Es ist schwer, verstanden zu werden und doch eine Beleidigung, verstanden zu werden“.

Was seit meiner Begegnung mit der Gestalttherapie in mir entstanden ist, waren Worte, die wie Vögel aus mir entströmten und in dieser Arbeit ihren Ort fanden. Dabei halfen mir viele Freunde mit ihrer großartigen und wohlwollenden Unterstützung. Ihre Namen möchte ich hier gern erwähnen: die Betreuerin dieser Arbeit, Prof. Dr. Christina Krause, die ich „meine deutsche Mutter" nennen möchte, von der ich wissenschaftlich sowie persönlich viel gelernt habe, Prof. Dr. Olaf-Axel Burow, der sich für mich als unterstützender Betreuer erwiesen hat, Prof. Dr. Jungkyu Kim, der mir einen Weg zur Gestalttherapeutin gezeigt hat und somit als mein erster Lehrer für mich immer bedeutsam sein wird, meine Lehrtherapeutin Dr. Lotte Hartmann -Kottek, die mich gelehrt hat, wie man als Gestalttherapeut wissenschaftlich und praktisch lebendig und fruchtbar sein kann. Dank auch an den Stipendiumsfonds Kim’s Scholastika, von dem ich für ein Jahr ein Stipendium für meine Promotion bekomme und Frau Ursula Ziegler-Reinhardt und Hannelore Heuer, die sich um sprachliche Korrektur meiner Arbeit bemüht haben.

Für meine Freunde gilt auch ein herzliches Dankeschön: Dr. Kwansoo Bak, für dessen wissenschaftlichen Rat und wohlwollende Unterstützung, Dr. Ludwig Möller, der mich in meiner schwersten Zeit begleitet hat, Kordula Thöne, Maya Barbara Albrecht, Andreas Blümel und Tilman Hüneke, deren Korrekturarbeit und deren herzliche Freudschaft.

Besten Dank auch den beteiligten Schülerinnen am Projekt sowie dem Schulleiter und den drei Lehrerinnen. Ohne sie wäre die Studie nicht möglich gewesen. 
Meinem Bruder Jongkeun möchte ich für seine vielfache und unvergessliche Unterstützung danken. Für meine Eltern, die mich eine so wunderschöne Welt erleben lassen, gibt es leider nicht genügend und passende Sätze zum Dank. Meinem Vater, der vor kurzer Zeit auf die andere Seite gegangen ist, widme ich diese Arbeit. 



\section{Meinem Vater}

\section{Sun Young Lee}

(05. Feb. 1942 - 29. Aug. 2007)

gewidmet 


\begin{abstract}
In this research, the attempt was made to develop a program for indicative prevention of depression among adolescents, based on Gestalt group therapy with creative media, and to examine its effect in case of depressions, as well as supporting experiences during the process of therapy.

The program GPD-J (Gestalt therapeutical indicative prevention of depression in adolescents) was, on Gestalt therapeutical and emotion-focussed foundations, combined with the methodical implementation of creative media. The treatment program against depression was taken from manual EFT. It consists of 10 therapeutical sessions, in which the participants, for 2 hours each, spend time with creative media. For the implementation of the program, 44 female school students ranging between 16 and 18 years of age, who had volunteered, were selected for subclinical depressive symptomatic under the criteria DSM- IV and after the grades of BDI (Beck's Depression Inventory). The students were randomly allocated to one of two groups, test group and control group. In respect to depressive symptomatic, both groups were homogenous. In order to establish the effectivity of the program GPD-J, both test aqnd control group were tested psychologically before intervention, then 3 months after and again 6 months after.

Summarizing, in regard to the effectivity of the program GPD-J with the support of psychometric tests, a significant reduction of depressive symptoms and psychical complaints, and a significant increase of psychical well-being could be observed. Based on the results it can be stated that the test group, during intervention, made clear progress, and that, by comparison to control group, clear improvements in the areas of depression, depressivity, psychical complaints and psychical well-being were achieved. Further, a significant improvement of test group at the time of both follow-up tests was observed in all areas beforementioned, while no improvement was observed in control group. The long-term effects after three and six months proved to be coherently statistically significant. The strongest impact in test group was to be observed immediately after intervention. During the program the strongest effect appeared in test group in the fifth session, when the BDI values sank dramatically.

Further, during therapy it was examined, which experience during the program was useful for participants. The experiences selected by the participants were analyzed qualitatively to establish the effective factors. During process analysis, four separate auxiliary factors were established. Three phase-dependent auxiliary factors - during
\end{abstract}


the initial phase, in the middle phase and in the final phase - and one phaseindependent factor, which proved supporting throughout the program. The experiences were categorized after qualitative analysis, by "expression" (category I), "contact with others" (category II), "problem" (category III) and "selfperception" (category IV). The auxiliary experiences were sorted by category, and the frequency of their occurrence analyzed. Results show that "self-percetion" (category IV) was regarded most useful by the participants, followed in that order by "expression" (category I), "contact with others" (category II) and "problem" (category III). Category I ("expression") was most mentioned in sessions 1 and 5. Category II ("contact with others") was mentioned in 11 sessions, but most frequently in session 5.

Category III ("problem") was most frequently mentioned in session 7. Category IV, "self-perception" was mentioned increasingly from the seventh session onward, most frequently in the seventh session.

On the whole, in this research project the proof was made that the program GPD-J as a means of indicative prevention of depression among adolscents can be effective. 



\section{Einleitung}

\section{Einleitung}

In der vorliegenden Dissertation wird eine Programm zur indikativen Prävention von Depression bei Jugendlichen entwickelt und die Wirksamkeit dieses Programms überprüft. Das Behandlungskonzept baut auf den gestalttherapeutischen und emotionsfokussierten therapeutischen Grundlagen auf und ist mit den Methoden von kreativen Medien kombiniert. Darüber hinaus wird der Therapieprozess analysiert.

Im Jugendalter steigt die Prävalenz der depressiven Störungen sprunghaft an (vgl. Burke et al. 1990). Die an der Depression erkrankten Jugendlichen weisen hoch komorbide psychische Störungen (vgl. Birmaher et al. 1996), psychosoziale Beeinträchtigungen in verschiedenen Funktionsbereichen (vgl. Compas et al. 1997) und mit hoher Anzahl Suizidplanungen und/oder -versuche (vgl. Bronisch 2003; Essau et al. 1998) auf. In vielen Fällen verläuft die Depression bei Jugendlichen chronisch (vgl. Essau \& Petermann 1997). Empirische Evidenz besteht dahingehend, dass die jugendlichen Depressiven in bis zu 50\% der Fälle einem erhöhten Risiko für zukünftige Major Depression im frühen Erwachsenenalter ausgesetzt waren (vgl. Rao et al. 1999). Dennoch sind drei Viertel der depressiven Jugendlichen ohne angemessene Behandlungen geblieben (vgl. Rohde, Lewinsohn \& Seeley 1991). Aber die Entwicklung wirksamer präventiver Interventionsprogramme zur Vorbeugung depressiver Störungen im Jugendalter ist noch ungenügend.

Obwohl sich gestalttherapeutische Interventionen für die Behandlung von Depressionen bei Erwachsenen als sehr effektiv zeigten (vgl. Greenberg \& Watson 1998; Missirlian et al. 2005; Pos et al. 2003; Rosner 1999; Rude \& Bates 2005; Tyson \& Lange 1987), sind sie bei Jugendlichen bis jetzt kaum angewandt worden. Sie könnten auch zur Entwicklung effektiver Behandlungs- bzw. Präventionsprogramme von depressiven Jugendlichen beitragen.

Bei der Entwicklung von Behandlungs- bzw. Präventionsprogrammen wurde das Jugendalter trotz hoher Prävalenz der depressiven Störungen zwischen Kindes- und Erwachsenalter ziemlich vernachlässigt. Deswegen sollte vor der Entwicklung eines Programms zunächst das Alter der Zielpopulation berücksichtigt werden, da es nicht gerechtfertigt erscheint, therapeutische Interventionen von Erwachsenen auf Kinder und Jugendliche unverändert $\mathrm{zu}$ übertragen (vgl. Grawe et al. 1994). Das breite Problemspektrum bei Jugendlichen zeugt als das „schwierigste Teilgebiet der 


\section{Einleitung}

Psychotherapie“ (Märtens \& Petzold 1995) von einer gravierenden Unkenntnis von Jugendlichen und macht die Handlungserfordernisse für die Situation von Jugendlichen deutlich. Dies fordert von Therapeuten und Beratern, dass sie mit Jugendlichen auf verschiedenen Altersniveaus mit je spezifischem Entwicklungsstand und verschiedenem Schweregrad der Probleme umgehen können müssen. Offensichtlich gilt dies auch bei der Entwicklung der Interventions- bzw. Präventionsprogrammen für depressive Jugendliche.

In der vorliegenden Arbeit werden zunächst die spezifischen Merkmale von Depression bei Jugendlichen dargestellt (Kap. 1). Dann werden zum Verstehen von Depression in der gestalttherapeutischen Perspektive die theoretischen Grundlagen der Behandlung von Depression untersucht, wobei insbesondere das Behandlungsmanual von der emotionsfokussierten Therapie (EFT) für den Aufbau der Entwicklung eines Präventionsprogramms einer Behandlung von Depression bei Jugendlichen berücksichtigt wird (Kap. 2). Auf den Grundlagen der gestalttherapeutischen bzw. emotionsfokussierten therapeutischen Behandlungen wird die Effektivität der Behandlung von Depression, besonders mit Rücksicht auf Jugendliche, in der Kombination mit dem methodischen Einsatz von kreativen Medien untersucht (Kap. 3 und 4) und daraus ein Präventionsprogramm für depressive Jugendliche zusammengestellt (Kap. 6).

In der Arbeit soll die Wirksamkeit dieses Programms untersucht werden. Es soll aber auch der Therapieprozess analysiert werden,um herauszufinden, was in Verlaufe der Therapie besonders hilfreich für Jugendliche war (Kap. 5). Das Programm GPD-J (Gestattherapeutisches Präventionsprogramm von Depression bei Jugendlichen) basiert auf der Gruppenarbeit mit den „Prozess- und themenbezogenen“ Prinzipien. Es besteht aus 10 therapeutischen Sitzungen, in denen sich die Teilnehmer jeweils mit einem Thema beschäftigen. Das Programm hat drei detaillierte Arbeitsschritte, nämlich „erlebnisorientierte Exploration“, „aktiver Ausdruck“ und „Integration“, die aufgrund der vier Wirkfaktoren nach Grawe (1995, 1997b, Grawe et al., 1994) für Arbeitsrichtlinien konzipiert wurden. Das Programm wurde während des regulären Schulbetriebs an einem Gymnasium für Mädchen in Korea erprobt. Zur Feststellung etwaiger Veränderungen wurden die weiblichen Jugendlichen im Alter zwischen 16 und 18 mit Selbstbeurteilungsfragebögen zur Erfassung der „depressiven Symptomatik", der "allgemeinen Symptombelastung“ und des "psychischen Wohlbefinden“" prä-, post und zweimal Follow-up (nach drei bzw. sechs Monaten nach Beendigung der Intervention) getestet. Sie wurden dazu in jeweils zwei Versuchs- und 


\section{Einleitung}

Kontrollgruppen eingeteilt. Darüber hinaus wurde das hilfreichste Erlebnis, über das die Teilnehmer selber nach jeder Sitzung als am meisten hilfreich benannt haben, während des Programmverlaufs in der Versuchsgruppe für Prozessanalyse untersucht (Kap. 7).

Die Wirksamkeit des Programms wird anhand statistischer Auswertungen der psychologischen Tests überprüft. Um den Prozess des Programms zu analysieren, wird das hilfreichste Erlebnis zunächst qualitativ untersucht, dann mit quantitativen Methoden analysiert, um die Wirkfaktoren herauszufinden (Kap.8). Anschließend werden die Ergebnisse, die Einschränkungen der Untersuchung und ein perspektivischer Forschungsausblick diskutiert und zusammengefasst (Kap. 9 und 10).

Ein Programm zur gestalttherapeutischen und emotionsfokussierten Behandlung von depressiven Jugendlichen soll mit kreativen Medien konzipiert und erprobt werden. Das ist eine neue Herausforderung. Ein generalisierender Anspruch auf Wirksamkeit soll durch diese Arbeit nicht erhoben werden. Stattdessen untersucht die vorliegende Studie, in welchem Maße das Programm bei einer Gruppe von Jugendlichen mit subklinischen depressiven Symptomen symptommindernd wirken kann und wie das Programm gestaltet werden muss, damit Hilfsfaktoren in der Arbeit mit Jugendlichen gefunden werden können. 


\section{I . Teil : Theorie}

\section{Depression bei Jugendlichen}

\subsection{Symptomatik}

Als Symptom kann Depression infolge von Enttäuschungen oder Verlusten oder aber auch ohne spezifischen Anlass auftreten. Die Definition einer depressiven Störung im Jugendalter ist schwierig zu erstellen, da sich Depressionen bei Jugendlichen meistens nicht offen, sondern maskiert durch Störungen des Sozialverhaltens, Hyperaktivität, Lernstörungen und somatische Symptome u.a.m. manifestieren. Die Schwierigkeit eine Definition zu erstellen besteht darin, dass sie nicht klar erklären könnte, „wie eine zugrundeliegende Depression mit einer Vielzahl von Diagnosen verknüpft sein kann und wie derartige Störungen jeweils mit und ohne Depression differenziert werden“" (Steinhausen 1993 S. 151).

Die Klassifikation der affektiven und depressiven Störungen gilt für alle Altersgruppen und berücksichtigt mit Ausnahme der Störung des Sozialverhaltens mit depressiver Störung keine für Kinder und Jugendliche spezifischen Kategorien. Die Klassifikation der affektiven und depressiven Störungen gemäß ICD-10 ist folgendes: manische Episode (F 30), bipolare affektive Störung (F 31), depressive Episode (F 32), rezidivierende depressive Störungen (F 33), Zyklothymia ( F 34.0), Dysthymia (F 34.1), kurze depressive Reaktion (Anpassungsstörung) (F 43.20), längere depressive Reaktion (Anpassungsstörung) (F 43.21), Angst und depressive Reaktion (Anpassungsstörung) (F 43.22) und Störung des Sozialverhaltens mit depressiver Störung (F 92.0).

Nach DSM-IV treten mindestens fünf der folgenden Beschwerden für mindestens zwei Wochen bei einer klinisch relevanten Depression (Major Depression) auf, wobei mindestens eine der zwei ersten Beschwerden vorhanden sein muss:

1. Depressive Verstimmung an fast allen Tagen, für die meiste Zeit des Tages, vom Betroffenen selbst berichtet (z.B. fühlt sich traurig oder leer) oder von anderen beobachtet (z.B. erscheint den Tränen nahe).

2. Deutlich vermindertes Interesse oder Freude an allen oder fast allen Aktivitäten, an fast allen Tagen, für die meiste Zeit des Tages (entweder nach subjektivem Ermessen oder von anderen beobachtet). 


\section{Depression bei Jugendlichen}

3. Deutlicher Gewichtsverlust ohne Diät oder Gewichtszunahme (mehr als 5\% des Körpergewichts in einem Monat); oder verminderter oder gesteigerter Appetit an fast allen Tagen.

4. Schlaflosigkeit oder vermehrter Schlaf an fast allen Tagen.

5. Psychomotorische Unruhe oder Verlangsamung an fast allen Tagen (durch andere beobachtbar, nicht nur das subjektive Gefühl von Rastlosigkeit oder Verlangsamung).

6. Müdigkeit oder Energieverlust an fast allen Tagen.

7. Gefühle von Wertlosigkeit oder übermäßige oder unangemessene Schuldgefühle (die auch wahnhaftes Ausmaß annehmen können) an fast allen Tagen (nicht nur Selbstvorwürfe oder Schuldgefühle wegen des Krankseins).

8. Verminderte Fähigkeit zu denken oder sich zu konzentrieren oder verringerte Entscheidungsfähigkeit an fast allen Tagen (entweder nach subjektivem Ermessen oder von anderen beobachtet).

9. Wiederkehrende Gedanken an Tod (nicht nur Angst vor dem Sterben), wiederkehrende Suizidvorstellungen ohne genauen Plan, tatsächlicher Suizidversuch oder genaue Planung eines Suizids.

$\mathrm{Ab}$ der Adoleszenz verbindet sich die Depression mit oft übersteigerten und verzerrten Gefühlen der Sinnlosigkeit, des Versagens und der Schuld (Steinhausen 1993). Die typischen Symptome im Erwachsenenalter - wie Grübeln, Suizidimpulse und Minderwertigkeitsgefühle - prägen das Bild der Depression auch im Jugendalter. Dabei treten die Hauptsymptome - depressive Stimmung, Interesseverlust, Freudlosigkeit, Antriebsmangel, erhöhte Ermüdbarkeit - und zusätzliche Symptome wie verminderte Konzentration und Aufmerksamkeit, vermindertes Selbstwertgefühl und Selbstvertrauen, Gefühle von Schuld und Wertlosigkeit, Suizidgedanken oder -versuchen, Schlafstörungen und Appetitstörungen und Gewichtsveränderungen auf.

\subsection{Merkmale der Depression im Jugendalter}

\subsubsection{Prävalenz und Verlauf der Depression im Jugendalter}

\subsubsection{Auftreten und Häufigkeit}

Die niedergedrückte oder depressive Stimmung trifft jeden Menschen häufig, aber kann schnell vorübergehen. Im Gegensatz zum normalen Phänomen einer 


\section{Depression bei Jugendlichen}

niedergeschlagenen Stimmung ist die klinische Depression durch die stabile Dauer der Symptome und durch Beeinträchtigung der Funktionstüchtigkeit der Betroffenen gekennzeichnet. Depressive Störungen stellen eine Störungsform mit unterschiedlichen, in vielen Fällen lang anhaltenden negativen Auswirkungen auf die persönliche und psychosoziale Entwicklung der Betroffenen dar (Rohde et al. 1994; Klein, Lewinsohn \& Seeley 1997; Geller et al. 2001, Hautzinger \& Petermann 2003). Es ist für depressive Störungen charakteristisch, dass sich über die letzten Jahrzehnte das Alter bei Erstauftreten signifikant vorverlagert hat und die Auftretensrate im Jugendalter sich stark vergrößert (Hautzinger \& Petermann 2003).

Zahlreiche Studien mit Kindern und Jugendlichen berichten die Prävalenzraten unterschiedlich. Eine gründliche Prüfung dieser Studien für die Unterschiede der Ergebnisse lässt eine Reihe von Gründen vermuten, z.B. verschiedenartige Techniken der Erhebung, unterschiedliche Informationsquellen, der Gebrauch unterschiedlicher Diagnosekriterien (Essau \& Petermann 2000, S. 296). Dennoch berichten die Autoren übereinstimmend, dass die Depressionsraten mit dem Alter zunehmen (Essau 2002, Essau, Conradt \& Petermann 2000, Essau \& Petermann 1997, Kashani \& Carlson 1987, Rutter 1986; Wittchen et al. 1998). Während die Depressionsrate bei Kindern unter 2\% (Essau \& Petermann 1997), bei Vorschulkindern unter 1\% (Kashani \& Carlson 1987) liegt und bei Kindern im Schulalter bei etwa 2\% (Anderson et al. 1987) anzunehmen ist, ist sie im Jugendalter aber deutlich ansteigend. Eine Studie zeigt, dass depressive Störungen am häufigsten zwischen dem 15. und 19. Lebensjahr beginnen und ihre Prävalenz ab dem 14. Lebensjahr sprunghaft ansteigt (Burke et al., 1990). In der Regel erkranken die Kinder noch früher an Depression, wenn die Eltern, insbesondere Mütter depressiv sind (Kurstjens \& Wolke 2001).

Über Prävalenz der Depression berichten die Autoren (Essau et al. 2000), dass 21.5\% bei einer Stichprobe von 12- bis 17-Jährigen auf die Diagnose der Major Depressive Disorders nach DSM-IV entfielen. Die Isle of Wright-Studie über die Depressionsrate bei zehnjährigen Jugendlichen durch wiederholte Befragung im Abstand von vier Jahren fand, dass die Depressionen nach vier Jahren um das Zehnfache zugenommen hatten. In einer anderen Studie lag die Depressionsprävalenz bei 14- bis 24-jährigen Jugendlichen insgesamt bei 16,9\%. Die Lebensprävalenz der Major Depression betrug $18,4 \%$ bei 14 - bis 18-Jährigen (Lewinsohn et al. 1993) oder 14\% aller Jugendlichen (vgl. Essau \& Petermann 2000). Ungefähr 17,9\% aller Jugendlichen berichten, schon einmal in ihrem Leben an einer depressiven Störung erkrankt zu sein (Essau et al. 1998a, b, zit. nach Essau \& Petermann 2000). Das Durchschnittsalter, in dem depressive Störungen erstmals auftraten, lag nach einer Studie (Lewinsohn et al. 1993) bei 14,3 Jahren. 


\section{Depression bei Jugendlichen}

\subsubsection{Stabilität und Rückfall}

Die meisten Erwachsenen mit Depressionen berichten über einen frühen Beginn der Störungen, vorwiegend vor dem 20. Lebensjahr (Wittchen et al. 1992). In vielen Fällen verläuft die Depression bei Jugendlichen chronisch (Essau \& Petermann 1997) und stabil (Groen \& Petermann 2002). Bei der Dauer depressiver Episoden zeigen sich große Unterschiede. Die durchschnittliche Dauer der depressiven Episode bei Jugendlichen beträgt zwischen einigen Monaten und einem dreiviertel Jahr (Groen \& Petermann 2002). Aus der Erhebung bei erwachsenen Depressiven berichtet man, dass bis zu 40\% das Ersterkrankungsalter als in der Kindheit und Jugend liegend angeben wurde (Geller \& Luby 1997). Empirische Evidenz besteht dahingehend, dass die jugendlichen Depressiven in bis zu 50\% der Fälle einem erhöhten Risiko für zukünftige Major Depression im frühen Erwachsenenalter ausgesetzt waren (Rao et al. 1999). Aus einer klinischen Studie entwickelten 30\% mit Dysthymer Störung nach 24 Monaten eine Major Depression, 50\% nach 40 Monaten (Kovacs et al. 1984b). Groen und Petermann (2002) betonen, dass sich bei Kindern und Jugendlichen mit Major Depression hohe Remissionsraten von bis zu über 90\% nach einem bis zwei Jahren zeigen, das Risiko eines Rückfalls oder einer wiederholten Depressionsdiagnose groß ist und zwischen etwa 25\% nach einem Jahr und etwa 75\% nach fünf Jahren liegt. Es wird von einigen Autoren festgestellt, dass Kinder und Jugendliche mit depressiven Störungen auch als Erwachsene ein signifikant erhöhtes Risiko für ein erneutes Auftreten depressiver Episoden haben (Birmaher et al. 1996; Lewinsohn et al. 1999).

\subsubsection{Psychosoziale Beeinträchtigung}

Depressive Jugendliche zeigen im weiteren Leben stärkere psychosoziale Beeinträchtigungen in verschiedenen Funktions- und Lebensbereichen als Gleichaltrige ohne psychische Störungen. Unter anderem lassen sich häufiger soziale und zwischenmenschliche Probleme, schulische oder berufliche Schwierigkeiten, eine höhere Inanspruchnahme therapeutischer Hilfen, Konsumabhängigkeit, Selbstmordversuche und vollendete Selbstmorde sowie mehr allgemeine gesundheitliche Probleme feststellen (vgl. Groen \& Petermann 2002, S. 64; Shaw 1988). Verschiedene Studien legen nahe, dass depressive Symptome möglicherweise ihren Niederschlag in der eingeschränkten Fähigkeit finden, angemessen mit Anforderungen im Unterricht umzugehen, sowie mit der Unfähigkeit, angemessen mit Gleichaltrigen zu interagieren (Shaw 1988). Die depressiven Jugendlichen haben signifikant häufiger 


\section{Depression bei Jugendlichen}

von Schwierigkeiten mit ihren Lehrern und Klassenkameraden (Birmaher et al. 1996, Groen 2002), schlechten Schulleistungen, delinquentem Verhalten (Fleming et al. 1993), Kompetenz- und Konfliktlösungsdefiziten (Rudolph et al. 1994) berichtet.

Jugendliche setzen sich ebenso wie Erwachsene mit dem Thema Tod und Suizid auseinander. Die hohe Anzahl von 17,9\% der Jugendlichen mit Major Depression gaben an, einen konkreten Suizidplan gehabt zu haben, und 9,7\% unternahmen sogar einen Suizidversuch (Essau, et al. 1998a, b). Weissmann et al. (1999) fanden, dass das Risiko für einen ersten Selbstmordversuch in der Gruppe der Depressiven fünffach erhöht war. Insgesamt war bei den depressiven Jugendlichen das lebenszeitbezogene, relative Risiko für einen Selbstmordversuch 14 Mal höher als bei Jugendlichen ohne psychische Störung (zit. nach Groen \& Petermann 2002, S. 68). In der Republik Korea, wo die empirische Studie für die vorliegende Arbeit durchgeführt wurde, wurde im Rahmen des Gesundheitssurveys Depression als Ursache von etwa 80\% der Selbstmorde geschätzt. Selbstmord ist in Korea die erste Todesursache bei Jugendlichen im Alter 15 bis 24 Jahren.

Nach epidemologischen Studien zeigen depressive Störungen im Vergleich zu anderen Störungen das höchste Ausmaß an Komorbidität. z. B. Depressionen mit Verhaltensstörungen (Kovacs et al. 1989), Angststörungen (Kovacs et al. 1989), Aufmerksamkeits-Hyperaktivitätsstörungen (Bukstein et al. 1992), Essstörungen bei Mädchen (Herpetz-Dahlmann 1993), Aggressivität (Reicher 1999, Weiss \& Carton 1994) und Substanzmissbrauch (Bukstein et al. 1992; Lewinsohn et al. 1998, Essau et al. 1998). Der Prozentsatz depressiver Jugendlicher zeigt, dass etwa $40 \%$ bis $70 \%$ aller depressiv erkrankten Jugendlichen komorbide psychische Störungen (Birmaher et al. 1996) oder 42,8\% der Jugendlichen mit Major Depression in der Lebenszeitdiagnose eine weitere psychische Störung (Lewinsohn et al. 1998a) aufwiesen. Jugendliche erleben depressive Gefühle als eine Mischung von Traurigkeit und Ärger, wobei Ärger gegen sich oder gegen andere gerichtet sein kann (Renouf \& Harter 1990). Essau (2000) zeigte, dass das häufigste Komorbiditätsmuster bei Mädchen das von Angst und Depression und bei den Jungen von Depression und Störungen durch Substanzkonsum ist.

\subsubsection{Risikofaktoren für depressive Störungen im Jugendalter}

Es liegt zum heutigen Zeitpunk keine einheitliche, empirisch gestützte Theorie zur Entwicklung und Aufrechterhaltung depressiver Störungen im Kindes- und Jugendalter vor (Groen \& Petermann 2002). Die Forschung an depressiven Kindern und Jugendlichen zeigt eine erhöhte Wahrscheinlichkeit, dass Verlustereignisse eines 


\section{Depression bei Jugendlichen}

Elternteils durch Tod, Scheidung oder Trennung am häufigsten das Risiko für das Auftreten depressiver Störungen vergrößern (Compas et al. 1994). Allerdings stehen dabei alltägliche, chronische Belastungen als kritische Lebensereignisse in engerem Zusammenhang mit der Depressivität (Seiffe-Krenke 1993). Aber hier soll noch betont werden, dass weder ein bestimmter Stressor ganz spezifisch das Auftreten depressiver Störungen bewirkt, noch bei allen exponierten Personen den gleichen schädigenden Effekt haben muss. Auf Stressoren und Belastungen reagieren Menschen vielmehr ganz unterschiedlich, und teilweise wiederum in Abhängigkeit von bestimmten Personen- und Umfeldmerkmalen, d.h. der Einfluss von Stressoren wird als komplexe Interaktion gesehen (Rossmann 2001). Für die Entstehung depressiver Störungen im Jugendalter gibt es verschiedene Erklärungsansätze, die je nach kognitiv-behavioralen, interpersonalen und multifaktoriellen bzw. entwicklungspsychopathologischen Perspektiven und solchen von Stress und dessen Bewältigung konzipiert werden. Im Rahmen verschiedener Erklärungsansätze wird das zunehmende Bemühen deutlich, die multifaktorielle, bio-psycho-soziale Verursachung von Depression nachzuzeichnen. Zusätzlich werden in besonderer Weise Entwicklungsaspekte des Jugendalters berücksichtigt (Groen \& Petermann 2002). Hier stellten Wiesner und Reitzle (2001, S. 249) drei allgemein gehaltene Grundannahmen heraus: Erstens werden depressive Störungen im Jugendalter durch das Zusammenspiel mehrerer Faktoren verursacht. Zweitens können je nach Alter und Entwicklungsstand unterschiedliche Faktoren für die Entstehung depressiver Störungen verantwortlich sein. Und drittens ist nicht auszuschließen, dass die verschiedenen depressiven Störungsbilder auch unterschiedliche ätiologische Modelle erfordern. Heute besteht die Aufgabe darin, Antworten auf die Fragen zu finden, welche Faktoren die weite Verbreitung sowie stetige Zunahme und systematische Vorverlagerung des Alters bei Erstauftreten der Depression bedingen.

Dabei wird aus entwicklungspsychopathologischer Perspektive bei Depression im Jugendalter angenommen, dass eine depressive Störung in verschiedenen Entwicklungsphasen und in unterschiedlichen Fällen auf ganz unterschiedlichen Risiken und Entwicklungswegen beruhen kann (Hautzinger \& Petermann, 2003). Dabei kommen u.a. nicht nur alle Vulnerabilitäten, Prädispositionen oder Umfeldbedingungen, sondern auch Resilienz und protektive Faktoren in Untersuchungsfragen zur Sprache, wobei der Blick auf Depression nicht nur problemorientert, sondern gleichzeitig schutzorientiert werden muss. 


\section{Depression bei Jugendlichen}

\subsection{Schwierigkeiten früher Intervention bei Depression von Jugendlichen}

Bis jetzt ist nach den Ergebnissen zahlreicher Studien bekannt, dass die Prävalenz der depressiven Störungen im Jugendalter erhöht wird. Trotz der Zunahme der Depression im Jugendalter werden drei Viertel der depressiven Jugendlichen aus verschiedenen Gründen nicht oder nicht angemessen behandelt (Rohde, Lewinsohn \& Seeley 1991). Hier besteht die Aufgabe darin, zu erklären, warum die Depressionen bei Jugendlichen nicht angemessen behandelt wurden. Wird davon ausgegangen, dass die Depressionsprävalenz in der Pubertät stark ansteigt, ist es nicht so einfach für Betroffene und Bezugspersonen einzuschätzen, was genau die depressive Verstimmung verursacht, weil verschiedene Veränderungen in dieser Phase geschehen und sie für alle Betroffenen neu sind. Im Jugendalter befinden sich die Menschen in einer besonderen Situation, in der das Einsetzen umwälzender körperlicher und emotionaler Veränderungen und das Hinterfragen von Werten, Überzeugungen und Identität stattfinden. Jugendliche fühlen sich selbst fremd und verwirrt.

Die Forschungsergebnisse aus der Jugendpsychologie zeigen, dass es viele Jugendliche gibt, die diesen schwierigen Lebensabschnitt ohne Probleme durchlaufen (Powers et al. 1989; Golombek 1990). Die Mehrheit der jungen Menschen bewältigt diesen Lebensabschnitt ohne größere psychische Störungen, indem sie eine sichere persönliche Identität sowie positive Sozialkontakte entwickeln.

Trotzdem ist die Zunahme der Depressionen im Jugendalter dramatisch hoch. Verschiedene Faktoren dieser Entwicklungsphase gelten als besonderes Risiko für die Entstehung einer Depression. Wenn sich Jugendliche in dieser Phase aus eigener Kraft mit dieser Entwicklungsaufgabe nicht genug auseinandersetzen können, dauert die depressive Verstimmung, die in diesem Alter durchaus normal ist, länger und die Wahrscheinlichkeit einer psychischen Störung wird erhöht.

Groen und Petermann (2002) vermuten Ursachen für die Zunahme der Depression im Jugendalter im Folgenden: biologische und psychosoziale Auswirkungen der Pubertät, allgemeine Entwicklungsaufgabe der Jugendzeit, Rollendifferenzierung, Zunahme schulischer Anforderungen etc. (vgl. S. 102). Jedoch ist bei der Bewertung der Annahmen zum erhöhten Depressionsrisiko zu berücksichtigen, dass die einzelnen genannten Faktoren nicht per se als gefährdend oder risikoerhöhend einzustufen sind (Groen \& Petermann 2002). Deshalb ist es schwierig festzustellen, ob ein Jugendlicher unter einer Depression leidet.

Ein anderer Grund, warum depressive Jugendliche ohne angemessene Behandlungen verbleiben, besteht in dem internalisierten Charakter der depressiven Symptombilder. 


\section{Depression bei Jugendlichen}

Depressive sind oft still und zurückgezogen trotz ihrer somatischen Probleme und Komorbiditäten. Hängt der Energie- und Freudeverlust als ein Symptomcharakter der Depression mit dem Jugendalter zusammen, wird er eher vernachlässigt als externalisierte Symptome wie Hyperaktivität und Substanzkonsum. Somatische Probleme z.B. Bauch- und Kopfschmerzen zeigen sich nicht nur bei depressiven Jugendlichen, sondern auch häufig bei allen Jugendlichen. Es gibt eine Tendenz von Eltern und Gesellschaft, dass sie die gedämpften oder apathischen Jugendlichen eher ertragen als Jugendliche, die offenkundig problematisch sind, wobei die Jugendlichen dann ohne Hilfe unter der Depression leiden. Wenn die Betroffenen im Extremfall durch Selbstmord ihr Leiden gezeigt haben, ist es zu spät.

\subsection{Präventionsforschung über Depression im Jugendalter}

Im Folgenden sollen die Formen und Ziele der Prävention erklärt werden, so dass die Probleme der Präventionsprogramme und Bedingungen bei der Entwicklung eines Programms diskutiert werden können. Davon hängt die Effektivität der Präventionen ab.

Präventive Interventionen werden unterschieden in drei Formen, nämlich Primär-, Sekundär- und Tertiärprävention (Caplan 1964; Mrazek \& Haggerty 1994). Dieses Klassifikationssystem wird aufgrund weitgefasster und unscharfer Trennung zwischen Prävention und Behandlung als problematisch angesehen. Folglich wurde es durch ein neues System ersetzt: Präventiv-, Behandlungs- und Erhaltungsinterventionen. Präventivinterventionen selbst werden innerhalb dieses Klassifikationssystems in drei Unterformen eingeteilt: universelle, selektive und indikative Präventivinterventionen (vgl. Wiesner \& Reitzle 2001, S. 249-250).

Werden die drei Präventivinterventionen im Bereich der Depressionsprävention im Jugendalter angewandt, lassen sie sich wie folgt unterscheiden: Universelle Präventivinterventionen richten sich prinzipiell an alle Jugendlichen der gesamten Bevölkerungsgruppe oder Subpopulationen und basieren auf der Annahme, dass die präventive Maßnahme für alle Populationsmitglieder, unabhängig von eventuell vorhandenen individuellen Risiko- oder Schutzfaktoren, die gewünschten Effekte zeitigt. Bei Zielgruppen, in denen sich Jugendliche mit statistisch erhöhtem Risiko befinden, eine Depression zu entwickeln, handelt es sich um eine selektive Intervention. Diese Jugendlichen sind anhand vorliegender biologischer, psychologischer oder sozialer Risikofaktoren identifiziert, z.B. solche mit einem affektiv erkrankten Elternteil (vgl. Junge et al. 2002; Pössel \& Hautzinger 2003; Wiesner 


\section{Depression bei Jugendlichen}

\& Reitzele 2001). Sind es Jugendliche bereits mit Symptomen oder frühen Vorboten der Depressionen oder psychischen Störungen, deren Kriterien für die Diagnose jedoch zum Zeitpunkt der Intervention nicht erfüllt sind (z.B. subklinische Depressionsscores), gehören sie zu der Zielgruppe mit indikativer Präventivintervention. Ziele werden hier zusätzlich auf die Senkung der Symptomdauer und Verhinderung der Eskalation zu schwerwiegenderen Symptombildern ausgerichtet (vgl. Wiesner \& Reitzele 2001). Die Unterscheidung zwischen indikativer und selektiver Präventivintervention scheint in manchen Studien aufgrund unterschiedlicher Diagnosekriterien und verschiedenartiger Risikofaktoren unklar. Selektive Präventionsmaßnahmen werden deswegen oft mit indikativer Prävention ohne Unterscheidung durchgeführt (Clarke et al. 1995, Groen \& Petermann 2002).

Präventionsziele von psychischen Störungen werden in ein distales und ein proximales Ziel unterschieden. Distales Ziel ist die Prävention einer Zielstörung, z.B. Erfassung der Depressivität der teilnehmenden Jugendlichen. Das ist oft nicht die Senkung der Anzahl neu auftretender depressiven Störungen, sondern die Reduktion bzw. Konstanthaltung depressiver Symptome bei unauffälligen Jugendlichen bzw. bei Jugendlichen mit subklinischer Depression. Bei einem proximalen Ziel handelt es sich um die positive Beeinflussung von Prädiktoren oder Risikofaktoren für die Zielstörung. Das kann je nach theoretischem Schwerpunkt der Autoren wechseln, z.B. Entwicklung der interpersonalen Beziehungsfähigkeit oder Senkung dysfunktionaler Gedanken. Die Beeinflussung der proximalen Faktoren wird in der Regel ebenfalls als Programmeffekt gewertet. Sie stellt eine notwendige Bedingung für die Erreichung der Programmziele dar (vgl. Manz, Junge \& Margraf 2001, S. 169; Pössel \& Hautzinger 2003, S. 155).

\subsection{Probleme bei der Programmentwicklung für Depressionsprävention}

Es ist dringend erforderlich, wirksame Präventionsprogramme für Depressionen bei Jugendlichen angesichts verschiedener Risikofaktoren $\mathrm{zu}$ entwickeln und ihre Effektivität zu beweisen angesichts unterschiedlicher Schweregrade, Erscheinungsformen depressiver Symptome und altersspezifischer Maßnahmen im jugendlichen Entwicklungsbereich. Vor der Entwicklung angemessener Programme und ihrer Überprüfung der endgültigen Auswirkungen sollte man darauf achten, welche Probleme bei vorhandenen Präventionsprogrammen angegeben wurden. Die Probleme liegen für mich in folgenden Dimensionen.

Erstens gibt es in jeder Form eines Präventionsprogramms Vor- und Nachteile. Im Vergleich aller Formen der Prävention, universal, selektiv und indikativ, sind 


\section{Depression bei Jugendlichen}

verschiedene Punkte zu berücksichtigen. Die vorliegenden universellen Programme wurden während des Schulunterrichts durchgeführt. Thre Effekte sind geringer als bei selektiven und indikativen Formen. Obwohl die universellen Programme eine Vielzahl kleinerer Effekte bei einer größeren Anzahl von Teilnehmern haben, bedeutet das eine große Auswirkung auf die Gesellschaft (vgl. Pössel \& Hautzinger 2003). Es bleibt zu klären, ob Depressionsprävention für alle Jugendlichen notwendig gebraucht wird, wenn die Jugendlichen von selbst depressive Verstimmung gut überwinden, ohne sie als schwierig zu erachten, oder sie überhaupt ganz von depressiven Symptomen verschont bleiben. Sollte trotzdem Prävention erfolgen, muß geklärt werden wann genau sie durchgeführt werden sollte. Es ist bekannt, dass das Auftreten der Depression in der Pubertät stark angestiegen ist, aber es ist bisher unbekannt, wie der Verlauf depressiver Störungen im Jugendalter vor sich geht. Dies ist epidemologisch nicht erforscht.

Bei Rekrutierung eines selektiven oder indikativen Programms ist es fraglich, welche Kriterien die Symptome der Teilnehmer erfüllen sollten, um zur Zielgruppe zu gehören, weil die gegenwärtig vorliegenden diagnostischen Instrumente noch unbefriedigend und wenig entwicklungsspezifisch erforscht sind (Hautzinger \& Petermann 2003). Dazu ist es noch bei selektiver bzw. indikativer Prävention problematisch, dass es eine hohe Drop-Out Rate (Pössel \& Hautzinger 2003), negative Sozialkontakte durch Peers (Schochet et al. 2001) und eine Chronifizierung durch Labeling (Harrington \& Clark 1998, zit. nach Pössel \& Hautzinger 2003) gibt. Das Problem der hohen Drop-Out Rate im Jugendalter gilt nicht nur für die Prävention, sondern auch für allgemein alle psychischen Interventionen bei Kindern und Jugendlichen. Nach einer Studie von Armbruster und Kazdin (1994) brechen etwa 50\% der Kinder und Jugendlichen die Behandlung vorzeitig ab. Diese Tatsache weist bei der Entwicklung und Anwendung eines selektiven oder indikativen Präventionsprogramms darauf hin, dass ein Programm zur Reduzierung der Wahrscheinlichkeit eines Drop-Outs bei Jugendlichen konzipiert werden sollte. Wenn die Jugendlichen zur Risikogruppe von Depression gehören, sollten sie die selektive Prävention möglichst nicht abbrechen. Bei der praktischen Anwendung selektiver bzw. indikativer Prävention können Teilnehmer durch Labeling von Schulkameraden und von sich selbst Nebenwirkung zeigen, so dass sie einerseits von der Peergruppe schwache bzw. negative soziale Kontakte übernehmen und andererseits depressive Störungen chronisch weiter behalten können. All diese Nebenwirkungen spielen zusammen.

Aber solche Probleme werden bei universeller Prävention auch gesehen. Wenn 


\section{Depression bei Jugendlichen}

universelle Prävention für alle Schüler wie üblich im Schulunterricht durchgeführt wird, können die Jugendlichen von den Schulkameraden, die bessere Bewältigungsstrategien haben, lernen (vgl. Pössel \& Hautzinger 2003), während die unmotivierten Jugendlichen relativ inaktiv und hilflos gegenüber der Interventionsmaßnahme werden können. Folglich sind die unmotivierten, inaktiven Jugendlichen, die wahrscheinlich schon mit Risikofaktoren belastet sind oder in den Prozess depressiver Störungen eintreten, in Gefahr, von unangemessener Prävention zusätzlich belastet zu werden, weil sie ohnehin im Interventionsverlauf wie im Unterricht oft passiv oder blockiert sind, ihr internalisiertes Selbst und ihre Gefühle zum Ausdruck zu bringen.

Zweitens beziehen sich die Probleme in Bezug auf das Ziel der Prävention auf die Ergebnisse verschiedener Präventionsprogramme. Thr distales Ziel -im Sinne von Reduktion oder Linderung depressiver Symptome- haben die zahlreichen Programme zur Depressionsprävention im Vergleich zwischen Versuchs- und Kontrollgruppe mit Follow-Up-Erhebung nicht erreicht (Beardslee et al. 1997; Clarke et al. 1993, Study 1, 2; Groen et al. 2003; Kellam et al. 1994; Petersen et al. 1997). Das Problem in Hinsicht auf distale Ziele liegt besonders in universellen Programmen, während die proximalen Ziele entweder erreicht oder nicht erreicht wurden. Als Gründe nennen Groen et al. (2003, S. 171), dass die Studien zur Feststellung der Effekte mit sehr großen Stichprobengruppen realisiert werden müssen, da die Effekte von universellen Präventionsmaßnahmen auf Veränderung des Depressionsniveaus schwer zu bestimmen sind (Pössel \& Hautzinger 2003) und dass sich relevante Auswirkungen von Präventionsmaßnahmen auf die Depressionssymptomatik erst über noch längere Follow-Up-Zeiträume einzustellen scheinen, weil bedeutsame Unterschiede der Depressivität zwischen Versuchs- und Kontrollgruppe bei der Evaluation selektiver Präventionsprogramme erst nach 18 Monaten festgestellt wurden (Gillham \& Revich 1999; Gillham et al. 1995, zit. nach Groen et al. 2003). Groen und seine Mitarbeiter (2003) sagen dazu optimistisch, dass das Programm trotz Nichterreichens des distalen Zieles bei den proximalen Zielen, z.B. kognitive Faktoren, Erfolge erzielt habe und in zukünftigen Studien untersucht werden, ob die Effekte auf proximale Ziele als moderierende Variablen mit späteren Auswirkungen auf Depressivität in Zusammenhang stehen. Hier stellt sich jetzt die dringliche Frage, wozu überhaupt ein Präventionsprogramm gegen Depression dienen soll, wenn ein Programm keinen Interventionseffekt bei Depressionssymptomatik zeigt. Als Hauptziel eines Präventionsprogramms gegen Depression wird gewertet, ob es Effekte zur Linderung oder Reduktion depressiver Symptome hat oder nicht. 


\section{Depression bei Jugendlichen}

Drittens geht es um Probleme hinsichtlich der Interventionsmaßnahmen und ihrer Ausgangsfragen entweder von einem spezifischen Geschehen depressiver Störungen oder von multifaktoriellem Geschehen. Das Präventionsprogramm bezieht sich entweder auf problematisches Verhalten oder auf die Suche nach Resilienz und Ressourcen oder auf beides. Die spezifische Ätiologie von depressiven Störungen wie von anderen psychischen Störungen ist noch weitgehend unbekannt. Alle heutigen ätiologischen Konzepte für depressive Störungen gehen von einem Zusammenwirken unterschiedlicher bio-psycho-sozialer Faktoren aus (Hautzinger \& Petermann, 2003) aus. Hieraus ergibt sich die Notwendigkeit, komplexe, multifaktorielle und schulenübergreifende Interventionsmaßnahmen $\mathrm{zu}$ konzipieren, die eine Fülle potenzieller Risikofaktoren (vgl. Manz, Junge \& Margraf 2001) und Resilienz- und Schutzfaktoren (Dumont \& Provost 1999) gleichzeitig thematisieren.

Bleibt noch zu erklären, wie ein Präventionsprogramm gegen Depression Jugendliche mit bzw. ohne Symptome überhaupt beeinflussen kann. Hinsichtlich direkter Auswirkungen eines Präventionsprogramms sollte überprüft werden, welche unerwartete Wirkung dabei auf die Teilnehmer ausgeübt wird. 


\section{Gestalttherapie und Depression}

\subsection{Zum Verstehen der Depression in gestalttherapeutischer Perspektive}

\subsubsection{Depression und Kontaktunterbrechung}

\subsubsection{Kontakt und seine Funktion}

In der Gestaltherapie ist Kontakt der zentrale Begriff, und wird sowohl als "Prozess" als auch als "Zustand", beispielsweise zwischen Organismus und Umwelt verstanden. Ein Mensch als Organismus befindet sich in jedem Moment mit seiner Umwelt im Kontakt und wird durch Kontakt ernährt. Kontakt ist für das Überleben des Menschen basal und fundamental. Wenn ein Mensch Durst hat, nach Wasser sucht, endlich Wasser trinkt, ist dies ein kontinuierlicher Prozess. Unter dem Begriff Kontakt als „Prozess" versteht man Aktivität, Mobilisierung der Energie des Organismus zur Erfüllung des Willens oder des Wollens. Unter dem Begriff Kontakt als "Zustand" versteht man Zenit, Verbundenheit (Bezogenheit) und Einmaligkeit, sobald ein Mensch sein Wollen zum Stillen des Durstes befriedigt. Jeder Kontakt findet einmalig im „Hier und Jetzt“ also an der Kreuzung des Kontextes und des Kontinuums statt. Kontakt findet immer in der Zwischendimension statt. Jede Information über sich selbst, von der Umwelt und von der Beziehung zwischen dem Organismus und der Umwelt wird durch Kontakt verarbeitet. Es geht auch um die Wahrnehmung von Unterschieden, ferner darum, dass der Organismus die Fähigkeit zur schöpferischen Anpassung verwirklicht, sowie seine Identität und seinen Lebenssinn entfaltet. In diesem Sinne ist Kontakt „Berührung und Abgrenzung“.

Wahrnehmung ist im Kontaktprozess notwendig, um sowohl das Eigene als auch das Fremde zu spüren, um das Dazwischen zu erfahren und um neue Dimensionen zu erkennen. Aus dem Kontakt entstehen Begegnung, die intersubjektives Erfassen ist, Beziehung, die dauerhafte Begegnung ist, und womöglich Bindung. In jedem Kontaktprozess gibt es nicht nur die Chance zur Befriedigung sondern auch die potentielle Gefahr, wobei der Organismus gegenüber Anderen Aggression und Gelegenheit haben muss, sie in dem Kontakt wiederum assimilieren zu können. Die Aggression ist ein fundamentaler Bestandteil der Anpassung, denn ohne Aggressivität kann ein Organismus sich nicht anpassen. Aus diesem Grund zielt Aggression auf eine Teilnahme an der Neugestaltung des Organismus und der Gegenstände seiner 


\section{Gestattherapie und Depression}

Umgebung ab. Neugestaltung des Organismus gilt nicht nur für den Organismus selbst, wobei der Organismus seine Verantwortung für das „In-der-WeltSein" entwickelt. Im menschlichen Wachstumsprozess sind sowohl eine ausreichende Menge als auch eine angemessene Qualität des Kontaktes unerlässlich. Wenn man sich wiederholt oder ausschließlich einer Kontaktstruktur aussetzt, die andere Menschen ungenügend oder schädlich beeinflusst, besteht die Gefahr, ein Muster der Kontaktunterbrechung $\mathrm{zu}$ entwickeln. Kontaktmuster, die der Anpassung und Vermeidung dienen, sind Abwehrmechanismen. Die in einem ambivalenten Kontaktgeschehen blockierte Kraft bleibt als „unerledigte Gestalt“ im Organismus zurück. Sie taucht bei passenden Resonanzen wiederum im Vordergrund auf und hat die Tendenz, geschlossen zu werden.

Alle psychischen Probleme entstehen im Kontakt bzw. an der Kontaktgrenze. Wenn ein Mensch gegenüber seiner Umwelt die verschiedensten störenden Gefühle z. B. Ärger, Groll, Enttäuschung hat, muss er seine Energie auf vielfache Weise ableiten, was die Möglichkeiten einer kontaktreichen Interaktion mit seiner Umwelt verringert. In diesem Prozess entstehen verschiedene Muster, die seinen Lebensstil prägen und als die vom Widerstand bestimmten Interaktionen bezeichnet werden (vgl. Polster \& Polster 1975, S. 77). Das „Kontaktmuster ${ }^{1 "}$ wird in dieser Arbeit als die Form der Kontaktunterbrechung bezeichnet. Der Begriff Kontaktmuster beinhaltet die Struktur der Kontaktfunktionen und wird nach sechs Arten sortiert: Konfluenz, Introjektion, Projektion, Retroflektion, Egotismus ${ }^{2}$ und Deflektion.

Der Kontaktzyklus wird als Welle der Kontaktenergie im Sinne des Kontaktprozesses verstanden. Fritz Perls (1944) nennt den Kontaktzyklus „Stoffwechsel-Zyklus“ mit sechs Phasen: 1.Organismus in Ruhe, 2. Störfaktor, 3. Erschaffung eines Vorstellungsbildes, 4. Antwort, 5. Verminderung der Spannung, 6. Rückkehr zum Gleichgewicht. Perls, Hefferine und Goodman (1951) beschreiben den Kontaktzyklus mit seiner schöpferische Anpassung in vier Phasen: 1. Vorkontakt, 2. Kontaktnehmen, 3. Kontaktvollzug, 4. Nachkontakt. Zinker(1977) und Nevis (1988) aus der Cleveland School nennen den Vorgang „Zyklus des Erlebens" und unterscheiden sechs Phasen: 1.

\footnotetext{
${ }^{1}$ In der vorliegenden Arbeit wird das Kontaktmuster ausgewählt, da es die Art und Weise des Kontakts meint. Kontaktmuster bedeutet hier fixierte Kontaktgewohnheit, beinhaltet Funktion im Kontaktprozesses, ist aber nicht unbedingt Störung. Je nach Kontext und Kontinuum ist die Funktion des Kontaktmusters entscheidend, ob es Störung ist. Manche Autoren benutzen bevorzugt den Begriff „Unterbrechungen des Kontakts“, „um die nicht gewollte Assoziation zu psychiatrisch diagnostizierten Störungen der Persönlichkeit zu vermeiden“ (Blankertz \& Doubrawa 2005 S. 181).

2 Bei Perls gehört Egotismus zum Kontaktmuster, bei Polster \& Polster gehört stattdessen Deflektion.
} 
Empfindung, 2. Gewahrsein, 3. Mobilisierung von Energie, 4. Handlung, 5. Kontakt, 6. Lösung, 7. Abschluss/Rückzug. Ähnliches findet sich bei Clarkson (2004), und sie differenziert seine "Gestalt formation and destruction" in sieben Phasen: 1. Sensation, 2. Awareness, 3.Mobilization, 4. Action, 5. Final Contact, 6. Satisfaction, 7. Withdrawal. Blankertz und Doubrawa (2005) nennen ihn als Gestaltwelle mit sechs Phasen: 1. Vorkontakt 2. Kontakt mit dem Bedürfnis 3. Kontakt mit der Umwelt 4. Aggression 5. Assimilation, 6.Nachkontakt. Hartmann-Kottek (2004) beschreibt den als Wachstumskreis verstandenen Kontaktzyklus in neun Phasen. Sein Phasenmodell hatte bereits Perls (1944) durch analoge Untergliederungen abgestuft.

Die Hauptgedanken des Modells kreisen um den Figur-Hintergrund-Kontrast. Dabei entsteht die Vordergrundfigur durch die energetisierende Aufmerksamkeit ihrer Gestalt. Die Gestaltbildung - folgt einer motivational ausgerichteten, selbstorganisatorischen Verwandlungskette (vgl. Hartmann-Kottek 2004). Der Kontaktzyklus gilt als ein Symbolisches Geschehen für den Prozess der Selbstregulation. Es ist dabei zu berücksichtigen, dass der Kontaktzyklus zwischen den einzelnen Phasen diskontinuierlich verlaufen kann (vgl. Fuhr \& Gremmler-Fuhr 2002), dass die Phasen überlappend sind (Melnick \& Nevis 1997), ferner, dass es unerlässlich ist, dass sich der Organismus in jeder Phase mit dem Kontakt zur jeweiligen Vordergrundfigur mit Erfolg identifiziert. Kann eine Kontaktphase nicht in die nächste Phase übergehen, entsteht eine Kontaktunterbrechung, die „nicht gesund“, vielmehr dysfunktional ist. Das Phasenmodell des Kontaktzyklus kann „als Orientierung und Suchraster, als Ausgangspunkt für die Bildung von Hypothesen zur Erforschung von innerpersönlichen und zwischenmenschlichen Prozessen, als ReflexionsPlanungsinstrument“ (Fuhr \& Gremmler-Fuhr 2002, S.101) sowie als DiagnoseInstrument (Blankertz 2004; Dreitzel 2004; Melnick \& Nevis 1997; Müller 1988, 1999; Swanson \& Lichtenberg 1998) auf vielfache Weise gute Dienste leisten.

In der vorliegenden Arbeit wird das Modell des Kontaktzyklus und der Kontaktmuster als Ausgangspunkt zum "gestalttherapeutischen" Verstehen und für "gestalttherapeutische" Hypothesenbildung zur Erforschung von depressiven Prozessen und als Selbstreflexionsentwurf, durch den die Arbeit für depressive Prozesse als „Voranschreiten von Fokus zu Fokus" und die prozessdiagnostische Konzeption zur Verfügung steht, genutzt. Eine gestaltorientierte Arbeit kann durch gestalttherapeutische Hypothesen bzw. eine Diagnose, der die Gestalttherapeuten wenig Aufmerksamkeit geschenkt oder sie vernachlässigt haben (vgl. Hutterer-Krisch 1999; Melnick \& Nevis 1997; Müller 1999; Swanson \& Lichtenberg1998), ihren eigenen Charakter mit eigenen spezifischen Wirkfaktoren gewinnen und sich widerspiegeln, so 
dass eine Krankheit nicht als eine gültig festgestellte, sondern „eine aktive Konstruktion von Wahrheit (vorläufige Gewissheit), die überhaupt erst therapeutisches Handeln ermöglicht und die durch selbstkritische Reflexion immer wieder hypothetischen Charakter gewinnt" (Müller 1999, S. 649), gesehen werden.

Da die Motive menschlichen Erlebens und Verhaltens viel zu komplex sind und ihre Vielfalt nicht in wenige Kategorien eingeordnet und ihre Eigenarten in nicht bestimmten Entwicklungsphasen wie nach einem historisch-kausal orientierten Determinismus fixiert werden können, erschweren vor allem erkenntnistheoretische Prämissen der Gestalttherapie die Motivation zur Entwicklung einer gestalttherapeutischen Diagnostik (vgl. Müller 1999, S. 648).

Melnick und Nevis (1997) schlagen eine gestalttherapeutische Diagnose für Borderline Persönlichkeitsstörungen, Phobien, hysterische Persönlichkeitsstörungen und PTSD im Bezug auf den Kontaktzyklus und DSM-IV vor. Swanson und Lichtenberg (1998) versuchen zu erklären, wie die gestalttherapeutische Diagnose mit sechs vorgestellten Leitlinien im Kontaktprozess mit Kontaktunterbrechung funktioniert. Kim (2003) erklärt Angststörungen in Bezug auf den Kontaktzyklus und die Kontaktmuster für die gestalttherapeutische Behandlung mit Ergänzung der kognitiv-behavioristischen Therapie und der Meditation. Bertram Müller (1999) stellt ein kategoriales Modell gestalttherapeutischer Diagnostik für hysterische Persönlichkeitsstörung, zwanghafte Persönlichkeitsstörung, die schizophrene Erlebens und Verhaltensstörung und die Borderline-Erlebensstruktur dar, wobei folgende drei Themenbereiche des Selbst im Zusammenhang des Kontaktzyklus und des Kontaktmusters konzipiert wurden: die zeitlichen Aspekte der Figur-Grundbildung des Selbst, die Teilfunktionen des Selbst und die Qualitäten des Selbst.

Dreitzel (2004) erklärt mit dem Entwurf einer gestalttherapeutischen Diagnostik die verschiedenen Störungen auf verschiedenen Ebenen. Ihre Ansätze basieren auf der Fragestellung, wie eine Stšrung gestalttherapeutisch gesehen werden kann. Dadurch regen die Autoren dazu an, dass die Gestalttherapie ihre bisherige Zurückhaltung aufgeben und aktiv an der therapeutischen Praxis teilnehmen möge. Gestalttherapeuten gehen davon aus, dass ein fixiertes Kontaktmuster während der Anpassung des Organismus an eine neue Situation mit dem Ziel der Selbstabwehr und jeweils zur Vermeidung des sich hingebenden Kontakts entwickelt wird, wonach der Organismus trotz immer neuer Situationen typischerweise doch immer wieder mit einem ähnliche Verhaltensmuster reagiert und folglich eineschöpferische Anpassung verhindert. Darüber hinaus entwickeln sich die gestalttherapeutischen Diagnostikansätze als ein Raster aus der Grunddynamik des Kontaktzyklus und ihrer 


\section{Gestattherapie und Depression}

unterschiedlichen Grundstörungen (vgl. Müller 1999).

Die obigen Autoren (Clarkson 2004; Hutterer-Krisch 1999; Kim 2003; Melnick \& Nevis 1997; Müller 1999; Swanson \& Lichtenberg1998), die zur Entwicklung der Diagnostikansätze das Phasenmodell „Kontaktzylus“ verwendet haben, sehen außer Clarkson (2004) und Kim(2003) den gewohnheitsmäßigen Stillstand des Kontaktprozes in einer speziellen Phase als das psychische Problem an. Natürlich kann jede Phase als ein eigenes Phänomen von den anderen Phasen getrennt charakterisiert werden. Aber jede Phase ist dennoch ein Teil des ganzen Prozesses und stellt selbst einen Prozess dar. Das Problem entsteht jeweils durch eine Unterbrechung am Übergang zwischen den einzelnen Phasen des Prozesses.

\subsubsection{Depressive Prozesse und Kontaktunterbrechung}

Es sind zunächst zwei Schritte für die Diagnostik von Bedeutung: die allgemeine klinische Diagnostik und eine schulen- bzw. orientierungsspezifische Diagnostik (Schuch, 2000). Hier werde ich versuchen darzulegen, wie die Depression oder das depressive Syndrom mit der gestalttherapeutischen Perspektive hinsichtlich der Kontaktfunktion in Bezug auf den Kontaktzyklus verstanden werden kann.

\section{Phase 1: Im Übergang von Empfindung zum Gewahrsein}

In dieser Phase der Empfindung wird der Organismus durch den Reiz und/oder das Bedürfnis stimuliert. „Bedürfnis ist Erfahrung von Mangel“ (Dreitzel 1992) aus dem Empfinden eines Mangels entsteht sogleich ein spezifischer Wunsch, die Vorstellung einer Konkretion der Abstraktion, sodass sich nun die Kontaktgrenze von innen nach außen verschiebt. Ein Gewahrsein dessen, was an der Kontaktgrenze vor sich geht, meint ein bewusstes Erleben und "reflexive Sinnlichkeit" (Dreitzel 1992), da Gewahrsein „ein Prozess des In-aufmerksamem-Kontakt-Seins mit dem wichtigsten Ereignis im Organismus/Umwelt-Feld auf der Basis voll entfalteter sensomotorischer, emotionaler, kognitiver und energetischer Kräfte“ (Yontef 1979, S.27) ist. Das Gewahrsein erfüllt zwei Funktionen im Kontaktprozess: Es ermöglicht Problemlösungen und Frustrationstoleranz (Dreitzel 1992, S. 64). Das bedeutet, es wird dann versucht, Gewahrsein herabzusetzen, wenn ein Organismus Angst hat, im Kontaktprozess Frustration zu erleben.

Beispiel: Obgleich ein Organismus selbst seine körperliche Präsenz empfunden hat, könnte er es verhindern und verleugnen, dass diese Empfindung in den Vordergrund kommt, sodass er sein Bedürfnis nicht in den Kontaktprozess eindringen lässt. Es bleibt 
folglich im Organismus in einer verdrängten Form zurück. Die Angst vor Frustration oder vor „blockierten Gefühlen“ (unfinished business) verfremdet die ursprüngliche Wahrnehmung, anstatt sie zu identifizieren. Bei Depressiven wird zum Beispiel aufgestaute, unerledigte Aggressivität vom Organismus kaum oder nicht zugelassen, sodass entsprechende psychische und körperliche Empfindungen im Hintergrund vernachlässigt werden. An diesem Übergang nehmen Depressive aggressiv Getöntes nicht wahr, sowohl wenn es von ihnen selbst stammt, wie wenn es von draußen stammt. Sie behindern das Hervortreten einer Vordergrund-Figur, indem sie sich selbst oder anderen keine Aufmerksamkeit schenken, oder, indem sie absichtlich Aufmerksamkeit von sich auf andere umleiten, was in gewisser Weise der Bedürfnisbefriedigung dient. Obwohl sie ganz traurig aussehen, sagen sie immer: „Es geht mir ganz normal" oder "gut". Leute, die chronische Erfahrungen mit „unterbrochenden Gefühlen oder Prozessen“ (unfinished business) haben, haben Angst davor, ihre Frustration deutlich wahrzunehmen, da die Gefühle für sie chronisch bedrohliche Erinnerungen hervorrufen.

Darüber hinaus können sie nicht in weitere Kontaktprozesse zur Befriedigung einsteigen sondern unterdrücken Gefühle, Erregungen und Bedürfnisse. Diese verschwinden nicht, sondern bleiben im Organismus. Es handelt sich meist um solche, die von denen des Gegenübers abweichen und unterschwellig meist eine autonomaggressive Färbung haben. Sie verursachen bei den Depressiven, die zum Somatisieren neigen, psychosomatische Probleme. In dieser Phase empfinden sie die Signale aus ihrem Körper als fremd, oft sogar als angstauslösend. Diese Besonderheit an diesem Übergang muss die therapeutische Arbeit für Depressive besonders berücksichtigen und es gilt achtsam zu unterstützen, wie die Betroffenen ihre Stimuli, Erregungen und Bedürfnisse wahrnehmen kšnnen. Die depressiven Menschen projizieren oft ihre eigenen Stimuli und Erregungen nach draußen, als ob diese nicht ihre eigenen wären. Sie reduzieren mit Deflektion ihr eigenes Gewahrsein zum Kontakt, damit gar nichts passieren kann. Hier steht die Empfindung selbst nicht im Vordergrund der Arbeit, sondern sie hilft den Personen, das Empfundene als etwas Eigenes wahrzunehmen, damit es im Kontaktprozess weiterhin zugelassen werden kann.

\section{Phase 2: Im Übergang vom Gewahrsein zur Mobilisierung der Energie}

Hier wird die Kontaktenergie des Organismus zur Mobilisierung blockiert, nachdem er sein eigenes Bedürfnis wahrgenommen hat. Die nicht zugelassene Energie verbleibt in seinem Körper, indem seine Organe oder seine Muskeln aktiviert und angespannt werden. Wenn diese Energie chronisch blockiert und zu einem Selbstläufer-Programm 
wird, ergeben sich spezifische, psychosomatische Fehlinnervationsmuster und Beschwerden. In dieser Phase erlebt der Organismus dann Angst. Auf diese Weise erklären Kim, (2003) und Melnick \& Nevis (1997) Psychoneurose und Angststörung. Obwohl der Organismus genug darüber „weiß“, was er für eine angemessene Handlung zur Befriedigung tun muss, kann er beim Depressiven seine Energie zur Handlung nicht weiter zur Verfügung stellen, weil er innerlich mit seiner Energie nicht in Kontakt kommt. So scheitert er an dieser Stelle. Die Energie wird in den meisten Fällen aus Angst vor heftiger Erregung oder vor starken Gefühlen blockiert. Die unbewusste Blockierung tritt physiologisch häufig von einer flachen Atmung begleitet auf mit der Folge, dass die Versorgung des Körpers mit Sauerstoff reduziert wird und das Energieniveau abfällt. (Zinker 1982). Dann kommt der Organismus in einen Teufelkreis: die Energie fließt in die falsche Richtung, die Versorgung des Körpers wird reduziert, so dass sich der Mensch erschöpft erlebt, ohne, dass die ursprüngliche Erregung abgeklungen ist. Ein solcher Prozess wird bei Depressiven oft beobachtet. Erschöpfung, Müdigkeit, Lustlosigkeit und beeinträchtigte Körperempfindungen sind ihr typischer Zustand.

In dieser Phase wird gegenüber der Mobilisierung der Energie bei Depressiven ganz allgemein eine starke Retroflexion entgegengesetzt. Sie wenden sich gegen sich selbst, anstatt sich gegen die zu wenden, die ihr Missfallen erregen. Sie hören auf, sich um sich selbst zu kümmern, beispielsweise um ihr Essen oder ihr Aussehen. Sie retroflektieren gegenüber Menschen, die sie lieben oder die sie sich gewogen erhalten möchten, das heißt, sie haben Angst davor, problematische Gefühle z.B. Ärger, Unbehagen oder Unzufriedenheit zum Ausdruck zu bringen. Stattdessen lenken sie den Ärger und die Kritik auf sich selbst, so dass sie unter dieser Selbstkritik selbst leiden. Dabei verlieren sie weitgehend ihre Lebendigkeit, bekommen ein Gefühl der Leere, werden missmutig, machen sich Selbstvorwürfe und haben Insuffizienzgefühle. Das sind Merkmale der pathologischen, depressiven Reaktion nach Mentzos (1984). Zu Unbehagen, Ärger oder Unzufriedenheit kommt noch der Verlust ihrer vitalen Lebensenergie hinzu.

Wenn die Reflexion zu einer chronischen Distanzierung zwischen den entgegengesetzten Energien innerhalb des Menschen wird, dann geht der natürliche Rhythmus zwischen Selbstbeobachtung und Spontaneität verloren, und der Verlust dieses Rhythmus spaltet den Menschen in sich gegenseitig behindernde Kräfte (Polster \& Polster 1975 S. 89). Wenn ein Kind in der Kindheit von den Eltern negative Erfahrungen zum Ausdruck seiner Gefühle beigebracht bekommen und die introjizierten Bilder davon in sich gespeichert hat, etwa, dass es nicht wertvoll ist, wird 
sein Prozess zur Mobilisierung der Energie blockiert (vgl. Clarkson 2004). Da diese Menschen Angst vor ihrer eigenen Erregung (Excitement) haben, oder davor, Kontakte zuzulassen, versuchen sie ihre Energie zu verleugnen, sodass sie sich zum Beispiel ins Bett legen oder kaum etwas tun, um ihre Energie nicht spüren.

Innerhalb des Gestaltprozesses bei Depressiven ist das Überbrücken von Blockierung zwischen Gewahrsein und Mobilisierung der Energie entscheidend. In diesem Prozess wird in der Gestaltarbeit so gearbeitet, dass man mit seiner Energie in Kontakt kommen soll, beispielsweise in einem kontrollierten Experiment, in dem man seine körperlichen Empfindungen und seine Gefühle zum konkreten physischen Ausdruck bringt. Die Verleugnung des eigenen Wollens, der eigenen Energie und der eigenen Gefühle in der fortgeschrittenen Phase der Kontaktnahme wird erlebt als starke Schuldgefühle, die von Depressiven in einem erheblichen Ausmaß beschrieben werden. Diese Schuldgefühle sind die andere Seite des Wollens: sie entsprechen dem Hemmungserleben des Wunsches, etwas für sich selbst zu wollen. In der Gestaltarbeit werden deswegen zunächst die Willensaspekte beachtet, die fordernd gegen sich selbst gerichtet sind und dabei Schuldgefühle machen können. Dann lässt man die projizierten Persönlichkeitsanteile rückintegrieren und schließlich schrittweise die Verantwortung für die eigenen Gefühle sowie für die Gefühlsäußerungen übernehmen. (vgl. Müller 1999, S. 668).

\section{Phase 3: Im Übergang von Mobilisierung zur Handlung, von der Handlung zum Kontakt}

In dieser Phase versteht man die Handlung mit dem Begriff „Aggression“, den Fritz Perls in einem positiven Sinne verwendet, nämlich in der Funktion der aktiven Gestaltung und Veränderung der gegebenen Situation. Im Blockierungsfall: der Organismus ist bei dieser Form der Blockierung unfähig, an dem Prozess zur Gestaltung und Veränderung mit seinen Impulsen aktiv teilzunehmen ${ }^{3}$ und nicht imstande, seine Energie in die richtige Richtung, die er gern möchte, zu lenken. Mobilisierte Energien und Gefühle gegenüber anderen dürfen oft nicht ausgedrückt werden. Ein Teil davon wird oft projiziert. Projektionen und andere Abwehrmaßnahmen schützen den Organismus und helfen ihm dabei, die negativen Gefühle einigermaßen zu kontrollieren, die, wenn sie erst einmal ausgedrückt worden sind, für ihn oder für andere schädigende oder unethische Folgen haben

\footnotetext{
${ }^{3}$ Das nennt Dreitzel als "Initiative des Ausschreitens und Zugreifens", die ein Element der Aggression als Handlungsmodus ist (1992, S. 66).
} 


\section{Gestattherapie und Depression}

könnten ${ }^{4}$. Als chronisches Wiederholungsmuster bekommt diese Form rasch Krankheitswert. Wenn man dem Projizieren widersteht, kann man in den Schatten der „idealisierenden Selbstenttäuschungen und Rationalisierungen“ geraten. (Zinker 1982) Als Alternative der Projektion kann man die Energie gegen sich selbst wenden. Spontane Reaktionen werden dabei gehemmt, sodass als somatisches Korrelat der Retroflexion häufig Muskelspannungen entstehen. Oft werden vor dem Auftreten der Retroflexion Aggressionsverbote introjiziert (vgl. Hutterer-Krisch 1999). Im schlimmsten Fall wenden sich Depressive in mörderischem Zorn in ihrer Retroflektion gegen sich selbst. Depressive haben die Tendenz ihre Energie nicht nach draußen gehen zu lassen sondern in sich hinein zu drängen. „Im Gegensatz zum Konfluenten kann der Retroflektor die Grenze zwischen sich und der Umwelt ziehen" (Hutterer-Krisch 1999, S.31). Sie identifizieren nicht ihre Aggression zur Gestalt auf ihre Bedürfnisse, die normalerweise nach außen gegen ein bestimmtes Objekt gewendet sind, sondern gegen sich als Gegenstand der Aggression und haben folglich Selbstanklagen, Schuldgefühle, Suizidalität ect. ,JJe stärker die Selbstbestrafung, desto kräftiger die daraus resultierende Wut und die Aggressionsphantasien, die ebenfalls zur Bewusstheit nicht durchdringen“, da die entstandenen Aggressionen nicht ausgelebt und erledigt werden sondern in der Phantasieebene bleiben (vgl. Amendt-Lyon 1999, S. 153).

Oft sind Depressive mit jenem Überfordernden, missbilligenden und Überbeanspruchenden Elternteil identifiziert, der sein Kind wegen seiner hohen Erwartung hasst, so dass sie chronisch in der Situation sind, Frustrationsangst vor den Ergebnissen genauer gesagt vor dem Misslingen haben. Sie sind in der Lage, weder das eigene Ziel zur Handlung zu entwickeln noch Verantwortung für die eigene zur Handlung zu übernehmen. Hier entstehen Konflikte und Polaritäten ${ }^{5}$ Es ist in der Gestaltarbeit unerlässlich, dass die Depressiven nicht zu einem Handeln gedrängt werden, sondern dass der Therapeut den Depressiven ermöglicht, zunächst in einem ganz geringen Ausmaß der Energie Ausdruck zu geben, die er in sich spürt (vgl. Zinker 1982), damit sie die Fähigkeit haben, das eine vom anderen zu unterscheiden. Sie können dann mit den Experimenten ihr Ziel, für das ihre Energie verfügbar ist, realisieren, um dann endlich auf angemessene Weise ihre Energie zur Handlung bringen.

\footnotetext{
${ }^{4}$ Die Entstehung und Funktion der Wut als Notwehrreaktion und als Quelle der Gewalt beschreibt P.H. Dreitzel in seinem Buch (1992, S. 68ff.).

${ }^{5}$ Fritz Perls nennt Depression als Topdog-Underdog-Konflikt. Dieser Konflikt ist ein Polaritätskonflikt, der nicht unbedingt nur diese Phase beeinflusst, sondern jede und späternoch näher ausgeführt wird.
} 


\section{Phase 4: Im Übergang von Kontakt zu Rückzug, von Rückzug zur Empfindung}

In der Phase Kontaktvollzug erlebt man „den freischwebenden Zustand des vollen Kontakts“ (Dreitzel 1992, S. 71) zur hächsten Entfaltung des Selbst im Kontaktprozess. Die Figur der Bedürfnisse, die den Kontakt ausmacht, fließt mit der mobilisierten Energie des Organismus in die Berührung, wobei der Organismus und die Umwelt zur Integration und $\mathrm{zu}$ einer einzigen Erfahrungseinheit verschmolzen werden. Hier entfaltet sich das Selbst als "System6" (Goodman 1988) und als „Prozess" (Dreitzel 1992) mit jedem Bedürfnis, und mit jeder Befriedigung verschwindet es wieder. Der Organismus genießt den Prozess der Sättigung und Befriedigung. Wenn er ihn wirklich empfunden und erlebt hat, hält er sich von dem Kontaktprozess zurück, lässt ihn ausklingen und wartet dann darauf, dass Neues in ihm auftaucht.

Wenn man den Kontakt in dieser Phase nicht vollkommen empfindet, kann man den Kontaktprozess weder genießen noch abschließen. Der Depressive klammert sich an das, was er von dem vollen Kontakt in seiner Phantasie erwartet hat, aber er empfindet oder erlebt nicht den tatsächlich entstandenen Kontakt. Beim Rückzug fühlt er nicht Freude und Zufriedenheit. Es kommt nicht zur Assimilation des Kontaktes mit der Umwelt, sondern es entsteht Enttäuschung oder Trauer. Dann wünscht sich der depressive Mensch die Wiederholung des Kontakts, anstatt sich auf Neues einzulassen. Wenn es eine Synergie zwischen der Erfahrung des Organismus und seiner Kapazität zur Beschäftigung mit der Erfahrung gibt, dann zielt hier der Kontaktvollzug auf einen Rückzug ab, sozusagen auf eine „Demobilisierung“ (Melnick \& Nevis 1997).

Wenn der Organismus in der Lage ist, sich von der Erfahrung zu lösen und sie -symbolisch ausgedrückt- übermäßig und verzehrend zu kauen und zu verdauen, dann kann er mit der Einleitung und der Verwendung der emotionalen Energie den Kontaktprozess „Vollzug-Rückzug-Empfindung“ durchgängig erleben ohne zu unterbrechen. Wenn dieses nicht getan wird, dann wird die neue Figur des Bedürfnisses nicht richtig integriert und unaufhörlich verzerrt, und es geht das Gleichgewicht zu dem gegenwärtigen und zukünftigen Kontakt verloren (vgl. Melnick \& Nevis 1997, S. 102). Deswegen ist die Unterbrechung dieses Prozesses die „Rhythmusstörung“ (Zinker 1982).

Solche beeinträchtigenden Unterbrechungen finden sich oft bei Menschen, die unter

\footnotetext{
${ }^{6}$ Goodman (1988) nennt das Selbst ,als das System der ständig neuen Kontakte“. Das Selbst ist die Kontaktgrenze in Tätigkeit; diese Tätigkeit besteht im Erschaffen von Figuren und Hintergründen. Es ist nur ein kleiner Faktor in der gesamten Organismus-Umwelt-Interaktion des Findens und Schaffens der Bedeutungen, durch die wir wachsen. Deswegen ist das Selbst also nichts Substantielles oder Statisches, sondern ein Prozess (Dreitzel 1992, S. 41).
} 
traumatischen Lebensereignissen leiden oder die sich im Trauerprozess befinden. Depressive sind in dieser Phase unfähig, adäquat zu unterscheiden, was sie von der Welt annehmen und was sie zurückweisen sollen, weil ihre Fähigkeit zur Schaffung der Ich-Umwelt-Grenze eingeschränkt und beeinträchtigt ist, so dass ihre Selbstachtung und ihr Selbstwertgefühl verloren gehen, währenddessen Selbstvorwürfe und Selbstbestrafungen erheblich vermehrt werden. Sie wissen nicht, wie sie den Kontakt dosieren sollen, ob sie genug Stimulation gehabt haben, wann sie den Kontakt loslassen möchten, ob der Kontakt angenehm für sie ist oder nicht und der Kontakt abgebrochen werden sollte. Bei Depressiven entsteht insbesonders ein langdauernder Rückzug, der die folgenden Phasen des Kontaktprozesses, der der Empfindung, des Gewahrseins und der Mobilisierung weitgehend unmöglich macht. Sie sind nicht bereit oder unfähig, für differenzierte Empfindungen künftig offen zu bleiben.

Dreitzel (2004 Kommentar zu Schaubild 10) beschreibt den depressiven Prozess besonders mit Kontaktmustern. Er meint, dass im depressiven Prozess drei wichtige Grundmuster des Kontakts stattfinden: Projektion, Retroflektion und Introflektion. Im depressiven Prozess nimmt man mehr wahr mit Introjektion und Projektion als Grunderfahrung. Man macht keine Unterschiede, sondern achtet nur darauf, ob man scheitert. Man fühlt sich eben dann in der Vorausannahme bestätigt, und erlebt alles immer gleich vertraut negativ. Deshalb sagen die depressiven Menschen; „Ich bin nichts wert", „Ich bin schuld“. Dreitzel meint, dass der Therapeut aufgrund des Hangs zu Introjektionen bei Depressiven vor allem auf die Neigung achten muss, dass jener alles zu schlucken bereit ist, was der Therapeut sagt, um eventuell alles brav zu tun, was der Therapeut vorschlägt. Die depressiven Menschen sagen bei Schwierigkeiten; „Es hat ohnehin keinen Sinn“, „Schon wieder sieht mich niemand“. Er findet bei Depression auch Anzeichen von Retroflektion z.B. im Bereich von Motorik und Stimme, Ärger und Wut. Bei der Bearbeitung der Retroflektion empfiehlt er insbesondere die Arbeit mit dem Körper als sinnvolle Arbeitsweise, weil sie die fokussierte Aggression, deren Vermeidung das Kernstück der depressiven Dynamik bildet, mit hohem Gewahrsein verbindet. 


\subsubsection{Depression als Polaritätskonflikt, als eingeschränkte Ich-Funktion oder als der zurückgedrängte expressive Impuls}

Nach Perls Begriffen über unsere Persönlichkeit wird Depression als TopdogUnderdog-Konflikt, also „Konflikt von der Macht des Ohnmächtigen und der Ohnmacht des Mächtigen“ beschrieben. Dies kann als Polarität und Spaltung verstanden werden. Die Begriffe Topdog und Underdog könnten von Friedrich Nietzsches Gedankenfigur "Herr und Sklave“ abgeleitet sein. Der Topdog ist Kontrollierer, der autoritäre Rechtschaffende und Täter, seine Herkunftskonzeption ist Freuds Begriff „Über-Ich“. Er, als Manipulierter, sagt dem Underdog „Du sollst" oder „Du sollst nicht“. Der Underdog ist der Kontrollierte, Gemaäregelte und Opfer, aber er ist nicht gleichzusetzen mit Freuds Begriff „Es“. Er sagt als „ein Frustrator“: „Du hast Recht. aber...."(Blankertz \& Doubrawa 2005). Menschen versuchen in ihrem ganzen Leben diese Polarität zu balancieren und zu integrieren. Das ist unser Schicksal, da ein Kind erst durch „Erziehung“ eingeschränkt wird. Während des lebenslangen Dauerkonflikts verliert man die schöpferische Lebensenergie, insofern man in diesem Konflikt, ohne ihn zu integrieren, bleibt. Perls nannte diese Konstellation, das „neurotische Self-torture game“. Aber es gibt eine Lösung: „wenn Topdog und Underdog integriert sind, weiß man, was man will“ (Perls 1980).

Die beiden kämpfen, um die Kontrolle zu gewinnen, aber es gewinnt der Underdog immer durch Sabotage (Verrückt-Spielen, Zögern, Müdigkeit etc.), weil das Ziel vom Topdog perfekt, ideal und folglich unerreichbar ist, so dass der Underdog immer gegen den Topdog ärger und schlechtes Gewissen haben muss. Während der Topdog vom Underdog sein ideales Ziel verlangt, entwickelt der Underdog ein Selbstbild von Hilflosigkeit, Inkompetenz und Niedergeschlagenheit (Amendt-Lyon 1999), indem sich der Underdog schuldig fühlt und projiziert, wenn gegenläufige Impulse oder Aggression unterdrückt werden müssen. Solch ein Konflikt bleibt als unerledigte Gestalt und als eine Art Persönlichkeitsspaltung im Organismus, bis der Konflikt zwischen den Gegenpolen gelöst wird.

Wenn solch ein Konflikt nicht nur auf der intrapersonalen sondern auch auf der interpersonalen Ebene entsteht und sich ein entsprechendes Muster in der zwischenmenschlichen Beziehung entwickelt, spielt das eine Individuum die Rolle des Opfers und projiziert auf andere den Täterpart. Es bekämpft ihn entweder in sich selbst oder in seinem nach außen projizierten Gegenpol. Solch ein Konflikt auf interpersonaler Ebene findet man häufig in der Familie, wenn Eltern ihr Kind tyrannisch behandeln und weiterhin das Kind keine Gelegenheit hat, die 
Überforderung durch seine Eltern zu verdauen. Es muss stattdessen schlucken, ohne sich auszudrücken zu können. Dann muss das Kind seine Energie auf vielfache Weise ableiten, was die Möglichkeiten einer kontaktreichen Interaktion mit seiner Umwelt verringert. In diesem Fall findet Introjektion statt, bei der das Kind das Unverdaubare geschluckt hat. Das Kind betrachtet alles, was es nicht spontan erfährt, als schädlich. Entweder nimmt es die Nahrung in der Form an, in der sie ihm dargeboten wird, oder es spuckt sie wieder aus (Polster \& Polster 1975). Das Kind zieht sich die Elternkleidung an, versucht sich anzupassen und verfolgt die Norm „Sollen“, anstatt sowohl seine eigenen Bedürfnisse und seinen eigenen Wille als auch seine Energie und Kräfte zu spüren. Es hat ein Motto, „mir wird verweigert, wenn ich mich auf meine eigenen Bedürfnisse einlasse“. Es lebt ohne sich selbst zu spüren und ohne Wahlmöglichkeit, sondern lediglich mit der Anforderung des "Sollens". Bei Depressiven entstehen Introjektionen besonders in der Phase der „Mobilisierung der Energie".

Depression ist $\mathrm{zu}$ verstehen als eine eingeschränkte Ich-Funktion des Selbst (vgl. Mentzos 1984; Amendt-Lyon 1999), die später auf die Es-Funktion und die Persönlichkeit einschränkend einwirkt. Nach Mentzos geht es bei Depression um eine Ich-Hemmung, eine Ich-Einschränkung, ein Absinken der Aktivität und der Leistung und einen Rückzug von der Welt. Die Ich Funktion, „die sich als aktives Zugehen auf die Umwelt, als sich Identifizieren mit den eigenen Bedürfnissen und als SichBemühen, diese Interessen durchzusetzen, manifestiert" (Amendt-Lyon 1999, S. 152), ist beim Depressiven eingeschränkt. Sie ist verwandt mit der Ich-Funktion, die die Grenze des Ichs bildet.

Polster und Polster (1975) unterscheiden Grenzen in fünffacher Weise: Körpergrenze, Wertgrenze, Grenzen der Vertrautheit, Grenzen des Ausdrucks und Grenzen der Bloßstellung (vgl. S. 116-126). Die Körpergrenzen sind das Empfindungsvermögen oder die Funktion der Körpers, und bei Depression ist der Kontakt zu diesem wichtigen Teil des Selbst eingeschränkt wahrnehmbar. Als Handlungsmöglichkeit sind die Wertgrenzen bei Depression nicht vielfach, sondern unflexibel entwickelt, da depressiven Menschen mit ihrer Findigkeit und Initiative die Gegenwart des Systems nur eingeschränkt auszugleichen vermögen, sodass sie ihre Energie zur Gestaltung ihres Lebens nur gering mobilisieren können. Sie sind unfähig oder wenig mutig, ihre bekannte Welt zu verlassen, obwohl sich die Welt immer verändert und sie als Menschen imstande sind, sich zu verändern, so dass sie ihre Grenze der Vertrautheit oder der Veränderung nicht entfalten können. Bei Depressionen sind die Grenzen des Ausdrucks absolut gehemmt. 
Scharfetter (2002) nennt fünf Qualitäten beim basalen Aufbau des Ichs: Ich-Vitalität, Ich-Aktivität, Ich-Konsistenz und -Kohärenz, Ich-Demarkation und Ich-Identität. Dies sind basale Reifungsqualitäten und -stufen. Ihre ungestörte Abfolge und Integration bildet die Voraussetzung für die spätere „neurosefähigen Struktur“, die durch die ganzheitliche Qualität des Ich-Selbst-Erlebens gekennzeichnet ist. Auf ihrem Boden kommt es zur Differenzierung von Ich-, Es- und Persönlichkeitsfunktionen. Dieser frühe gestalttherapeutische Entwurf lehnt sich an das Freudsche Strukturmodell von 1923 an. Als physiologisch Erstes finden sich Es-Funktionen, dann Ich-Funktionen und als Folge der assimilierenden Erlebnisverarbeitung die Persönlichkeitsfunktionen. Die drei Funktionen bezeichnen, womit sich der leib-seelische Organismus im Kontaktprozess identifizieren muss, nämlich mit dem, was er braucht (Es-Funktionen), mit dem, was er tut bzw. nicht tut (Ich-Funktionen) und mit dem, was er ist (Persönlichkeitsfunktionen). Die Ich-Funktionen des Spürens, des BeachtungSchenkens, Pflegens und insgesamt des Sich-Auseinander-Setzens mit den existentiellen Gegebenheiten des Lebens halten also die Es- und Persönlichkeitsfunktionen aufrecht (vgl. Dreitzel 2004, S. 42-44).

Dreitzel unterteilt die Ich-Funktionen im Kontaktprozess in seinem Diagnostikmodell. In der 1. Phase (Vorkontakt) sind sie Brauchen, Wünschen und Wollen, in der 2.Phase (Orientierung und Umgestaltung) Wahrnehmen, Zugreifen, Zerlegen und Beseitigen, in der 3. Phase (Voller Kontakt) Sich-Hingeben, Genießen und Sättigung spüren, in der 4. Phase Einsinken lassen, Nachspüren und Bewerten und Sich Identifizieren.Die in dem Kontaktprozess spontan auftauchenden Gefühle und Affekte, die man in der Gestalttherapie „Kontaktgefühle“ nennt, sind die Funktionen des Selbst, wobei die Intensität der Kontaktgefühle von den Gegebenheiten des Organismus/Umwelt-Feldes abhängen (vgl. ebd. S.38-40). Dreitzel(2004) zeigt den Arbeitsweg mit dem Sprechkontakt, der Belebung der Motorik und der Sinne, und dem Beharren auf konkrete Aussagen.

In diesem Rahmen definiert er den depressiven Prozess, in dem die Grunderfahrung der Depression „Was immer ich erlebe, tue oder zum Ausdruck bringe; es macht keinen Unterschied“, da alles im depressiven Prozess als gleich-gültig erlebt wird wegen der Projektion. Darüber hinaus wird der biographische Hintergrund beschrieben: Die „Unmöglichkeit, Wut und Schmerz auszudrücken, sei es wegen starker Verbote und Strafen, sei es, weil niemand da war, der es hätte hören und aufnehmen können“. Dabei lassen sich Auswirkungen auf die Es-Funktionen bzw. Persönlichkeitsfunktionen wie folgt beschreiben (ebd. Kommentar zu Schaubild 10): 
(a) Auswirkungen auf die Es-Funktionen: Die Bedürfnisse werden gespürt, aber die Entscheidungsfähigkeit ist durch Sinnlosigkeitsintrojektion stark beeinträchtigt, so dass es oft bei Tagträumen bleibt.

(b) Auswirkungen auf die Persönlichkeitsfunktionen:

- Es fehlt an Lebensmut. Der Körper wird überwiegend als beschwerlich erlebt. Der Tod wir als Ausweg gesehen, wobei es aber meist an Energie für einen Suizid fehlt. Gesellschaftliche Anpassung, wobei die Verantwortung gern projiziert wird.

- Starke Sinnlosigkeitsgefühle.

Die folgenden Autoren haben zwar kein gestalttherapeutisches Selbstverständnis, sind aber mit ihrem Modell ergänzend und diskussionswürdig.

Mentzos (vgl. 1984, S. 182-186) stellt die drei Unterschiede zwischen Trauerreaktion und pathologisch depressiven Prozessen dar. Hier referiere ich über die Unterschiede. A)Bei der Depression ist das Ausma§ der eingeschränkten Ich-Funktion des Selbst, was in der Psychoanalyse Ich-Hemmung genannt wird, noch extremer als bei der Trauerreaktion. B) Bei der Depression spielt Aggression, und insbesondere Autoaggression, die als Retroflexion in der Gestalttherapie verstanden wird, eine große Rolle. Die Aggression hängt mit Besonderheiten des Grundkonfliktes und strukturellen Besonderheiten des Depressiven zusammen. Das heißt, dass die massiven Aggressionen nicht in die Tat umgesetzt, sondern autoaggressiv, also retroflektiert abgewehrt werden: Selbstvorwürfe, Anschuldigungen, Nahrungsverweigerung, Selbstverstümmelung, Suizidalität, schließlich Selbstmord. In der normalen Trauerreaktion ist Aggression enthalten, aber nicht als Autoaggression. C) Der Objektverlust gibt den Anlass zur Entwicklung einer Depression. Bei der Trauerreaktion entsteht eine massive Introjektion des verlustig gegangenen Objekts als Kompensation des Verlustes und als Erinnerung, damit der Verlust irgendwie rückgängig gemacht wird. Im Unterschied dazu ist aber der Vorgang der Introjektion innerhalb des depressiven Symptoms primitiv und undifferenziert, sodass er nicht zur einer Erleichterung und Lösung, sondern im Gegenteil zu größeren Schwierigkeiten führt, weil der Organismus seine Aggression gegenüber dem introjizierten Gegenstand nicht auszudrücken vermag.

Petzold (1995) stellt ein Repressionsmodell der Pathogenese in der Integrativen Therapie dar. Er meint, dass Depression entsteht, wenn der expressive Impuls durch externale Repression (negative Sanktionierung, Bedrohung, Kritik) zurückgedrängt wird. Nach dem Modell werden zwei kommunikative Zyklen begonnen: Wachstums- 


\section{Gestattherapie und Depression}

und Progressionszylus und Depressions- und Regressionszyklus. Wachstums- und Progressionsprozess beginnt bei: positiver Impression $\rightarrow$ Erregung $\rightarrow$ Innenresonanzen $\rightarrow$ Expression $\rightarrow$ Außenresonanz und dann wiederum Impression usw. Beim Regressions- und Depressionsprozess kann die Emotion durch negative Impression, externale Repression und negative Außenresonanz nicht zum Ausdruck gelangen. Der verdrängte expressive Impuls führt wiederum zur Selbstrepression als Retroflexion. Es kommt dann als Folge dieser Dynamik häufig zur Depression und/oder zu psychosomatischen Reaktionen. Da Emotionen als Resonanz auf Einflüsse der aktualen Umwelt ausgedrückt werden dürfen, ermöglicht offenes Ausdrücken emotionaler Impulse die Reduzierung emotionaler Spannung (vgl. S. 254-257).

\subsubsection{Depression als dysfunktionale sekundäre Emotion aus der Perspektive} „Emotion-focused Therapy $(E F T)^{7}$ “ nach Greenberg und seinen Mitarbeitern

\subsubsection{Der Ansatz EFT und emotionale Schemata}

EFT ist eine neohumanistische Behandlungsform, in der eine kreative Verbindung oder eine Integration zwischen einer klientzentrierten Beziehung und einem prozessdirektiven Stil der Gestalttherapie hergestellt wird, die wissenschaftlich fundiert ist(im Sinne von Grawe 1997) und von der Forschung bestätigt wurde. Die theoretischen Grundlagen der EFT sind die Emotionstheorie, der dialektische Konstruktivismus und die Prozessorientierung sowie die Grundprinzipien des Behandlungsmodells. Die wesentlichen humanistischen Werte (vgl. Tageson 1982, zit. nach Elliot 1999), die in EFT integriert sind, sind empathische Einstimmung, authentische zwischenmenschliche Beziehung, kontinuierliches psychisches Wachstum während der gesamten Lebensspanne, sowie Selbstbestimmung und Selbstgerichtetheit (vgl. Elliot 1999, S. 203).

Die Forscher gehen davon aus, dass „Emotionen grundsätzlich adaptiv sind und dem Organismus dazu dienen, komplexe, situative Informationen schnell und automatisch $\mathrm{zu}$ verarbeiten mit dem Ziel, Handlungen $\mathrm{zu}$ vollziehen, die die wichtigsten BedŸrfnisse des Organismus erfüllen“ (Elliot 1999, S. 204). Der Ausdruck von Emotionen wirkt somit grundsätzlich kommunikativ und reguliert die soziale Interaktion. Am besten lassen sich Emotionen als komplexe Synthesen von Elementen verstehen, zu denen der primäre Affekt (in Form von Empfindung und Physiologie), die Motivation (in Form von Bedürfnissen und Anliegen) und die Kognition (in Form

\footnotetext{
${ }^{7}$ In Deutsch: auch prozess-erlebnisorientierte Therapie (PFT)
} 
von Bewertungen) zählen. Das heißt, dass bewusst erlebte Gefühle eine komplexe Synthese zahlreicher Arten von Information aus vielen verschiedenen Quellen sind. Sie umfassen mindestens drei messbare Systeme nach Lang (1984) und Leventhal (1984): ein physiologisch basiertes expressiv-motorisches System, ein semantisch basiertes emotionales Gedächtnissystem und ein verbal basiertes konzeptuelles System. Durch die komplexe Integration dieser drei Verarbeitungsebenen entsteht emotionale Erfahrung. Die emotionalen Zustände werden überwiegend durch Aktivierung komplexer emotionaler Schemata erzeugt. Diese Zustände- oder Selbstorganisationen dienen dazu, auf das Handeln vorzubereiten und die kognitive Verarbeitung $\mathrm{zu}$ beeinflussen. In der EFT gelten Affekte als zentaler Bestandteil menschlichen Seins, der die Verbindung zwischen Selbst und Umgebung herstellt und das Selbsterleben organisiert, und der das wichtigste Mittel der Selbstregulation und Kommunikation für Kinder ist. Gefühle sind in der EFT der Punkt, an dem Geist, Körper, Umgebung, Kultur und Verhalten zusammentreffen. Kognition wird eindeutig und unmittelbar von der Emotion beeinflusst und ist nur dann wichtig, wenn sie mit Gefühlen verbunden sind (Greenberg, Rice \& Elliot 1993, 2003, S. 91-96).

In diesem Ansatz hat deswegen die Arbeit mit Gefühlen, genauer gesagt: emotionalen Schemata, einen zentralen Stellenwert. Ein Schema definiert Bartlett (1932, S. 201, zit. nach Greenberg, Rice \& Elliot 1993, 2003. S110) „als eine aktive Organisation früherer Reaktionen oder früherer Erfahrungen, die bei jeder gut angepassten organismischen Reaktion wirksam sind“ und ein emotionales Schema ist "eine komplexe, nicht bewusstseinsfähige Struktur, die gesundes Funktionieren von Erfahrungen und Verhalten lenken oder stören kann“ (Strümpfel 2004, S. 363). Für das psychische Wohlbefinden von Menschen entscheidend sind jene Schemata, die das emotionale Erleben des Selbst in der Welt repräsentieren, so dass sie für eine Therapie insbesondere für EFT von zentraler Bedeutung sind. Nach Greenberg, Rice und Elliot (1993) ist ein wichtiger Aspekt von emotionalen Schemata,

„dass sie die Kognition zwar einbeziehen, über die rein repräsentationale Kognition jedoch hinausgehen, indem sie Emotion, Motivation und Beziehungshandeln einschließen. Sie sind nicht einfach nur konzeptuell und klassfikatorisch, sondern verkšrpern die Organisation von Sets von Antizipationen und Reaktionen. Das emotionale Schema der Unzulänglichkeit wird entweder außerhalb des Gewahrseins aktiviert, oder es ist besonders gut zugänglich und steuert das Verhalten antizipatorisch. Deshalb geht es in einer Therapie nicht darum, rein kognitive Schemata durch Geltendmachen von 


\section{Gestattherapie und Depression}

Venunftgründen oder mit Hilfe von Beweisen zu verändern (Beck 1976; Ellis 1962), sondern um die Veränderung der komplexen, kognitiven, affektiven, motivationalen und relationalen Komponenten des Handelns, die mit bestimmten emotionalen Schemata verbunden sind" (S. 111-112).

Diese emotionalen Schemata funktionieren je nachdem adaptiv, also anpassungsfähig oder maladaptiv also dysfunktional. Der Therapieprozess nach diesem Ansatz zielt darauf ab, den Zugang zu emotionalen Schemata unter therapeutischen Bedingungen zu finden, die entweder einen vollständigeren Ausdruck der Emotionen ermöglichen oder diese restrukturieren (vgl. Elliot 1999, S.205), nicht aber darauf, „unbewusste und verdrängte oder abgewehrte und geleugnete Emotionen $\mathrm{zu}$ erschließen und $\mathrm{zu}$ interpretieren. Denn Emotionen werden nicht gespeichert, sondern rekonstruiert, wobei die Rekonstruktion davon abhängt, wie die Information augenblicklich beurteilt wird und organisiert ist und ob die betreffenden emotionalen Schemata augenblicklich motiviert sind oder nicht" (Greenberg, Rice \& Elliot 1993, 2003, S. 115).

Die emotionalen Schemata unterscheiden sich nach vier Arten emotionaler Prozesssequenzen (vgl. Elliot 1999; Greenberg \& Safran 1989; Greenberg \& Paivio 1997): primär adaptive, emotionale Prozesse, - maladaptive, emotionale Prozesse, sekundär emotionale Prozesse - und instrumentelle, emotionale Prozesse. Primär adaptive emotionale Prozesse sind ungelernt und direkte Reaktionen auf die Situation. Wenn diese Prozesse auf die auslšsende Situation, die von früheren unerledigten Erfahrungen abhängig sind, abgebrochen werden, entstehen maladaptive emotionale Reaktionen. Sekundär emotionale Prozesse sind Reaktionen auf die primär adaptive Emotion, was zur Folge hat, dass diese durch sekundäre Emotionen ergänzt wird. Adaptive Emotion wird durch eine selbst- bzw. auf andere fokussierte Reaktion auf die primäre Reaktion verschleiert. Instrumentelle emotionale Prozess sind ein simulierter Ausdruck emotionaler Zustände, um andere zu beeinflussen oder zu kontrollieren. In der Behandlung müssen diese vier Prozesse differenziert und muss mit ihnen unterschiedlich umgegangen werden (vgl. Greenberg \& Paivio 1997).

Nach Elliot (1999, S. 206) müssen primär adaptive Emotionen erschlossen und zugelassen werden, maladaptive Emotionen werden mit der Strategie, nach der der Zugang $\mathrm{zu}$ diesen gefunden und zugrunde liegende emotionalen Schemata zur Neustrukturierung exploriert werden, untersucht. Sekundäre Emotionen erfordern mit empathischer Exploration die Entdeckung der darunter liegenden primären Emotionen. Für die Untersuchung der instrumentellen Emotionen sind ihre interpersonalen Bedeutungen und ihre motivationalen Grundlagen zur Entlastung 
wichtig.

\subsubsection{Depression als sekundäre emotionale Prozesse}

Depressive Affekte werden einschließlich Gefühlen der Hoffnungslosigkeit und Motivationslosigkeit als sekundäre emotionale Reaktionen auf Schwierigkeiten in darunterliegenden emotionalen Prozessstrukturen verstanden (vgl. Greenberg \& Safran 1987; Greenberg \& Paivio 1997; Greenberg \& Watson 1999). Die depressiven Klienten werden am Anfang der Behandlung oft in hohem Grade beunruhigt und äußern sich leicht und ausgiebig über die Sachen, die sie stören. Jedoch drücken sie normalerweise nicht die primäre Traurigkeit über einen Verlust aus, sondern können sich verärgert und hilflos fühlen und versuchen, Unterstützung oder eine Kombination von diesen Gefühlen zu erwähnen. In diesen Situationen sind Interventionen, die darauf abzielen, einfach die Traurigkeit zuzulassen und anzunehmen, nicht nützlich, da die Traurigkeit komplexe Inhalte und dynamische Merkmale hat.

Greenberg und Paivio (1997) unterscheiden „primär adaptive Traurigkeit", „primär maladaptive Traurigkeit", "Sekundär-Traurigkeit und Depression" und „Instrumentelle Traurigkeit". Für die passende Intervention muss der Therapeut zwischen Traurigkeit und anderen emotionalen Erfahrungen und zwischen den Primär-, Sekundär- und instrumentalen Expressionen der Traurigkeit unterscheiden (S. 164-193). Im Folgenden referiere ich die Merkmale und die Funktionen verschiedener Traurigkeiten und Depression von Greenberg und Paivio (1997) und Greenberg, Rice \& Elliot (1993).

(a) Primäre adaptive Traurigkeit: Primäre adaptive Traurigkeit ist ein Zustand, der häufig in der Therapie als kurzer Moment erscheint, der in einem komplizierten psychologischen Prozess eingebettet ist. Sie wird durch eine Art momentaner Auslieferung gekennzeichnet und ist von Schuldgefühlen frei. In den frühen Stadien der Therapie ist primäre adaptive Traurigkeit wie Zorn, häufig entweder undifferenziert oder unterdrückt. Die offensichtlichen Markierungen von unterdrückter Traurigkeit und emotionalen Schmerzen schließen Intellektualisierung oder Beschädigungen und verspannte Muskeln ein. Dazu gibt es eine Abneigung, ausdrücklich über Schmerzen zu weinen oder Schmerz zuzulassen, aus Furcht oder Sorge darüber, durch die Traurigkeit überwältigt zu werden. Der Unterschied zwischen der primären Traurigkeit und der sekundären Depression, Traurigkeit oder Hilflosigkeit basiert auf der Kenntnis der Situation und der Person, sowie den verbalen 


\section{Gestattherapie und Depression}

und nonverbalen Spuren, einschlie§lich der Qualität der Stimme, des Gesichtsausdrucks und der Verhaltensweise. Vor allem ist die primäre Traurigkeit - anders als die Hilflosigkeit und Depression - ein lebhafter Zustand, der zur Veränderung führt. Die undifferenzierte Traurigkeit wird teilweise durch die auslösende Situation erkannt. Z.B. in der Situation des Verrats, wird Traurigkeit mit Zorn gemischt. Beim Trauma ist sie mit Angst, bei Misshandlung ist sie mit Ärger, Angst und Scham gemischt. Alle diese Gefühle können primär sein, und jedes sollte vollständig erfahren und ausgedrückt werden. Interventionen, die die Traurigkeit von diesen anderen Gefühlen unterscheiden, konzentrieren sich darauf, welche Situation sie auslösen oder ausgelöst haben.

(b) Primär maladaptive Traurigkeit: Die Einschätzung davon, ob primäre Traurigkeit adaptiv (anpassungsfähig) ist oder maladaptiv (dysfunktional) ist, ergibt sich aus dem Kontext und dem Inhalt der Traurigkeit und zeigt sich mit der Zeit. Wenn die Situation den Verlust und die Verletzung des Selbst einbezieht, ist der erste Schritt, die Erfahrung der Traurigkeit und ihren Ausdruck, der zu einer Auflösung des Gefühls führt, zu erleichtern. In bestimmten Fällen scheint sich jedoch das Gefühl nicht aufzulösen, und die Person wiederholt das gleiche Gefühl immer wieder ohne irgendeine wahrnehmbare €nderung, entweder bezüglich der Qualität oder in der Intensität. Andernfalls können Traurigkeit und die Tränen eine dysfunktionale Qualität von Auflösung (Fragmentation) bekommen, sowie von Furcht oder hilfloser Abhängigkeit mit einer Unfähigkeit, eine innerliche Kohärenz oder lösungsorientierte Handlungskompetenz wieder $\mathrm{zu}$ finden. Dies legt Probleme über mangelnde Bewältigung von Leid und Schwäche nahe. In diesen Fällen fangen der Therapeut und der Klient an, zu verstehen, dass diese Traurigkeit dysfunktional ist. Ihre Arbeit führt zu einer Umstrukturierung dieser Erfahrung.

Pathologische oder schwierige Reaktionen gegen das Leid sind Beispiele der dysfunktionalen primären Traurigkeit. Die Person ist nicht imstande, mit dem Verlust fertig zu werden. Häufig werden in dieser Situationen der Ausdruck des ungelösten Zornes und starke Schuldgefühle empfunden. Einige Leute fühlen sich bei dem Verlust unregelmäßig traurig und vermeiden dennoch, sich konkret auszudrücken. Insoweit ungelöste Gefühle nicht verarbeitet werden, können sie an dem nicht nur emotionalen sondern auch psychosomatischen Prozess immer wieder beteiligt werden.

Eine Intervention kann zur Folge haben, dass das Schema der Gefühle zugänglich wird und die Schwierigkeiten der Person mit dem Annehmen des Verlustes behoben werden. Schließlich ist paradoxe Traurigkeit eine Art der dysfunktional primären Traurigkeit. 
Dabei sind Gesten von Freundlichkeit und Weichheit typisch, Gesten von anderen, einschließlich des Therapeuten. Es ist, als ob die Freundlichkeit eine tiefe Sehnsucht, Deprivation und Abhängigkeit anzeigt. EFT beinhaltet, das Schema der Kerngefühle oder das Gefühl zum Selbst alleine zugänglich zu machen, die Schmerzen und die Bedürfnisse zu bestätigen, und dann ein Schema, das sich auf die Gefühle bezieht, zu ändern. Das wird teilweise durch eine korrigierende emotionale Erfahrung mit dem Therapeuten und teilweise auch durch die als Alternative verfügbaren Ressourcen vollendet.

(c) Sekundäre Traurigkeit und Depression: Es ist wichtig zu unterscheiden: zwischen Tränen der primären Traurigkeit und Schmerzen und Tränen, die die Reaktionen auf andere emotionalen Erfahrungen sind, wie Frustration, Hoffnungslosigkeit oder Zorn. Letzteres tritt auf, wenn sich der Klient chronisch in die Verletzung und die Traurigkeit stürzt, weil er sich immer verärgert fühlt. Sekundäre Reaktionen sind erkennbar durch verbale Spuren und ihre zeitlichen Reihenfolge. Beispielweise, wenn Zorn erst ausgedrückt wird, wird er von Tränen gefolgt. Exploration auf diese Erfahrungen in der Therapie deckt auf, dass sich die Tränen durch kognitiv-affektive sekundäre Prozesse ergeben, wie ein Vorgefühl des Verlustes oder der Ablehnung, die aus der Furcht, dem Leiden und den traurigen Gefühlen resultieren.

Die meisten normalen SekundŠrreaktionen, die mit Traurigkeit verbunden sind, sind Depressionen, mit einer Art generalisierter Hoffnungslosigkeit anstatt echter Annahme des Verlustes. So drücken Depressive die Resignation über den Verlust oder die Verletzung aus, wie etwa durch Selbstkritik und Aussagen mit „Sollte“. In diesem Fall ist Traurigkeit sekundär zu einer komplex kognitiväaffektiven Sequenz geworden. Wenn die Depression aus einer heftigen, primären Traurigkeit aus einem Verlust oder aus einer anderen primären Erfahrung resultiert, dann zielt die Behandlung darauf ab, dass die deprimierenden, sekundären, depressiven Reaktionen vor allem im zugrundeliegenden kognitiv-affektiven Prozess erkannt werden, und dass das schmerzliche primäre Gefühl bestätigt und erfahren wird. Diese Interventionen helfen dem Klienten, in die hoffnungslosen, schlechten Gefühle hineinzugehen, um unerledigte Geschäfte abzuschließen und um innere Ressourcen zu alternativen Handlungen zugänglich zu machen, indem ein spezifischer Verlust oder eine Verletzung bestätigt und angenommen wird. Der Klient ist dann in der Lage, sich auf neue Interessen zuzubewegen. 


\section{Gestattherapie und Depression}

(d) Instrumentelle Traurigkeit: Instrumentelle Expression der Traurigkeit wird beobachtet, wenn Leute, die sich hilflos oder abhängig fühlen, weinen. Sie werden abwertend gekennzeichnet als "Jammerer“. Wenn Tränen eine Form des Widerspruchs sind, wenn man sich schlecht fühlt und schlecht behandelt wurde, dann ist es ein Ausdruck dafür, dass man zeigt, dass man Sympathie, Unterstützung oder das Verstehen von anderen braucht. Die Betroffenen können - oder können möglicherweise nicht - die instrumentale Funktion ihrer Tränen, die echte Bedürftigkeit ausdrücken können, zuordnen. Meist resultiert diese Traurigkeit nicht aus der Erfahrung eines Verlustes. Die Funktion der instrumentalen Tränen ist, die Aufmerksamkeit von anderen zu erhalten, oder wie eine Ausrede dafür, um nicht zu ändern, was gerade passiert ist. Aber man bekommt nicht immer die gewünschte Unterstützung von anderen. Es ist angebracht, die Funktion der instrumentellen Tränen empathisch zu erleben und zu deuten, um die zugrunde liegenden Motivationen und die Bedürfnisse zugänglich zu machen. Es gibt dem Klienten eine Chance, seine Bedürfnisse zu erkennen.

\subsection{Die gestalttherapeutische Behandlung von Depressionen}

\subsubsection{Merkmale der Behandlung von Depressionen}

\subsubsection{Erfahrungsorientierte und emotionsfokussierte Behandlung „Hier und Jetzt}

In der klassischen Gestalttherapie wurde die Behandlung von Depression nicht in einer spezifischen Weise entwickelt. Im Rahmen der gestalttherapeutischen Behandlung findet die Veränderung beim Klienten dahingehend statt, dass Emotionen mit Hilfe eines verschärften Gewahrseins im Hier und Jetzt erlebbar werden, anstatt sie zu vermeiden. Gestalttherapeuten gehen davon aus, dass Veränderung stattfindet, wenn man so Imstande des „Selbst-zu-Sein“ ist, anstatt sich zu bemühen, jemand anderer sei zu Wollen. Ein akzeptierender, gesunder und positiver Kontakt zum Selbst gilt als zentrales Anliegen. Wenn man sich damit identifiziert, wer man tatsächlich ist, einschließlich der innerlichen Konflikte und des dysfunktionalen Verhaltens in einer Haltung des Selbstrespekts, findet eine fundamentale Veränderung statt. „Sehen, was ist, verändert“. Das ist die Theorie des Paradoxes der Veränderung (Beisser 1970). Solch eine Haltung gilt auch für den Therapeuten, besonders in der therapeutischen Beziehung, wobei die Haltung der "Akzeptanz" erfahrbar sein soll, vom Therapeuten zu sich selbst und zum Klienten, und zwar so, wie er gerade ist. Es soll eine basale 


\section{Gestattherapie und Depression}

Unterstützung für den Klienten sein, sodass der Klient aus der Sicht und der Haltung des Therapeuten die Haltung "Selbstakzeptanz" lernen und sie weiter in seinem Leben anwenden kann.

Die Methode der Gestaltarbeit schließt 1) phänomenologische Fokussierung und das Experiment zur Erhöhung des Gewahrseins ein und 2) die Beziehung, die auf dialogischem Kontakt basiert (Yontef 2007, S.18), damit ist auch eine Selbst -Rekognitionsstrategie gegeben, Selbstrespekt und die Wertschätzung der Erfahrung des Klienten, wofür aktives Experimentieren gefördert wird. Besonders kann die

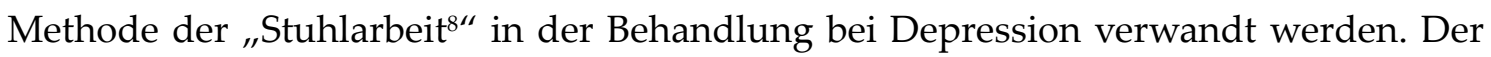
Stuhl dient lediglich als Projektionsfläche innerer Bilder. Fritz Perls sah die Depression als innerliches Konfliktgeschehen an, wie es oben beschrieben wurde. Die Arbeit mit dem leeren Stuhl bzw. mit zwei Stühlen wird von Greenberg und seinen Mitarbeitern unterschiedlich, je nach Fall, in der Behandlung von Depression verwandt. HartmannKottek (2004) schlägt die Arbeit mit mehren Stühlen vor, sofern eine Differenzierung in Vorder- und Hintergrund oder von ambivalenten Selbst- oder Fremdbildern sinnvoll erscheint.

Der Charakter der gestalttherapeutischen Behandlung ist existentiell, experientiell und experimentell, wie Laura Perls erwähnt (vgl. Rosenfeld, 1978). Diese Elemente sind miteinander verbunden in dem Kontext (Hier) und dem Kontinuum (Jetzt) mit den phänomenologischen Methoden. Tiefes Gewahrsein ${ }^{9}$ statt bloßer Gedanken, ausreichende Experience statt Erklärung und spontanes Experiment statt Interpretation haben einen hohen Stellenwert in der gestalttherapeutischen Arbeit. Zwei Hauptziele in der Gestaltarbeit sind Gewahrsein und Kontakt (Harmann 1990). Die beiden sind in der Gestalttherapie sowohl terminus a quo als auch terminus ad quem. Durch Gewahrsein wird ein spezifisches oder unerledigten Bedürfnis in dem Organismus verdeutlicht, sodass Form und Klarheit dessen, was von früherer Erfahrungen unerfüllt verdrängt geblieben ist, angenommen und Gefühle geweckt werden und auf vollen Ausdruck und volle Erfüllung drängen. Gefühle sind in der Gestalttherapie eigentlich „Kontaktgefühle, Mittel des Erkennens und Träger von Informationen über den Zustand des Organismus/Umwelt-Feldes“ (Dreitzel 1995, S. 493), da Gefühle ein kognitives Moment, eine Einschätzung der Situation, die zugleich informiert und motiviert, enthalten und durch Information zum Handeln motivieren.

\footnotetext{
${ }^{8}$ Die Methode „Stuhlarbeit“ und ihre Funktion in der Behandlung bei Depression werden im nächsten Untertitel erklärt.

9 Yontef (1984) betont, dass das Ziel der Gestalttherapie immer Gewahrsein und nur Gewahrsein ist (S. 294).
} 
Deshalb sind die Qualität des Kontakts (oder der Kontaktgefühle) von Klienten immer wichtige Hinweise für Gestalttherapeuten, wie, wo und in welcher anderen Form der Kontakt im Prozess entsteht, entwickelt und blockiert wird. „Ein zentrales Ziel der Gestalttherapie ist die Wiederbelebung und Verfeinerung des emotionalen Sensorismus durch aufmerksameres Gewahrsein“ (Dreitzel 1995, S. 501), da Emotion eine entscheidende Spur zum Kontakt und ein endgültiger Hinweis für Therpeuten zur Intervention ist. Emotion ist nicht nur seelisch sondern auch körperlich mit dem Zustand eines Menschen verbunden, so dass bei der Wiederbelebung und Verfeinerung des emotionalen Sensorismus in der Gestalttherapie Emotionen auf sensormotorischer Ebene aufmerksamer wahrgenommen und ausgedrückt werden. Dafür beinhaltet Gestalttherapie die phänomenologische Haltung und Methode hinsichtlich der „Körperprozesse“ (Kepner 1987) oder die „Wahrnehmungsübung“ (Stevens 1971) in dem ganzheitstheoretischen Hintergrund, wobei der Körperprozess nicht vernachlässigt sondern als bevorzugtes Phänomen respektiert wird.

Solche Behandlungsweisen und Methoden werden in der EFT bei Depression mehr verfeinert. Die Interventionen der EFTs bei Depression, die sich sowohl aus der klientenzentrierten Therapie als auch aus der Gestalttherapie herleiten, konzentrierten sich auf spezifische, kognitiv-affektive Determinanten emotionaler Probleme, um Zugang zu den darunter liegenden emotional bedingten Schemata zu finden, die zu der Störung beitragen und um diese neu zu strukturieren (Greenberg \& Watson 1999, S. 1122). Neben der Verwendung des „empatischen Spiegelns“, was von der klientenzentrierten Therapie für die Intervention PETs adoptiert wurde, eine unnötige Adoption, wenn man sich als Gestalttherapeut im „Ich-Du-Verständnis“ Martin Bubers sicher fühlt, was Leslie Greenberg, nach seiner eingenen Angaben in seiner Gestaltausbildung nicht vermittelt worden war (persönliches Gespräch/ IGW Kongress/2004), wurden der Dialog zwischen zwei Stühlen, um Selbstkritik oder Spaltung aufzulösen, und der Dialog mit dem leeren Stuhl, um mit unerledigten Geschäften und Abhängigkeitsproblemen umzugehen, genutzt. Außerdem wurde gezielte Ausdrucksunterstützung verwandt, um dem Klienten zu helfen, sein Abdriften in die Depression und andere verwirrende Reaktionsweisen zu identifizieren und besser zu verstehen (vgl. Greenberg \& Watson 1999).

Der Ansatz EFTs schließßt die Veränderung der dysfunktionalen Kognition in der Behandlung für Depression ein. Jedoch werden Kognitionen zur Veränderung nicht direkt herausgefordert, weil diese als "Nebenprodukte" von den Schemata der komplizierten Gefühlen und der Kernerfahrungen angesehen werden. Eher wird 
Veränderung vollendet, indem man Gefühle der Hoffnungslosigkeit zugänglich macht und völlig erfährt, und dann werden die kognitiv-affektiven Bestandteile der Depression verändert, während die zugrundeliegende anpassungsfähige primäre Erfahrung zugänglich gemacht und völlig wieder erlebt wird und dabei die Ressourcen entdeckt und verfügbar gemacht werden. Diese fungieren als Umstrukturierung des Schemas des Kerngefühls, das sich dysfunktional auf das Selbst bezieht, und sollen zur Veränderung dysfunktionaler Kognition führen (vgl. Greenberg \& Paivio 1997, S. 167-168).

Die Behandlungsprinzipien der EFTs (Greenberg, Rice \& Elliot 1993; 2003) sind unter den zwei globalen Prinzipien, nämlich Beziehungprinzipien und Aufgaben betreffende Prinzipien, sortiert. Die Beziehungsprinzipien fördern eine therapeutische Beziehung, indem (a) empathische Einstimmung, (b) therapeutische Bindung und (c) gemeinsame Arbeit an Aufgaben zutage tritt. Die Aufgaben betreffenden Prinzipien fördert an bestimmten Therapieaufgaben, während (d) erlebnisorientierte Verarbeitung, (e) Wachstum und Entscheidungsfähigkeit und (f) Lösen von Aufgaben unterstützt werden. Dabei schlagen Greenberg und Paivio (1997) die drei Prinzipien der Behandlung vor, die insbesondere für die Arbeit mit Traurigkeit einschließlich Depression sind (S. 168ff.). Die Prinzipien sind erstens die unmittelbare Aufmerksamkeit für das Erlebnis, das zur kšrperlichen Wahrnehmung führt, zweitens „Präsenz" und drittens Intensivierung des Erlebnisses. Rice und Greenberg (1990) unterscheiden drei Basistypen von Klientenaktivitäten: Experiential Exploration bei Wiedererleben der unerledigten Geschäfte im Hier und Jetzt, aktiver Ausdruck (eventuell mit der Stuhlmethode) der emotionalen Prozesse und interpersonales Lernen in therapeutischer Beziehung.

Da Depression ein innerlich bewegendes Erlebnis ist, das durch Passivität oder Untätigkeit gekennzeichnet wird, müssen die aktiven Interventionen mit empathischen Inhalten, die diesen ruhigen Zustand hervorheben können, gestaltet werden. Diese Interventionen bestehen aus: der Aufmerksamkeit auf körperliche Wahrnehmungen, Imagination und Symbolisierung der Bedeutung des Leidens, etc. So werden die körperlichen Spuren genutzt. Durch diese Interventionen wird die Experience des Klienten vertieft. Die Interventionen zur Intensivierung der Experience zielt auf die Bewältigung des Vermeidens der Gefühle und auf tiefes Gewahrsein dessen, was schwierig abzulehnen ist, ab. Traurigkeit oder Depression kann mit dem Nutzen von Metapher, evokativer Reflexion, körperlicher Erfahrung vertieft werden.

Da der emotionale Prozess beim Klienten mit Depressionen während Arbeit sehr schnell verläuft, z.B. von Traurigkeit zu Zorn, zu Angst und zu Ablehnung (und dann 
zurück zu Traurigkeit) darf der Therapeut weder voreilig zu einem Schluss noch zu einer raschen Annahme des Leidens sowie zur Entlastung des Leidens zwingen. Stattdessen muss er eine Haltung zur „Präsenz“ als Stütze schaffen, damit der Klient mit der Stütze freiwillig in die mehrschichtigen Gefühle tief hineingeht oder zurück zum Hier und Jetzt. Solche Haltung sichert für den Klienten einen sicheren Raum, wo er sich im seinem eigenen Tempo erforschen kann. Diese Haltung ist eine Richtschnur für den Klienten, die als Begleitung und als Rettung funktionieren kann, indem er mit ihr als Begleitung in unklare Tiefen springen und mit ihr als Verbindung aus der Tiefe zurückkommen kann, in der man sich gerade erforscht hat, ohne den Rückweg zu verlieren und sicher.

Die Behandlungen der Gestalttherapie und der EFTs haben beide ihren Schwerpunkt auf der Arbeit mit Emotion im Hier und Jetzt. Depression kann als unkontrollierbarer Ausdruck der Traurigkeit und als eine Reaktion gegen Auslöser verstanden werden. Die depressive Person reagiert nicht adäquat, verhindert wiederum ihre Fähigkeit zur kreativen Anpassung. Das Gefühl „Hoffnungslosigkeit“ ist eines der überwiegend schlechten Gefühle, die mit Traurigkeit und Leiden verbunden sind, und die generalisierte Hoffnungslosigkeit ist bei der Erfahrung der Depression zentral. Die Arbeit mit dem Gefühl „Hoffnungslosigkeit“ ist damit verbunden, Entmutigung zu überwinden, indem eine Ressource, die den Wunsch und den Mut weiter zu liefern vermag, zugänglich gemacht wird. Die Veränderung tritt dann auf, wenn Hoffnungslosigkeit völlig erfahrbar gemacht wird, anstatt sie zu vermeiden. Eine solche Haltung bezieht sich auf "the paradoxical theory of change“ von Arnold Beisser (1970), was zur Behandlung in der Gestalttherapie enorm beiträgt.

Häufig ist es schwierig für Klienten, sich dies antun zu lassen, weil sie soviel die Bemühungen aufgewandt haben, sich vom depressiven und hoffnungslosen Gefühl zu stoppen. Insofern das Gefühl „Hoffnungslosigkeit“ in der sicheren Atmosphäre tief erfahren und erforscht wird, anstatt vermieden zu werden, wird es sich in die primäre emotionale Erfahrung verlagern, um die spezifische Erfahrung, die die Hoffnungslosigkeit erzeugt hat, zu akzeptieren und die Ressource adäquat zu mobilisieren (Greenberg \& Paivio 1997, S. 190). Gestalttherapeuten sowie Therapeuten EFT vertrauen auf die Ressourcen des Klienten. Sie werden aktiviert, wenn der Klient ausreichend mit dem Moment des Hier und Jetzt in Kontakt kommt und akzeptiert, was gerade passiert. Solche Ressourcen werden spontan aktiviert und tragen aktiv zur Veränderung der dysfunktionalen Kognition und der Gefühle bei. Dabei gibt es Kontraindikation: Wenn der Klient das Gefühl der Suizidalität und das Risiko der Selbstverletzung oder des Selbsthasses hat, wird die Arbeit mit dem Gefühl 


\section{Gestattherapie und Depression}

Hoffnungslosigkeit für den Klienten als zu gefährlich erlebt, sodass die intensiven Gefühle direkt auf sich selbst gesteuert werden (vgl. Greenberg \& Paivio 1997, S. 191).

\subsubsection{Die Verwendung der "Stuhltechnik“}

Die „Stuhltechnik“ hat Perls von Monodramatechnik, die er von Moreno (1948) gelernt hat, kreativ in der Gestalttherapie in verschiedenen Variationen, z.B. der „Hot -Seat“ Technik, der „Technik mit leerem Stuhl“ und der „Technik mit zwei oder mehreren Stühlen", mit dem Ziel ggf. zur Basis eines Lernprozesses von Selbstwahrnehmung oder zur Auflösung eines Konflikts verwandt, damit der Klient die isolierten Anteile von sich neu integrieren kann. „Die Arbeitstechnik mit Stuhl“ ist nicht nur die berühmteste für die Gestalttherapie, sondern erwies sich auch als effektiv bei empirischen Forschungen in verschiedener klinischen Behandlung, besonders von Leslie Greenberg und seinen Mitarbeitern. Zum aktiven Ausdruck und zum Wiedererleben der Emotion wird die Technik mit dem Stuhl in der Gestaltarbeit genutzt. Der Wirkmechanismus dieser Technik liegt darin, dass die Probleme der Betroffenen mit dem Stuhl in der Gegenwart sichtbar, spürbar und erlebbar werden. In der Gestaltarbeit erlebt man, nicht nur die Handlung des Gegenübers zu erfassen, sondern den gesamten historischen Kontext, in welchem das emotionale Ergebnis steht, auszuleuchten (vgl. Frühmann 1994, S. 149), da die Technik mit dem Stuhl in der Gestaltarbeit nicht mit einfacher Beschreibung der Situation, sondern tiefenpsychologisch angewandt wird.

In der Arbeit mit dem "Stuhl“ werden beispielweise folgende Fragen von Therapeuten gestellt: „Wie alt bist du jetzt auf dem Stuhl?", „Wie alt ist das Gegenüber?“, „Wo bist Du? Was ist das für ein Ort?", „Was riechst du da?“, „Was fühlst du?", usw. In diesem Sinne kann man ihn „Erlebnisstuhl“ (Experiencing Chair) (Wagner-Moor 2004) nennen, da die Stuhltechnik während der Arbeit beim inneren Konflikt die Erfahrung und innere Exploration vertieft und bei dem unterbrochenen Prozess eine „expressive Stimme“ (expressive voice) (Clarke \& Greenberg 1988) möglich macht. Das spiegelt das gestalttherapeutische Axiom im Bezug auf experimentielle und existenzielle Behandlungsorientierung und „den unmittelbaren, phänomenologischen und gegenwartsgeleiteten Zugang“ (Hartmann-Kottek 2004) zum Problemfeld ganz effektiv wider, indem das Problem von Klienten durch sie selbst experimentell und erlebnisorientiert im Hier und Jetzt gezeigt und inszeniert wird.

Das kreative Medium ${ }^{10}$ „Stuhl“ wird als Projektionsmittel bezeichnet, mit dem ein

${ }^{10}$ Medien selbst haben keine kreative Fähigkeit sich zu entfalten. Identität der kreativen 


\section{Gestattherapie und Depression}

Aspekt des unintegrierten Teil des Selbsts, unterbrochende Prozesse oder bestimmte Personen sichtbar gemacht werden. Darüber hinaus zeigen sich durch sie die Beziehung zum Gegenstand und ihre Qualität. Dies hilft, dass der Klient sowie der Therapeut während der Vorstellung mit dieser Methode atmosphärisch und konkret das Problem verstehen, und dass die Emotion des Klienten noch deutlicher erweckt wird, indem die Rede in der Gegenwartsform erbeten wird. Dann hilft es dem Klienten weiter zum authentischen Ausdruck.

Hartmann-Kottek (2004) definiert die Zwei-Stuhl-Technik so, dass sie das Projektionsangebot für eine Konfliktsituation zwischen zwei annährend gleich starken, ambivalenten Strebungen (Impulsen, Tendenzen) ist, und von der Ein-Stuhl-Technik betont sie, dass wir eine Problemperson oder einen projizierten Aspekt als ein konkretes Gegenüber auf der Projektionsfläche des leeren Stuhls imaginär entstehen, mit diesem Gegenüber eine Begegnung erleben lassen und dabei eine Beziehungsklärung zur Veränderung der Beziehungsqualität einleiten (vgl. S. 214 u. 220).

Staemmler (1995) stellt die Methode mit dem Stuhl auf drei verschiedene Weise ohne Unterscheidung zwischen der Methode mit leerem Stuhl und der mit zwei Stühlen dar: (a) das Selbstgespräch mit dem Problem eines internen Konflikts oder von Polaritäten (b) die Bearbeitung von selbstunterbrochener Spaltung (c) das Phantasiegespräch mit dem Problem bezüglich unterbrochenen Prozesse mit einer bestimmten Person. Fragwürdig ist seiner Meinung nach das Selbstgespräch zwischen Topdog und Underdog, weil es sich als eine falsche Alternative und als unfruchtbar herausstellt, da sich „das Selbstgespräch zwischen Topdog und Underdog“ als Vermeidung entlarvt und nicht für alle Fällen geeignet ist. Staemmler schlägt stattdessen ein fruchtbares Selbstgespräch zwischen Bedürfnis und Vermeidung vor. Es lassen sich Vermeidung und Bedürfnis sowohl auf der Seite des Todogs, wie auf der des Underdogs finden. Greenberg, Rice und Elliot (1993; 2003) setzen das Selbstgespräch mit der Zwei-Stuhl -Technik immer dann ein, wenn ein Klient ein Muster von Selbstkritik oder Unterbrechung des Gefühlsausdrucks zeigt. Auf dem einem Stuhl sitzt dann derjenige, der sich z.B. selbst kritisiert, auf dem anderem Stuhl sitzt die Restpersönlichkeit, die an dieser Selbstkritik zu leiden hat. Diese Konstellation ähnelt sehr der Topdog-Underdog Situation von Perls, aber ist mit ihr nicht identisch, da die Pole in ihrer Grundhaltung und in ihrer Verständigungsbereitschaft nicht festgelegt sind. (vgl. Hartmann-Kottek 2004).

Medien ergibt sich im Kontext der Verwendung und Fähigkeit des Therapeuten zur Verwendung. Eine Erklärung folgt später. 


\section{Gestattherapie und Depression}

Dieses Beziehungsklärungsmodell wurde von den Autoren verfeinert (Greenberg et al. 2003, S.261) und als klinisch wirksam nachgewiesen. Die Methode mit dem Stuhl wurde in der Gestaltarbeit bei verschiedenen, konfliktbedingten Problemen verwandt. Die Effektivität der Arbeit wurde unter Verwendung der Stuhlmethode mit Variablen (Gewahrsein, Emotionale Aktivierung und Erfahrungstiefe) im klinischen Bereich, besonders bei der EFT, als erfolgreich nachgewiesen: Entscheidungsproblem (Greenberg \& Webster 1982), Konflikt oder Spaltung (Clarke \& Greenberg 1986; Greenberg 1979; Greenberg \& Clarke 1979; Greenberg \& Dompierre 1981; Greenberg \& Higgins 1980; Johnson \& Greenberg 1985), „unterbrochene Prozesse oder Gefühle“ (Greenberg \& Foerster 1996; Greenberg \& Malcolm 2002; Paivio \& Greenberg 1996), Depression (Beutler et al. 1986; Greenberg et al. 1998; Greenberg \& Watson 1999; Missirlian et al. 2005; Pos et al. 2003; Rosner 1996; Rosner et al. 1999; Tyson \& Range 1987), Misshandlung (Briere 1992) und Aggression (Conoley et al. 1983).

Die Technik mit dem Stuhl wurde in verschiedenen Bereichen mit anderen therapeutischen Ansätzen verglichen und als effektiv nachgewiesen. Die Ergebnisse der Studien berichten, dass die Methode mit dem leeren Stuhl im Vergleich zu psychoedukativen Gruppen bezüglich der Reduktion der Aggression (Beutler et al. 1986) und bei der Auflösung unterbrochener Prozesse (Paivio \& Greenberg 1995) effektiver ist; im Vergleich zur kognitiv-emotionalen Behandlung ist sie bezüglich der Reduktion der Aggression nahezu gleich wirksam. Die Ergebnisse der Studien, in denen die methodische Variante mit "Zwei-Stühlen" verwandt und mit anderen Ansätzen verglichen wurden, belegen, dass diese gestalttherapeutische Methode a) eine vertieftere Erfahrung und Veränderung des Gewahrseins mit sich bringt (Greenberg \& Clarke 1979; Greenberg et al. 1994; Greenberg \& Dompierre 1981; Greenberg \& Rice 1981) und b) nachweisbare Verhaltensveränderungen (Clarke \& Greenberg 1988) im Vergleich zur ausschließlich empathischen GesprächstherapieTechnik, und 3) deutlich intensivere Erfahrungen, ausgeprägtere Symptomreduktion und Veränderung des Gewahrseins (Clarke \& Greenberg 1988) im Vergleich mit der Technik von „Focussing plus Empathy“.

Die Ergebnisse der Studien (Clarke \& Greenberg 1986; Johnson \& Greenberg 1985), in denen mit der Methode "Zwei-Stühle" verwandt und mit kognitiv-behavioristischen Ansätzen bei Entscheidungsproblemen in der Ehe verglichen wurden, zeigen, dass die Methode und die Problem-Solving Technik aus dem kognitiv-behavioristischen Ansatz für die Reduktion der Unfähigkeit der Entscheidung gleich effektiv sind. Aber die Behandlung mit der Zwei-Stuhl-Technik erwies sich als deutlich wirksamer für die Anpassung und Feinabstimmung in der Ehe inclusive der Intimität (im Vergleich zum 


\section{Gestattherapie und Depression}

Zeitpunkt der achten Woche nach der Behandlung), als der kognitiv-behavioristische Ansatz (Johnson \& Greenberg 1985).

In der EFT wurde die oben beschriebene, gestaltherapeutische Methodik als Bestandteil der gesamten EFT-Behandlungsmethode integriert und als grundlegend und als immanent für die EFT charakterisiert und manualisiert, z.B. von Greenberg, Rice \& Elliot (1993). In diesem Manual werden Behandlungsaufgaben innerhalb der Therapie aufgezeigt, wobei Zeichen (Marker), Intervention und Endzustand beschrieben werden. Elliot (1999) teilt diese Aufgaben in vier Arten ein: (a) Grundlegende/ Aufmerksamkeitsorientierte Aufgaben, (b) Erlebnisorientierte Suche, (c) Aktiver Ausdruck und (d) Interpersonaler Kontakt. Bei dem "Aktiven Ausdruck" mit Stuhlmethoden wird bezüglich drei Aufgaben unterschieden, nämlich a) Spaltung im Hinblick auf die Selbstevaluation, b) selbstschädigende Spaltungen und c) unterbrochene Prozesse oder Gefühle.

Die Zwei-Stuhl-Methode wird sowohl verwandt bei der Spaltung im Hinblick auf die Selbstevaluation, also bei Selbstkritik oder Zerissenheit, mit dem Ziel der Selbstakzeptanz und Integration, als auch bei der selbstschädigenden oder unterbrechenden Spaltung (z.B. bei Gefühlsblockierung, Resignation) mit dem Ziel des Selbstausdrucks und zur Selbstbekräftigung. Bei den unterbrochenen Gefühlen wird die Methode mit dem leeren Stuhl mit dem Ziel der Akzeptanz gegenüber anderen, zur Selbststärkung und Loslösung verwandt (S. 342). Greenberg, Rice und Elliot (1993) verfeinern in ihrem Manual für die EFT Fälle, in denen die Zwei-Stuhl-Methode unterschiedlich genutzt wird. Falls man den Klienten dazu bringt, sich auf einen Dialog zwischen den widersprüchlichen Seiten seines Selbst einzulassen oder falls der Klient die Problematik selbstunterbrechender Spaltungen nach außen projiziert, sodass er sich seinen widersprüchlichen Seiten gegenüber machtlos, deprimiert und hoffnungslos fühlt. Der erste Fall ist Konfliktspaltung, der zweite selbstunterbrechende Spaltung.

In der Behandlung mit der "Zwei-Stuhl-Methode“ wurde das Prinzip dargestellt: der erste Schritt gilt der klaren Trennung von verschiedenen Aspekten des Klienten (Clearly separating out partial aspects of the client's self). Der zweite Schritt besteht in der Förderung der Aufmerksamkeit des Klienten und in der Ermutigung des emotionalen Erlebens und Ausdrückens (encouraging client attention to and heightening of emotional experiencing and expression). Der dritte Schritt ist der Beurteilung dieser Arbeit mit Zwei-Stühlen gewidmet, speziell der kognitiven und emotionalen Veränderungen des Klienten (assessing the two-chair work and cognitive and emotional changes with the client) (Daldrup et al., 1988). 
Beim ersten Fall gibt es verschiedene Formen von Spaltungskonflikten: Entscheidungskonflikte, Coachingskonflikte, Spaltung der Selbstbewertung und Attributionskonflikte. Hier geht es nicht darum, über einen Konflikt zu reden oder zu forschen, sondern es wird angestrebt, den Konfliktes in der Gegenwart zu durchleben, um dabei $\mathrm{zu}$ individuell passenden, neuartigen Lösungen $\mathrm{zu}$ gelangen. Das grundlegende Problem besteht hier darin, die Sollensforderungen und die eigenen Bedürfnisse in Einklang zu bringen, die negative Bewertung des eigenen Wollens in ein Akzeptieren umzuwandeln und die eigenen Bedürfnisse auf eine Weise zu befriedigen (Greenberg et al. 1993, S. 259). Von den Autoren wurde ein Modell des Auflösungsprozesses von Konfliktspaltungen Entwickelt. Es hat drei Hauptphasen: die des Gegensatzes bzw. der Opposition, die der Identifikation und des Kontakts und schließlich die der Integration. Das Modell ist abgeleitet von den gestalttherapeutischen Konzepten des „Topdog und Underdog“. Zur Beurteilung des erreichten Auflšsungsgrades wurde eine Skala in Kurzform entworfen (a.a.O. S. 261):

(a) Beschreibung einer Spaltung, bei der ein Aspekt des Selbst einen anderen nicht akzeptiert oder ihn in einen Zwang zu unterwerfen versucht.

(b) Kritik, Erwartungen oder Sollensforderungen werden dem Selbst gegenüber klarund auf konkrete und spezifische Weise zum Ausdruck gebracht.

(c) Die primären, grundlegenden Gefühle des Klienten in Reaktion auf die Kritik tauchen auf.

(d) Bedürfnisse und Wünsche, die mit einem neu erlebten Gefühl verbunden sind, werden klar zum Ausdruck gebracht.

(e) Der Klient akzeptiert aufrichtig seine Gefühle und Bedürfnisse. Möglicherweise werden auch Mitgefühl, Fürsorglichkeit und Respekt gegenüber dem Selbst gezeigt.

(f) Ein klares Verständnis dessen, wie unterschiedliche Gefühle, Bedürfnisse und Wünsche berücksichtigt werden können und wie zuvor widerstreitende Seiten des Selbst in einer funktionsfähigen Beziehung miteinander versöhnt werden können, ist entstanden.

Bei der Intervention des zweiten Falls hilft der Therapeut dem Klienten, bewusst zu merken, dass er sein Muster der Unterbrechung oder Blockierung des Selbstausdrucks verursacht hat und unterstützt, die unterbrochenen Emotionen zum Ausdruck zu bringen. Dabei gewinnt der Klient die Fähigkeit sich auszudrücken und frei mit seiner Umgebung in Kontakt zu treten zurück. Wichtig ist in diesem Fall, dass der Klient seiner Gefühle gewahr wird und daaa er sich dabei gestärkt fühlt, da Selbstunterbrechung mit einem automatisch aktivierten emotionalen Schema 
verbunden ist, während die Emotionen ständig oder stark blockiert wurden und folglich Zweifel, Hilflosigkeit oder Hoffnungslosigkeit statt Selbstwertgefühl entstehen. Das Modell des Auflösungsprozesses von selbstunterbrechender Spaltung wurde in drei Hauptphasen dargestellt: die der Darstellung bzw. Inszenierung, die des Erkennens der eigenen Urheberschaft und schließlich die des Kontakts. Dabei wurde die Kurzform einer Skala zur Beurteilung des erreichten Auflösungsgrades wie folgt (a.a.O. S. 297) entworfen:

(a) Der Klient wirkt auf das Selbst so ein, dass sein Ausdruck unterbrochen wird, oder er beschreibt, wie ein Teil von ihm den Ausdruck eines anderen Teils unterbricht.

(b) Der Klient betätigt sich auf konkrete und spezifische Weise aktiv als Verursacher des Selbstunterbrechungsprozesses.

(c) Die Passivitäts- und Resignationsgefühle des Klienten werden kontaktiert und differenziert.

(d) Der Klient bringt die unterbrochene Emotion klar zum Ausdruck.

(e) Das mit der Emotion verbundene Bedürfnis wird vom Klienten klar ausgedrückt.

(f) Der Klient fühlt sich wieder im Besitz seiner Macht und fängt an, neue Aktivität ins Auge zu fassen oder zu planen, um auf die Erfüllung seiner Bedürfnisse hinzuarbeiten.

Falls der Klient ein Problem bezüglich einer bestimmten Person oder wichtigen Bezugsperson oder eines unterbrochenen Prozesses oder entsprechender Gefühle hat, wird die Ein-Stuhl-Methode genutzt. Die Arbeit mit dem leeren Stuhl wurde ausführlich bei der Arbeit mit Personen, die in ihrer Kindheit missbraucht oder misshandelt wurden, genutzt (Briere 1992). Diese Arbeit mit dem leeren Stuhl zielt darauf $\mathrm{ab}$, vermeintlich negative Gefühle auf relevante Personen (meist die Eltern) zu lenken (Elliot 1999, S. 345). Fünf Funktionen dieser Methode sind von Greenberg (1979) herausgestellt worden: (a) Trennung und Schaffen der Kontaktstelle (Separation and the creation of contact) (b) die Verantwortung des Klienten (the responsibility of the client) (c) die Teilnahme (the attending) (d) die Steigerung (the heightening) (e) die Expression (the expressing). Der Arbeitsprozess führt zur

„Auflösung früherer Probleme der Beziehung oder zum Abschluss traumatisierter Erlebnisse durch Wiedererleben, aktiven Ausdruck bisher nicht ausgedrückter Gefühle und Akzeptieren unerfüllter Bedürfnisse. Diese Arbeit mit dem leerem Stuhl hilft dem Klienten, neuer Informationen und Bedeutungen gewahr zu werden, die auf alten, emotionalen Erlebnensweisen beruhen, und seine Aufmerksamkeit darauf zu richten“ (Greenberg et al. 2003, 
S. 322- 323).

Hierzu wurde die folgende Kurzform einer Skala zur Beurteilung des erreichten Auflösungsgrades entworfen (a.a.O. S. 327):

(a) Klient beschuldigt, klagt oder bringt Verletztheit oder Sehnsucht hinsichtlich einer wichtigen Bezugspersonen zum Ausdruck.

(b) Klient tritt zum Anderen in Kontakt und drückt unaufgelöste Gefühle, oft Groll oder Verletztheit, aus.

(c) Durch Differenzierung der Beschwerde werden die ihr zugrunde liegenden Gefühle deutlich. Außerdem werden die relevanten Emotionen erlebt, im allgemeinen Traurigkeit und Wut, und mit starker emotionaler Erregung zum Ausdruck gebracht.

(d) Der Klient empfindet sein unerfülltes Bedürfnis als berechtigt und bringt es selbstbewusst zum Ausdruck.

(e) Der Klient lernt, den Anderen auf neuartige Weise zu sehen und zu verstehen. Entweder sieht er ihn in einem anderen Licht oder als einen weniger machtvollen Menschen, der selbst Probleme hat.

(f) Der Klient bejaht sich selbst und löst sich von dem unaufgelösten Gefühl, indem er dem Anderen entweder vergibt oder ihn zur Verantwortung zieht.

Greenberg und Watson (1999) stellen vergleichende Ergebnisse von zwei Behandlungen bei Depressionen vor : die eine arbeitete mit der empathischen klientzentrierten Behandlung und die andere mit der gestaltorientierten Behandlung. Die gestaltorientierte Behandlung umfasste drei Aufgaben entsprechend den spezifischen Indikatoren für Klienten und den zugehörigen, therapeutischen Interventionen nach dem Manual des EFT. Die ersten drei Sitzungen wurden in rein kontaktorientierter, empathischer Weise durchgeführt. Nach der dritten Sitzung wurde je nach Bedarf der Zwei-Stühle-Dialog und der Dialog mit dem leeren Stuhl einschließlich „Focussing“ (Gendlin 1996) verwandt. „Diese Behandlung konzentriert sich auf den Zugang zu primären adaptiven Gefühlen und auf die Aktivierung unterschiedlicher Arten dysfunktionaler emotionaler Schemata, um diese neu zu strukturieren“ (Greenberg et al 1993). Die Lösungsprozesse jedes dieser Probleme wurden im Einzelnen modellmäßig, wie oben beschrieben, dargestellt.

Bei depressiven Klienten gibt es häfig Hinweise auf „unterbrochene Prozesse oder Gefüle"(unfinished Business), insbesondere wenn die Depression mit Verlusten in Zusammenhang steht. Depressive Klienten leiden häfig an einer Aufspaltung der emotionalen Verarbeitung in eher emotional- erlebnisorientierte und andererseits in 
intellektuelle bzw. distanzierende Aspekte. Diese Brüche in der Verarbeitung führen zu emotionalen Blockaden oder Lähmungen, oftmals in Form sekundärer, reaktiver Emotionen. Sie werden am deutlichsten, wenn sich Klienten während der Sitzung beim Ausdruck dessen blockieren, was sie gerade fühlen, und statt des Ausdrucks nonverbal etwas zeigen, z.B. kšrperliche Beschwerden, mimische Zeichen der Unterdrückung. Dies ist besonders typisch für die klinische Form der Depression (Elliot 1999).

Trotz der Effektivität der Behandlung mit der Verwendung der "Stuhlmethode“ muss diese Methode vorsichtig angewandt werden, da sie in hohem Maße emotional überfluten und belasten kann. Deshalb ist es sinnvoll, dass man den Klienten in seinem Tempo arbeiten lässt (Hartmann-Kottek 2004) und notfalls vor sich selbst schätzt. Man kann den Klienten im Falle einer zu ausgeprägten, emotionalen Erregung statt in die Identifikation mit seinen emotional aufgeladenen Polen lieber anregen, die Methode der Bedeutungsbildung zu wählen. Dabei lässt man den Klienten zunächst unerträgliche emotionale Zustände symbolisieren, wodurch etwas innere Distanz entsteht. (Elliot 1999). Darüber hinaus muss man bei der Anwendung dieser Methode darauf achten, ob Klienten tatsächlich genug emotional vorbereitet sind, diesen Schritt $\mathrm{zu}$ machen. Sonst fühlt sich der Klient in der Situation überfordert und das macht keinen Sinn.

Deshalb muss die Voraussetzung erfüllt sein, dass sich erstens die Emotion der Klienten für die Arbeit ausreichend abzeichnet, wie das im Manual für die EFT konzipiert und beschrieben wurde. Zweitens: wenn sich der Klient emotional genügend vorbereitet fühlt, dann klärt der Therapeut seinen Klienten unmittelbar vor der Anwendung über die Methode und ihre wahrscheinliche Wirkung auf. Diese Methode ist für Menschen mit Selbstunsicherheit, erhöhtem Angstpotenzial, Rückzugsund überanpassungsbereitschaft besonders gut geeignet (vgl. Hartmann-Kottek 2004). Es besteht eine allgemeine Indikation zur Verwendung dieser Technik, da diese Technik immer von der dialogischen Grundhaltung geprägt ist. Diese Technik ist nur ein „Katalysator“ (Oaklander 1979). Ihre spezifische Ausprägung hängt immer von dem Kontext ihrer Verwendung und der therapeutischen Beziehung ab.

Für die Indikation oder Kontraindikation zur Nutzung der Methode mit dem Stuhl gelten schon aus den frühen Zeiten der Gestalttherapie die Hinweise einer Studie von Fagan et al., 1974. Die Autoren schlagen folgendes vor: Der Therapeut sollte a) diese Technik nicht verwenden, sofern er nicht schon selbst persšnliche Erfahrung mit ihr gehabt hat, b) zu den Explosionen und zu den starken emotionalen Reaktionen bereit sein und c) seinen Patienten gut genug kennen, um Follow-up-Unterstützung geben zu können, denn ungelöste innere Konflikte können für zerbrechliche Patienten 


\section{Gestattherapie und Depression}

zerstörend sein. Diese Technik kann für die schwer geschädigte Patienten bei Trauma, schweren kognitiven Störungen, Problemen der Impulskontrolle, schwere Persönlichkeitsstörungen, Psychose (Nevis \& Melsnick 1996; Saltzman 1989), ichstrukturellen Störungen und für Personen, die aktuell Kriseninterventionen brauchen (vgl. Elliot \& Greenberg 1995) kontraindikativ sein, denn sie führt in eine starke, emotionale Involvierung, die einen chaotischen Prozess auslösen kann. Zusammenfassend lässt sich feststellen, dass man keine konfliktaufdeckende Konfrontation bei unausgereifter oder destabilisierter Ichstruktur durchführen sollte.

Frühmann (1994) schlägt als Kriterien der Kontraindikation Folgendes vor: die konfrontative Methodik sollte nicht angewandt werden, erstens in Bereichen, wo der Klienten auf dem Hintergrund seiner Befindlichkeit zu schützen ist, z.B. bei Klienten mit Antriebslosigkeit sowie Motivationsschwierigkeiten, mit hoher Involvierung, hohem Angstpegel oder Starre (paranoide Qualität) und bei Inhalten, deren Identifikationspotential gefährdend oder diskriminierend wirken könnten, - und zweitens in Fällen, wo das Instrumentarium selbst Artefakte setzt, z.B. bei der Gefahr von Leiterfixierung, der Inkompatibilität von Zielsetzung und Methode und der Inhalte, die als Gruppenthemen zu sehen sind (vgl. S. 150-152).

Ein Grundprinzip muss in der Arbeit mit Gefühlen bei Depression vorausgesetzt werden: dem Klienten erklären, warum es vorteilhaft ist, depressive Emotion z.B. Hilflosigkeit oder Traurigkeit in seiner Tiefe $\mathrm{zu}$ erfahren. Beim Erfahren von Hilflosigkeit, die sowohl mit Gefühlen von Traurigkeit, Ärger, Furcht und einem dysfunktionalen Glauben zu sich und zum anderen einhergeht, als auch mit unerfüllten Wünschen und Bedürfnissen, begegnet man der Angst vor verdrängten oder vernachlässigten Bedürfnissen. Wenn der Klient besser verstanden hat, was seine Hoffnungslosigkeit erzeugt hat, arbeitet er dann überzeugender in der Therapie mit, um herauszufinden, was seine Bedürfnisse sind und waren und wie seine Ressourcen zur Erfüllung seiner Bedürfnisse verfügbar werden können, um eine Veränderung oder eine Restrukturierung der Gefühle zu bewirken.

\subsubsection{Ergebnisse der Behandlung von Depressionen}

Tyson und Range (1987) untersuchten 44 sich selbst depressiv empfindende Studenten, die nach der Subskala 2 (Depression) des MMPIs sortiert wurden. Sie wurden in vier Gruppen randomisiert und in vier Sitzungen einmal in der Woche behandelt. Dabei wurde auch auf die Vergleichbarkeit der vier Therapieformen geachtet. Als Therapiegruppen fungieren: 1) die Kontrollgruppe, 2) die Gestaltgruppe mit dem 


\section{Gestattherapie und Depression}

Einsatz des leeren Stuhls, in der dieBehandlung Topdog-Underdog durchgeführt wurde, 3) die Dialoggruppe mit dem Einsatz einer Szene von Shakespeare, in der Teilnehmer neutral ihre Gefühle ausdrücken und 4) eine Expressionsgruppe, in der die Gefühle der Teilnehmer zweimal pro Sitzung einfach stark ausgedrückt werden. Die Ergebnisse zeigen, dass die Gestaltgruppe mit der Verwendung des leeren Stuhls empirisch am effektivsten ist, dass der Effekt nach zwei Monaten weiter nachweisbar ist, und auch zur Reduktion der Angstsymptome beigetragen hat. Obwohl die Klienten in dieser Studie nur vier Stunden behandelt wurden, zeigten die Ergebnisse, dass der Dialog mit dem leeren Stuhl zur Reduktion depressiver Symptome effektiv ist und ein dauerhafter Effektbesteht.

Die Studie von Greenberg und Watson (1998) enthält vergleichbare Ergebnisse.Eine Prozessstudie mit 34 Klienten, die an einer starken depressiven Störung leiden und denen 16-20 Einzelsitzungen einmal in der Woche angeboten wurden, lief mit dem Vergleich der gestaltorientierten und der klientzentrierten Therapieform. Die Ergebnisse zeigen, dass die gestaltorientierte Behandlung für die Reduktion depressiver Symptome effektiv war, und dass der Dialog zwischen zwei Stühlen und der Dialog mit dem leeren Stuhl dazu führten, dass die Klienten einen größeren Zugang zu ihren inneren Erfahrungen bekommen hatten und die Lösung spezifischer emotionaler Probleme gefunden wurde. Aus dieser Studie wurden zwei Fälle, zu denen ein erfolgreicher und ein erfolgloser gehörte, zur Untersuchung für die Assimilation problematischer Erfahrungen ausgewählt (Honos-Webb et al. 1998). Bei dem erfolgreichen Fall fand ein Assimilationsprozess statt, aber bei dem erfolglosen Fall war er blockiert.

In der Studie (Rosner et al. 1999) wurden die BDI-Verläufe von 63 biopolar depressiven Patienten mit Hilfe Hierarchischer Linearer Modelle (HLM) untersucht. Die Patienten hatten randomisiert an einer von drei ambulant durchgeführten Psychotherapieformen, Kognitive Verhaltenstherapie, EFT und unterstützende selbstadministrierte Bibliotherapie, mit je 20 wöchentlichen Sitzungen teilgenommen. Dabei gab es leider keine Information darüber, ob die Behandlung im Gruppen- oder Einzelsetting durchgeführt worden war. An der Bibliotherapie hatten die Patienten per Telefon jeweils 30 Minuten teilgenommen. Die Ergebnisse zeigten, dass schwer depressive Patienten eine schnellere Verbesserung als weniger depressive Patienten zeigten, und dass die Wirksamkeit beider Behandlung EFTs bzw. der Verhaltenstherapie signifikant effktiv war.

Die Studie Missirlian et al., 2005 wurde bei 32 depressiven Klienten durchgeführt, mit der Zielsetzung, „emotional Arousal, perceptual Processing and Working Alliance“ zu 
unterschiedlichen Zeitpunkten zu untersuchen: am Anfang, in der Mitte und am Ende des Therapieverlaufs. Bei Behandlungen, die nach dem Manual der EFTs konzipiert wurden, wurden den Klienten 14-20 Einzelsitzungen einmal in der Woche angeboten. Die Ergebnisse zeigten, dass hohe emotionale Erregung (emotional Arousal) sowohl für den Prozess des Klienten als auch für die therapeutische Effektivität unabdingbar ist und einen wichtigen Prädikator zur Reduktion depressiver und psychopathologischer Symptome sowie zur Erhöhung des Selbstwertgefühls, besonders in der Mitte der Therapie, bedeutet. Die hohe Intensität der Wahrnehmungsentwicklung (perceptual processing) ist die einzige Variable, die zur Veränderung am Anfang der Therapie führt und ein Prädikator für die Effektivität der Therapie am Ende ist. Das Arbeitsbündnis (Working Alliance) beeinflusst die Reduktion des depressiven Symptoms nicht.

In der Studie (Pos et al. 2003) wurde der emotionale Prozess, der mit dem „Experiencing Scale“ (EXP, Kein et als. 1986) erhoben wurde, mit dem Vergleich am Anfang und am Ende der Therapie zur Veränderung der depressiven und allgemeinen Symptome, des Selbstwertgefühls und der interpersonalen Probleme untersucht. In der Gruppe, die nach EFT behandelt wurde, hatten 34 Klienten die Diagnose der Major Depression unterden Kriterien DSM-IV erfüllt; sie erhielten 16-20 Einzelsitzungen einmal in der Woch. Der emotionale Prozess ist ein Prädiktor 1) zur Reduktion der depressiven und psychopathologischen Symptome und 2) zur Erhöhung des Selbstwertgefühls zu den Zeitpunkten vor und nach der Therapie.

\subsection{Berücksichtigung der gestaltorientierten Behandlung von Jugendlichen bei Depression}

Bei der Entwicklung, sowohl der angemessenen Diagnostik als auch der Methodik für die spezifischen psychischen Probleme, trägt die Psychotherapie heutzutage noch Kinderschuhe. Das Verständnis der Entwicklung und das Wissen um ein altersgemäßes Entwicklungsgeschehen bestimmen in der psychotherapeutischen Arbeit mit Jugendlichen im wesentlichen Diagnostik und Methodik. Auffälligkeiten und psychische Beeinträchtigungen eines Jugendlichen weisen immer auf eine prävalent pathogene Umwelt hin, die das Wachstum und die Entwicklung eines Jugendlichen behindert und eine kreative und expressive Persönlichkeit unmöglich machen kann. Wie bei Erwachsenen muss eine therapeutische Arbeit bei Jugendlichen dazu beitragen, einen sicheren Ort anzubieten, wo man sowohl einen Zugang zu seinem beeinträchtigten Teil finden, sich verstanden fühlen und seine 


\section{Gestattherapie und Depression}

Potentiale und Ressourcen entfalten kann, indem man akzeptiert wird. Wie kann man diesen für Jugendliche angemessenen Ort schaffen? Dies ist die Frage für Therapeuten, Berater und Sozialarbeiter, die mit Jugendlichen zu tun haben. Lediglich in den Methoden finden einige Therapeuten die Anwendungsmöglichkeiten für Kinder und Jugendliche.

Das Spiel und der Einsatz von kreativen Medien sind von besonderer Bedeutung, da sie Wirkungen auf verschiedenen Ebenen ${ }^{11}$ ermöglichen, einen sicheren Ort $\mathrm{zu}$ schaffen. Die erste Ebene ist der Zugang zum eigenen Land. Diese Ebene gilt nicht nur für Klienten selbst sondern auch für Therapeuten, wie man seine Augen mit denen eines Kindes gleich kontaktiert, wenn man ein Kind anspricht. Manche auffälligen oder psychisch beeinträchtigten Jugendliche mšgen nicht oder sind unfähig, über sich zu erzählen. Therapeuten scheitern manchmal deshalb mit Ihnen zu kommunizieren. Und manche belastende Dinge sind ganz schwer für Jugendliche ausschließlich zu verbalisieren. Der Einsatz von kreativen Medien ermöglicht es, die verbal unzugängliche Ebene zu erreichen.

Die zweite ist die Ausdrucksebene. Jugendliche sind noch unreif, ihre Emotionen konkret zum Ausdruck zu bringen. Insbesondere sind depressive Jugendliche im Ausdruck gehemmt. Beide Ebenen sind unabdingbar für die Entwicklung einer therapeutischen Beziehung. In der Gestalttherapie hat Violet Oaklander (1988; 1994a; 1994b) die Möglichkeit der Behandlung von Kindern und Jugendlichen in der Anwendung verschiedener Medien gefunden. Sie hat desweiteren den Ansatz der "Gestalt Play Therapy“(vgl. Blom 2006), die gestalttherapeutische Prinzipien und Techniken für die Arbeit mit Kindern und Jugendlichen beinhaltet, entwickelt, wobei verschiedene Formen und Techniken des Spiels in differenzierten Phasen genutzt werden, um Kindern und Jugendlichen die Chance zu geben, sich verbal sowie nonverbal auszudrücken und zu entfalten. Oaklander (1994a; 1994b) beschreibt als die theoretischen Grundlagen der "Gestaltspieltherapie“ Beziehung, Selbstregulation, Kontaktstörung, Gewahrsein, Experience und Widerstand. Die oben erwähnten Studien im Kapitel. 2.2.2. zeigten die Effektivität der gestaltorientierten Behandlungen bei Depression. Aber in den Studien wurden erwachsene Klienten untersucht. Trotz der Überzeugung der Effektivität der gestaltorientierten Behandlungen bleibt es noch fraglich, ob die Behandlungsmethoden für Jugendlichen auch geeignet sind, wenn man sie umsetzt.

Besonders ist die "Stuhlmethode“ trotz ihres Effekts bei der Anwendung von

${ }^{11}$ Auf die Wirkungsebene und Anwendungsmöglichekeiten kreativer Medien werde ich später anschaulich eingehen. 
Depression schwierig bei Jugendlichen anzuwenden. Denn die Arbeit verlangt mit der Methode hohe Konzentrationsfähigkeit vom Klienten auf den Gegenstand, nämlich dass der Klient auf dem Stuhl inszeniert oder phantasiert. Bei der Arbeit mit dem leeren Stuhl phantasiert der Klient seine Bezugsperson, auf die sich seine „unterbrochenen Prozesse oder Gefühle" beziehen, mit Hinblick auf den Stuhl. Schlimm kann dabei werden, wenn er sein Gefühl schnell verliert. Bei der Arbeit mit zwei Stühlen projiziert der Klient seine gegenseitigen Teile, an deren Konflikt der Klient leidet, auf die Stühle. Der Klient kann unfähig sein, seine Pole oder unintegrierten zwei Seiten, also Konfliktsituationen nicht konkret verschränkt zu projizieren, so dass er sich in der Arbeit nicht emotional erlebt sondern abstrakt.

Für die Behandlung von Jugendlichen zeigt die Arbeit mit dem Einsatz kreativer Medien einen guten Weg auf. Für die lebendige Exploration und Belebung auf verschiedenen Ebenen in der Anfangsphase und zum aktiven Ausdruck in der Mitte der Gestaltarbeit habe ich die Möglichkeit des Einsatzes kreativer Medien für einen fokalen und effektiven Weg gefunden. Als therapeutische Grundlage wird in dieser Arbeit die Gestalttherapie verwendet. Das oben untersuchte Behandlungsmodell von Depression in der EFT ist zur Auflösung des Konfliktes und der Spaltung bei aktivem Ausdruck hier gut geeignet, besonders sein gesamter Therapie-Verlauf, z.B., was in der Anfangs-, Mitte- und Endphase exploriert wird. Methodologisch wird diese Arbeit kreativen Medien verwenden. Im folgenden Kapitel wird die Möglichkeit der Verwendung von kreativen Medien bei der Depressionsbehandlung von Jugendlichen weiter erklärt. 


\section{Einsatz kreativer Medien in der Gestalttherapie}

\subsection{Zum Begriff der "kreativen Medien“}

Menschen brauchen Medien für Kontakt, Kommunikation und Ausdruck mit sich selbst und mit anderen. Die Medien sind so alt wie die menschliche Geschichte. Sie verändern und ergänzen sich miteinander bei der Entwicklung der Gesellschaft und bei der individuellen Entwicklung. Ein Mensch nutzt Körpersprache als Medium, dann Lautemachen, Sprache, Malen, und entwickelt seine Kommunikationsweise von Mimik und Gestik bis zur Technologisierung. Menschen beschäftigen sich damit, wie sie sich für sich und für andere in verschiedenen Situationen effektiv ausdrücken können, wie sie mit neuen Medien umgehen können. In der Psychotherapie spielt seit der Psychoanalyse Freuds Sprache eine Hauptrolle. Indem sich die verbal orientierte Therapie auf Bedeutung und Zusammenhang des seelischen Problems mittels Sprache fokussiert, eröffnet sich eine neue Perspektive auf die Beziehung Körper-Geist-Seele in den verschiedenen Schulen und Richtungen der Psychotherapie und -beratung. z. B. den Dualismus bahnbrechender Arbeit Wilhelm Reichs, Alexander Lowens und Fritz Perls`. Sie berücksichtigen nicht nur die Bedeutung der Körperkommunikation, sondern entwickelten auch Körpertherapieverfahren, z.B. Tanz-, Atem- und Bewegungstherapie. In diesem Sinn ist Körper und Leib als Medium eine wichtige Informationsquelle und ein Zugang zur inneren Welt des Klienten.

Die Psychotherapie nutzt Kunst in verschiedener Art und Weise und mit unterschiedlichen Zielsetzungen. Dabei kommen verschiedene künstlerische Medien (bildnerische, literarische, musikalische), Imagination, Rollenspiel und Märchen etc. zum Einsatz. Diese neue Arbeitsweise führte einerseits zur Entstehung der eigenständigen Therapieform der Kunsttherapie, und andererseits $\mathrm{zu}$ einer Integration ihrer Methoden in bestehende Therapieverfahren. Für die Kunsttherapie besteht das Problem, sich von diesen integrativen Arbeitsweisen abzugrenzen. Für die Kunsttherapie allein scheint es schwer, eine theoretische Anknüpfung an die Praxis zu finden, denn sie selbst erfüllt nicht ausreichend den Rahmen der Therapie als „wissenschaftlich begründete, zielgerichtete klinische Arbeit mit einem Klienten auf der Grundlage einer Diagnose, einer spezifischen Indikationsstellung, einer Theorie der Persönlichkeit, der Darstellung der anthropologischen Menschenbilder" (Petzold \& Sieper 1990, S. 170), sondern ergänzt ihre Schwäche in der bestehenden therapeutischen Theorie und Praxeologie. An jedem Zweig verschiedener 


\section{Einsatz kreativer Medien in der Gestalttherapie}

Therapieschulen entstanden deshalb neue Therapieformen, die kreative Medien und künstlerische Methoden in die therapeutische Behandlung einbezogen haben. z.B. psychoanalytisch (Dreyfuss-Kattan 1986), Person-zentrierte-expressiv (Rogers 2001) und Kognitive-Behavioral orientiert (Rosal 2001) bzw, Jungianisch analytisch (Edwards 2001), Gestalttherapeutisch (Rhyne 1973/1996), Kreativitätstherapeutisch (Petzold \& Orth 1990) ausgerichtet. Im Bereich der Musik-, Körper-, Tanz-, Poesietherapie etc. ist es möglich diese verschiedenen Kombinationen zu schaffen. Aber Kunsttherapie steckt bei der Entwicklung „,zum Allein-Stehen“ noch in den Kinderschuhen.

Die Arbeit mit künstlerischen Materialien oder kreativen Medien, in der man Gemeinsamkeiten zwischen künstlerischer Aktivität und therapeutischem Prozess exploriert, die Potentiale zur Heilung aus künstlerischer Aktivität und die Therapiemethoden unterschiedlicher klinischer Bereichen einbezieht und anwendet, wird einerseits insbesondere für die Behandlung von Kindern und für die Diagnostik eingesetzt und andererseits von verbal orientierten Therapeuten als ketzerische Haltung kritisiert, weil sie die Kunst geplündert hat, um aus ihr Therapietechniken zu gewinnen (vgl. Miller 2006).

Der Versuch einer Integratgion könnte nach dem Integrationsparadigma von Petzold (2003a) als Methodenintegration gesehen werden. Will man den Versuch zur Integration nicht scheitern lassen, muss man seine Aktivitäten darauf ausrichten, für welche Ziele ein solcher Versuch unternommen werden soll. Das bedeutet, dass das Ziel, wofür eine Methode in ein Therapieverfahren einbezogen werden soll, eindeutig etabliert werden muss, indem die spezifischen Wirkfaktoren sowohl aus den grundorientierten Therapieverfahren als auch aus den Methoden und Medien herauskristallisiert werden. „Nicht die Methoden werden die Interventionspraxis bestimmen, sondern die Ziele“ (Petzold, Leuenberger \& Steffan 1998) und "die dahinter stehenden Probleme, Ressourcen und Potentiale" (Petzold 2003a). Werden zunächst also Charakter der Verfahren und Merkmale der Methoden und Medien differenziert, können diese Methoden und Medien die Chance zur Integration oder zur kreativen Anwendung in einem Therapie- oder Beratungsverfahren haben.

In diesem Freiraum kann die Identität kreativer Methoden und Medien entwickelt werden. Die Rolle einer Methode oder eines Mediums verändert sich in jedem Kontext, je nachdem wofür und in welchem Rahmen sie genutzt wird. Ein Medium selbst ist nur ein Informationsträger. Aber es ist abhängig von dem Kontext und der Fähigkeit des Benutzers, seine Potentiale zu entdecken, zu entfalten und sie kreativ anzuwenden.

Hartmann-Kottek als Gestalttherapeutin betont, dass "sich der Aufforderungscharakter der Medien zwar ein wenig unterscheidet, und das ist reizvoll, 


\section{Einsatz kreativer Medien in der Gestalttherapie}

aber die Medien werden in ihrem Rahmen nie ganz Selbstzweck, sondern dienen stets der Selbstbegegnung“ (2004, S. 231).

Petzold (2003) unterscheidet Methode, Technik und Medien und erklärt ihre Beziehung: „Methode als konsistentes, theoriegeleitetes Ensemble von Strategien im Rahmen eines Verfahrens, das über Techniken und Medien verfügt, steht im Dienste der Realisierung von Zielsetzungen, Zielen, die durch die Erfordernisse der Lebenssituation und theoretischen Konzepte des Verfahrens vorgegeben sind. Techniken sind Instrumente zur Strukturierung von Situationen im Rahmen einer Methode. Medien sind Träger von Informationen und Gegenstand der Aktion“ (Bd. 2, S. 507). Medien werden in verschiedener Weise in der Therapie eingesetzt. Ihre Identität und Bedeutung in der Therapie entstehen immer je nach theoretischem Hintergrund, therapeutischer Beziehung, ihren Beitragsweisen und ihrem Beitragsausmaß zur Veränderung des Klienten. Identität und Bedeutung der Medien in der Therapie sind jedenfalls nicht vom Kontext, in dem Technik und Methode angewandt werden, zu trennen. Was ich im Folgenden als Funktionen oder Potentiale kreativer Medien nennen will, soll immer im therapeutischen Rahmen von Gestaltarbeit, verstanden werden. Deshalb muss die Bedeutung der „Identität und Wirkpotentiale kreativer Medien" im Kontext des jeweiligen Arbeitsansatzes, hier in Bezug auf die gestalttherapeutische Arbeit, verstanden werden.

An dieser Stelle müssen noch zwei Begrifflichkeiten geklärt werden. Zum einen, warum in der vorliegenden Arbeit nicht der Begriff der "gestaltorientierten Kunsttherapie“ oder der "Gestaltarbeit mit künstlerischen Medien" verwendet wird, sondern es um "Gestaltarbeit mit kreativen Medien“ geht, zum anderen, warum es ausdrücklich um „kreative“ Medien geht. Einerseits werden in der vorliegenden Arbeit im gestalttherapeutischen Setting kreative Medien aus der Kunsttherapie verwenden. Unter Kunst versteht man im deutschen Sprachraum eher die Zentrierung auf den Bereich des Bildnerischen. "Sicherlich kann hier nicht auf die "große Kunst" abgestellt werden. Diese ist Ergebnis eines künstlerischen Schaffensprozesses. „Zur Rede steht, sowohl beim Begriff Psychotherapie wie bei dem der Kunsttherapie, die Verwendung künstlerischer Methoden, Techniken und Medien zur Stimulierung von expressiven Fähigkeiten, zur Schärfung des Wahrnehmungsvermögens im Rahmen therapeutischen Handelns, wie immer dies auch orientiert und geartet sein mag“(Petzold \& Sieper 1990, S.176). Andererseits kann der Begriff „künstlerisch“ als problematisch ausgesehen werden. In moderner Kunst ist es schwierig abzugrenzen, was „künstlerisch“ ist. Alle Materialien können künstlerisch genutzt werden. Im 


\section{Einsatz kreativer Medien in der Gestalttherapie}

therapeutischen Prozess wird etwas Gestaltetes vom Klienten kreiert, das als Kunst bezeichnet werden kann, aber keinesfalls die Gestaltung eines Kunstwerkes zum Zweck hat. In diesem Sinne sind die Medien kreative Einsatzmöglichkeiten, die in jedem Kontext sowohl vom Therapeuten oder Berater als auch vom Klienten zum Ausdruck ihrer kreativen Potentiale genutzt werden können, ohne sie künstlerisch abgegrenzt zu haben. Dann werden Medien von der hierarchischen Macht der Kunst frei, sind für ihre Benutzer zum aktiven Engagement verfügbar.

Aus diesen Gründen verwende ich in der vorliegenden Arbeit den Begriff der "Arbeit mit kreativen Medien", sodass Therapeuten und Klienten den Freiraum kreativer Medien nutzen und erweitern können. Steine, Blätter, Tücher und Teddybären werden auch zu kreativen Medien, nicht nur Malmaterial, Musik, Theater und Drama oder Literatur. Das bedeutet, dass alle Materialien zur Entdeckung und Entfaltung ihrer Potentiale zur Verfügung stehen, wie z.B. Perls mit dem Stuhl seine berühmteste Methode "der leere Stuhl“ in der Gestalttherapie kreativ entwickelt hat. Medien sind dann „kreative Medien“, wenn „durch ihr stimulierendes Potential der Selbstausdruck gefördert wird, sich dadurch neue Handlungsspielräume eröffnen und wenn sie im kreativen Gestaltungsprozess dazu herausfordern, unbewusste psychische Inhalte nach außen in die Bewusstheit (awareness) und ins Bewusstsein (conciousness) dringen $\mathrm{zu}$ lassen“ (Höhmann-Kost 2003). Schließlich müssen „kreative Medien" immer im therapeutischen Kontext verstanden werden, sodass die Identität kreativer Medien in einer therapeutischen Beziehung sich an der therapeutischen Zielsetzung festmacht. Beim Begriff „kreative Medien“ ist für Therapeuten und Berater die Tür zur kreativen Verwendung und zur Integration frei geöffnet.

\subsection{Gestaltpsychologische Theorie und der Einsatz kreativer Medien}

\subsubsection{Ganzheitstheorie}

Die Gestalttheorie geht von der primären Ganzheitlichkeit, Strukturiertheit und Dynamik seelischer Gegebenheiten aus, und steht darauf im Gegensatz zu der atomistischen Auffassung (Walter 1994). Gestaltpsychologie stellt das Verfahren der "ganzheitlichen Betrachtung" dar. Ganzheitliche Betrachtung bedeutet, dass man den fraglichen Sachverhalt in seiner Einbettung, in seinem Umfeld, in seiner Rolle und Bedeutung in umfassenderen Zusammenhängen zu sehen versucht; dass man nicht mit eingeengtem Blick immer auf die örtlichen Bedingungen starrt, sondern an die Möglichkeit außerörtlicher Bedingungen denkt (Metzger 1954, S. 13, zit. nach Walter 


\section{Einsatz kreativer Medien in der Gestalttherapie}

1994, S. 20-21). Deshalb ist Phänomenologie ein fundamentales, wertvolles Werkzeug für ganzheitliche Betrachtung, damit man das Umfeld einschließlich seiner Dynamik und seiner Struktur betrachten kann. Das Umfeld beinhaltet die Gesamtsituation, die Bedürfnislage, Einstellung und Haltung des Subjekts sowie seine Geschichte und Erfahrungen. Kurt Goldstein bezog nach seiner Erfahrung im 1. Weltkrieg diese Betrachtungsweise auch auf den Organismus, da jeder Organismus völlig subjektiv das Umfeld erlebt, zu dem er gehört. Seine ganzheitliche Betrachtungsweise ist eine Methode, "die von vornherein das Individuum in den Mittelpunkt der Betrachtung stellte und jedes einzelne Phänomen in seiner Bedeutung für die Existenz des Individuums betrachtete" (Goldstein 1934, X II , zit. nach Walter 1994).

In der Gestalttherapie ist die Ganzheitstheorie besonders mit Bezug auf den Organismus weiter zu verwenden, da ein Organismus als ein aktives Element in dem komplexen ökologischen System des Kosmos zu sehen ist. Deshalb behaupten manche Autoren (z.B. Yontef \& Jacobs 2000), dass die humanistische Theorie der Persönlichkeit ganzheitlich ist. Man kann zwischen dem Organismus und seinem Umfeld unterscheiden, sie aber nicht trennen. Drei Konzepte der Ganzheitstheorie, die Perls in der Gestalttherapie direkt übernommen hat, sind nämlich die organismische Einheit, die spontane Organisation (Selbstregulation) und die Katastrophenreaktion (die Tendenz zum guten Gestalten) (Sherrill 1974). Der Organismus ist „unteilbare Ganzheit“ (L. Perls 1989), physisch und psychisch nicht trennbar. Die Körper-SeeleBeziehung in der Ganzheitlichkeit des Organismus hat die gleiche Bedeutung für die Gestalttheorie. Köhler (1920) sieht es als Isomorphieannahme, „die besagt, dass zwischen physikalischen und physiologischen Vorgängen im menschlichen Hirn und psychischen Prozessen eine strukturelle Übereinstimmung und Wechselwirkung

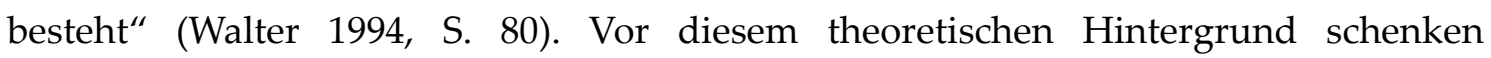
Gestalttherapeuten insbesondere körperlichen Anzeichen eines Klienten ihre Aufmerksamkeit. Nicht nur Emotion, sondern auch Stimme, Haltung, Mimik oder Gestik des Klienten sind wichtige Objekte der Gestalttherapie. Darüber hinaus ist ein Symptom immer in der organismischen Einheit des Klienten zu berücksichtigen, da ein Symptom für den Klienten der bestmögliche Versuch ist, sich den Gegebenheiten anzupassen. In der Wechselwirkung zwischen Organismus und Umfeld entstehen psychische Phänomene, die Goldstein (1934) als zwei Arten von Verhalten kennzeichnet: "geordnetes Verhalten“ und "ungeordnetes oder katastrophales Verhalten“. In der geordneten Situation fühlt sich der Organismus „richtig“ z.B. aktiv, leicht, entspannt und froh, und im Gegensatz dazu in der katastrophalen Situation „unrichtig“, wechselnd, widerspruchsvoll und erschüttert. Diese Unterscheidung 


\section{Einsatz kreativer Medien in der Gestalttherapie}

bedeutet nicht nur im Hinblick auf die Ganzheitlichkeit des Verhaltens und auf die Bezogenheit der einzelnen Vorgänge, sondern auch deswegen, „weil eine Tendenz des Organismus dazu besteht, den katastrophalen Zustand $\mathrm{zu}$ verlassen und den geordneten zu erreichen also eine Tendenz zu geordnetem Verhalten“ (Walter, 1994, S. 23), gewissermaßen „eine Tendenz zur guten Gestalt“ (Metzger 1941). „Tendenz zur guten Gestalt" bedeutet auch Tendenz zur Entwicklung von Sinn und Tendenz zur Ganzheit (Walter 1994). Der Organismus strebt danach, eine möglichst gute Gestalt zu schaffen, um eigenen Sinn im Umfeld zu finden. Somit organisiert sich der Organismus nach seinem Bedürfnis in der Wechselwirkung mit seinem Umfeld als Selbstregulation, da ein Organismus fähig ist, Fehlendes zu kompensieren (vgl. Thomas 1999). Nicht alle Versuche zur Selbstregulation können gelingen. Aber jeder Versuch ist das Beste, was der Organismus im Moment erschaffen kann. Nach der Gestalttheorie ist alles Verhalten durch einen Prozess, nämlich organismische Selbstregulation, die „Homöostase“, reguliert. „Homöostase“ wird als Prozess bezeichnet, in dem der Organismus sein Gleichgewicht zwischen unterschiedlichen Umfeldern gewährleistet. Die Selbstregulation entsteht spontan, richtet sich besonders auf das Ziel der Zufriedenheit des organismischen Bedürfnisses und ist als „ein spontan integrierter und neutraler Teil des Organismus biologisch und psychologisch“ (Korb et al., 1989, S. 11). Das Verhalten des Organismus hat immer in seinem Lebenskontext, seinem Umfeld, was Lewin (1963, S. 87) synonym „Lebensraum“, „psychologischer Raum“ oder „psychologisches Feld“ nennt, mindestens eine Funktion mit dem Ziel zur Selbstregulierung. Gestalttherapeuten interessieren sich für die Funktion, die nicht spontan, sondern immer routiniert ist, weil der Organismus nicht mehr günstig oder lebendig mit seinem Lebensraum in Kontakt kommt und die Möglichkeit zur kreativen Anpassung nicht verwirklicht. Jeder Mensch hat grundsätzlich selbst die besten Fähigkeiten, sein eigenes Leben sinnvoll zu gestalten. Deshalb kann die Aufgabe des Therapeuten nur darin bestehen, ihn bei der Entfaltung der Fähigkeiten zu unterstützen.

Depressive Symptome bei Jugendlichen werden in der Gestaltarbeit folgendermaßen gesehen: Depression ist grundsätzlich ein vorübergehendes Phänomen, das mit oder ohne Hilfe zu einer anderen Gestalt wechseln kann, oder eine Strategie zur Selbstregulierung hat, aber nur wenn das Phänomen für den Organismus nicht mehr als Kompensation gilt. Der Einsatz kreativer Medien hilft im Hinblick auf die Ganzheitstheorie dem Klienten, jeweils alle Anteile seiner Persönlichkeit und ihre Funktionen zur Selbstregulierung wahrzunehmen, zu erfassen und $\mathrm{zu}$ erkennen, sodass er sich als „Einheit Mensch“, nach Goldstein „Organismus“, wieder gewinnen 


\section{Einsatz kreativer Medien in der Gestalttherapie}

und integrieren kann. „Integration“ bedeutet in der Gestatlttherapie aus dieser Perspektive ganzheitliche Harmonie innerhalb des Individuums einschließlich seines Umfeldes. Bei der Arbeit mit Jugendlichen ist der Einsatz kreativer Medien eine gute Möglichkeit, dass sie sich ganzheitlich erleben und am Ende der Arbeit weiterhin Lust zu kreativem Gestalten haben. Dann kann solche psychische Arbeit nicht nur kurativ, sondern agogisch für Kinder und Jugendliche wirken. Kreative Medien erwecken bei Jugendlichen neues Schöpfungspotential, das manchmal in der Schule gestört oder nicht entfaltet werden kann. In der Gestaltarbeit eröffnen sich für Jugendliche durch kreative Medien ganzheitlich verschiedene Kommunikationskanäle, die Lebendigkeit und Kreativität der Jugendlichen beleben.

\subsubsection{Feldtheorie und Zeitperspektive}

Feldtheorie ist wie die Gestalttheorie eine dynamische Theorie. In ihr gibt es keine gegeneinander isolierten, unabhängig voneinander bestehenden Bereiche (Walter 1994, S. 65). Nach Lewin (1963) ist der konkrete Lebensraum, der die Person und ihre Umwelt umschließt, als Feld zu betrachten. „Der Lebensraum, in dem die allgemeinmenschliche Tendenz zur guten Gestalt konkret zum Ausdruck kommt, bietet den Bezugsrahmen, in dem frühere Informationen eingeordnet sind und neue Informationen eingeordnet oder nicht eingeordnet werden" (Walter 1994, S. 83). In dem Lebensraum liegt das Verhalten des Individuums und seine Identität begründet. „Jedes Verhalten oder jede sonstige Veränderung ist einzig und allein vom psychologischen Feld zu dieser Zeit abhängig“ (Lewin 1963, S. 88). Für die Gestalttherapie hat die Aufgabe, Verhalten zu verändern, eine besondere Bedeutung. Die Aufgabe zur Veränderung des Verhaltens ist erstens das Finden einer therapeutischen Repräsentation des Lebensraumes und zweitens die Konkretisierung der Funktion, die das Verhalten mit dem Lebensraum verbindet (Lewin, 1963, nach Walter 1994, S. 85). Im Lebensraum wird die phänomenale Wirklichkeit von Kontext und Kontinuum verbunden. Die Wirklichkeit eines Lebensraumes beinhaltet einen zeitlichen Bestandteil, aus dem Lewin eine Perspektive über "Zeit" von L. K. Frank konzipiert, gewissermaßen die Zeitperspektive (Lewin 1963, S. 116). Lewin definiert: „Die Gesamtheit der Ansichten eines Individuums über seine psychologische Zukunft und seine psychologische Vergangenheit, die zu einer gegebenen Zeit existieren, kann Zeitperspektive genannt werden“ (1963, S, 116-117). Tatsache ist, dass es niemals die Vergangenheit oder die Zukunft als solche sein können, die das Verhalten beeinflussen, sondern immer nur in der Gegenwart existierende Teile des psychologischen Feldes. D. 


\section{Einsatz kreativer Medien in der Gestalttherapie}

h. „Vergangenheit, Gegenwart und Zukunft haben nur in dem Maße und in der Weise Einfluss auf ein Individuum, in dem sie in seinem gegenwärtigen Erleben repräsentiert sind“ (Walter 1994, S. 93). Diese Perspektive hat Gestalttherapie mit dem Motto „Hier und Jetzt" in ihre Praxeologie übernommen und zeigt deutlich den Hinweis auf die Feldtheorie, dass die Korrespondenz der Beziehung Klient-Therapeut im Vordergrund des therapeutischen Feldes steht (vgl. McNamee \& Gergen 1999), da Therapeut sowie Klient nicht nur ein Teil, sondern eine Funktion dessen sind, was momentan geschieht. Die Korrespondenz und Kokreation in der gemeinsamen Wechselwirkung von Klient und Therapeut sind ein wichtiges Fundament der Konstruktion von Gestaltarbeit.

Die Zeitperspektive kennzeichnet vielmehr die tatsächliche Mitte menschlichen Seins. Deshalb kann der Therapeut in der Therapiesitzung vom Klienten wichtige Hinweise aus der Zeitperspektive bekommen. In der Therapiesitzung geht es stets um die Qualität der gesamten Zeitperspektive eines Menschen und aller in die jeweilige therapeutische Beziehung einbezogenen Menschen (also auch der des Therapeuten). Diese Qualität lässt sich letztlich nur daran messen, wie sie zur angemessenen Bewältigung von Vergangenheit, Gegenwart und Zukunft beiträgt (vgl. Walter 1994, S. 99). Deshalb ist die wichtige Aufgabe des Therapeuten in der Gestaltarbeit, wie diese Qualität vom Klienten im therapeutischen Feld dargestellt wird und welche Funktion diese Qualität in seinem Lebensraum hat, da der Organismus und jede Erfahrung, Handlung und Wahrnehmung eine Funktion des gesamten Feldes sind. Diese Qualität des Klienten ist nicht in gleicher Weise bei jedem Klienten messbar, da objektive Wirklichkeit immer eine subjektiv vermittelte ist (vgl. Parlett 1994). Die Qualität kann abschließend messbar sein, da sie sich in jedem Moment verändert je nachdem, wo sich der Klient gerade in Wechselwirkung mit seinem Umfeld befindet. Deshalb überprüft Gestalttherapie mit dem methodischen Schwerpunkt der Praxis der "Awareness" (Gewahrseins) immer die Qualität, wie sie sich gegenüber dem Moment zuvor geändert hat. Ein Hauptthema in der Gestaltarbeit ist die Aufmerksamkeit darauf, was von „Moment zu Moment“ (Yontef 2002) zwischen Therapeut und Klient in dem Feld, in dem sie gerade sind, entsteht. Somit behandelt Feldtheorie das „Wasist", die Organisation der Bedeutung, der Figur und des Hintergrundes und die experimentellen Möglichkeiten von Veränderungen in der Gegenwart.

In der Gestaltarbeit kann der Einsatz kreativer Medien bei depressiven Jugendlichen durch "Gewahrsein" zur angemessenen Bewältigung im Lebenskontext führen. Darüber hinaus werden die Funktionen der Handlung, der Wahrnehmung seines Verhaltens und seines Denkens herauskristallisiert, damit die Art und Weise und Bedeutung der Qualität der Beziehung zwischen Organismus und Umfeld erkannt 


\section{Einsatz kreativer Medien in der Gestalttherapie}

werden können. Lewin (1963) hat auf zwei notwendige Aufgaben zur Veränderung des Verhaltens hingewiesen: a) das Finden einer therapeutischen Repräsentation des Lebensraumes und b) die Konkretisierung der Funktion, die das Verhalten mit dem Lebensraum verbindet. In der Gestaltarbeit mit aktivem Einsatz kreativer Medien bietet der Therapeut den vertrauten Raum für den Jugendlichen an, so dass dieser sein Leben sicher in der Arbeit präsentieren kann. Jugendliche können mit dem Angebot kreativer Medien Lust auf gemeinsame Exploration mit dem Therapeuten bekommen. Sie erforschen mit den verschiedenen Potentialen kreativer Medien die Modalitäten, Funktionen und Bedeutungen ihres Verhaltens, was „die Tendenz zur guten Gestalt" widerspiegelt. Der aktive Einsatz kreativer Medien hilft dabei, diese Phänomene des Verhaltens in ihrem Umfeld und deren Sinn und Funktion mehrfach zu konkretisieren. Während und nach der Konkretisierung des Verhaltens erleben sich die Jugendlichen nicht nur kognitiv, sondern mit ihrem ganzen Leib, in dem sie sich mit seinen Emotionen, Denken und Handeln befinden. Die Konkretisierung des Verhaltens hilft Jugendlichen wahrzunehmen und $\mathrm{zu}$ erkennen, was und wie das eigene Verhalten zur „Tendenz zur guten Gestalt“ beigetragen hat, sodass er weiterhin eine Chance zur guten Gestalt für sich selbst bewahren und somit etwas Neues integrieren und kreieren kann. Der aktive Einsatz kreativer Medien unterstützt nicht nur die Konkretisierung von Problemverhalten, sondern auch die Entdeckung der Ressourcen, der Resilienz, des Potentials und der Stärke des Organismus, der all die Informationen in der Beziehung mit seinem Umfeld im jeweiligen zeitlichen Kontinuum in sich registriert hat.

\subsubsection{Phänomenologie zur Gestaltbildung}

Eine Gestalt zeichnet sich durch Prägnanz aus und hebt sich vom Hintergrund ab. Figur und Grund sind aufeinander bezogen; d.h. sie beeinflussen sich wechselseitig. Wenn eine Figur wahrgenommen wird, wird die Figur nach einer Weile wieder zum Hintergrund. Im Bereich des psychischen Erlebens ist die Figur-Grund-Dialektik vielschichtiger und komplexer. Normalerweise entspricht die Figur in der Gestalttherapie dem menschlichen Bedürfnis. Wenn man sein Bedürfnis vor dem Grund wahrgenommen und es dann erfolgreich erfüllt hat, tritt das Bedürfnis, also die Figur, wieder zurück in den Organismus oder den Lebensraum ein und es zeigt sich eine neue Figur. Eine solche kontinuierliche Eintritt-Rücktritt-Dynamik zwischen Figur und Grund enthält ein Spannungssystem, das zur Erledigung der Aufgabe drängt. Vorher muss ein Organismus zunächst wahrnehmen, ob oder welche Figur sich von 


\section{Einsatz kreativer Medien in der Gestalttherapie}

dem Grund abhebt. Dann überprüft er das wirksame Verhalten, entsprechend dem Bezugssystem, der Handlungsmöglichkeit, der eigenen Fähigkeit zur Erledigung im Zusammenhang mit seinem Lebensraum und passt sein Verhalten wieder in den Grund ein. Unsere Wahrnehmung strebt danach, möglichst prägnante Gestalten zu verwirklichen. Aber eine unverwirklichte oder unvollendete Gestalt (z.B. eine unterbrochene Lebensepisode) hat die Tendenz, sich nicht zu schließen. Diese unerledigten Handlungen werden bevorzugt beibehalten. Das nennt man ZeigarnikEffekt. Zeigarnik (1927) beschreibt ihn als eine „Tendenz zur Wiederaufnahme“ unterbrochener Handlungen.

Beim Figur-Grund-Verhältnis handelt es sich nicht nur um die Tendenz zur Wiederaufnahme, sondern auch darum, ob die Versuchsperson erwartet, die Aufgabe richtig zu lösen. Wenn der Anspruch niedriger und daher nicht mit der Erwartung verknüpft ist, die Aufgabe richtig zu lösen, erlebt die Person die Aufgabe als Ausnahme. Hier spielt zur Erledigung einer Aufgabe, also Figur, der „Ich-Nähe“Faktor eine Rolle. Junker (1960) fand heraus, dass unerledigte Handlungen dann länger im Gedächtnis bleiben, wenn sie ich-näher, also bedeutsamer für die Person sind (Walter 1994, S. 54-55).

Die Prägnanz einer Gestalt ist für Gestalttheoretiker ein wichtiges Thema. Begriffe wie Gestaltqualität (Ehrenfels 1890), Gestalthöhe (Ehrenfels 1916), Prägnanzstufe (Wertheimer 1923) werden gebraucht. Koffka (1935) stellt die „Spurtheorie des Gedächtnisses“ dar: Jedes Erlebnis im Gehirn hinterlässt eine „Spur“. Nach Erklärung von Thomas (1999) trifft auf diese Spurgestalt nun eine neue Wahrnehmungsgestalt, die durch Vergleich wiederum neue Gestaltbildungen in Gang setzt. Dies kann bedeuten, dass sich Spurgestalten verändern im Sinne des "Sich-in-der-MasseVerlierens" oder dass sie prägnanter werden: Die Spurgestalt wird prägnanter als das ursprüngliche Erlebnis (S. 17). Gremmler-Fuhr (2001) stellt die Merkmale von Gestalten im Rahmen der Gestalttherapie zusammen:

a) Transponierbarkeit von Gestalten: Gestalten können in verschiedenen Formen transponiert werden, ohne ihre Qualität zu verlieren.

b) Einbettung in umfassendere Gestalten: Jede wahrgenommene Gestalt erhält ihre spezifische Bedeutung aus ihrem Bezugssystem, ist wieder eine Komponente einer umfassenderen Gestalt.

c) Wechselseitiger Bezug der Komponenten einer Gestalt: Alle einzelnen Komponente der Gestalt sind voneinander abhängig.

d) Dynamik von Gestalten: Gestalten verändern sich ständig.

e) Tendenz zur geschlossenen Gestalt und zur Transformation: Der menschliche 


\section{Einsatz kreativer Medien in der Gestalttherapie}

Organismus hat die Tendenz, sich in eine bestimmte Richtung $z u$ bewegen, eine gute Gestalt zu werden, indem er widersprüchliche und defizitäre Erfahrungen zu lösen versucht (vgl. S. 349-350).

Gestalttherapeuten interessieren sich für das Figur-Hintergrund-Phänomen in Bezug auf die Selbstregulation. Aus diesem Phänomen oder dieser Dynamik entsteht der Kontaktzyklus (siehe. Kapitel 2.1.). Der gesunde Organismus hat die Fähigkeit in dieser Ein- und Rücktrittdynamik, sein Bedürfnis wahrzunehmen, sich damit zu identifizieren, es zu erledigen, Erledigtes loszulassen und Neues zuzulassen, indem er seine Ressourcen in sich oder im Kontakt mit dem Lebensfeld zur Sättigung/Befriedigung effektiv nutzt. Diese Sättigung trägt zur Selbstregulation bei. Wenn man sich in seinem Versuch zur Erledigung nicht satt, sondern gehemmt oder verletzt fühlt, kann eine Spur im Organismus hinterlassen werden. Je mehr diese Spur auf den Organismus einwirkt, umso prägnanter wird das auf diese Spur bezogene Bedürfnis. Kontaktfähigkeit zum Selbst, zum Anderen und zur Umwelt sind unabdingbare Voraussetzungen für einen gesunden Menschen, da Kontakt ein Fundament für menschliches Wachstum ist.

In der Gestaltarbeit steht bei der Kontaktfähigkeit zunächst die Förderung des Kontakts zum Selbst im Vordergrund. Hier wird betrachtet, was in Kontakt zum Selbst kommt, also was vom Selbst als wichtig erachtet wird, z.B. das Bedürfnis nach Nähe, die Sehnsucht nach Wärme oder Anerkennung von der Mutter etc. Hier unterstützen Therapeuten die Fähigkeit zur Identifizierung des eigenen Bedürfnisses, das im betreffenden Moment vom Klienten wahrgenommen wird. In der Gestaltarbeit mit dem aktiven Einsatz von kreativen Medien können Jugendliche zunächst Kontakt zum Selbst nicht nur in verbaler, sondern in verschiedener Weise schaffen und haben die Chance, ihre Gestalten in verschiedene Modi zu transponieren, z.B. in körperlichen, poetischen oder musikalischen Ausdruck. Solche Arbeit mit verschiedenen Ausdrucksformen hilft dem Klienten, nicht abstrakt, sondern konkret mindestens Kontakt zu sich selbst erleben zu können, da die tatsächliche Gestalt durch die Arbeit mit kreativen Medien ausgestaltet wird.

Bei den meisten Klienten, besonders bei Jugendlichen ist die Kontaktfähigkeit zum Selbst, zum anderen, zum Umfeld und zur eigenen Wahrnehmungsfähigkeit gehemmt oder unreif entwickelt. Aber sie haben einen großen inneren Wunsch danach, Kontakt zu ihnen aufzubauen und ihre Fähigkeiten zu verbessern. Aus diesem Grund ist die Gestaltarbeit mit kreativen Medien besonders geeignet, um konkret mit dem eigenen Leib Kontakt während oder nach der Gestaltung aufzubauen und ihre Wahrnehmungsfähigkeit besser $\mathrm{zu}$ entwickeln und die eigenen Grenzen besser 


\section{Einsatz kreativer Medien in der Gestalttherapie}

erkennen zu können. Außerdem haben Jugendliche, die sich gerade mit ihrem stark veränderten Körper unsicher und unwohl fühlen und folglich auch unter einer psychischen Belastung stehen, in der Arbeit die Möglichkeit, sich kennenzulernen und sich mit sich selbst auseinanderzusetzen.

Jedes Gestaltete, also das sichtbare und spürbare Werk eines Klienten, hinterlässt einen konkreten Eindruck auf den Gestalter, also den Klienten. Der Klient als Urheber hat in sich eine Resonanz auf den Eindruck des Gestalteten. In diesem Ablauf zwischen beiden hilft der Therapeut dem Klienten, die Erforschung des Gestalteten für sich in der dialogischen Beziehung unter Verwendung von Wirkpotential und -prinzipien kreativer Medien zu ermöglichen. Wie erwähnt hat eine Gestalt ihre Funktion im Ganzen. Während der Gestaltung mit kreativen Medien hebt sich eine Gestalt, die nicht beliebig ist (Metzger 1962, S. 21), vom Grund ab. Der Urheber einer Gestalt kann sich dadurch ihrer in seinem Lebensraum und im Moment ihres Daseins besinnen. Ihren Sinn und ihre Funktion wahrzunehmen und zu benennen ist ein erster Schritt in der Gestaltarbeit, in dem Phänomenologie und Hermeneutik Zugänge eröffnen. In diesem Arbeitsprozess können Jugendliche den komplexen Zusammenhang zwischen dem, was und wie alles in ihnen, von ihnen und um sie herum aufeinander bezogen und voneinander abhängig ist, besser verstehen, sodass sie zunehmende Selbstverantwortung in sich wachsen lassen können. Auf kreative Anpassung zielt letztlich die Gestaltarbeit bei Jugendlichen ab.

\subsection{Das therapeutische Milieu als Arbeitsgrundlage}

\subsubsection{Förderung der Kreativität}

In der Gestalttherapie ist die Förderung der schöpferischen Anpassung als Therapieziel im Behandlungskonzept verdeutlicht. „Anpassung und Kreativität sind Gegenpole. Sie sind wechselseitig notwendig (Perls et al., 1991, S. 13)“. Zur Anpassung muss man von alter Gewohnheit lassen und neue ungewöhnliche Situationen einlassen. In diesem Prozess benötigt man die Fähigkeiten: Neues zu erkennen und zu entscheiden, ob man im Gewohnten bleibt oder Neues wagen möchte. Während man sein Informationsreservoir aktiviert, sodass die hevorgerufenen Informationen zum Vergleich und zur Einbeziehung in die aktuelle Tätigkeit verfügbar sind, tritt ein schöpferischer Vorgang prozesshaft zutage. Denn es benötigt Kreativität zur Entdeckung von Neuartigem. Schöpferische Menschen verfügen über das entscheidende Maß an Eigengewahrsein, um ihre emotionalen Reaktionen auf 


\section{Einsatz kreativer Medien in der Gestalttherapie}

Information, mit der sie arbeiten, zu erkennen und zu bemerken (vgl. Berley 2006, S. 103), weil Kreativität das Treffen von Entscheidungen und emotionale Resonanzfähigkeit voraussetzt (Damasio 1999). Diese Aspekte weisen darauf hin, dass das Gewahrsein erkannt wird - ob es für sich selbst richtig ist - und die positive emotionale Resonanz die entscheidende Rolle spielen.

Die Fähigkeit, Neues und Ungewöhnliches als solches zu erkennen, ist für den Schaffensvorgang, also zur schöpferischen Anpassung wichtig. „Diese Fähigkeit ist auch dafür von grundlegender Bedeutung, wenn sich Aufmerksamkeit und Gewahrsein des Organismus auf ungewöhnliche Umweltereignisse richten müssen, um Überleben zu sichern“ (Berley 2006, S. 100). Zur Erklärung der schöpferischen Anpassung heben Perls, Hefferline und Goodman (1991) die Struktur von künstlerischer Produktion und Kinderspiel hervor.

„Das Wichtigste an der Psychologie der Kunst liegt in der konzentrierten Wahrnehmung und in der spielerischen Nutzung des materiellen Mediums. Hellwache Wahrnehmung und Spiel mit dem Medium bilden den Kern der künstlerischen Arbeit; so akzeptiert der Künstler seine Träume und benutzt seine kritische Überlegung - und er verwirklicht eine gegenständliche Gestalt spontan. (...) Und genauso ist es bei Kindern: Ihre hellwache Wahrnehmung und ihr freies, scheinbar zielloses Spiel lassen die Energie spontan fließen und so $\mathrm{zu}$ zauberhaften Erfindungen gelangen. In beiden Fällen wirken als Sinnesantrieb die Integration, die Bejahung des Impulses und der wache Kontakt mit neuem Umweltmaterial, aus denen wertvolle Arbeit erwächst. Das sind aber doch ziemliche Sonderfälle. Kunstwerke und Kinderspiele verbrauchen wenig Sozialvermögen und ziehen nicht notwendig zerstörerische Konsequenzen nach sich. Kann dieser Zwischenmodus von Bejahung und Wachsen auch im Erwachsenenleben in ernsteren Bezügen funktionieren? Wir meinen, ja.“ (1991, S. 29-30).

Die Struktur von künstlerischer Produktion und Kinderspiel weist für die Autoren darauf hin, dass hellwache Wahrnehmung, gewissermaßen Gewahrsein oder Awareness und spielerischer Umgang mit dem Medium für schöpferische Anpassung notwendig sind. Aus diesem Grund wird die gestalttherapeutische Praxeologie mit dem Prinzip „Hier und Jetzt“, das Klienten zur Konzentration auf sein Gewahrsein auffordert, konzipiert, damit der Klient seine Kreativität entdecken, aktivieren und entfalten kann. Darüber hinaus entwickelt die Gestalttherapie die therapeutische 


\section{Einsatz kreativer Medien in der Gestalttherapie}

Atmosphäre der Experimentierfreude, damit der Klient ganz ohne irgendwelche Schuldgefühle oder Furcht (vgl. Perls et al. 1991, S. 31) die Elemente ausprobiert und sich zunutze macht. Gestalttherapie unterstützt mit Behandlungskonzepten Lust auf Neues und das Gewahrsein des Klienten, der aus dem nicht mehr funktionierenden Gewohnten heraus nach Neuem sucht.

Die Gestalttherapie hat keine routinemäßige Struktur, die sie durchläuft, sondern sie lässt beständig Neues entstehen. Dieser Prozess bringt schöpferische Veränderung, indem das Leben ständig kreiert und destruktuiert wird. Aus diesem Grund ist Gestalt gewissermaßen wie das Leben. Diese Perspektive gilt auch in der Therapie, wo sich der Klient in der Beziehung mit der Welt findet und Möglichkeiten für seine schöpferische Anpassung und seinen Lebenssinn entdeckt und letztendlich sein Leben neu gestaltet. Das ist das Ziel der Gestalttherapie.

Wie jedes Leben nicht gleich ist, jede Gestalt in „Hier und Jetzt“ einmalig ist, braucht der Gestalttherapeut die Fähigkeit, mit dem Problem des Klienten kreativ umzugehen. Die Lösung eines Problems findet sich weder ausschließlich im Klienten noch ausschließlich in seiner Umwelt, sondern nur in dem Prozess, in dem der Klient mit seiner Umwelt in Kontakt kommt. An der Grenze zwischen Klient und Umwelt gibt es die Möglichkeit, das Problem zu lösen, sich selbst tiefer $\mathrm{zu}$ begegnen und statt Stereotypen Neues zu schaffen. Die Erfahrung fördert die Kreativität. Um kreativ das Leben zu gestalten, ist es nötig, das Erleben und die Erfahrung stark, tief und breit zu machen, da Kreativität davon abhängt, wie die Bereiche des Erlebens erweitert werden können. Kreatives Leben bedeutet, dass das Leben keine Blockierung im Erleben hat, also, dass der Mensch fähig ist, in welcher Situation er nicht stereotypisch mit seinem Verhaltensmuster umgeht, sondern immer neu ausagiert und anpasst (vgl. Kim 1995, S. 111).

In der Gestalttherapie ist der Therapeut, bildlich gesagt der Künstler, mit einem Klienten und der Therapieprozess ein kreativer Prozess. Zinker (1982) meint, dass Therapie und Kreativität miteinander verbunden seien, da sie auf der grundlegenden Ebene zur Transformation, Metamorphose und Änderung gleich seien. Insbesondere ist die Therapie als kreativer Prozess die Transformation einer Form in eine andere, eines Symbols in eine Einsicht, einer Geste in eine Folge von Verhaltensweisen, eines Traumes in eine dramatische Darstellung (vgl. S. 14). Solch therapeutisches Milieu zeigt, dass Entfaltungen der Kreativität für Menschen nicht nur im Sinne der Therapie, sondern überhaupt im menschlichen Wesen fundamental sind. Das steht in der Gestalttherapie im Mittelpunkt. 


\section{Einsatz kreativer Medien in der Gestalttherapie}

\subsubsection{Förderung der Experimentierfreude}

Perls räumt dem unmittelbaren Erleben und Handeln einen größeren Stellenwert ein als dem „Darüber-Reden“. Das Experiment ist ein wichtiges Charakteristikum der Gestalttherapie. Da Perls an vielen künstlerischen Ausdrucksformen interessiert war, wurde der experimentelle und rollenspielerische Zug zur Expression entwickelt (vgl. Rumpler 2007). Die Gestalttherapie fördert die spielerische Atmosphäre und Experimentfreude ihrer Klienten. Zinker(1982) bezeichnet das Experiment als das grundlegende methodologische Werkzeug, ein behavioristischer Ansatz, der auf eine neue Art zu funktionieren zielt (vgl. S. 27). Das Experiment ist die Behandlungstechnik der Gestalttherapie, als „assoziativ-spielerische Selbstexploration der Erlebnis- und

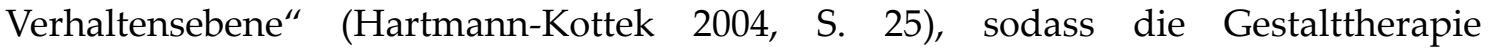
"phänomenologischer Behaviorismus" (Kepner \& Brien 1970) genannt wird , indem Klienten herausgefordert werden, gewöhnliche und vertraute Muster des Verhaltens zu zerstören und eine neue Organisation des Selbst hervorzubringen (Perls et al. 1951). Die übergreifenden Ziele des Experiments sind es, den Bereich des Gewahrseins und des Selbstverständnisses zu vergrößern, die Freiheit, effektiv in seiner Umwelt zu handeln, auszudehnen und sein Verhaltensrepertoire in einer Vielzahl von Lebenssituationen zu erweitern (Zinker 1982, S. 129).

Beim Experiment geht man davon aus, dass man im eigenen unmittelbaren Erleben verankert wird, dass man für sich selbst anschaulich macht, und dass man leicht selbst in die widerstrebenden Seiten, die ursprünglich schwer ins Bewusstsein zu bringen oder anderen mitzuteilen waren, hineingehen kann. Das "Experiment" in der Gestaltherapie ist

„ein Versuch, dem ausweglosen Darüberreden entgegenzuwirken(...). Das Experiment darf nur nicht Linderung bringen oder Ersatz für ein echtes Engagement werden. (....,) Er verbindet die äußere Realität mit seiner inneren Erfahrung, sogar für ihn selbst. Genau das trifft auch auf das therapeutische Experiment zu. Der Betreffende versucht nicht nur, etwas zu reproduzieren, was schon geschehen ist oder was noch geschehen könnte. Stattdessen bezieht er sich auf die äußere Realität, indem er seinen Bedürfnissen zu diesem Zeitpunkt Ausdruck verleiht. Weder probt er ein zukünftiges Ereignis, noch wärmt er ein altes auf, sondern erfährt jetzt in der Gegenwart, wie es ist, den Übergang von der Bewusstheit zur experimentellen Handlung zu schaffen. Wenn er einmal den Rhythmus dieser existentiellen Bewegung spürt, dann 


\section{Einsatz kreativer Medien in der Gestalttherapie}

mag er sich wahrscheinlich anders als vorher in der Welt draußen verhalten“ (Polster \& Polster 1975, S. 220).

Experimente dienen der Verdeutlichung des aktuellen therapeutischen Prozesses oder der Erweiterung und Vermittlung neuer Erfahrungen beim Klienten im „Hier und Jetzt". Im Durchleben neuer Situationen sammelt der Klient neue Erfahrungen an den Grenzen des bisher Gelebten und findet einen neuen Zugang zu seiner Persönlichkeit (vgl. Schneider 1979). Die Wahrnehmung innerer Impulse wird durch das Experiment intensiviert, die der äußeren weitgehend abgeblendet (vgl. Hartmann-Kottek 2004). Während des erforschenden Experiments mit einem Therapeuten wird der Klient mit „existentiellen Ängsten“ (Fuhr 1999) konfrontiert. Es kann sehr bedrohlich sein, mit sich selbst konfrontiert zu werden und die eigenen Tiefen $\mathrm{zu}$ erforschen. Die Wirkung nach dem Experiment ist, dass der Klient etwas Schönes geschaffen hat, etwas, das die eigenen persönlichen Grenzen transzendiert hat (Zinker 1982), sodass der Klient oft nicht nur eine Einsicht durch ein Experiment mit eventueller Konfrontation, sondern auch ein akzeptierendes, wiedererkennendes Schmunzeln erfährt, das man "AhaErlebnis" nennt.

Insofern ist die Gestalttherapie ein experientieller, existentieller und experimenteller Ansatz (vgl. L. Perls, zit. nach Rosenfeld 1978, S.26), durch den therapeutische Arbeit mit Hilfe phänomenologisch-hermeneutischer Erkenntnisweisen durchgeführt wird. Da sich der Erforschungsprozess auf das unmittelbare Erleben bezieht, ist Gestalttherapie experientiell, da immer die Personen in ihrer Lebenssituation und die Auseinandersetzung mit der jeweiligen Umwelt der Inhalt gestalttherapeutischer Arbeit sind. Der Prozeß ist existentiell, da Gestalttherapie die Entwicklung des Lebendigen nicht für determinierbar hält, sondern nur immer wieder etwas Neues versucht werden kann ohne Garantie für den Ausgang dieses Versuches (Fuhr 1999, S. 426). Fuhr betont den Ansatz als Praxisprinzipien der Gestalttherapie mit folgenden Fragen zur Erforschung: Für experientielles Erforschen lautet die Frage „Wie erlebe ich mich?“, für existentielles Erforschen „Wie bin ich in meiner Lebenssituation?“ und für experimentielles Erforschen „Was geschieht, wenn ...?“ (ebd.). Beim Experiment sind solche Erforschungsprozesse verstärkt und miteinander verbunden.

Das Experiment hat keinen beabsichtigten Selbstzweck, der je zur Gänze vorab geplant wird und den der Klient unbedingt erreichen muss. Es bringt in hohem Grad Leben ins JETZT. Von da aus ist es nur mehr ein kleiner Sprung zur Behauptung, dass das Leben selbst ein einziges, ununterbrochenes Experiment sein kann, bei dem die Hier-undJetzt-Welt ständiger schöpferischer Anpassung zum Stoff wird, aus dem das Leben ist 


\section{Einsatz kreativer Medien in der Gestalttherapie}

(vgl. Parlett 2006, S. 63). In dem Experiment gibt es den verbalen Austausch, der die kognitive Grundlage für Änderungen in dem restriktiven Selbstkonzept der Person bildet, verstärkt und ganz in die Person eingeht, so dass es zu einer kreativen Integration der Polaritäten und $\mathrm{zu}$ größerer Vollständigkeit von Erfahrung und Ausdruck (vgl. Zinker 1982, S. 42) kommt.

Perls, Hefferline und Goodman betonen, dass Spontaneität als der innere Kern der Gesundheit gelte. Sie sei das Kennzeichen heilender Einsicht in einer erfolgreichen therapeutischen Sitzung. Wenn man mit Bedürfnissen und Gegebenheiten in Kontakt ist, wird dann im selben Augenblick deutlich, dass die Wirklichkeit nicht starr und unveränderlich, sondern zur Erneuerung bereit ist. Aus diesem Grund betonen sie, dass je spontaner man alle Kräfte der Erkennung und des Zugriffs ausspielt, ohne irgendetwas zurückzuhalten, desto lebensfähiger sich die Erneuerung erweisen wird. Dies fanden die Autoren besonders bei künstlerischer Aktivität und Spiel von Kindern (vgl. Perls et al. 1991, S. 29-30). Nach ihrer Meinung ist Spontaneität für Menschen die Lebensfähigkeit, für sich zu sorgen und eigene Bedürfnisse zu erfüllen. Die Förderung solcher Spontaneität findet man in der Praxis der Gestalttherapie im Experiment. Im deutlichen Kontakt mit eigenen Bedürfnissen und Gegebenheiten muss man Selbstgewahrsein gewinnen und dem vertrauen, was gerade geschieht. Dann wird Spontaneität von deutlichen Kontakt mit den eigenen Bedürfnissen begleitet. Wie, wann und auf welche man sich bezieht, kann man deutlicher auf komplexer Ebene im Experiment erleben.

Da sich das Experiment durch spontanes Handeln und Offenheit auszeichnet, erfordert eine solche experimentierfreudige Haltung ein außerordentliches Maß an Fokussierung bzw. ein hohes Ausmaß an Mut. „Während sich eine Veränderung vollzieht, der kritische Punkt überschritten, ein Höhepunkt erreicht oder überwunden wird, ist die Konzentration hoch. Der Experimentierwille ist ebenso notwendig für die Beständigkeit im Wandel wie für die Herbeiführung des Wandels und für die Förderung des Andersartigen“ (Parlett 2006, S. 66). Deshalb ist es erforderlich, dass der Therapeut den Klienten keinesfalls zum Experiment zwingt, sondern ein Experiment immer in Übereinstimmung mit dem Klienten durchgeführt wird. Im Experiment spielt das Selbstgewahrsein immer die entscheidende Rolle, damit man währenddessen sich selbst und das, was an der Grenze zwischen sich und der Umwelt geschieht, bewusst spüren und sich entscheiden kann, wie man in der Welt sein will. Der Therapeut hilft, an der Grenze zwischen sicheren und gefährlichen Aspekten des Experiments das Gleichgewicht zu halten, indem er die natürliche Entwicklung der unerledigten Themen des Patienten bis zur Vollendung fördert. In diesem Sinne ist die 


\section{Einsatz kreativer Medien in der Gestalttherapie}

experimentierfreudige Haltung, wie schon Amendt-Lyon (2001b) sagte: „No risk, no fun", eine spielfreudige Haltung.

Das gestalttherapeutische Milieu, in dem man mit der Unterstützung und Begleitung des Therapeuten frei experimentieren kann, schafft einen Möglichkeitsraum, der „ein heilsamer Schutzraum" (Hartmann-Kottek 2004), der mit der Atmosphäre des Seindürfens im Sowohl-als-auch ist und dem Klienten hilft, sein derzeit gestörtes Gleichgewicht wieder in seine Mitte schwingen zu lassen. In der experimentellen Situation erfinden Menschen neue Formen des Daseins, wobei Veränderung im Lebensmuster nur dann vorkommt, wenn es genug Stütze aus dem Feld, zu dem der Therapeut gehört, gibt. In diesem Sinne ist ein Experiment die schöpferische Aktivität, die nicht nur bei Patienten angeregt wird, sondern auch beim Therapeuten, damit er sich auch auf sein kreatives Tun und auf seinen Wagemut in der therapeutischen Beziehung einlassen kann (vgl. Rothenberg 1989). Die Zulassung eines Experiments ist also ein Einlassen auf den Lebensfluss, in dem man für eigene Bedürfnisse seine Lebensenergie aktivieren und sein Leben modellieren kann, und ist ein Entwurf (Heidegger), um für sich in der Welt verantwortlich zu sein und in der Welt aktiv mitzuwirken. Selbstverständlich ist auch der Lebensfluss nicht ständig derselbe, sondern immer neu.

\subsection{Chancen und Grenzen der Verwendung kreativer Medien in bestehenden Gestaltarbeiten}

In die Gestalttherapie mündeten die künstlerischen Strömungen von Erfahrungen der Begründer und Begründerinnen der Gestalttherapie ein. Theatererfahrungen bei Fritz Perls, der die bekannte Technik mit dem „Stuhl“ in der Gestalttherapie kreativ weiter entwickelte, Studium in Musik und modernem Tanz bei Laura Perls, die ihr Interesse an Bewegungen und körperlichen Rhythmen später in ihrer therapeutischen Arbeit mit Klienten erklärte und die Veröffentlichung der zahlreichen Literatur und Kritiken bei Paul Goodman, der Gestalttherapie mitten in ein Feld aus ästhetischen Werten und psychologischen Grundsätzen pflanzt. Laura Perls und Paul Goodman, die eine Zeitlang eng zusammenarbeiteten und sich gegenseitig beeinflussten, fanden in der Kunst geradezu das Vorbild für ideale Entfaltung und wandten sie auf alle menschlichen Aktivitäten an (vgl. Miller 2006, S. 174).

Oft vergleichen Gestalttherapeuten Gestalttherapie und Kunst oder künstlerisches Tun, dabei unterstreichen sie besonders die Fähigkeit der Gestalttherapeuten, mit ihrer Kreativität unmittelbar die Therapie zu beeinflussen, weil der therapeutische Prozess 


\section{Einsatz kreativer Medien in der Gestalttherapie}

ohne Kreativität eingeschränkt effektiv ist (vgl. Yontef, 2007, S. 19).

Kreative Medien werden jeweils als „ein geeignetes Hilfsmittel zum Zweck zur Externalisierung und Projizierung der Dynamik der inneren Landschaft und ihres Kräftespiels in die Außenwelt" (Hartmann-Kottek 2004, S. 229) genutzt. Dadurch schaffen sie einen Abstand, der die erneute Hinwendung aus der Distanz erlaubt. Allein dieser Schritt gibt eine erste Entlastung. In diesem Prozess zur Konkretisierung der inneren Kräfte in der Außenwelt zählt Hartmann-Kottek als Nachteil von kreativen Medien eine Reduktion an Nuancen und Vielfalt, aber als großen Vorteil, dass kreative Medien die Hauptdimensionen erkennen und begreifen (vgl. ebd. S. 228).

Es gibt weder eine Methode, die sich als Allheilmittel einsetzen ließe, noch so etwas wie die alleinige Technik zur Behebung einer spezifischen Dysfunktion oder eines Symptoms. Wie Amendt-Lyon meinte, gilt somit auch Gleiches bei der Verwendung der Medien.

„In der Praxis zeigt sich sehr rasch, dass die verschiedenen Matarialien von unterschiedlicher Attraktivität und Wirkung auf verschiedene Patienten sind. Dem Therapeuten obliegt die knifflige Aufgabe, die Kunst und Wissenschaft zu erlernen, verschiedene schöpferische Ausdrucksmittel anzubieten, indem sie ihre Patienten in deren gerade aktuellen, kognitiven und emotionalen Aufenthaltsorten abholt und eine Atmosphäre fördert, in der spielerischer Austausch möglich und ein passendes Mittel für diese Form zwischenmenschlicher Kommunikation gefunden wird“(2006, S. 21).

Hartmann-Kottek (2004) stellt dar, in welchen therapeutischen Phasen kreative Medien von Nutzen sind.

„Sie werden überwiegend im Such- und Präzisionsprozess der Problemkonstellation eingesetzt. Sie helfen aber auch bei einer Bestandsaufnahme, einer Zwischenbilanz. Sie unterstützen das Suchen und Finden einer Lösung und dienen der Verlaufsdokumentation der schrittweisen Veränderung. Bei strukturell schwachen Personen, die also über (noch) kein stabiles Selbstfeld verfügen, hilft die Konfrontation mit dem Zweck des eigenen Ausdrucks dem spiegelnden Selbsterkennen“ (S. 229).

Nach ihrer Meinung werden kreative Medien von Anfang bis Ende mit verschiedenen Einsatzwirkungen in der Gestalttherapie genutzt. D. h. die Verwendung kreativer 


\section{Einsatz kreativer Medien in der Gestalttherapie}

Medien steht nach Bedarf der gestalttherapeutischen Theorie und Praxeologie zur Verfügung.

Kreative Medien sind nicht nur Ausdrucksmittel, die die meisten Therapeuten als Hilfsmittel zum Ausdruck nutzen, sondern auch hilfreich beim Entdeckung verschiedener Potentiale im Prozess entdecken. Sie sind eine Bereicherung des therapeutischen Prozesses und eine Unterstützung in seinem zentralen Anliegen. Die meisten Gestalttherapeuten, die in ihrer Arbeit kreative Medien einsetzen, bemühen sich nicht genug um die Exploration der Identität und der Rolle kreativer Medien in der Arbeit und um die Auseinandersetzung zwischen ihrer Arbeit unter Einsatz von kreativen Medien und der gestalttherapeutischen Theorie und Praxeologie. Wie Perls' Arbeit bei der Entwicklung oder der kreativen Verwendung der Methode mit dem Stuhl aus Morenos Arbeit, ist es notwendig zu erklären, welche Wirkungspotentiale kreative Medien in die Arbeit effektiv einbringen können und wofür und für wen sie in der gestalttherapeutischen Arbeit integriert werden können. Das ist für Gestalttherapeuten ein ziemlich unbearbeiteter Bereich. Einige Gestalttherapeuten (Amendt-Lyon 1999, 2003; Baulig \& Baulig 2002; Blom 2006; Hartmann-Kottek 2003; Oaklander 1978; Sampognaro 2003; Zinker 1982, 2003) versuchen, die Berechtigung der Verwendung kreativer Medien in der gestalttherapeutischen Tradition, die Kreativität unterstützt, zu erklären, aber nicht direkt die Potentiale, Probleme, den Arbeitsprozess und die Besonderheiten der Verwendung kreativer Medien in spezifischen Bereichen der Gestalttherapie. Die Arbeit mit kreativen Medien ist anders als die Arbeit ohne sie. Dabei spielen hier nicht nur die Sprache, sondern auch die verschiedenen Medien, die Klienten und Therapeuten nutzen, in der Arbeit eine wichtige Rolle. In der Arbeit entsteht das gestaltete Produkt von Klienten durch kreative Medien, wonach der Prozess sich anders entwickelt als in der Arbeit ohne kreative Medien. Durch die Vernachlässigung des aktiven Einbezugs der Arbeit kreativer Medien entfaltet die Gestaltarbeit sich nicht, nutzt auch nicht ihre weiteren Entwicklungsressourcen und kreativen Nutzungsmöglichkeiten. Diese Aspekte werden in der vorliegenden Arbeit über den Einsatz kreativer Medien weiter erläutert. 


\subsection{Wirkungspotentiale kreativer Medien in der Gestaltarbeit}

\subsubsection{Zum Ausdruck}

\subsubsection{Katalysator zur Wahrnehmungserweiterung}

Wird unsere Wahrnehmung breit, klar und frei, werden wir offen und fähig für neue Möglichkeiten zur erweiternden und kreativen Anpassung in unserem Lebensfeld. Die Gestaltarbeit mit kreativen Medien fördert die Schärfung und Erweiterung der Wahrnehmung mit dem Stimulus auf der gewohnten Ausdrucksroutine. Durch die Arbeit mit kreativen Medien kann man in die eigenen sinnlichen und leiblichen Wahrnehmungskanäle neue Strömungen einfließen lassen, sodass die erstarrte Wahrnehmungsweise auf verschiedenen Ebenen angeregt wird und man sich schließlich mit seinem Umfeld durch erweiterte Wahrnehmung zur kreativen Anpassung auseinandersetzt.

Erstarrte oder stereotype Muster, festschreibende Narrative und ihre Wiederholungszwänge treten durch kreative Medien im Rahmen gestalttherapeutischer Arbeit in den Veränderungsprozess ein, um generalisierte Fixierungen, erstarrte Muster und Narrative zu verflüssigen (vgl. Orth 1995). Die gestalttherapeutische Arbeit mit kreativen Medien bietet die Impulse in der Weise an, dass der Klient nicht bewusst die „kreative Anpassung“ auswählt, sondern zwangsläufig automatisch, sein kreatives Vermögen mobilisiert, um ihn so zur Erprobung neuer Möglichkeiten und Wege zu ermutigen. Seine Wahrnehmungs- und Handlungsrepertoires während des „einfachen Tuns“ werden mehrfach aktiviert, ohne dass schon eine biographisch aufdeckende, durcharbeitende und verarbeitende therapeutische Strategie zum Einsatz kommt (vgl. ebd. S. 317).

In der Gestaltarbeit mit Einsatz kreativer Medien wird man nicht nur auf der aktiven Wahrnehmungsebene-z.B. auf das Sehen-sondern auch auf der rezeptiven Wahrnehmungsebene-z.B. es fällt ins Auge-angeregt. Die beiden Wege der Wahrnehmung sind schöpferisch und wesentlich und verbunden in einem fließenden Wechselspiel (vgl. Petzold 1999). Aktiv wahrnehmen heißt ergreifen. Z. B. nehme ich wahr, gehe heran, dann ergreife ich die Welt. Rezeptiv wahrnehmen heißt empfangen. Z. B. die Welt kommt auf mich zu, ergreift mich, kommt mir in den Sinn, aber nicht passiv (vgl. ebd.). Es braucht Übung, die Wahrnehmung mit diesen beiden Kanälen, die sich gegenseitig beeinflussen und sich entwickeln, um mit der ganzen Existenz das Sein, das Sein als Verbundenes und das Sein des anderen Menschen zu empfangen und 


\section{Einsatz kreativer Medien in der Gestalttherapie}

zu ergreifen (vgl. ebd.). Ein solcher Wahrnehmungsprozess führt immer zur Resonanz mit sich und der Welt, so dass das Wahrgenommene zum Ausdruck gebracht wird. Da die Gestaltarbeit mit kreativen Medien den Prozess zur Förderung der menschlichen Wahrnehmungs- und Ausdrucksfähigkeit, sozusagen Resonanzfähigkeit, in verschiedener Weise vermittelt, wird man imstande sein, sein Leben neu zu sehen und es schöpferisch zu gestalten.

\subsubsection{Multidimensionales Ausdrucksmittel}

Kreative Medien fördern den Ausdruck von Klienten auf multidimensionale Art und Weise. z.B. olfaktorisch, gustatorisch, kinästhetisch oder auditiv. Was nicht mittels der Sprache zum Ausdruck gebracht werden kann, ist nicht verschwunden, sondern unterschwellig vorhanden, es taucht unbewusst im Leben auf und führt zwangsläufig zur Wiederholung. Solch Verdrängtes, Unterdrücktes, Tabuisiertes, Leidvolles und Bedrohliches, das in unserem Leib auf seinen Ausdruck wartet, braucht eine Schutzinsel, in der es sich sicher und frei von Gefahr ausdrücken kann und folglich losgelassen werden kann. Die Arbeit mit kreativen Medien hilft dem Menschen, die Dinge, die aus verschiedenen Gründen nicht zum Ausdruck gebracht werden können und noch im Leib geblieben sind, sicher zum Ausdruck zu bringen.

Hier steht die menschliche Fähigkeit zur kreativen Projektion, die sich von pathologischer Projektion unterscheidet, dem Klienten zur Verfügung. Nach Zinker (1982) werden Projektionen in drei Schritten zu kreativen Projektionen. Zunächst lernt der Mensch zu erkennen, was er in der Welt fürchtet. Dann beginnt er einen Dialog zwischen den Polaritäten in sich, findet zu einem freundlichen Wohlwollen gegenüber all den polarisierten Kräften in sich und fängt an, seine Ganzheit zu erleben. Schließlich transformiert der Mensch ein Dilemma in ein konkretes Produkt oder eine konkrete Handlung. Der kreativ Projizierende macht die Energie seines Konflikts nutzbar. In diesem Sinne ermöglicht es die Arbeit mit kreativen Medien dem Menschen, in Kontakt mit der Energie zu kommen, die im Konflikt „eingefroren“ ist, und sie für die Verwirklichung des Selbst und der Menschheit verfügbar zu machen (vgl. Zinker 1982). Das bedeutet, dass die Arbeit mit kreativen Medien dem Klienten dabei unterstützt, dass es seine in sich eingefrorene und blockierte Energie auf verschiedene Weise zum Ausdruck und ins Fließen kommt, was ihm ursprünglich nicht im Bewusstsein war.

"Gestalten als Ganzheiten des Erlebens zeichnen sich dadurch aus, dass sie in andere Ausdrucksformen transponiert werden können, ohne ihre wesentliche Qualität zu verändern“ (Meier 1990, zit. nach Fuhr 1999). Das Transponieren ist eine fruchtbare 


\section{Einsatz kreativer Medien in der Gestalttherapie}

Möglichkeit für den Wechsel und die Eröffnung einer neuen Perspektive in sich selbst. So können bewusste Erlebnisse in andere Formen des Ausdrucks umgewandelt werden. Deshalb kann es sinnvoll sein, die Erlebnisse in für Klienten ungewohnte Formen des Ausdrucks verwandeln zu lassen, sie zu malen, plastisch zu gestalten usw. (vgl. Fuhr 1999), sodass man durch Verfremdung gegenüber den gewohnten Ausdrucksformen seine Welt multidimensional neu entdecken kann. Da ein Prozess nicht nur allein wichtig ist, sondern der Einfluss verschiedener Prozesse aufeinander bedeutsam ist, wie Petzold (1974) es mit den Begriffen Synopse und Synergie zum Ausdruck bringt, erreichen wir das therapeutische Ziel durch die Wechselbeziehung zwischen kreativen Prozessen und kreativen Medien, die in multidimensionalem Vorgehen genutzt werden.

\subsubsection{3 Ästhetisches Erfahrungsmittel}

Das Tun mit kreativen Medien ist ein schöpferischer Prozess, um sich davon zu überzeugen, in der Welt zu sein, und die Welt auf sich einwirken zu lassen. Das bedeutet, dass man durch das kreative Tun sowohl sich selbst als auch die Umwelt gestaltet.

„Kann ein Mensch sich wirklich selbst wahrnehmen, erleben, erfahren, so kann er eigentlich nicht umhin, sich selbst zu gestalten. Hier liegt der Wirkfaktor künstlerischer Therapie und Therapie mit kreativen Medien“ (Petzold 1999, S. 21). Während des kreativen Tuns kommt man in Kontakt mit der eigenen Welt, nimmt sie wahr und gestaltet ein Symbol seiner inneren Welt, die sich immer erneut verändert. Nach der kreativen Gestaltung erforscht man sich von, über oder mit dem gestalteten Produkt aus kreativen Medien. Das Erforschen zum Selbst führt in einem solchen therapeutischen Prozess immer zu einer neuen Gestaltung, was als „die vitale Daseinserfahrung" (Panofsky 1932, zit. nach Petzold 1999q) und „Lebenskunst“ (Foucault 1993; Deleuze 1993) bezeichnet wird. Ein solcher Versuch der Selbsterforschung und -gestaltung ermöglicht die Entfaltung der eigenen schöpferischen Existenz. Hier braucht man den „mehrperspektivischen Blick“ (Petzold 2003) für sich selbst, um seine Vielfalt zu erkennen und zu verwirklichen, da wir uns nach der Erfahrung von Ganzheit, nach vollendeter Form und Integration sehnen (vgl. Zinker 2003). Das führt zu der Erfahrung des Eingewobenseins des Selbst in Kontext und Kontinuum. Es gibt uns die Chance, in die Lebensgeschichte und ihre Reflexion mit ästhetischer Erfahrung und ästhetischem Verhalten hineinzugehen und das eigene Selbst in Beziehung zum Kontext zu erkennen (vgl. Petzold 1999q, S. 21). 


\section{Einsatz kreativer Medien in der Gestalttherapie}

Miller unterstreicht die ästhetische Transformation psychischen Leidens (1980) in der Therapie und Ästhetik der Hingabe (2003), die er von den beiden Künstlern Paul Cézanne und Miles Davis als „Wichtiges“ für ein besseres Leben übernommen hat. Die Suche nach dem bestmöglichen Ausdruck der einzigartigen Subjektivität (ebd.) ist für Menschen wesentlich, erforscht Hingabe des Menschen an die Aufmerksamkeit und Kreativität, „das Gute zu wollen“, und führt zu Transzendenz und Transformation, also Metamorphose, indem man seine Grenzen akzeptiert und versucht, sie weiter auszudehnen.

Die Gestaltarbeit mit kreativen Medien gibt dem Klienten Anlass und Chance, sich im Sinne der „vitalen Daseinserfahrung“ und der „Lebenskunst“ wahrzunehmen, sich zu erforschen und sich schließlich als "gute Gestalt" zu schaffen, so dass der Klient die ästhetische Erfahrung auf sich einprägen lässt und seinen Erforschungsprozess im ganzen Leben hindurch ästhetisch und kreativ weiterführt. Ästhetische Erfahrung und Schaffensvorgang in der Gestaltarbeit mit kreativen Medien werden an den Grenzen zwischen Individuum und Produkt, oder im zwischenmenschlichen Raum, in dem der Klient mit dem Therapeuten arbeitet, geboren. Die Gestaltarbeit mit kreativen Medien treibt sinnhaft den Erforschungsprozess und den Selbstgestaltungsprozess voran. In diesem Sinne ist Gestaltarbeit mit kreativen Medien sowohl kreativer Prozess (vgl. Zinker 1982) als auch ästhetischer Prozess.

\subsubsection{Zur Erfahrungsanreicherung mit dem gestealteten Produkt}

\subsubsection{Der Zugang zum „eigenen archaischen Land“ des Klienten}

Da die meisten Menschen in unserer Kultur Teile des Sensorisch-Physchischen und Emotionalen verdrängt oder abgespalten haben, müssen die Phänomene der Verdrängung oder Abspaltung erst wieder dem bewussten Erleben zugänglich gemacht werden. Hier spielen „kreative Medien“ eine wichtige Rolle, da sie mit ihrem eigenen Charakter zur Gestaltung, die sichtbar, spürbar und eventuell tastbar sind, dem Klienten helfen, sich seine eigene Welt zugänglich zu machen. Die eigene Welt, die für den Klienten sprachlos, unerreicht und unzugänglich war, wird mit und durch kreative Medien ein Geschöpf, das nur darauf wartet, mit dem Klienten in Kontakt zu kommen, um benannt und bearbeitet zu werden, also eine Resonanz zu bekommen.

In der Gestalttherapie geht es nicht darum, dem lange Zeit Unterdrückten zum Durchbruch zu verhelfen, sondern das Unterdrückte und Verdrängte soll dem Bewusstsein in einer Weise zugänglich werden, dass es der Klient in sein Selbst- und 


\section{Einsatz kreativer Medien in der Gestalttherapie}

Weltbild integrieren kann (vgl. Fuhr 1999). Damit das Unterdrückte, Verdrängte und Abgespaltene dem Bewusstsein zugänglich werden kann, muss es sich zunächst zeigen und sich differenzieren, wie es ist. Der Klient sollte damit in Kontakt kommen. Diesen Kontakt zum eigenen Land des Klienten ermöglicht in sinnvoller Weise die Arbeit mit „kreativer Medien“.

„Kunsttherapeutische Methoden und Medien haben in dialogischer Absicht das Ziel, Zugang zu derartigen archaischen Skripts und Narrativen $\mathrm{zu}$ finden, indem sie das projektive Potential medialer Arbeit und die in Gestaltungsprozessen zum Tragen kommenden Symbolisierungen nutzen" (Petzold \& Orth 1993a, S. 153). Das mit kreativen Medien hergestellte Produkt entsteht durch Symboliserung der präverbalen Welt, oder der Welt, die nicht sprachlich zum Ausdruck gebracht werden kann. Solche Symbole evozieren bei Klienten meistens Erstaunen und Faszination über das Gestaltete, welches gerade vor ihnen entstanden ist. Dies wiederum führt zur Lust, das Produkt und seine Aussage über das Selbst weiter zu erforschen, zu erfahren, was das Symbol bedeutet und wie es sich auf sie selbst bezieht. Darüber hinaus hat der Klient nicht nur die Neugierde auf sich selbst in Bezug auf das Produkt, sondern auch auf das Erforschen, d.h. der Klient erforscht sich selbst als ein Objekt-distanziert, aber nicht abgespalten. Diese Distanz kann am Anfang notwendig sein, um die Sinnzusammenhänge des Erlebens zu entdecken, ohne von den Empfindungen und Gefühlen, die das Erleben begleiten, überwältigt oder zu stark involviert zu werden. Dabei wird nicht die kognitive Distanziertheit angestrebt, sondern eine emotional und empfindungsmäßig eingebundene Distanz (vgl. Fuhr 1999, S. 429). Das Ausmaß der Distanzierung, Identifizierung und Involvierung auf das Produkt ist abhängig von dem Prozess, der Bereitschaft des Klienten und dem Kontext. So fängt der Klient eine Reise in seine innere Welt mit sicheren Hinweisen aus dem Gestalteten an.

\subsubsection{Das Mittel der dialogischen Selbstreflexion}

Die Arbeit mit Medien produziert Gegenstände in verschiedenen Situationen, in der Menschen ihren bestimmten Lebenskontext, Erinnerungen, Gefühle und Handlungsweisen hervorrufen. Das Gestaltete evoziert etwas aus den „Leibgedächtnissen und Leibarchiven“ (Petzold 1996), in denen all die Erfahrungen und Materialien bewusst und unbewusst gespeichert sind. Während und nach der Gestaltung spiegelt das Produkt die hervorgerufenen Erfahrungen wider, spricht den Klienten an, wer und wie sie sind und was sie für den Klienten bedeuten. Durch die Hinweise des Gestalteten ist der Klient auf dem Weg zur Besinnung seines Lebens. Das 


\section{Einsatz kreativer Medien in der Gestalttherapie}

Gestaltete ist eine ergreifbare Aussage, führt zu Aktionen, die einen erweiterten Dialog sowohl mit sich selbst als auch mit anderen schaffen (vgl. Polster \& Polster 1975, S. 222), und zur persönlichen Hermeneutik des Subjekts (vgl. Petzold \& Orth 1985, S. 59).

Dieser erweiterte Dialog mit sich selbst ist ein wichtiger Weg zur Selbstreflexion. Das Produkt vermag es dem Gestalter zu ermöglichen, sich mit der Botschaft des Gestalteten und seiner Beziehung dazu auseinanderzusetzen. Diese Fähigkeit von Medien nannte Petzold (1988n) die der "Autokommunikation“. Medien dienen der Übermittlung von oder sind Systeme von Informationen im Rahmen der Methode des therapeutischen Arbeitens. „Ihre Kommunikationsfähigkeiten werden im Rahmen der therapeutischen Arbeit mit kreativen Medien oder kunsttherapeutischer Arbeit nicht nur auf der Seite intragruppaler und zwischenmenschlicher Kommunikation, sondern auch der Autokommunikation“ (Petzold 1988n) gefördert. Wenn ein Klient z.B. eine Figur mit dem Medium Ton hergestellt hat, wird „eine Botschaft der Figur von sich, über sich, für sich und an andere“ (Petzold 1988n, S. 52), wird der Dialog mit sich selbst deutlich und bewirkt, dass unbewusstes Material zutage kommt (Petzold \& Metzmacher 1984; Petzold \& Orth 1985; Petzold 1988n).

So ein Dialog mit sich selbst ist die Voraussetzung der menschlichen Fähigkeit zur Exzentrizität. Der Eindruck des Produkts lädt uns in eine bestimmte Szene oder Situation ein, in der wir gefühlsmäßig und atmosphärisch sensibilisiert werden. Durch Identifizierung mit dem gestalteten Objekt oder einem Teil des Objektes und durch die exzentrische Exploration konstelliert sich das Gefühl für eine persönliche Kohärenz. Kann das Individuum ein Muster und eine Struktur des Phänomens des Gestalteten erkennen, selbst wenn das Phänomen zwar anscheinend chaotisch aussieht, ermöglicht der Identitätsprozess durch seine Arbeit, dass Kontinuität der Lebensgeschichte, Kohärenz des Persönlichkeitserlebens und Sinnerfahrung für das Individuum ermöglicht werden (vgl. Osten 2000). Die selbstreflexive Beschäftigung ist ein wichtiger Bestandteil der Personwerdung und Identitätsbildung, aber Selbstaufmerksamkeit allein zeigt eher schädigende Wirkungen. Aus diesem Grund begleitet der Therapeut den Prozess des Klienten.

\subsubsection{Die Boje zum neuen Anfang}

Ein gestaltetes Produkt, welches der Klient in der Gestaltungsphase mit kreativen Medien hergestellt hat, und welches als Symbol und Selbstsystem seiner inneren Welt willkürlich gestaltet wurde, ist der Gegenstand, der den Klienten zur Resonanz und Auseinandersetzung auffordert. Des Weiteren birgt es die Möglichkeit für den Klienten, 


\section{Einsatz kreativer Medien in der Gestalttherapie}

$\mathrm{zu}$ kommunizieren und weiter verarbeiten $\mathrm{zu}$ können, und hat die Funktion der Begleitung des Prozesses, in dem der Klient die Erfahrung mit dem Produkt in sich tragen und verarbeiten kann. Dann ist das im Gestalten und im Produkt Erfahrene eine konkrete Evidenz, mit der der Klient im Nachhinein seinen Prozess zur Veränderung nicht nur in seiner Erinnerung, sondern wahrnehmbar, spürbar erleben kann. Das bedeutet, wie ein Schmetterling seine Puppe sieht, kann der Gestalter erfahren, wie und woraus er in den Fluss der Veränderung kommen kann. Den gleichen Sinn hat die Hausaufgabe, ein Therapietagebuch zu führen.

Dazu ein Beispiel: Nachdem ein Klient mit einer Puppe, welche als ein Symbol seines in der Familie vernachlässigten inneren Kindes steht, gearbeitet hat, nimmt er sie mit nach Hause. Dort nimmt er durch die Puppe hervorgerufene Erinnerungen und Bedürfnisse aus seiner Kindheit wahr und kümmert sich nun darum. Dabei steht die Puppe für das Kleinkind. Sie ist eine Evidenz dessen, was er in der Therapie herausgefunden hat, und ein konkreter Ausweis des Lebens oder des Therapieprozesses. Dabei befriedigt er im Nachhinein nicht nur die eigenen Bedürfnisse, sondern vermag sein Potential und seine Ressourcen frei zur Verfügung zu stellen. Ein weiteres Beispiel: Während der Klient mit seinem Therapeuten mit einem unter Schmerzen selbst gemalten Bild arbeitet, sieht er nicht nur die Schmerzen, sondern auch seine Lebenskraft, mit der er diese schmerzhafte Situation durchstehen kann. Nach der Sitzung kann er aufgrund seiner Erfahrung mit dem Bild seine Kraft spüren, sodass er das Bild, anders als früher, nachmalen kann. Das Bild, das zuerst gemalt wurde, ist eine Boje zum neuen Anfang in dem Veränderungsprozess, welcher immer weiter fließt.

$\mathrm{Da}$ solche Gestaltungen wegen ihrer sinnlichen und konkreten Ausdrucksmöglichkeiten vielseitig ansprechend sind, kann der Klient durch das Gestaltete im Nachhinein mit seiner Identifikationsfähigkeit, aber auch mit seiner Abstandsfähigkeit etwas Neues, ein neues System oder eine neue Verbindung zu sich herausfinden. Diese kreativen Techniken bieten Klienten durch die Vergegenständlichung psychischer Inhalte in der Regel einen höheren Grad an Exzentrizität gegenüber sich selbst und ihren Themen (Petzold \& Osten 1999). Die Exzentrizität fördert, dass durch Wechselwirkung der Prozesse eine spiralartige Entwicklung entsteht, alles mit allem zusammenhängt und sich gegenseitig beeinflusst. Wenn solche Erfahrungen angereichert werden, kann der Klient mit der Begleitung des ganzen Produkts in seinem Veränderungs- oder Wachstumsprozess die wiederkehrenden Elemente und Erlebensweisen (vgl. Osten 2000), also Narrative, deutlich erkennen. D. h. er kann einen Umriss seines Lebens und seiner 


\section{Einsatz kreativer Medien in der Gestalttherapie}

Lebensbiographie erkennen, seinen Lebenssinn gewinnen und ein Gefühl von Kohärenz, Kontinuität und Identität entwickeln, indem er mit sich, mit seiner Biographie und mit seinem Umfeld sicher im Kontakt bleibt. Dann beginnt er eine neue Geschichte mit neuer Zukunftsperspektive für sein Leben zu schreiben.

\subsubsection{Zur Diagnostik und der Informationserhebung}

Es ist bekannt, dass circa 80 Prozent der Diagnostik von schwierigen Kindern nicht haltbar sind (vgl. Pohl 2002). Solches trifft auch mehr oder weniger bei Jugendlichen zu. Diagnostik ohne Therapie oder Beratung verteilt "Stempel“ und vermittelt kein Wohlbefinden (vgl. Baulig \& Baulig 2002). Diagnostik setzt immer Therapie oder Beratung voraus, sie ist nicht einmalig erhebbar, da sich der Zustand des Klienten immer verändert. Was könnte sich anbieten, um zu einer effektiven Diagnostik bei Kindern und Jugendlichen zu gelangen? Als eine mögliche Antwort auf diese Fragestellung nennen Diagnostiker den Einsatz von projektiven Medien.

Die Arbeit mit kreativen Medien führt zu einem projektiven Prozess. Projektionen sind eine kreative Fähigkeit des menschlichen Wesens. Sie sind keineswegs nur als psychopathologische Phänomene oder als Abwehrmechanismen zu sehen, sondern „als gesunde Möglichkeiten der Aufgabenbewältigung, des Gestaltungspotentials, der Informationsverarbeitung von Menschen, als Artikulation emotionaler und mentaler Verarbeitungsprozesse, Ausdruck persönlicher Kreativität bzw. Kokreativität“ (Petzold 1971k, zit. nach Müller \& Petzold 1998). Solche Projektionsfähigkeiten des Menschen nutzen Kliniker insbesondere für die Diagnostik bei Kindern, deren innere Welt mittels

Sprache nur schwer zur Verfügung steht, und entwickelen verschiedene projektive Testverfahren zum Zweck der Diagnostik und der Informationserhebung bei Kindern, um unbewusste, motivationale und emotionale Dimensionen erschließende projektive Aspekte in Diagnostik und Therapie einzubringen.

Während des projektiven Arbeitsprozesses mit kreativen Medien tauchen zahlreiche Informationen auf, die die verschiedenen Facetten der Persönlichkeit des Klienten enthalten bei denen nicht nur Probleme und Defizite zu sehen sind, sondern auch Potentiale, Ressourcen und Resilienzen. Deshalb wurden verschiedene projektive und semiprojektive Verfahren, die sich von der standardisierten Vorgabe projektiver Tests unterscheiden, mit dem Einsatz von kreativen Medien zur Diagnostik in der Integrativen Therapie entwickelt, wie "Lebenspanoramen“, "Netzwerkdiagramme", „Identitätssäulen“ oder „Body Charts“ (Petzold \& Orth 1991a; 1993a; 1994). Durch sie ist es möglich, dass Klient und Therapeut die mit der Themenstellung verbundenen 


\section{Einsatz kreativer Medien in der Gestalttherapie}

Informationen, sowohl vom Produkt oder Ergebnis als auch vom Gestaltungsprozess, diagnostisch und therapeutisch vielfach neue Wahrnehmungs-, Verarbeitungs- und Handlungsmöglichkeiten erschließen und konstellieren können. Aus diesem Grund sind „Diagnostik und Therapeutik unlösbar miteinander verwoben. Das gilt für die mediale Arbeit noch stärker als bei verbalen Explorationen, denn die Medien aktivieren das Erleben und bieten unbewusste Dynamik und Ausdrucksmöglichkeiten. Medien werden als Träger oder Systeme von Informationen in einem kommunikativen Prozess verstanden“ (Orth 1995, S. 328).

Die Informationen werden nicht nur von den gestalteten Objekten, sondern auch von dem Gestaltungsprozess oder dem gesamten Therapieprozess erfasst. Die Diagnose wird nicht nur zu Beginn der Therapie einmalig gestellt, sondern in jedem Moment, in dem sich der Klient befindet. „Each moment is seen as a hologram for the larger whole of the patient's life. This perspective gives guidance to diagnostic questions, and in turn is guided by diagnostic understanding or understanding of the particular character logical pattern of the patient" (Yontef 2002). Deshalb findet die Diagnose prozessual und kontinuierlich bis zum Ende der Therapie statt. Die vom Produkt und Prozess erhobenen Informationen tragen zur Diagnostik bei, die Diagnostik ist deshalb nicht vom Therapieprozess getrennt, denn der Klient und seine Umwelt verändern sich ständig während der Therapie und während seines gesamten Lebens. Insofern sind „Diagnose und Therapie ... derselbe Prozess“ (Perls et al. 1979, S. 243) und der Klient ist der Kodiagnostiker (vgl. Osten 2000) und Phänomenlieferant (vgl. Baer, 2004), da die Diagnose in der Gestaltarbeit nicht nur vom Therapeuten allein in der Therapie erschlossen wird, sondern in der interaktiven Beziehung mit dem Klienten immer für neue Veränderungsmöglichkeiten geöffnet wird.

Kreative Medien sind für die Untersuchung der psychischen Situation und der Persönlichkeitsstruktur von Klienten, insbesonders bei Kindern und Jugendlichen, besonders gut geeignet. Sie sind nicht bloß Träger einfacher Botschaften, sondern „ein sehr komplexer Informationsbündel, die zu Symbolen verdichtet gesendet werden" (Richter 2003, S. 63), weshalb in der Arbeit mit kreativen Medien nicht nur ihr Inhalt, sondern auch ihre Struktur und ihr Prozess, also phänomenologisch und hermeneutisch in der korrespondierenden Beziehung zwischen Klienten und Therapeuten verarbeitet werden muss. 


\section{Einsatz kreativer Medien in der Gestalttherapie}

\subsubsection{Zur Eröffnung des Zwischenraums}

Ein therapeutischer Raum, der dem Klienten zum Durchführen der Experimente und zur Selbst- und intersubjektiven Explorationen ausreichend, sicher und schöpferisch zur Verfügung steht, ist in der Arbeit mit kreativen Medien basal. Empfindet der Klient diesen Raum als unangemessen, resultiert daraus eine Verarmung des Wachstumspotentials und eine Einschränkung der Aktivität der Innenwelt des Klienten. Der „potentielle Raum“ (Winnicott) ist der „Übergangsraum“ (Wheeler, 2006), von dem die Qualität kreativer Prozesse beim Experimentieren und Erproben abhängt. Es ist der "wachstumsfördernde Raum", in dem der Klient seine eingeschlossene Lebensenergie entdecken und wieder frei fließen lassen und seine Grenze erweitern kann, der „Festraum", in dem er sich durch seinen Erfahrungsprozess und die in dem Prozess entstandenen Produkte mit Vertrauen und Verlässlichkeit geschmeichelt fühlt, und der „Lernraum“, wo der Klient für sich, über sich, über das, was eben geschieht, und mit einem Blick wohlwollender Neugier auf das eigene Leben gewinnbringend lernen kann.

Es ist die Besonderheit dieses Ortes, an dem Spiel sich ereignet, dass er existentiell von der lebendigen Erfahrung abhängt und nicht von Anlagefaktoren (vgl. Winnicott 1971). Nach Winnicott umfasst der Ort Geborgenheit, Zuwendung und Objektangebot, so dass die Menschen maximale persönliche Reifung bei der Integration, der psychosomatischen Zusammenhänge und der Beziehung haben können. Medien haben den Charakter von Übergangsobjekten (Winnicott 1973) oder von Intermediärobjekten (Rojas-Bermúdez 1983). Kinder nutzen in der Trennung von ihrer Mutter das Übergangsobjekt, das sie statt der Mutter(brust) tröstet, bis sie sich selbst von der Subjekt-Objekt-Welt unabhängig entwickeln.

Hier existiert ein potentieller Raum, wo Kinder das Leben kreativ und spielend erleben, indem sie mit dem Übergangsobjekt spielen, ohne Unterdrückung und Einschränkung der Selbstverwirklichung. „Dieser intermediäre Erfahrungsbereich entwickelt sich direkt aus dem Spielbereich, bleibt lebenslang für außergewöhnliche Erfahrungen im Bereich der Kunst, der Imagination und der schöpferischen wissenschaftlichen Arbeit erhalten“ (Winnicott, 1973, S. 23-25).

„Herrscht in einer Beziehung Vertrauen und Verlässlichkeit, so entsteht ein potentieller Raum, ein Raum, der zu einem unbegrenzten Bereich der Trennung werden kann, den das Kleinkind, das Kind, der Jugendliche und Erwachsene kreativ mit Spiel erfüllen kann, aus dem sich später Freude am 


\section{Einsatz kreativer Medien in der Gestalttherapie}

kulturellen Erbe entwickelt. Es ist die Besonderheit dieses Ortes, an dem Spiel ... sich ereignet, dass er existentiell von der lebendigen Erfahrung abhängt und nicht von Anlagefaktoren“ (Winnicott 1971, S.126).

Dieser schöpferische Raum und der Heilraum können sich auf die "schöpferische Indifferenz" (Friedlaender 1918) beziehen. Während des Gestaltens und der Bearbeitung bleibt der Klient wachsam im Zentrum, dort kann er

„eine schöpferische Fähigkeit erwerben, beide Seiten eines Vorkommnisses zu sehen und jede unvollständige Hälfte zu ergänzen. Indem er eine einseitige Anschauung vermeidet, gewinnt er eine tiefere Einsicht in der Struktur und die Funktion des Organismus“ (Perls 1978, S. 19).

Moreno (1946) bezeichnet den Verlust der Kreativität als Kreativitätsneurose. Die Kreativität als eine wichtige Ressourcen- oder Resilienzquelle (Krüger 2004) muss geschützt und gefördert werden. Die Gestaltarbeit mit kreativen Medien schafft die spielerische, freie Atmosphäre, die zur Entwicklung oder Entdeckung der eigenen Kreativität beiträgt.

In diesem Sinne ist der Raum, der in der Gestaltarbeit mit kreativen Medien entstanden ist, ein "Proberaum", in dem der Klient seine Handlungen und Möglichkeiten zum Coping oder neues Verhalten erprobieren kann, ein „Schöpfungsraum", in dem er sich selbst als gute Gestalt erkennt, versteht und den eigenen Lebenssinn entdeckt, damit man sich selbst neu gestalten kann, und ein „Erlebnisraum“, in dem er mit kreativen Medien den verborgenen Teil seines Selbst neu erfahren kann und defizitäre oder mangelnde Erfahrungen oder geschädigte und schmerzhafte Erfahrungen in der Beziehung mit einem Therapeuten als korrigierbar erlebt. Aus diesem Grund ist er ein „Zwischenraum“, in dem der Klient erproben oder sich üben kann und wo die Realität und die Phantasie, Bewusstes und Unbewusstes sich begegnen 


\section{Teil II: Entwicklung, Anwendung und Evaluation des Programms zur indikativen Prävention von Depression}

\section{Konzeptentwicklung für die Gestaltarbeit mit kreativen Medien}

\section{bei depressiven Jugendlichen}

\subsection{Arbeitskonzepte}

\subsubsection{Entwicklungspsychologische Perspektive}

Die Behandlung psychischer Störungen in der Adoleszenz ist noch weitgehend ein weißer Fleck auf der gestalttherapeutischen Landkarte. Auffällig ist, dass Fragen der Behandlungstechnik bei Jugendlichen nicht selten zusammen mit Fragen der Behandlungsmethode bei Kindern abgehandelt wurden, als gäbe es keine entwicklungspsychologischen Unterschiede (vgl. Seiffge-Krenke 1986). Eine solche Situation herrscht nicht nur bei der gestalttherapeutischen Schule, sondern bei allen Therapieschulen vor. Ich gehe davon aus, dass beispielweise ein Klient mit Depressionen in verschiedenen Lebensaltern nicht in gleicher Weise behandelt werden kann. Aus diesem Grund werden Fragen der Behandlungskonzepte einschließlich der Behandlungsmethode und -technik hier einen besonderen Schwerpunkt ausmachen. Kindliche und jugendliche Störungen sind nach Schuch (2000) dadurch gekennzeichnet, a) dass sich ihre Verhaltensauffälligkeiten häufig situativ bedingen und nicht Ausdruck stabiler Persönlichkeitseigenschaften sind, b) dass ihr Verhalten innerhalb eines breiten Spektrums zu unterschiedlichen Ausprägungsgraden einzelner Verhaltensmuster führt, und c) dass manche ihrer Symptome als Schwierigkeiten in der Bewältigung von altersgemäßen Anforderungen und nicht als Krankheit zu bewerten sind. Dies ist dann der Fall, wenn ihre Verhaltensauffälligkeiten nicht durch im Rahmen der Psychopathologie definierten Kriterien zu erfassen sind, und Beeinträchtigungen in relevanten Lebensbereichen mit sich bringen bzw. weitere Entwicklung gefährden (vgl. S. 406-407).

Der Mensch ist in der Reifezeit des Jugendalters ein einsames Wesen durch das Bewusstwerden seiner Vereinzelung durch Geburt und Konstitution, durch das 


\section{Arbeitskonzept für die Gestaltarbeit mit kreativen Medien}

Schicksal der Individualhistorie und des sozialen Milieus (vgl. Nissen 1971). Da Jugendliche gerade in verschiedenen Lebensräumen nach der Entfaltung ihrer Interessen und Kompetenzen streben, sollte die Jugendarbeit die Situation der Jugendlichen unter Berücksichtigung ihres Umfeldes beurteilen. D.h. sie muss in ihre Überlegungen mit einbeziehen wie die Jugendlichen sich zurechtfinden: in der Beziehung mit ihrer Familie, ihren Freunden und in der Lebenswelt, der sie angehören (z.B. in der Schule).

Die Gestaltarbeit mit kreativen Medien gibt den Jugendlichen die Möglichkeit, die Wechselwirkungen von sich und anderen in ihrer Lebenswelt $\mathrm{zu}$ begreifen, $\mathrm{zu}$ verstehen und schliesslich $\mathrm{zu}$ integrieren. Dabei können sie Konflikte, Probleme, Vulnerabilität und Bedürfnisse bemerken und sich in ihren Lebensräumen weiterentwickeln. Darüber hinaus gibt die Gestalttherapie ihnen die Chance, die Qualität der verschiedenen Lebensräume zu bewerten und zu erforschen, in welchem Lebensraum und mit wem sie die schädlichste bzw. fröhlichste Zeit hatten oder haben, was der schlimmste oder beste Lebensraum und Mensch $\mathrm{zu}$ ihrer Entwicklung beigetragen hat oder nicht.

Die Jugendlichen befinden sich in einer körperlichen und dementsprechend geistigen Wandlungsphase, in der die Entwicklung ihrer körperlichen, psychischen und sozialen Identität gefordert wird. Heutige Jugendliche haben dazu noch Schwierigkeiten, z.B. Auseinandersetzungen mit familiären Situationen, in der sie ggf. verschiedene Rollen als Familiemitglieder haben müssen (z.B. „Ich bin ein Sohn von meiner Mutter, von meinem ersten Stiefvater, bin ein Stiefbruder von der Tochter meines zweiten Stiefvater" etc.), und in der sie ggf. weniger als sonst von den Eltern Aufmerksamkeit bekommen wegen gesellschaftlicher Anforderungen an Erwachsene, z.B. mehr für das Geld arbeiten zu müssen. Aus diesem Grund können unsere Jugendlichen mehrfach von der Gesellschaft in ihrer Entwicklungsaufgabe geschädigt werden, so dass ihre Verhaltensauffälligkeiten im Vordergrund stehen.

Aber bei Verhaltensauffälligkeiten muss der Therapeut oder Berater darauf achten, dass die Arbeit nicht nur auf und an Symptomen oder Problemen der Jugendlichen fokussiert und orientiert ist, sondern auch ihre Ressourcen, Potentiale und Resilienz in den Blick nimmt, da ihre Probleme Anzeichen eines komplexen menschlichen Wesens, das enorme Entwicklungsmöglichkeiten hat, darstellen. Deshalb dürfen die Probleme der Jugendlichen nicht einseitig gesehen werden, sodass die Arbeit zu weiteren Entwicklungsmöglichkeiten der Jugendlichen führen kann.

In diesem Sinne zielt die Gestaltarbeit mit kreativen Medien ganz darauf ab, ihre Ressourcen und protektive Faktoren $\mathrm{zu}$ explorieren, $\mathrm{zu}$ aktivieren und $\mathrm{zu}$ entfalten. 


\section{Arbeitskonzept für die Gestaltarbeit mit kreativen Medien}

Darüber hinaus erfüllt die Gestaltarbeit mit kreativen Medien die hohe Selbsteffizienzerwartung, die als ein altersspezifisches Thema der Jugendlichen in der Therapie angesehen wird. Sie suchen beim ersten Gespräch Möglichkeiten, ob die Therapie ihnen etwas zu geben vermag, und sie überprüfen, ob die Therapie ihre Kompetenzerwartung ignoriert, respektiert oder sogar tangiert. Deshalb ist das erste Gespräch ein wichtiger Faktor, um ihre Motivation und Bereitschaft zu erwecken, und es sollte so durchgeführt werden, dass Rücksicht auf ihre Empfindlichkeit und ihre Angst vor Verletzung genommen wird. Jugendliche sind im Erkennen und im verbalen Ausdruck psychischer Phänomene, in der Einsicht in den Zusammenhang ihres Verhaltens und der Bedeutung vom Leben eingeschränkt, und die Ausdrucksfähigkeiten der Jugendlichen bei bestimmten Symptomen, z.B. Angst oder Depressionen, sind besonders gehemmt. Unter Berücksichtigung der entwicklungspsychologischen Perspektive wird die Gestaltarbeit mit aktivem Einsatz kreativer Medien zur indikativen Prävention depressiver Jugendlicher konzipiert und durchgeführt.

\subsubsection{Gruppenarbeitssetting}

In der Gestalttherapie wie bei anderen Schulen ist noch nicht eindeutig erforscht, für wen und wann eine gruppentherapeutische Maßnahme nach bestimmter Diagnostik bei Kindern und Jugendlichen durchgeführt werden sollte. Beim Gruppensetting scheint der Vorteil zunächst zu sein, dass es ökonomischer und damit kostengünstiger ist als das Einzelsetting. Baulig und Baulig (2004) meinen, dass eine GestaltGruppentherapie mit Kindern nach einer Einzeltherapie geboten sein kann, wenn z.B. Kontaktprobleme mit Gleichaltrigen bestehen oder soziale Anpassungsprobleme in der Einzeltherapie nicht hinreichend bearbeitet bzw. thematisiert werden können. Hier kann das Setting zur Veränderung der thematischen Fokussierung beim Kind beitragen. Aus ihrer Sicht gibt es zwei Varianten gestalttherapeutischer Gruppenarbeit mit Kindern: Die zu therapeutischen Zwecken organisierte Gruppe und die gestalttherapeutisch bestimmte Arbeit mit bestehenden Gruppen (z.B. der Schulklasse). Bei der ersten Gruppe werden die Temperamente und Polaritäten von Kindern berüchsichtigt, damit vermieden wird, dass bestimmte emotionale Tendenzen dominieren. Bei der zweiten Gruppe wird ein hohes Konfliktlösungspotential durch gestalttherapeutisch orientierte Interaktionen gefördert. Diese Gruppenarbeitsform ist für Jugendberatung und Schulhilfe als grundsätzlich positiv einzuschätzen (vgl. S. 33). In der vorliegenden Gestaltarbeit unter aktivem Einsatz kreativer Medien ist die 


\section{Arbeitskonzept für die Gestaltarbeit mit kreativen Medien}

Jugendberatung zur Bewältigung oder indikativen Prävention von Depressionen konzipiert. Aus folgenden Gründen ist das Arbeitssetting als Gruppe mit dem Ziel indikativer Prävention oder der Bewältigung von Depression gut geeignet: die Betroffenen sind nicht schwer geschädigt, sie brauchen die Bestätigung von anderen insbesondere von Gleichaltrigen - dass sie nicht unnormal sind, und sie befinden sich in einem Entwicklungsprozess, wo sich ihre Fähigkeit hinsichtlich der Themen „Nähe und Distanz", "Abhängigkeit und Unabhängigkeit", „Erkennen der eigenen Grenzen“, „Kontakt zu sich selbst, zu anderen und zum Umfeld“ verbessern können. In der Gestaltarbeit mit kreativen Medien erleben die Jugendlichen nach der Gestaltung mit den Medien Resonanz und Feedback von anderen, was für das menschliche Wesen immer wichtig ist, sodass eine neue Perspektive entsteht, in der sie sich widerspiegeln können. Beim gegenseitigem Feedback oder beim Resonanzgeben fühlen sie sich kompetent, anderen helfen zu können, so dass sie sich durch Geben und Bekommen als wertvoll erleben können. Ein solcher Prozess bezieht sich ganz klar auf die Entwicklung ihrer Identität. Identität entsteht immer in der Wechselwirkung zwischen der Art, wie ich von anderen gesehen werde und wie ich mich selbst sehe. Ist eine Kluft dazwischen, bemüht sich der Mensch, die Kluft zu beseitigen. Wenn dieser Versuch nicht gelingt, befindet man sich in einem Konflikt. Deshalb sagt Petzold (1982), dass die Therapie der „Identitätsarbeit“ dienen sollte. Gruppensetting ist zum Zweck der Identitätsentwicklung besonders bei Jugendlichen zu bevorzugen.

Im Unterschied zu Franck (1997), der die Konfliktaustragung und das Sich-Finden in den Vordergrund der therapeutischen Arbeit stellt, sind Baulig und Baulig (2004) der Meinung, dass Gestalt-Gruppentherapie mit Kindern einer Rahmenvorgabe bedarf (vgl. S. 33-34). Sie schlagen folgende therapeutische Struktur vor: 1. Eingangsritual 2. Problembearbeitung aus dem Hier-und-Jetzt 3. Von den Therapeuten vorgegebene Themen 4. Abschlussrituale.

Die vorliegende Gestaltarbeit mit dem aktiven Einsatz kreativer Medien ist nach vorgegebenen Themen für 10 therapeutische Sitzungen, zuzüglich einer zur Einführung und einer für die Rückmeldung in Bezug auf eine spezifische Behandlung von Depression konzipiert. Der gesamte Prozess ist dreigeteilt 1. erlebnisfördernde Exploration 2. aktiver Ausdruck mit dem Gestalteten 3. Integration. Jede Einzelsitzung kann je nach Gruppenprozess anders gestaltet werden, aber grundsätzlich sollen fünf Phasen ablaufen: 1. Aufbau 2. Gestaltung 3. Intensität 4. Zusammenführung oder Eröffnung neuer Wachstumsdimensionen (siehe. 4.2 und 4.3 in dieser Arbeit). 


\section{Arbeitskonzept für die Gestaltarbeit mit kreativen Medien}

\subsubsection{Erlebnisfördernde und ausdrucksorientierte Arbeitsmodalitäten}

Die in der vorliegende Studie zur Arbeit mit depressiven Jugendlichen eingesetzten Kommunikationskanäle sind außer der Sprache: verschiedene Medien, die in unterschiedlicher Weise wirken können, sowie die „intermediäre Arbeit", die von Petzold und Orth am Fritz Perls Institut konzipiert und entwickelt wurde. Neben der körperliche Sprache steht das Malen, neben dem Malen steht die Poesie, danach z.B. das Puppenspiel. In einer solchen Arbeitsweise wechseln die Kommunikationskanäle, mit denen sich Jugendliche nicht prototypisch zum Ausdruck bringen und evozieren auf verschiedenen Weisen ihre Sensibilität und Kreativität. In der vorliegenden Arbeit werden Malen, Tonerde, Poesie, Schnur, die von Teilnehmern selbst mitgebrachten Materialien, das symbolisierte non-verbale Spiel, Musik zur Einstimmung etc. eingesetzt. Die von den Teilnehmern in einer solchen Arbeit entstandenen Werke sind für sie ein „Intermediärobjekt“ (Rojas-Bermúdez 1983), was in der Arbeit mit Puppen hervorgehoben wird, welches uns an das „Übergangsobjekt“ Winnicotts (1971) erinnert. Das „Intermediärobjekt" ist weder in der Realität noch in der Phantasie vorhanden, vermittelt aber den Teilnehmern ihre innere Welt, um Kontakt zum Selbst, zu anderen oder zum Umfeld aufzubauen und einen Dialog zu führen.

In der Therapie oder der Beratung Jugendlicher steht verbale Kommunikation in Form von spontanem und unzensiertem Ausdruck kaum zur Verfügung. Da die Jugendlichen oft nur ein begrenztes kognitives Niveau der Einsichtgewinnung haben, stellt der therapeutische Prozess mit seiner Betonung von verbalem Austausch und Reflexion eine potentielle Bedrohung dar, führt in der therapeutischen bzw. beraterischen Arbeit mit Jugendlichen dazu, dass die Kommunikation mit dem Therapeuten bzw. dem Berater häufig durch Schweigen und Unvermögen zur Verbalisierung von Gefühlen und Gedanken gekennzeichnet ist (Steinhausen, 1993), und folglich zu einem Versagen der Therapie bzw. Beratung, also Drop-Out. Wenn das therapeutische Konzept nicht darauf achtet, wie Jugendlichen das Kommunizieren erleichtert wird, bleiben unsere Jugendlichen, die dringlich Hilfe brauchen, immer in einem Teufelskreis, in dem sie außer dem psychischen Symptom noch zusätzlich belastet werden. In einer amerikanischen Studie (Armbruster \& Kazdin 1994) wird nachgewiesen, dass immerhin etwa 50\% der Kinder und Jugendlichen die Behandlung abbrechen. Es ist nicht schwer abzuschätzen, dass es solche Tendenz nicht nur in den USA gibt. Wenn Kliniker, Therapeuten und Berater trotzdem solche Tatsachen vernachlässigen, arbeiten sie mit Jugendlichen aber nicht für Jugendliche. Jugendliche befinden sich heutzutage nicht nur in einer Situation, in dem sie sich mit ihrer Identität 


\section{Arbeitskonzept für die Gestaltarbeit mit kreativen Medien}

zwischen Kinder- und Erwachsenenwelt beschäftigen, sondern auch in einer Krise, in der sie nur die Option haben, eine Therapie bzw. Beratung entweder für Kinder oder für Erwachsene auswählen zu können, wenn sie wegen irgendeines Problems fachliche Hilfe brauchen.

Das vorliegende Konzept mit kreativen Medien ermöglicht den Aufbau einer Brücke, über die eine Verbindung zwischen Jugendlichen und Therapeuten bzw. Beratern und verallgemeinert zwischen dem Erwachsenland und Jugendland hergestellt werden kann. Die Jugendlichen müssen dort abgeholt werden, wo sie sind, und es soll nicht dazu kommen, dass die Jugendlichen dem Therapeuten dahin folgen, wo er ist. In der Arbeit mit der Verwendung von kreativen Medien können die Zugänge zum Erleben, zur Beeinträchtigung des Selbstwertgefühls, zu Anteilen der Persönlichkeit und zu Konflikten auf intra- und interpersonaler Ebene gefunden werden, die sich Jugendlichen oft bei einer verbalen Verarbeitung nicht erschließen. Bei dem Ausdruck dessen, was unterdrückt geblieben ist, bei der Suche nach der richtigen Ausdruckform, fühlen sich die Jugendlichen erleichtert, frei und kompetent. Diese Erfahrung bringt ihnen eine neue Konstellation des Gefühls und eine Einsicht, die später synergetisch in ihrem Leben weiter wirken kann.

\subsubsection{Einsatz des gestalteten Produkts statt des Stuhls}

Die hier entwickelte Gestaltarbeit mit kreativen Medien zur indikativen Prävention von depressiven Jugendlichen ist nach Grundprinzipien der Gestalttherapie und vom spezifischen Behandlungskonzept nach der EFT konzipiert. In der EFT werden verschiedene "Stuhltechniken“, gemäß dener die Aufgaben bestimmt werden, für die Behandlung von „depressiven Erwachsenen“ - nicht Jugendlichen - verwandt, und wurden als erfolgreich nachgewiesen. In dieser Gestaltarbeit wird überlegt, ob und wie dieses Behandlungskonzept mit "Stuhltechniken“ auch bei Jugendlichen erfolgreich angewandt werden kann. Zur Konzipierung der Arbeit zur indikativen Prävention von depressiven Jugendlichen ist $\mathrm{zu}$ berücksichtigen, dass die Arbeit fokal und gruppenförmig angewandt, dass sie nach Merkmalen des Jugendalters auf die Entwicklungsperspektive hin konzipiert wird, und dass sie unter Rücksichtnahme auf die Eigenschaft der depressiven Jugendlichen in der Beratung und Therapie geschieht. In dieser Arbeit wird das dargestellte Produkt, das die Teilnehmer in der Arbeit gestaltet haben, statt der Stuhltechnik zum aktiven Ausdruck verwandt. In der Gestalttherapie und der EFT kommen die "Stuhltechniken“ in mindestens drei Hauptthemen zur Anwendung: Selbstbewertungsspaltung (z.B. Selbstkritik), 


\section{Arbeitskonzept für die Gestaltarbeit mit kreativen Medien}

selbstunterbrechende Spaltung (z.B. Gefühlsblockierung) und unterbrochene Prozesse oder Gefühle gegenüber bestimmten anderen Menschen (siehe Kap. 2.2.1.4.). Der Stuhl wird in der Anwendung im Rahmen der Gestalttherapie ein Wesen, ein Platz oder ein bestimmter Mensch in der Phantasie des Klienten evoziert. Auf dem Stuhl inszeniert der Betroffene seine innere Landschaft bezüglich der Spaltung zum Selbst oder „bringt“ eine bestimmte Person des unterbrochenen Prozesses „auf den Stuhl“. Bei der Selbstbewertungsspaltung, in der mit zwei Stühlen gearbeitet wird, unterstützt der Therapeut den Betroffenen zunächst in der Arbeit bei der Trennung von beiden polaren Selbstaspekten und zur Identifizierung der polaren Seiten, sowie bei der selbstunterbrechenden Spaltung zur Trennung von beiden relevanten Selbstaspekten und zur Differenzierung der selbstunterbrechenden Aspekte. Dafür ist die Fähigkeit des Therapeuten sehr wichtig, vorausgesetzt wird aber auch die Fähigkeit auf der Seite des Betroffenen zur Trennung der polaren Seiten, der Identifizierung und der Inszenierung.

Für depressive Jugendliche bedeutet ein solches Arbeitskonzept mit der "Stuhltechnik" eine hohe Belastung und viel Vorstellungsfähigkeit. Ich gehe hier davon aus, dass die Arbeit mit kreativen Medien für eine kurzzeitige Gruppenarbeit durch die Gestaltung mit kreativen Medien und mit dem gestalteten Produkt durch kreative Medien, die in der Gestaltung entstanden sind, für die Behandlung von Depressionen von Jugendlichen erfolgversprechend ist. Durch die Gestaltung mit kreativen Medien können Jugendliche in mehrfacher Weise ihr Erlebnis vertiefen. Das Erlebnis trägt später zur erhöhten Motivation und zum aktiven Engagement bei.

Die Verwendung des gestalteten Produkts hat das Potential (siehe Kap. 3.5.), die Inszenierung innerer Selbstspaltungen und Darstellung des „unterbrochenen Gefühls" zu einer bestimmten Person ohne Mühe ausdrücken zu können, da das Produkt hilft, konkret und leicht in Kontakt zu kommen. Beim aktiven Ausdruck drücken die Teilnehmer ihre innere Spaltung und ihre unerledigten Dinge mit Hilfe des Produkts aus, das sie in den Sitzungen mit Blick auf ihre Lebensräume - z.B. Schule und Familie - gestaltet haben. Das Gestaltete kann das Gesicht einer Person, die für den Klienten einen unterbrochenen Prozess oder ein unterbrochenes Gefühl hervorgebracht hat, sein. Es kann aber auch ein Gegenstand, auf den sich die Selbstspaltung bezieht, oder ein Zeichen des Gefühls sein. Statt dass sie die Dinge auf dem Stuhl inszenieren und vorstellen, erleben sie mit ihrem Gestalteten eine weitere Intensität. Ein weiterer Grund, für die Bevorzugung der Gestaltarbeit mit dem Gestalteten statt der "Stuhltechnik“ ist, dass das Gestaltete keine Angst hervorruft. D.h. das Gestaltete gewährleistet einen sicheren Abstand oder die Exzentrizität in sicherer 


\section{Arbeitskonzept für die Gestaltarbeit mit kreativen Medien}

Weise. Die Teilnehmer müssen dabei kein Schamgefühl haben, sich zu zeigen. Im erlebnisfördernden Explorationsprozess werden die Teilnehmer bereit, um in der Arbeit tief in sich hineinzugehen, wobei nicht nur die Probleme aktualisiert werden, sondern auch das Reservoir der Ressourcen. Nach dem Einlassen auf diese Ressourcen arbeiten die Teilnehmer mit Abstand konkret an dem Produkt. Die gestalteten Gegenstände können getastet, gespürt oder gesehen werden. Das bringt für die Teilnehmer konkreten Kontakt zum Gestalteten, worin die eigenen Gefühle, bestimmte Personen oder sie selbst symbolisiert werden. D.h. die Teilnehmer können ihre Kontaktfähigkeit $\mathrm{zu}$ sich selbst, $\mathrm{zu}$ anderen und zum Umweltfeld verbessern oder wieder beleben, damit eine kreative Anpassung realisierbar wird.

\subsubsection{Haltung des Therapeuten oder Beraters}

Grundsätzlich ist der Therapeut oder Berater in der Gestalttherapie oder -beratung nach der Feldtheorie "ein Teil der Therapie oder Beratung“ (Parlett, 1994, S. 127). Das ist eine umfassendere Sicht des therapeutisch Relevanten. Die Theorie sieht vor, dass der Therapeut oder Berater als Mitschöpfer der Systeme arbeitet: „Indem wir die feldtheoretische Perspektive akzeptieren, erkennen wir, dass wir nicht nur IN einer Gruppe sind, sondern dass wir die Gruppe SIND, nämlich ein holographischer Teil von ihr. Wir tragen die Systeme, in denen wir leben, und sie sind Teil unserer Existenz, genauso wie wir ein Teil von ihrer Existenz sind“ (ebd. S.129). Deshalb ist die Beziehung zwischen Therapeut bzw. Berater und Klient das Medium der Heilung. Der Gestalttherapeut muss vollkommen anwesend, authentisch, offen gegenüber Überraschungen, anpassungsbereit gegenüber den Umständen, und fähig sein als „Willenbrecher", als der „Organisator" einer beständig sich verändernden, gemeinsam geteilten Realität zu fungieren. Und er muss von nicht nachlassender Neugierde sein gegenüber den zum Vorschein kommenden Bedeutungen, eingebauten Annahmen und verankerten Projektionen, die sich in den offenbar werdenden Erfahrungen der Teilnehmer erkennen lassen, und in höchstem Maße respektvoll, sowohl gegenüber der je eigenen Komplexität des Hintergrundes, der Lebensumstände und charakterlichen Strukturen jeder einzelnen Person als auch gegenüber deren gegenwärtigen Versuchen, jetzt das Beste aus ihren Erfahrungen zu machen (vgl. Parlett 1994, S. 230-232).

Im Rahmen der Gestaltarbeit mit Jugendlichen sollte der Therapeut oder Berater besondere Fähigkeiten haben. Zunächst braucht er Kenntnisse über die Probleme, die mit bestimmten Alters- und Entwicklungsstufen verbunden sind, und Interesse für die Jugendkultur und -sprache, was heutige Jugendliche modisch bevorzugen, und 


\title{
4 Arbeitskonzept für die Gestaltarbeit mit kreativen Medien
}

Rücksichtnahme gegenüber dem Umfeld von Jugendlichen und seiner Funktion, z.B. wie die familiären Systeme von Jugendlichen funktionieren und sie beeinflussen, welchen Einflüssen der Schule Jugendliche ausgesetzt sind, usw. Für effektive Kommunikation muss der Therapeut besonders dahingehend vorsichtig sein, direkt mit dem Jugendlichen umzugehen, ohne aufdringlich $\mathrm{zu}$ sein und sich klar, unmittelbar und unkompliziert auszudrücken (vgl. Oaklander, 1978).

Der Therapeut, der mit kreativen Medien arbeitet, muss keine perfekte Kompetenz bei deren Verwendung haben, aber die Eigenschaften der Medien gut kennen (vgl. Oaklander, 1978). Amendt-Lyon (1999) meint, dass die Gestalttherapeuten mit einer Vielfalt von Medien vertraut sein und kompetenten Beistand bei der Auswahl kreativer Kanäle und wachstumsfördernder Interventionen leisten sollten (vgl. S. 862). Die wohlwollende Neugierde als Haltung des Therapeuten oder Beraters ist nicht genug. Eine unbefangene Haltung, Demut und Akzeptanz gegenüber dem Dasein sind wichtige Haltungen des Therapeuten oder Beraters. Der Therapeuten oder Berater sollte die Veränderung der Klienten einschließlich der therapeutischen Beziehung beobachten, miterleben und sich darüber mitfreuen und an der Veränderung des Klienten, die in jedem Moment stattfindet, glauben. Yontef (2007) betont Folgendes: "The therapist facilitated this by identifying the thoughts and feelings as they emerge moment-to-moment, by formulating the theme emerging from these moments, and respecting the patient's core emotions and his defenses" (S. 20). Miriam Polster (2003) erklärt die Leitlinien für die Therapie mit zwei Metaphern in einem Interview:

\begin{abstract}
„Manchmal habe ich das Gefühl, dass ich durch meine therapeutische Arbeit einen Abszess aufschneide, so dass kranke Materie abgestoßen und die Wunde gereinigt wird, damit das Gewebe wieder heilen kann. Das seelische Gewebe des Patienten kann anfangen zu heilen. [.... Es ist eher so, als ob ich versuche, ein Feuer wieder ans Brennen zu bringen. Hier und da sehe ich ein paar Funken und sage mir, dass da etwas Lebendiges ist, das man wieder anfachen kann. Ich blase Luft hinein, um es wiederzubeleben. Häufig geht es darum, das Leben, das Lebendige zu suchen und zu unterstützen“ (S. 207).
\end{abstract}

Die Haltung soll gewährleisten, dass der Gestalttherapeut einerseits Verletzungen, Probleme oder Defizite des Klienten, und andererseits seine Ressourcen im Auge zugleich hat.

Folgende Haltung des Therapeuten oder Beraters ist in der Gestaltarbeit mit dem Einsatz kreativer Medien bei Jugendlichen unabdingbar: dass der Therapeut oder 


\section{Arbeitskonzept für die Gestaltarbeit mit kreativen Medien}

Berater zunächst die Souveränität des Klienten respektiert, und dass er sein Wesen und sein Gestaltetes mit kreativen Medien nicht schnell interpretieren darf. In dem Prozess begleitet der Therapeut den Klienten immer, ist nicht vor oder hinter ihm. Jugendliche Klienten haben oft Angst vor dem Versuch von Therapeuten, sie und das Gestaltete vorschnell und übergreifend zu verstehen. Ein solches Verhältnis verletzt den Klienten und beraubt ihn seiner Motivation und seines aktiven Engagements bei der Arbeit, die sowieso ohne Reflexion von ihm weiterläuft. Deshalb ist die gemeinsame Erforschung auf beiden Seiten notwendig. Hier weist Yontef (2007) in Bezug auf die Haltung des Therapeuten oder Beraters hin, wenn der Therapeut oder Berater die Absicht zur Veränderung der Teilnehmer hat, bekommt der Klient die Botschaft, dass der Therapeut oder Berater ihn besser als der Klient selbst kennt. Dann entsteht eine abhängige Beziehung, wobei der Klient seine Souveränität verliert und keine gesunde Kommunikation stattfinden kann. Auf eine solche Haltung muss der Therapeut oder Berater, der besonders mit Kindern und Jugendlichen arbeitet, verzichten. Hat der Therapeut oder Berater die Haltung des Zulassens des menschlichen Wesens, das imstande ist, sich im Moment zu verändern, und glaubt er, dass jeder Mensch die Fähigkeit hat sich zu verändern, eine gute Gestalt zu schaffen, bekommt der Klient durch diese Haltung die Gelegenheit, die eigenen Kräfte zur Veränderung oder Problembewältigung zu entdecken und sich als ein wertvolles Wesen neu zu sehen, wenn er ganzheitlich er selbst bleibt und mit sich in Kontakt kommt.

Der Therapeut oder Berater muss ganz der Resilienz des Klienten vertrauen. Die Resilienz entfaltet sich natürlich, wenn man mit dem Hier und Jetzt vollkommen in Kontakt bleibt. Der Therapeut oder Berater muss intersubjektiv mit dem Klienten auf ein Verstehen seines menschlichen Wesens hin arbeiten. Er fühlt mit dem Klienten mit (Mitfühler), erforscht gemeinsam mit ihm (Miterforscher), sucht mit ihm nach seiner Schatzkiste (Mitentdecker), ermöglicht die Bereitstellung seiner Ressourcen (Beleber) und fördert ihn, sein Leben neu zu konstruieren. Er unterstützt den Prozess seines Klienten, indem er Gedanken und Gefühle, die der Klient von Moment zu Moment hat, identifiziert, Themen aus diesem Moment formuliert und fundamentale Gefühle des Klienten und seinen Widerstand respektiert (vgl. Yontef 2007). In diesem Prozess ist er ein Cokreator (White 1993), eine Hebamme (Simkin 1976), da sich durch ihn als Katalysator neue Gestaltungsmöglichkeiten für den Teilnehmer eröffnen. 


\section{Arbeitskonzept für die Gestaltarbeit mit kreativen Medien}

\subsection{Behandlungsprinzipien}

\subsubsection{Grundprinzipien}

\subsubsection{Förderung des Gewahrseins des „Hier und Jetzt $t^{\prime}$}

"Gewahrsein (Awareness)“ ist ein Zustand und eine Fähigkeit, die durch Kontakt mit der Existenz entsteht. Er ist dynamisch und zyklisch, indem ein Mensch Kontakt zur sensorischen Wahrnehmung oder zum Selbstbedürfnis herstellt und dadurch anderen oder vertieften Kontakt möglich macht. Dieses Verhältnis ermöglicht dem Menschen Verantwortung dafür, wer er ist und was er tut. Perls meint mit Gewahrsein die Fähigkeit, mit der eigenen Existenz in Berührung zu kommen, zu erklären, was im Moment in einem und um einen passiert, sich mit der Umwelt zu verbinden, und zu wissen was man fühlt, empfindet und denkt, also wie man im Moment reagiert (vgl. Clarkson \& Mackewn 1994). Gewahrsein kann durch Variation der Erfahrungen und des Experiments während des Prozesses der Gestalttherapie erlebt werden. Indem die Arbeit mit kreativen Medien dem Klienten verschiedene Bereiche des Gewahrseins und verschiedene Sinne anregt oder erregt, kann der emotionale Ausdruck eine „essentielle Form“ (Clynes 1976, zit. nach Dreitzel 1995) finden. Gewahrsein umfasst nach Frühmann (1994, S. 144) drei Ebenen: eine interne, die man Selbstbewusstheit bzw. Selbstwahrnehmung nennt, eine externe, die die Wahrnehmung des anderen und der Welt ist, und eine mediative, die Perls ",awareness of what's between- the intermediate zone“ (1969, S. 49) genannt hat, die der Bereich der kontaktverhindernden Projektionen und Abwehrmechanismen ist. Hier trägt die Arbeit mit kreativen Medien als Stimulus zur feinen Wahrnehmung und zu ihrer Entwicklung des Gewahrseins bei:

"[...] a central aspect of Gestalt therapy is its open exploratory attitude that avoids imitations, mechanical assumptions or sterile repetitions and seeks the unique creative resolution of this unique situation for this unique person, in this relationships, in this field, at this moment in time" (Clarkson \& Mackewn 1993, S. X 2).

Menschen mit Depressionen sind manchmal blockiert auf verschiedenen Ebenen. Z.B. ist bei ihnen die sensorische, emotionale, kognitive Ebene und die Handlungsebene ausdrücklich gehemmt, also unterdrückt. Die Gestaltarbeit mit kreativen Medien bietet die Möglichkeit, auf unterschiedlichen Ebenen des menschlichen Erlebens verstummte 


\section{Arbeitskonzept für die Gestaltarbeit mit kreativen Medien}

innere Konflikte zu erkennen und zum Ausdruck zu bringen, indem die Förderung des "Gewahrseins“ im „Hier und Jetzt“ den Erlebnisprozess beim Menschen begleitet.

In diesem Sinne ist die Förderung des Gewahrseins des Hier und Jetzt sowohl für den Klienten als auch für den Therapeuten ein Hebel, damit die inneren Erfahrungen von Klienten mit Depressionen durch Gewahrsein anerkannt werden können. Die Anerkennung der inneren Erfahrungen seitens des Klienten ist der erste Schritt zur Heilung und kann auf der Seite des Therapeuten in der therapeutischen Beziehung stattfinden, aber der Therapeut muss den Klienten ermutigen, die innere Welt und ihre Wirkung zu bemerken, die Funktion der depressiven Symptome anzuerkennen und ihre Aussage als Symptom zu "hören“ und zuzulassen, was er braucht. Die Gestaltarbeit mit kreativen Medien unterstützt die Erlebnisse mit unterschiedlichen Kommunikationskanälen für die anschauliche Wahrnehmung der inneren Welt und der verleugneten inneren Erfahrungen, die von dem Klienten anerkannt werden müssen.

Dieser Arbeitsprozess ist nicht nur intellektuell, wie bei Interventionen bei der Kognitiv- und Verhaltenstherapie, sondern mit dem ganzen Leib des Klienten holistisch erfahrbar. Die inneren unassimilierten Erfahrungen von Klienten mit Depressionen müssen wahr- und ernstgenommen werden, da sie immer Kontaktunterbrechung zu sich selbst, zum anderen und zum Umfeld bringen, mit der Folge, dass depressive Bilder entstehen. Wenn man den Kontakt zu sich selbst, zum anderen oder zum Umweltfeld verliert, fühlt man sich einsam, da das menschliche Wesen Resonanz und Verbindungsgefühl unabdingbar braucht. Zunächst arbeiten die Klienten in diesem Konzept mit kreativen Medien nach dem Prinzip der „Förderung des Gewahrseins", so dass der Kontakt zum Selbst (besonders der eigenen Bedürfnisse), dann zum Anderen und zu den Lebensräumen in verschiedenen Dimensionen mit dem näheren Ziel der Problembewältigung und mit dem distalen Ziel der Stärkung der Selbstunterstützung stattfinden kann.

Wie erwähnt, ist Gewahrsein in der Gestaltherapie sowohl terminus a quo als auch terminus ad quem. Dies ist mit dem Fokus der „Klärungsperspektive“ (Grawe 1994) verbunden, was als ein wichtiges Wirkprinzip der Psychotherapie gilt. Dieser Fokus wird in der Gestalttherapie als "fokussierte Awareness" bezeichnet, damit ein bestimmtes Problem näher untersucht wird, so dass der Klient die Problemsituation versteht und seine fixierten Anpassungsmuster im Umgang mit der Situation wahrnimmt. Während der Gestaltarbeit zur Klärung dessen, was das Problem in einer Situation oder Beziehung ist, geht es insbesondere um die Bewusstheit dessen, was passiert (Inhalt), sowie um die bewusste Verfolgung des Prozesses, wie etwas genau 


\section{Arbeitskonzept für die Gestaltarbeit mit kreativen Medien}

passiert (Awareness of the awareness process) (vgl. Votmeier, 1994). Dieses prozessorientierte Vorgehen der Gestalttherapie und der emotionsorientierten Therapie (EFT) spielt eine fortführende Rolle in der Psychotherapie. Die „fokussierte Awareness" führt einerseits zur Strukturierung der Situation, in der die Ressource aktiviert wird, andererseits zur Problemaktualisierung und folglich zur Problembewältigung. Das „Wirkprinzip zur Förderung des Gewahrseins des Hier und Jetzt“ begleitet den Klienten sowie den Therapeuten von Anfang bis Ende in verschiedenen Phasen der Therapie. In dieser Arbeit werden kreative Medien insbesondere eingesetzt, um dem Klienten zu helfen, mit sich selbst nicht abstrakt sondern ganz konkret in Kontakt zu kommen, indem zunächst seine sensomotorische Aufmerksamkeit entwickelt wird und dann eine differenzierte Wahrnehmung der eigenen Bedürfnisse stattfinden kann.

\subsubsection{Phänomenologischer Vorgang}

Der phänomenologische Ansatz trägt zu aller Psychotherapie bei. In der Gestaltarbeit wird er auf zwei unterschiedlichen Weisen betont und reflektiert. Eine von ihnen wird zur Betonung und zur Vertiefung des Gewahrseins spezifiziert, so dass es (das Gewahrsein) die Besonderheit des therapeutischen Prozesses in der Gestaltarbeit beseelt und reich macht. Eine andere Art dieses Ansatzes ist die dialogische Beziehung Yontef (2007) unterscheidet vier Merkmale des dialogischen Kontakts in der Gestalttherapie:

(a) Presence (Präsenz): Präsenz erfordert vom Therapeuten wahrzunehmen, zu fühlen, zu verstehen, zu akzeptieren und Verantwortung für die Gefühle im therapeutischen Prozess zu übernehmen.

(b) Inclusion (Einbeziehung): Einbeziehung ist der Prozess des Therapeuten, in dem er sich gänzlich in die Perspektive des Klienten versetzt. Solche Haltung schafft für den Kleinten ein Gefühl der Sicherheit, sodass er sich nicht allein sondern beschützt und verstanden fühlt. In der Gestalttherapie ist der Fokus der Einbeziehung nicht nur auf dem, was der Klient erlebt, sondern auch auf dem Prozess der Experiences. Der Therapeut tritt respektvoll in die phänomenologische Welt des Klienten ein und erlebt ihn, wie er ist.

(c) Confirmation (Bestätigung): „Bestätigung" ist ein der „Einbeziehung“ zugehöriger Aspekt. „Bestätigung“ beinhaltet sowohl die gegenwärtige Realität als auch das, was der Klient werden kann. An einer existentiellen Begegnung, in der der Therapeut die Wirklichkeit der Existenz des 


\section{Arbeitskonzept für die Gestaltarbeit mit kreativen Medien}

Klienten erfährt, bestätigen sich Sein und Werden des Klienten.

(d) Commitment to "the between" (Verpflichtung zum Zwischen): Der Therapeut vertraut dem, was in der Interaktion zwischen Therapeut und Klient entsteht, und er bewundert das, was in der Interaktion zwischen Therapeut und Klienten entsteht. Er lernt von der phänomenologischen Exploration und der dialogischen Interaktion (vgl. S. 21).

Um die Welt des Klienten zu erreichen und um eine therapeutische Beziehung aufzubauen, sieht das Gestaltmodell phänomenologische Fokussierung, Experimente und dialogische Beziehung vor:

"The therapist's reality is not more valid or objective or true than the patient's. This is especially true since psychotherapy is centered on the patient, it is the patient's existence that is the reason for the therapy, and it is the patient that has the primary data. The patient's sense of self is as phenomenologically real and valid as the therapist's sense of the patient. Conversely, the patient's sense of the therapist in the therapist-patient interaction is as valid a phenomenological reality as the therapist's self-concept. This attitude is crucial for a truly relational therapy“ (Yontef 2002, S. 16-17).

In der Gestaltarbeit zielt die phänomenologische Fokussierung nicht nur auf den Klienten ab, sondern auch auf das Gestaltete, das der Klient im Gestaltungsprozess kreiert hat, und auf den Prozess.

Herman Schmitz (1989) beschreibt den phänomenologischen Prozess als dreischrittig: das deskriptive Stadium, das analytische Stadium und das kombinatorische Stadium. Im ersten Stadium nimmt der Therapeut das Phänomen, wie es ist, wahr. In diesem Stadium ist es wichtig, Phänomene und Interpretation möglichst zu trennen, da unsere Wahrnehmung von Phänomenen immer mit Vorurteilen und ungeprüften Interpretationen verbunden ist (Rahm et al., 1993). In der Gestaltarbeit mit aktivem Einsatz kreativer Medien nimmt der Therapeut das Phänomen des Klienten, das Gestaltete und den Gestaltungsprozess wahr, indem er seine Interpretation und seine Vorurteile ausklammert.

Im zweiten Stadium untersucht der Therapeut die Qualität und Bedeutung des Phänomens genauer. Es geht in diesem Stadium um die Erweiterung des Phänomenfeldes und um die Prägnanz des Phänomens. In der Gestaltarbeit fokussiert der Therapeut das prägnante Phänomen des Klienten oder des Prozesses des Klienten, 


\section{Arbeitskonzept für die Gestaltarbeit mit kreativen Medien}

z.B. Gestik, Mimik oder Haltung usw., mit dem Arbeitsprinzip des „Hier und Jetzt“, und er lässt den Klienten selbst das Phänomen bewusst, leiblich und anschaulich erleben, sodass der Klient sich oder die Beziehung mit seinen Lebensräumen wahrnimmt, sie versteht und kreativ an sein Leben anpasst. Während und nach der Gestaltung mit kreativen Medien entstehen auch Phänomene, die mit der Methode der Fokussierung prägnanter werden, damit der Klient sich mit seiner Fähigkeit zur Exzentrizität vom Gestalteten erforschen und auseinandersetzen kann.

Im dritten Stadium, dem „kombinatorischen Stadium“ geht es um die Sammlung von Phänomenen und das Herausarbeiten der latenten Struktur von Phänomenen. Die "phänomenologische Methode ist ein bewusstes systematisches Vorgehen zur Durchdringung der Wirklichkeit. Sie geht von den Phänomenen zu den Strukturen und erhält damit den Boden zur Erschließung von Lebensentwürfen" (Rahm et al. 1993). In der Gestaltarbeit mit kreativen Medien fördert der Therapeut das Erkennen der vermuteten Struktur der Phänomene, indem die Zusammenhänge zwischen den Phänomenen, der Lebensgeschichte und der aktuellen Situation der Lebensräume des Klienten herausgearbeitet werden. Für die spürbare und anschauliche Anregung beim Erkennen des Klienten haben der Gestaltungsprozess mit kreativen Medien und das Gestaltete ein enormes Potential (siehe. Kap. 3.5.).

\subsubsection{Prozess und Themen betreffende (bezogene) Prinzipien}

\subsubsection{Arbeitsverlauf nach Prozess und Themen}

Grawe (1995, 1997b, Grawe et al. 1994) untersucht aus zahlreichen empirischen Studien therapeutische Faktoren und konzipiert vier Wirkfaktoren: Problemaktualisierung, Problembewältigung, motivationale Klärung, Ressourcenaktivierung. Diese verschiedenen Wirkfaktoren sind als Abstraktionen eines einheitlichen und ganzheitlichen Veränderungsprozesses in dieser Gestaltarbeit miteinander verbunden bzw. gegenseitig beeinflusst und aktiviert. In der vorliegenden Arbeit werden diese Wirkprinzipien unter zwei Arbeitsschwerpunkten - erlebnisorientierte Exploration und aktiver Ausdruck - mit dem aktiven Einsatz kreativer Medien zur indikativen Prävention Depression bei Jugendlichen berücksichtigt. Der Arbeitsverlauf des Programms zur indikativen Prävention von Depression besteht aus drei Arbeitsphasen mit dem aktiven Einsatz kreativer Medien: die erlebnisorientierte Explorationsphase, die aktive Ausdrucksphase und die Integrationsphase.

„Therapie“ versteht man im Rahmen der Gestalttherapie als „Wachstumsprozess". 


\section{Arbeitskonzept für die Gestaltarbeit mit kreativen Medien}

Beim Wachstum wird das Problem, das den Klienten gerade belastet, gelöst, damit er mit neuen Einsichten sein Leben kreieren kann. In diesem Sinne wird Wachstumsprozess ersetzt durch den "schöpferischen Prozess", in dem das Leben ein Prozess von „Integration und Kreation“ ist. Hierbei hat der therapeutische Prozess mit kreativen Medien eine große Bedeutung, da das "Schöpferische“ in der Therapie prozesshaft und tatsächlich stattfindet, z.B. durch Bildliches, Tonfiguren und Improvisation mit Materialien. Hier liegt der Unterschied zwischen verbaler Therapie und der Therapie mit kreativen Medien. In der verbalen Therapie ist das Medium Sprache ein endgültiges Kommunikationsmittel zwischen Therapeut und Klient; dies hat in der Therapie mit kreativen Medien auch einen wichtigen Stellenwert, wirkt aber mit kreativen Medien zusammen.

In der Integrativen Therapie bietet das "tetradische System als Prozessmodell eine Strukurierungshilfe" (Petzold 1974; 1980); dies gilt auch für die Integrative Kunsttherapie und Kreativitätstherapie: Initialphase, Aktionsphase, Integrationsphase und Neuorientierungsphase. Dieser Prozess ist nicht linear zu verstehen, sondern er ist durch Kontinuität und Diskontinuität gekennzeichnet. Seine Interaktionen sind allerdings bei einem positiven Verlauf der Therapie der Tendenz nach progredient (vgl. Petzold \& Orth 1985). Richter (2003) stellt ein Modell in Verbindung des vierphasigen Prozessmodells der Integrativen Therapie und des Kreativitätsmodells Landauers für kreative Gestaltarbeit dar: Einlassungsphase, Experimentalphase, Arbeits- und Integrationsphase und Neuorientierungsphase.

In dieser Arbeit wird der Arbeitsprozess mit folgenden drei Arbeitsschritten durchgeführt.

a) 1. Schritt : Erlebnisorientierte Exploration

b) 2. Schritt : Aktiver Ausdruck

c) 3. Schritt: Integration

Im ersten Arbeitsschritt werden sowohl die Schwierigkeiten und Auffälligkeiten, als auch die Fähigkeiten und Stärken auf der Beziehungs- und Sozialebene im Lebenskontext, z.B. in der Familie und in der Schule, die für Jugendliche eine besondere Bedeutung haben, erhoben. In dieser Phase wird zunächst der Kontakt zum Selbst erforderlich, indem die Klienten mit ihrer Exzentrizitätsfähigkeit sich selbst im spezifischen Lebenskontext explorieren, sodass sie das Selbstbild, die Kontaktqualität mit sich selbst und mit anderen und das gesamte Bild im Lebenskontext überschauen

können. Hier gilt Auffälligkeit als Botschaft und Anfrage an Veränderungsmöglichkeiten (Rahm 1997) oder als die momentan bestmögliche 


\section{Arbeitskonzept für die Gestaltarbeit mit kreativen Medien}

Bewältigung (Pauls 2007) einer Belastung bzw. Krise des Jugendlichen. Hier zielt die Arbeit besonders auf die Aktivierung der Ressourcen und auf die Aktualisierung der Probleme bei Klienten mit der Hilfe von Malen ab.

Im zweiten Schritt werden die aktualisierten Probleme mit aktivierten Ressourcen ausgedrückt. In diesem Schritt gestalten die Teilnehmer noch mithilfe kreativer Medien wie Ton und Malmaterialien das, was über die Themen "Schatten des Selbst", „unterbrochene Prozesse oder Gefühle“ und „Konfliktssituation“ in bestimmten Lebensräumen symbolisiert wird. Sie arbeiten mit dem dargestellteten Produkt, das sowohl im ersten Arbeitsabschnitt als auch im zweiten Abschnitt gestaltet wurde, anstatt mit dem „Stuhls“. Beim aktiven Ausdruck bewältigen die Klienten die aktuellen Probleme. In diesem Abschnitt wird die Technik der Identifikation mit dem gestalteten Produkt, der Dialog mit dem gestalteten Produkt und (eventuell) das Rollenspiel genutzt, damit eine anschauliche Begegnung mit dem Produkt stattfindet.

Im dritten Schritt arbeiten Teilnehmer an der Integration dessen, was sie in der Gruppenarbeit bis jetzt erlebt haben. Sie schauen sich genau an, ob noch etwas und was noch als Rest übrig bleibt und haben dann die Chance weiterzuarbeiten. In diesem Schritt schauen sie auf ihren Weg zurück und lernen, den Kontakt nachklingen zu lassen und Abschied $\mathrm{zu}$ nehmen. Die Erklärungen für jeden Schritt gibt es im Folgenden.

\subsubsection{Problemaktualisierung und Ressourcenaktivierung im ersten Schritt}

Wichtig ist im Bereich der Psychotherapie oder der psychologischen Beratung der Trend zur Problemspezifizierung und zu einem differentiellen Vorgehen. Darüber hinaus sind die Ressourcen des Klienten aber wahrscheinlich wichtiger als seine Probleme hinsichtlich der Frage, wie die Probleme am besten gelöst werden können. Das Ressourcenkonzept ist nicht neu im Bereich der Psychotherapie und der Beratung, z.B. bei der Familientherapie, der Hypnotherapie, der lösungsorientierten Therapie, der Organisationsentwicklung, der Pädagogik, der Systemberatung etc. Die funktionale Rolle der Ressourcenaktivierung und die Bedeutung für die Herbeiführung therapeutischer Veränderungen sind von Grawe empirisch und theoretisch ausgearbeitet worden (Grawe 1999, S. 63ff).

In der erlebnisorientierten Explorationsphase beschäftigen sich der Klient und der Therapeut nicht nur mit der Erfassung der Probleme, sondern auch mit der Entdeckung und Aktivierung der Ressourcen des Klienten. Dafür stehen die Methoden bzw. Techniken der kreativen Medien zur Verfügung: In dieser Arbeit werden sie auf 


\section{Arbeitskonzept für die Gestaltarbeit mit kreativen Medien}

den drei Ebenen Selbst, Lebenskontext, sowie Schule und Familie zur Diagnostik und Informationserhebung für die Problemaktualisierung bzw. die Ressourcenaktivierung im Sinne der Saluto-Pathogenese verwandt. Petzold (1992) stellt vier diagnostische Grundfragen: a) Was funktioniert und muss erhalten werden? b) was ist beschädigt, bzw. gestört in der Funktion? c) was fehlt und muss bereitgestellt werden? d) welche Ressourcen sind ungenutzt und können entwickelt und erschlossen werden? Insbesondere hat die Ressourcenaktivierung am Anfang der Gestaltarbeit bei depressiven Jugendlichen einen wichtigen Stellenwert und setzt sich bis zum Ende der Arbeit fort.

Denn depressive Jugendlichen können ihre Ressourcen oft nicht erkennen und ihre Grundbedürfnisse nicht unter Nutzung eigener Ressourcen befriedigen. In der erlebnisorientierten Explorationsphase erfährt der Klient in der Arbeit mit kreativen Medien eine anschauliche Sicht auf seinen Lebenskontext, also Schule oder Familie, den Ort, wo er als „Mitmensch“ andere und sein Lebensfeld beeinflusst und von ihnen beeinflusst wird. Daher nimmt er die Probleme in Verbindung mit seinem Lebensfeld wahr. Darüber hinaus ist es auch unabdingbar, seine Ressourcen, Kräfte und Resilienzen zu entdecken, $\mathrm{zu}$ sehen und $\mathrm{zu}$ aktivieren. Die Balance beider Foki, nämlich von Problemen und Ressourcen, zu halten ist in der Arbeit für den Therapeuten immer eine wichtige Aufgabe. Wie er die Ressourcen, die Kräfte, das Coping und die Resilienzen seines Klienten sieht oder ob er sie überhaupt sehen kann, ist ein Bestandteil der gelungenen therapeutischen Beziehung, die zum Therapieerfolg enorm beiträgt. Die Ergebnisse der Studie von Grawe et al. (1998) bestätigen immer wieder in einem hohen Ausmaß die überragende Wichtigkeit der Ressourcenaktivierung für ein gutes Therapieergebnis in wechselnden Kontexten, für unterschiedliche Arten von Therapien, bei verschiedener Erfolgkriterien. Wenn der Therapeut die Ressourcen seines Klienten nicht vernachlässigt, sondern dazu ermutigt, sie $\mathrm{zu}$ entdecken und $\mathrm{zu}$ entfalten, dann wird nicht nur aktives Engagement des Klienten, sondern auch Verantwortung für sich selbst und die Therapie erhöht. Natürlich hat der Klient in dieser Kooperation mit dem Therapeuten, die „Korrespondenz" genannt wird, die Möglichkeit, von der Haltung des Therapeuten zu lernen, um in sich seine eigenen Kräfte, die wegen bisheriger Schwierigkeiten Überwindung kosten, zu spüren und zu aktivieren.

Dies ermöglicht dem Klienten wiederum bei der Ressourcenentdeckung und -aktivierung das Gefühl, selbst kompetent $\mathrm{zu}$ sein. Bei der Exploration zur Ressourcenaktivierung müssen die Ressourcen immer konkret in der Kooperation zwischen Klienten und Therapeuten angezapft werden. Das heißt, dass der Therapeut 


\section{Arbeitskonzept für die Gestaltarbeit mit kreativen Medien}

seinen Klienten nicht zwingen darf, damit der Klient Gelegenheit bekommt, seine Stärken und positiven Intentionen als eigene zu bestätigen und seine Selbstwert erhöhenden Wahrnehmungen zu erhalten. Diese Arbeit hilft dem Klienten darüber hinaus, deutlich motiviert zu sein.

Depressive sind unfähig, mit den eigenen Stärken in Kontakt zu kommen, haben ein negatives Selbstbild und sind kaum zielstrebig. Sie ziehen sich zurück, da sie einfach ihren Mut und ihre Lust verloren haben, sowohl ihre Bedürfnisse als auch ihre vitalen Lebensenergien zu spüren. Wenn die Therapie nur Probleme des Klienten behandelt, spiegelt sich der Klient immer in seinen schwachen Seiten wider, ohne sich davon trennen zu können, was momentan problematisch ist. „Ressourcenaktivierung ist ein pervasives Wirkprinzip" (Grawe 1999, S. 72). Sie setzt eine ressourcenorientierte Wahrnehmungs- und Denkweise auf Seiten des Therapeuten voraus. Deshalb ist die Haltung des Therapeuten sehr wichtig. Da sie sich durch das ganze Therapiegeschehen hindurchzieht, reicht es nicht, die Herstellung eines ressourcenorientierten Settings in Kursen $\mathrm{zu}$ üben, sondern Ressourcenaktivierung muss darüber hinaus auch in unstrukturierten Situationen immer wieder reflektiert und geübt werden (vgl. ebd.).

„Nutzbare Ressourcen sind alle im ,Ressourcenreservoir eines Systems (Person, Gruppe, Organisation) vorhanden“ (Petzold 1997, S. 447). Um diese nutzbaren Ressourcen wahrzunehmen, $\mathrm{zu}$ erkennen und $\mathrm{zu}$ bewerten, werden zielstrebige Interventionen mit kreativen Medien bezüglich eines bestimmten Lebenskontexts von Klienten berücksichtigt. In dieser Arbeit werden zur Ressourcenaktivierung sowie zur Problemaktualisierung bestimmte Methoden und Techniken, z.B. „Familie in Tieren“ und „das Schulpanorama“, angewandt. Die beiden Techniken können mit verschiedenen Variationen zur Erhebung der Probleme oder der Ressourcen in der Therapie eingesetzt werden. In dieser Arbeit können eine Menge Informationen zunächst durch Malen mit den Techniken zutage gefördert werden, einerseits wo sich der Teilnehmer in seinem Lebenskontext befindet und wie er sich fühlt, andererseits was er hat, braucht und sich wünscht. Folglich kann der Klient durch die Arbeit mit den Techniken seine Probleme und Ressourcen nicht nur explorieren, sondern kann sie auch während der Bearbeitung mittels verschiedener Kommunikationskanäle, z.B. Malen, Schreiben, Bewegen etc., erleben, sodass er zur Bewältigung seiner aktuellen Probleme seine Ressourcen verfügbar machen kann. Seine Probleme sind deutlich und nicht unterschwellig, und seine Ressourcen, die mit seinen Problemen durcheinandergemischt und unverfügbar geworden sind, weil ihre Macht verloren gegangen ist, werden wahrgenommen und aktiviert, sodass sich der Teilnehmer für die Problembewältigung engagieren kann. 


\section{Arbeitskonzept für die Gestaltarbeit mit kreativen Medien}

In der Gestaltherapie zielt der Fokus auf die Ressourcenaktivierung ab, auf Support (Resnick 1990), z.B. Intellekt, Körper, Finanzen, etc. Besonders hat der "SelfSupport" (Selbstunterstützung) einen wichtigen Stellenwert in der Gestalttherapie. Resnick (1990) meint, dass der "Support“ (Unterstützung) in etwa dem analytischen Konzept von „Ich-Stärke“ entspricht und der Selbstregulation dient (a.a.O. S. 104ff). Wenn man seinen Zustand im Moment identifizieren kann, kann man seine Fähigkeit zur Bedürfnisbefriedigung, also zur Selbstregulation weiter aktivieren. Wenn einem diese Fähigkeit zur Selbstunterstützung fehlt, scheitert man am Kontakt zu sich selbst, mit anderen oder mit der Umwelt, sodass man wegen der „unerledigten Bedürfnisse“ nicht frei wird. Aus diesem Grund hat die Gestalttherapie mit der Verbesserung der Selbstunterstützung zu tun.

Deshalb ist es in der Gestalttherapie wichtig, den eigenen Zustand zu identifizieren, zunächst wahrzunehmen, was der Organismus momentan braucht. Das bedeutet, dass das Gewahrsein den Zustand der Ressourcenaktivierung voraussetzt. Deshalb hat Perls gesagt, "Gewahrsein allein kann heilsam sein“. Dieser Zusammenhang lässt sich nach der Theorie des „Paradoxes der Veränderung“ (Beisser 1980) erklären. Wenn ein Klient er selbst sein kann, ohne sich zu bemühen jemand anders zu werden, wird er sich verändern. Es ist klar, dass in der Gestalttherapie Ressourcen und ihre Aktivierung wichtig sind, indem der Therapeut durch verbessertes Gewahrsein des Klienten auf verschiedene Arten, z.B. durch Interventionen bezüglich der Körperhaltung oder wahrnehmung, seine Ressourcen zu aktivieren hilft. Dennoch hat die Gestalttherapie noch Bedarf an der Ausarbeitung konzeptueller und methodischer Ansätze, um gezielter an dem Fokus zur Ressourcenaktivierung zu arbeiten (vgl. Votsmeier 1994).

\subsubsection{Problembewältigung, Auflösung der Konflikte und unterbrochene Prozesse oder Gefühle im zweiten Schritt}

In zweitem Arbeitsabschnitt „Aktiver Ausdruck“ arbeitet der Teilnehmer mit seinem gestalteten Produkt, das er während der Gestaltung mit kreativen Medien geschaffen hat. Was in erstem Arbeitsschritt aktiviert und aktualisiert wird, mündet in dieser Phase in die Problembewältigung ein. Selbst bei Problemaktualisierung reicht die Ressourcenaktivierung nicht aus, sondern sollte durch Bewältigungsverhalten (coping) und/oder schöpferische Gestaltung, also kreative Anpassung realisiert werden. Dafür hat die Gestaltarbeit mit Hilfe kreativer Medien gute Möglichkeiten, sie zu realisieren, nämlich beim „aktiven Ausdruck“.

Ein zentrales Ziel in der Gestalttherapie ist die Wiederbelebung und Verfeinerung des 


\section{Arbeitskonzept für die Gestaltarbeit mit kreativen Medien}

emotionalen Sensoriums durch aufmerksameres Gewahrsein. Da Gefühle selbst in der Gestalttherapie eine kathartische Wirkung haben, ein Mittel des Erkennens sind, ist die Arbeit zur Verfeinerung der Emotionen und der emotionalen Empfindsamkeit sehr fundamental. Bei depressiven Klienten ist es insbesondere notwendig, die unterdrückten Gefühle deutlich zu empfinden und wieder zu beleben, damit der emotionale Ausdruck stimmig und authentisch wird (vgl. Dreitzel 1995).

Im ersten Arbeitsschritt zur erlebnisfördernden Exploration werden Probleme aktualisiert und es wird vom Klienten erkannt, was und wo die Probleme sind. Dabei hat der Klient seine Stärke empfunden. Durch den Ausdruck der aktuellen Gefühle erleben depressive Klienten Erleichterung. In der Gestaltarbeit mit aktivem Einsatz kreativer Medien arbeiten die Klienten beim aktiven Ausdruck mit ihrem gestalteten Produkt, das sie als Symbol der unterbrochenen Prozesse oder Gefühle in Bezug auf bestimmte Personen und der Selbstspaltung in Bezug auf Selbstkritik oder Gefühlsblockierung aus Ton oder in Form von Malen gestaltet haben. Nach der Gestaltung arbeiten depressive Teilnehmer je nach aktuellen Themen an den "Anzeichen“ (Marker) der „Selbstbewertungsspaltung“, der „selbstunterbrechenden Spaltung“, der "unterbrochenen Prozesse oder der schmerzhaften Emotion“ mit dem Produkt, das ein betimmtes Thema symbolisiert. Bei dem Thema „Selbstbewertungsspaltung“ (Selbstkritik oder Zerrissenheit) drücken die Teilnehmer mit dem Gestalteten ihre Gegenseite aus, statt der Zwei-Stuhl-Technik, die üblicherweise in der Gestalttherapie genutzt wird, damit sie ihre Selbstspaltung und Probleme bei Selbstkritik überwinden. Bei dem Thema „selbstunterbrechende Spaltung" (Gefühlsblockierung oder Resignation) arbeiten die Klienten mit dem Gestalteten statt der Zwei-Stuhl-Technik, um ihre Gefühle zu inszenieren, damit sie durch den aktiven Ausdruck Selbstwertgefühl und Stärke bekommen. Es geht hier um Integration.

„Die grundlegende Lehre der Gestalttherapie ist die der Wesensdifferenzierung und der Integration. Die Differenzierung als solche führt zu Polaritäten. Als Dualitäten werden diese Polaritäten leicht in Streit kommen und sich gegenseitig paralysieren. Indem wir gegensätzliche Züge integrieren, machen wir die Menschen wieder ganz und heil. Zum Beispiel Schwäche und tyrannisches Verhalten integrieren sich als ruhige Festigkeit“" (Perls 1966, in Petzold 1980, S. 155).

Bei dem Thema „unterbrochene Prozesse oder dauerhafte negative Gefühle gegenüber 


\section{Arbeitskonzept für die Gestaltarbeit mit kreativen Medien}

bestimmten anderen Menschen" arbeitet der Klient mit dem Gestalteten, das meistens das Gesicht von bestimmten Personen darstellt. Dieses Gestaltete evoziert ganz konkret ihre aktuellen Gefühle und Themen, deshalb ist der aktive Ausdruck für Klienten ein sicherer Arbeitsweg, auf dem sie ihre Gefühle nicht verlieren und nicht abstrakt phantasieren, sodass sie ihre unterbrochenen Prozesse oder Gefühle in Bezug auf bestimmte Personen abschließen können. Bei dem aktiven Ausdruck unterstützt der Therapeut die aktiven Experimente und Variationen des Ausdrucks des Klienten und ermutigt ihn, neues Verhalten oder neuen Ausdruck auszuprobieren, sodass er in einem geschützten Rahmen ein Risiko eingehen kann. Da das Gestaltete schon die innere Landschaft hinsichtlich des bestehenden Problems relevant hervorgehoben hat, können unbewusste innere Prozesse wie Gefühle, Gedanken oder Schemata relativ leicht aktiviert werden. Durch den aktiven Ausdruck mit dem Gestalteten können sogenannte korrigierende Erfahrungen gemacht werden, wodurch Alternativen zu fehlangepassten Verhaltens-, Beziehungs- und Einstellungsmustern verfügbar werden (vgl. Votsmeier 1994).

\subsubsection{Stärkung zur Selbstunterstützung und Gewinnung eigenen Lebenssinns im dritten Schritt}

Solche Problembewältigung führt letztendlich zur Selbstunterstützung. SelbstUnterstützung beinhaltet "Selbst-Kenntnis“ (Self-Knowledge) und "SelbstAkzeptanz" (Self-Acceptance) (Yontef, 1993). Selstunterstützung bezieht sich auf die Fähigkeit, die Verantwortung für sich selbst und für die Zufriedenheit hinsichtlich der Bedürfnisse des Selbst und zur klaren Wahlfreiheit bezüglich der Bedürfnisbefriedigung zu finden. Das ist ein wichtiges Ziel der Gestaltarbeit nicht nur für Jugendliche mit Depression, sondern überhaupt. Problemaktualisierung und Ressourcenaktivierung zielen auf Problembewältigung ab, die immer in diesem Sinne Selbst-Regulation und Selbst-Unterstützung fördert. Dass Selbst-Unterstützung und ihre Funktion gelernt und erlebt werden, ist ein schwerwiegendes Entwicklungsthema im Jugendalter. Eine Balance zwischen Abhängigkeit und Unabhängigkeit als aktuelle Entwicklungsaufgabe zu finden, führt zum Gefühl, kompetent sein zu können, so dass Jugendliche ihr Leben selbst zu organisieren vermögen. Erwachsen zu werden beruht darauf, dass man mehr Selbst-Unterstützung als Umwelt-Unterstützung realisierbar macht. In der Gestaltarbeit findet man seinen eigenen Lebenssinn, indem man sich mit dem Gestalteten von sich mit Exzentritätsfähigkeit begegnet und den Zusammenhang zwischen sich und seinen Lebensräumen erkennt und die eigenen Schmerzen und 


\section{Arbeitskonzept für die Gestaltarbeit mit kreativen Medien}

Bedürfnisse fühlt.

„Die Konstruktion persönlicher Bedeutungen besteht demnach in einem Prozess unablässiger Synthese von Information aus unterschiedlichsten Quellen und deren bewusster Symbolisierung, mit dem Ziel, eine subjektive Wirklichkeit zu schaffen. Dieser Prozess dialektischer Konstruktion erfordert gleichzeitig die bewusste Wahrnehmung körperlich empfundenen Erlebens und die Schaffung einer spezifischen augenblicklichen Repräsentation desselben. Durch diesen dialektischen Prozess des Symbolisierens von Erleben im Gewahrsein entstehen neue Sichtweisen des Selbst und der Wirklichkeit“ (Greenberg et al. 2003. S. 99).

Aus den gemeinsamen Erforschungen zwischen Therapeut und Klient sowie zwischen Klient und seinem Gestalteten durch kreative Medien können sich viele verschiedene Variationen zur Lösung und ein eigener Lebenssinn ergeben, sodass die Arbeit mit wechselnden Feld-Konfigurationen eine ungeheuere Variationsbreite erlaubt.

\subsection{Therapeutische Phasen der Arbeit mit kreativen Medien}

Im Folgenden wird das von der Verfasserin erarbeitete Phasenmodell und seine Erprobung im Rahmen von Gestaltarbeit vorgestellt. Die Therapiephasen werden je nach Setting bestimmt, z.B. Gruppen- oder Einzelarbeit, stationäre oder ambulante, psychotherapeutische, Sozial- oder Beratungsstelle, Fokal- oder langfristige Arbeit, usw. Der Schwerpunkt liegt hier durch die Aktionen des Klienten auf der Arbeit mit kreativen Medien und in der Wechselwirkung kreativer Medien. Die Gestaltarbeit mittels des aktiven Einsatzes kreativer Medien wird anders als die ohne sie durchgeführt. In der Gestaltarbeit mit kreativen Medien wird die "Gestaltungsphase“, die es in der normalen Gestaltarbeit nicht gibt, hinzugefügt. Der Einsatz von kreativen Medien ist in der Integrativen Therapie und Supervision eine Intervention, der sehr viel Bedeutung beigemessen wird.

Mit dem Einsatz kreativer Medien entsteht etwas Neues, das mit und wegen kreativer Medien evoziert und kreiert wird. Dies ist nicht nur ein Produkt, sondern auch ein Prozess. Deshalb ist es erforderlich, dass der Therapeut oder Berater fundiertes Wissen über die Arbeit mit kreativen Medien und die Bereitschaft und Sensibilität für das „entdeckende Gespräch“ im Anschluss an den schöpferischen Prozess, der während und nach der Gestaltung mit kreativen Medien auf Seiten des Klienten entsteht, 


\section{Arbeitskonzept für die Gestaltarbeit mit kreativen Medien}

verfügt.

Kreative Medien sprechen „drei Realitätsebenen“ (Petzold 1998a, S. 307) an: 1. die Ebene des Realen: die der real beobachtbaren Wirklichkeit. 2. die Ebene des Imaginären: die gedanklichen bzw. vorstellungsmäßigen Repräsentationen von Wirklichkeit. 3. die Ebene des Symbolischen: die der symbolisch verdichteten Wirklichkeit. Während und nach der Gestaltung mit kreativen Medien entsteht prägnant ein wichtiges Thema des Klienten und der Therapeut oder Berater muss ihm dabei helfen, es ins Bewusstsein kommen zu lassen (Awarenessförderung). Dieser Erkenntnisprozess führt zum Wachstumsprozess. Normalerweise wird die therapeutische Arbeit mit kreativen Medien in folgenden Schritten aufgebaut (vgl. Nitsch-Berg \& Kühn 2000): a) die Evozierung der Hintergrunderfahrungen und Hinlenkung der Awareness auf die körperlichen Empfindungen und Wahrnehmungen, b) die Verdichtung der erinnerten Erlebnisse und Wahrnehmungen, c) die Gestaltung und Projizierung der verdichteten Wahrnehmungen mit kreativen Medien, d) die Wahrnehmung und Verarbeitung der Produkte.

Nun geht man davon aus, dass der Klient seine Probleme oder Symptome schon berichtet und eine therapeutische Allianz geschlossen hat. Über die Arbeitsweise, die theoretische und therapeutische Einrichtung wurde er informiert, und er erklärt sich einverstanden, $\mathrm{zu}$ einem guten Arbeitsbündnis beizutragen, sodass sich eine therapeutische Beziehung entwickeln kann. Natürlich wird vorausgesetzt, dass ein wachstumsfördernder Raum für den Klienten etabliert wird, wo der Klient Lust auf Experimente, Selbstexploration und die Möglichkeit zum freien Ausdruck und zur Regression haben kann, also zur Schaffung der Perls'schen „sicheren Krise“, das, was Winnicott (1973) mit „Facilitating Environment”, "potential Space“ meint. Dabei ist es wichtig, dass es nicht darum geht, ein künstlerisch wertvolles Werk zu schaffen, sondern Empfindungen, innere Bilder und Erfahrungen und Gedanken spielerisch, fließend und spontan zu erleben und auszudrücken, indem man mittels des Einsatzes kreativer Medien Kontakt zum Selbst, mit Anderen und mit der Umwelt vitalisiert. Mit dieser Berücksichtigung stelle ich hier das Phasenmodell in der Gestaltarbeit mittels des Einsatzes kreativer Medien dar. Das Modell ist ein Entwurf, um den Verlauf in jeder Phase deutlich zu erkennen. Bei keiner Phase gibt es radikale Trennung von der nächsten Phase, und der Verlauf hängt, wie immer, von dem therapeutischen Kontext ab. Hierbei läuft die Arbeit in jeder Sitzung vierphasig ab:
a) Aufbau
b) Gestaltung
c) Intensität: Ausklingen
lassen/Begegnung Zusammenführung und Eröffnung neuer Wachstumsdimensionen. 


\section{Arbeitskonzept für die Gestaltarbeit mit kreativen Medien}

\subsubsection{Phase 1: Der Aufbau}

In der ersten Phase wird die Arbeit mit einem „Warm-Up" oder einer Einstimmung begonnen. Die Hintergrunderfahrungen von gestern oder vergangener Woche werden im „Hier und Jetzt" evoziert. Dabei wird die Aufmerksamkeit auf die körperlichen Empfindungen und Wahrnehmungen gelenkt. Die Einstimmung ist „das Vehikel, über das alte, zum Teil verdrängte Szenen aufsteigen können, gegenwärtig gesetzt werden, ins Bewusstsein treten“ (Orth 1995. S. 319).

Die Ziele in dieser Phase sind für den Klienten erstens: Aufbau eines freien und sicheren wachstumsfördernden Raums und Klimas, zweitens eine behutsame Einstimmung zur Erreichung möglichst hoher Selbstzentrierung mit dem Ziel großen Gewahrseins. Am Anfang dieser Phase werden verschiedene Empfindungen und Eindrücke vermischt, die wahrgenommen werden oder noch nicht wahrgenommen werden, also unterschwellig bleiben. Der Klient sagt oder denkt z.B.: „Da gibt es was, aber ich weiß nicht, was es ist." Die Sache ist noch nicht prägnant. Hierzu wird der Klient aufgefordert, auf Gefühle, Empfindungen und die Körperhaltung bewusst zu achten und sich zu konzentrieren, Fantasien und Bilder auftauchen $\mathrm{zu}$ lassen und Bewegungs- und Ausdrucksimpulsen nachzugeben.

Manchmal können in dieser Phase gelenkte Imaginationen, Geschichten und Bewegungsübungen mit Musik eingesetzt werden. Die Impulse, mit denen sich der Klient gerade beschäftigt, können verstärkt werden, um seine Wahrnehmung zu sensibilisieren. Verstärkte Impulse können z.B. ein lautes Gähnen, körperlicher Ausdruck oder eine ruhige Meditationshaltung sein. Für die thematische Konkretisierung helfen solche Übungen dem Klienten. Sowohl in der Gruppen- als auch in der Einzelarbeit kann der Therapeut ein Thema benennen. Solch ein thematisches Angebot des Therapeuten kann manchmal überfordernd, aber auch oft hilfreich und notwendig sein, z.B. bei der Arbeit im Rahmen einer Kurzzeitgruppentherapie.

In dieser Phase lernt der Klient die eingesetzten Medien kennen; dabei können Fragen bzw. Gefühle aus früheren Erfahrungen auftauchen. Welche Medien verwendet werden, sollte sorgsam bedacht werden. Die mit den Medien in Zusammenhang gebrachten Fragen und Gefühle sollten behutsam behandelt werden. Manchmal hat der Klient Widerstand gegen und Unsicherheit bei der Gestaltung oder dem Ausdruck mit den bzw. durch die Medien aufgrund schlechter Erfahrungen und falsch ausgebildeter Kriterien in der Schule und der Gesellschaft. Dafür muss der Therapeut die Sitzung mit sicherem Klima und klar und wohlwollend einleiten. 


\section{Arbeitskonzept für die Gestaltarbeit mit kreativen Medien}

Es ist besonders in dieser Phase wichtig, dass der Klient sorgfältig in eine spielerische, kreative Welt eingeladen wird. Wenn der Klient in diese Welt zum Ausdruck und zur Gestaltung mit kreativen Medien eintritt, bedeutet das, dass der Klient wagt, in die neue Situation hineinzugehen und dem Prozess vertraut, damit er in der therapeutischen, kreativen und wachstumsfördernden Welle mitschwimmen kann. Dafür muss der Therapeut in dieser Phase einen sicheren Raum aufbauen, damit Verdrängtes, Tabuisiertes, Unbehagliches, Bedrohliches, Zorn und Schmerz vom Klienten möglichst mit geringer Mühe zum Ausdruck gebracht werden können.

\subsubsection{Phase 2: Die Gestaltung}

Der Prozess der Gestaltung ist der Ausdruck in der Gegenwart durch Hände, Augen und dem ganzen Leib. Was im Hintergrund geblieben war und endlich wahrgenommen wird, wird vom Klienten weiter exploriert und erinnert (vgl. FrohneHagemann 1999). In dieser Phase ist es erforderlich zuzulassen, dass die Figur aus dem Hintergrund auftaucht, Themen und Probleme konkretisiert werden, die mit Medien zum Ausdruck gebracht werden. Dabei ermutigt der Therapeut den Klienten, dem zu vertrauen, was gerade in ihm auftaucht und geschieht, und er unterstützt sein expressives Selbst, damit der Klient die falschen Kriterien und Klischees, die manchmal in der Schule gebildet wurden, überwunden werden und ihn möglichst wenig am Ausdruck hindern können. In dieser Phase sollten Zorn und Angst akzeptiert werden, statt sie zu konfrontieren oder abzuweisen, da solche Gefühle und Widerstände ein Anfang der Therapie sind.

Zum Ausdruck fordert der Therapeut oder Berater den Klienten auf, sich auf Gefühle und körperliche Empfindungen, z.B. das Atmen oder die Haltung zu konzentrieren und es nicht schön machen zu müssen, um anderen zu gefallen, sondern um stimmig zu machen, was er in sich hat und was auftaucht, sodass er die Energie aus sich zur Schöpfung fließend zulässt und folglich eine prägnante Figur von ihm spürbar, sichtbar geboren wird. In dieser Phase werden Kreativitätsvermögen, schöpferische Aktivität mit dem Prinzip des „Hier und Jetzt“ aktiviert und gefördert.

Da die Arbeit nicht allein produktorientiert für den Klienten ist, sondern auch prozessorientiert (vgl. Orth \& Petzold, 1990b), können Interventionen während des Gestaltungsprozesses gegeben werden, damit der Klient anhand seiner körperlichen Haltung, der Atmung und der Mimik deutlich wahrnehmen kann, was gerade vor sich geht, und er dann die Qualität des Erspürten, des Empfundenen und des Gefühlten an das Produkt weitergeben kann. Allen Medien ist hier ein projektives Potential eigen. In 


\section{Arbeitskonzept für die Gestaltarbeit mit kreativen Medien}

ihnen und durch sie können sich die Strebungen des Unbewussten und dessen, was sich aus dem Strom im Körper als Impuls und Regung vermittelt und was nach Ausdruck sucht, verkörpern (vgl. Orth, 1995, S.320-321). Mit den Interventionen in dieser Phase wird der Klient ermutigt, die in seinem Inneren auftauchenden archaischen Gestalten anzunehmen und prägnant werden zu lassen. Dieser Prozess kann heilende Kräfte, eine Katharsis freisetzen und für den Klienten eine wichtige Zeit sein, um sich seiner Innenwelt zu widmen.

Perls weist darauf hin, dass Kontakt kein angestrengtes Bemühen ist, sondern ein Geschehenlassen. Im Gestaltansatz geht es um die Entwicklung von Vertrauen in das, was geschieht und sich entwickelt, und nicht um den Aufbau von Kontrolle (vgl. Franck 1997). Der Organismus strebt danach, möglichst prägnante Gestalten zu verwirklichen. Der Hintergrund ist das Reservoir, aus dem eine Figur prägnant wird und endlich in der Welt gestaltet wird. Das gestaltete Produkt mit kreativen Medien ist nicht anders als ein Kunstwerk.

In dieser Phase versucht der Kleint, einfach herauszulassen, und vertraut dem, was „Hier und Jetzt" wahrgenommen wird und es geschieht. Dabei gibt es für den Klienten die Lernchance, sich $\mathrm{zu}$ spüren, wahrzunehmen und $\mathrm{zu}$ zeigen, sich auf ungewöhnliche Weise zum Ausdruck zu bringen, und die Möglichkeit, Spaß zu haben, spontan und authentisch zu sein, verinnerlichte und ausgebildete Barrieren aus der Schule zu überschreiten, sein eingeschränktes „expressives Selbst“, seine „Ich-Grenzen zum Ausdruck“ (Polster \& Poster 1975) in Schwung zu bringen und zu entfalten.

Die kreative Schöpfung ist ein Aufschrei der seelischen Qual, ein Überschreiten von Grenzen und ein Bejahen vom Leben hinter dem Leben, was unseren Mut kostet. „Der Mensch, der es wagt, schöpferisch zu sein, Grenzen zu überschreiten, hat nicht nur an einem Wunder teil, sondern kommt auch dahin zu erkennen, dass er in seinem Prozess des Seins ein Wunder ist" (Zinker 1982, S. 13).

Während der Gestaltung eines Produkts kann man sich im „schöpferischen Indifferenz- oder Nullpunkt" (Friedlaender, 1918) befinden, was Perls, Hefferline und Goodman „mittlerer Modus“ (Perls et al. 1991, S. 29 und 165) genannt haben.

„Der Künstler ist dessen gewahr, was er tut - wenn es getan ist, kann er sich jeden einzelnen Schritt zeigen; er ist sich seiner Arbeit nicht unbewusst, aber er arbeitet auch nicht allein mit bewusster Planung. Sein Gewahrsein ist in einer Art mittlerem Modus, weder aktiv noch passiv. Das Gewahrsein übernimmt die Bedingungen, es tut die Arbeit, und es wächst der Lösung entgegen“ (Perls et al., 1991, S. 29). 


\section{Arbeitskonzept für die Gestaltarbeit mit kreativen Medien}

Der Begriff „,schöpferische Indifferenz" wird in der Gestalttherapie als Ausdruck dafür gebraucht, dass man „weder krampfhaft versucht, völlige Kontrolle über eine Situation zu erlangen, noch passiv mit sich geschehen lässt, was immer passiert" (Blankertz \& Doubrawa 2005, S. 189). Das bedeutet, dass der Klient in diesem Moment von dem Gegenstand zum Gestalteten, z.B. ein inneres Bild oder das gerade aufgetauchte Gefühl, nicht getrennt, sondern reflexiv verbunden ist.

\subsubsection{Phase 3: Die Intensität}

Das gestaltete Produkt mit kreativen Medien ist nicht beliebig entstanden, sondern ist ein notwendiger und einmaliger Ausdruck vom Bedürfnis des Organismus nach dem „Prinzip des Feldes" (Lewin 1963), „der Prägnanz" (Köhler 1968) oder der "Gestalthöhe“ (von Ehrensfels 1916). Das Selbst ist eine Gestalt, die als Ergebnis der Suche nach der „guten Gestalt“ (Köhler 1968) gekennzeichnet ist. Das Selbst hinterlässt für den Organismus einen Eindruck. Das gestaltete Produkt selbst ist ein Ausdruck, sodass es für das Gegenüber einen Eindruck produziert, und besonders für den Gestalter eine Beziehung oder eine Bedeutung zwischen ihm (dem Produkt) und ihm (dem Gestalter) erfordert. Das bedeutet hier, dass es keine Spaltung zwischen dem Gestalter und der Gestalt gibt, sondern dass sie beide gegenwärtig im gleichen Feld zusammengehören, aufeinander einwirken. Das gestaltete Produkt ist ein konkreter Unterbau (vgl. Zinker 1982, S.61) der abstrakten Vorstellungen des Klienten. In dieser Phase müssen Therapeut und Klient mit ihm (dem Produkt) in Berührung kommen. Gefühle, die zuvor nur vage Empfindungen waren, werden jetzt erprobt und voll beleuchtet. Der Prozess kann in der Bewegung zwischen dem Gestalteten und dem Klienten anschaulich erkannt werden.

„Phenomenological theories are relational theories. In phenomenological thought, reality and perception are interactional co-constructions; they are a relationship between the perceiver and the perceived. Thus all perception and statements of reality are interpreted (Spinelli, 1989). This basic phenomenological attitude rejects the Cartesian subject-object split. There is no subjective experience that is not related to some object (intentionality); there is no experienced object except through some particular interpretive vantage point" (Yontef 2002, S. 16). 


\section{Arbeitskonzept für die Gestaltarbeit mit kreativen Medien}

Im Sinne der „interaktionalen Ko-konstruktion“ (interactional co-constructions) hat der Gestalter mit dem gestalteten Produkt die Möglichkeit zum "Gespräch“ oder zur Auseinandersetzung. Ein gestaltetes Produkt hat dann einen Eindruck, wenn es reflektiert wird, sich also in Form von einem Ausdruck zeigt. Ist es der Gestalter des Produkts, der die Resonanz durch das Produkts weitergibt, wirkt der Eindruck des Produkts wiederum auf das Produkt zurück. Damit ist ein Kreislauf etabliert. Im hier stattfindenden Prozess entwickelt sich ein Zyklus der Wechselwirkung, sozusagen: Ausdruck - Wahrnehmung - Eindruck - Resonanz - Ausdruck.

Orth und Petzold (1990) sprechen in diesem Zusammenhang vom perzeptiven (wahrnehmenden) und vom expressiven Leib und beschreiben Expression als „Formgebungsprozess nach außen (...) in die Welt und nach innen (...) in den Binnenraum der Persönlichkeit. Jedes Ausdrucksgeschehen ist zugleich auch wieder Eindruck, die beiden Ebenen sind ständig ineinander verschränkt" (S. 746ff).

Ein gestaltetes Produkt zeigt sich mehrfach mit seinen Teilen, die hinter dem Eindruck eines Produkts nicht wahrgenommen wurden. Diese Mehrdimensionalität eines Produkts muss sorgsam berücksichtigt werden. Es kann bei diesen unterschiedlichen Teilen eines Produkts mit verschiedenen Foki gearbeitet werden. Die Besonderheit eines Produkts und aller seiner Teile und die Wechselwirkung aller Teile sind wichtige Foki in der Gestaltarbeit mit dem Einsatz von kreativen Medien. Ein Fokus, an dem der Klient arbeiten will, worauf er einen besonderen Schwerpunkt setzen will, kann aufgrund eines Gefühls, von dem er besonders berührt wird, vom Klienten ausgewählt werden. Dann wird der Grund des Gefühls, das sich auf den ausgewählten Fokus bezieht, erkannt, und es kann eine weitere Motivation für die Bearbeitung unterbrochener Peozesse gewonnen werden. Somit erreicht der Klient das Kerngefühl oder den Kern des Problems, das früher nicht erkannt wurde, indem er sich um einen isolierten, zuvor unanerkannten Teil des Selbst kümmert.

In dieser Phase ist die Fragestellung „Wie und Was", wie Perls (1980) schon betont hat, weil der Schwerpunkt in der Gestalttherapie auf der phänomenologischen Beschreibung bzw. dem phänomenologischen Verstehen liegt. Es darf hier nicht um das „Warum" gehen, da "diese Frage Wahrnehmungsprozesse vorschnell in rationalisierendes, erlebnisabwehrendes Erklären bringen kann. Dadurch geht der Kontakt mit Gefühlen und Bedürfnissen verloren, und Selbsterkenntnis wird nicht als Essenz der Selbstwahrnehmung gewonnen“ (Frühmann 1994. S. 144).

Edgar und Hazley (1969) weisen im Hinblick auf chronisch Schizophrene in der poesietherapeutischen Arbeit darauf hin, wie solche Arbeit mit kreativen Medien direkten und symbolischen Ausdruck von Gefühlen ermöglicht: „Erstens bietet die 


\section{Arbeitskonzept für die Gestaltarbeit mit kreativen Medien}

Arbeit einen Ansatz zum Gespräch; zweitens kann der Klient dadurch, dass er über das Gedicht spricht, auch über sich selbst sprechen, sozusagen im ,Schutz des Gedichtes'; drittens fördern gemeinsam erlebte Gedichte die Gruppenkohäsion, was wiederum den Klienten ermutigt, etwas über sich selbst mitzuteilen. Über das gestaltete Produkt wird es dem Klienten möglich, seine Gefühle auszuleben, Katharsis zu erlangen, die Emotionen zu objektivieren und differenzieren“ (zit. nach Petzold \& Orth 1985, 38).

\subsubsection{Ausklingen lassen}

Ein gestaltete Produkt in der Therapie ist ein Objekt, das weder dem Gestalter gehört noch von ihm getrennt ist. Der Gestalter und das Produkt gehören zusammen in das Feld, in dem sie gerade sind. Das Produkt ist ein Wesen, aas in dem Prozess entsteht, ein Erfahrungsergebnis während der Gestaltung, was zur Resonanz und zur Auseinandersetzung des Gestalters führt, und eine Spur, die eigenes Land zugänglich für die Selbstexploration macht.

In dieser Phase spielt die Haltung des Therapeuten eine entscheidende Rolle für die Erlebnisqualität des Klienten. Manchmal denkt ein Klient, dass der Therapeut aufgrund des Ausgedrückten seine Seele lesen könne, dass die Arbeit mit der Gestaltung von sich schon fertig sei. Er wartet auf eine Deutung des Therapeuten, fühlt sich ganz von dem Blick des Therapeuten auf das gestaltete Produkt abhängig. Wenn sich der Therapeut das Produkt mit dem Blick wohlwollender Neugier, des Erstaunens und der Zuwendung ansieht, kann der Klient von ihm lernen, das Produkt neugierig $\mathrm{zu}$ sehen, sich selbst zu vertrauen und zu würdigen, so dass er folglich Lust hat, das Gestaltete zu explorieren. Das ist ein erster Schritt, - für weitere Schritte - zum Akzeptieren des Selbst.

Hier erlebt der Klient tiefe Verlässlichkeit von der Haltung des Therapeuten. Das Erlebnis ist insbesondere für Kinder und Jugendliche entscheidend: sich zu öffnen, ihrem Kreativitätspotential und ihrer Kreativitätslust, abseits von schlechten Erfahrungen im Unterricht, wo sie ihre Kreativität eingeschränkt erlebt haben, ohne Hindernisse begegnen zu können. Diese Erfahrung verstärkt die Motivation des Klienten zum Engagement bei der Therapie und der weiteren Arbeit, vergrößert seine Lust auf das Mitgehen in der Therapie und vertieft das Gefühl der Autonomie. Andererseits ist es wichtig, dass das Produkt vom Therapeuten nicht gedeutet und beurteilt wird, und dass der Klient ein tieferes Verständnis von sich und dem Produkt als zuvor hat. Indem der Therapeut ihn in dem Explorationsprozess unterstützt, kann 


\section{Arbeitskonzept für die Gestaltarbeit mit kreativen Medien}

der Klient die therapeutische Beziehung als einen Korrespondenzprozess verstehen.

\subsubsection{Begegnung}

Nach dem Gestalten, sozusagen dem nonverbalen Ausdruck, muss die Erfahrung, die man in dem Gestaltungsprozess erlebt hat, neu erlebt, durch verbale Arbeit integriert werden. Selbst wenn das gestaltete Produkt, z.B. Bilder oder Poesieschreiben, zur unmittelbaren und tiefen Erfahrungen geführt hat, muss es immer weiter in die verbale Arbeit hineingezogen werden, damit die Erfahrungen benannt und eingeordnet werden können.

In dieser Phase wird ein Teil von dem im Schatten liegenden Selbst (unbewusst gebliebenen Selbst) zur Kommunikation zugelassen. Zunächst begegnet der Klient sich selbst in dem gestalteten Produkt, was er früher in sich nicht gesehen hat oder was unbewusst geblieben war. Hier findet der Resonanzprozess auch zwischen Therapeut und Klient statt. Genauer gesagt hilft der Therapeut dem Klienten bei der Begegnung mit sich selbst durch Resonanz, indem er den Klienten unterstützt, an seine Fähigkeit zu glauben und sie zu entfalten, seine Selbsterforschung ausreichend tief zu erleben. Deswegen kann diese Phase ein sehr hohes Maß an emotionaler Involviertheit erzeugen.

Menschen geben Signale von sich in verschiedenen Formen, z.B. Töne, Blicke, Haltungen, Mimiken etc., mit denen sie sich zeigen und sich entwickeln. Daher gilt für die Therapie, dass Signale des Klienten seitens des Therapeuten resonieren, er sie mitschwingen lässt, so dass die Begegnung zwischen Klient und Therapeut stattfindet. Es geht hier nicht um die Förderung von Ausdruck schlechthin („Aggressionen rauslassen“), sondern um ein Verstehen des Zusammenhangs im Ausdrucksgeschehen (Weymann, 1999, S. 51), da das Ausgedrückte verstanden und sich damit auseinandergesetzt werden muss.

Die Teile des Selbst, die in dem Selbsterforschungsprozess eindeutig entdeckt werden, sind wie Mosaiksteine einer allmählich deutlicher werdenden eigenständigen Selbstwahrnehmung, sodass sie zu einer neuen Konstruktion des Selbstbildes beitragen und integriert werden. Dieser Prozess läuft immer weiter im Wachstum - das ganze Leben hindurch.

„Unsere Lebensvorgänge sind so angelegt, dass jede Zelle das Gesetz des Ganzen in sich trägt. Das bedeutet: Veränderungsprozesse vollziehen sich ganzheitlich in dem Sinn, dass der Teil immer auch eine Wirkung auf das 


\section{Arbeitskonzept für die Gestaltarbeit mit kreativen Medien}

Ganze und umgekehrt hat. Wenn Verhalten in einem Bereich verändert wird, kann diese Veränderung durchaus in anderen Bereichen wirksam werden (Thomas 1999, S. 32).

Veränderungen in der Arbeit mit dem gestalteten Produkt durch kreative Medien können stellvertretend für andere Bereiche geschehen und dazu beitragen, jene in die Gesamtgestalt des Menschen $\mathrm{zu}$ integrieren. Die Evidenzerlebnisse und Veränderungen im gestalteten Produkt mit kreativen Medien können daher zur Selbstregulierung des Menschen und zur kreativen Anpassung beitragen.

\subsubsection{Phase 4: Die Zusammenführung und Eröffnung neuer Wachstumsdimensionen}

In dieser Phase wird herauskristallisiert, dass tausende vergangener Konfigurationen die Art bestimmen, in welcher sich der Klient in Beziehung zur Welt und seinem Feld bislang selbst erschaffen und mit „unterbrochenen Prozessen oder Gefühlen“ (unfinished business) routinemäßig beschäftigt hat. Zwar nimmt der Klient wahr, was er aus der Vergangenheit als Mittel und Wege in die Gegenwart hineinbringt und ob dies im „Hier und Jetzt“ für ihn nötig ist, um mit der Situation umzugehen, und sortiert dann aus seinen Konfigurationen oder seinem Repertoire das "Unnötige" aus, was menschliches Wachstum ständig behindert hat und noch behindert. Selbstrekognition und -akzeptanz sind die Voraussetzung für Veränderung, indem der Klient in und nach dem Betrachtungs- und Reflexionsprozess seine Komplexität und Differenziertheit adäquat nutzen kann. In diesen schöpferischen, gestaltbildenden Prozessen wird die Aktivität des Ich gestützt, das Selbst zu erkennen und es in diesem Akt des Erkennens zugleich zu gestalten, wird die Leistung des Ich zum Gewinn von Identität gefördert, die nichts anderes bedeutet als „sich selbst im Lebensganzen und der Welt verstehen und begreifen zu lernen, für sich mit andren seinen Sinn, gemeinsamen Sinn zu finden“ (Petzold, 1980; Petzold \& Orth, 1985, S. 69). In diesem Prozess bewegt man sich schon zu Anfang des Wachstumsvorgangs. Eine Erfahrung der korrigierenden Beziehung mit dem Therapeuten, eine Erfahrung zur Problembewältigung, ein Erleben der eigenen Ressourcen oder des aktiven Ausdrucks ist nicht einmalig beendet, sondern wirkt weiter in anderen Lebensbereich hinein: ein sogenannter Synergieeffekt. Für Jugendliche ist ein solcher Effekt länger anhaltend als für Erwachsene (vgl. Märtens \& Petzold 1995). Sie berichten, dass die Langzeiteffekte bei der Therapie von Kindern eher größer sind als die kurzfristigen Auswirkungen. Rahm (1997) hat herausgefunden, warum die Therapieeffekte bei Kindern länger als 


\section{Arbeitskonzept für die Gestaltarbeit mit kreativen Medien}

bei Erwachsenen dauern, nämlich weil sich langfristige Auswirkung der Therapie bei Kindern möglicherweise durch wachsende Exzentrizitätsfähigkeit und damit einhergehende Identitätsentwicklung erklären lassen: Wenn sich das von einem Kind selbst entwickelte Selbstbild verändern kann, können sich Einschränkungen, Verwirrungen und Verhaltensweisen verändern. Exzentrizität kann helfen, Zuschreibungen und Botschaften von wichtigen Menschen zu relativieren (vgl. S. 107). Nach der Aktivierung dieser Exzentrizitätsfähigkeiten und nach dem aktiven Ausdruck arbeiten die Jugendlichen weiter für das neue Konstrukt ihres Wesens. Dieser Prozess führt zu einem neuen Wachstumsprozess, der sich fortwährend verändern kann. 


\section{Untersuchungsziel und Hypothesen}

\section{Untersuchungsziel und Hypothesen}

Das Ziel der vorliegenden Studie ist die Entwicklung eines indikativen Präventionsprogramms gegen Depression mit dem aktiven Einsatz kreativer Medien im Rahmen der Gestaltgruppenarbeit und die Überprüfung der Wirksamkeit des Programms. Auf Basis der vorgestellten theoretischen Untersuchungen wurde ein Programm zur Prävention von Depression konzipiert und entwickelt. Für die Anwendbarkeit der gestalttherapeutischen Behandlung von Jugendlichen wurde die Möglichkeit des Einsatzes von kreativen Medien gefunden und die Wirkpotentiale kreativer Medien zur Gestaltarbeit bei Jugendlichen untersucht. Es wurden Behandlungskonzepte, Wirkprinzipien und Phasen der Gestaltgruppenarbeit mit dem Einsatz von kreativen Medien zur indikativen Prävention von Depression bei Jugendlichen in den vorhergehenden Abschnitten im Detail herausgestellt. Das Programm soll zunächst auf seine Anwendbarkeit sowie Akzeptanz untersucht werden. Die Bestandteile des Programms bestehen aus erlebnisfördernder Exploration (als erster Schritt), aktivem Ausdruck mit der Hilfe kreativer Medien (als zweiter Schritt) und der Integration (als dritter Schritt). Teilnehmer arbeiten mit verschiedenen kreativen Medien und mit ihnen wird der Ausdruck der unterdrückten Gefühle gefördert, damit sich Selbstspaltungen und unterbrochene Prozesse oder Gefühle, auflösen können.

Die Wirkung des Programms in den verschiedenen Bereichen, in denen ich eine Veränderung erwarte, ist die zentrale Forschungsfrage. Dies geschieht im Vergleich zu einer nicht behandelten Kontrollgruppe von Jugendlichen aus der gleichen Schule, aus der die Versuchgruppe stammt. Ich erwarte von dem Präventionsprogramm nicht nur die Beseitigung oder Linderung der depressiven Symptome und der allgemeinen psychischen Belastung der Jugendlichen, sondern auch die Verbesserung in den Bereichen des psychischen Wohlbefindens.

In dieser Studie wird untersucht:

\section{Die Wirksamkeit des Programms;}

- Verändern sich vor und nach der Teilnahme an dem Programm die Symptome der Depression, die allgemeinen psychischen Beschwerden sowie das psychische Wohlbefinden?

- Verändern sie sich auch jeweils drei und sechs Monate danach? 


\section{Untersuchungsziel und Hypothesen}

\section{Der Therapieprozess;}

- Wie verläuft die Entwicklung der depressiven Symptome vom Anfang des Programms bis sechs Monate nach Ende des Programms?

- Was wird von den Teilnehmerin am Präventionsprogramm als am hilfreichsten erlebt?

Zur ersten Forschungsfrage werden folgende Hypothesen formuliert:

1. Es zeigen sich positive Veränderungen in den genannten abhängigen Variablen: depressive Symptome, psychische Beschwerden und psychisches Wohlbefinden zwischen den Ausgangswerten vor der Intervention und den Erhebungszeitpunkten nach der Intervention bei der Präventionsgruppe im Vergleich zur nicht behandelten Kontrollgruppe.

Es wird angenommen,

a) dass die Gruppe der Probanden des Präventionsprogramms nach der Intervention im Vergleich zur Kontrollgruppe signifikant größere Fortschritte bei der Reduzierung der Kernsymptome machen wird.

b) dass die Gruppe der Probanden des Präventionsprogramms nach der Intervention im Vergleich zur Kotrollgruppe bei den psychischen Bschwerden signifikant positiv verändern kann.

c) dass bei der Gruppe der Probanden des Präventionsprogramms nach der Intervention im Vergleich zur Kotrollgruppe beim psychischen Wohlbefinden eine signifikant positive Veränderung zu verzeichnen ist.

2. Es zeigen sich positive Veränderungen in den genannten abhängigen Variablen: depressive Symptome, psychische Beschwerden und psychisches Wohlbefinden drei und sechs Monate nach der Intervention bei der Präventionsgruppe im Vergleich zur nicht behandelten Kontrollgruppe.

Es wird angenommen,

a) dass die Versuchsgruppe im Vergleich zur Kontrollgruppe signifikant größere Fortschritte bei der Reduzierung der Kernsymptome im Zeitpunkt nach drei bzw. sechs Monaten nach der Intervention machen wird,

b) dass die Versuchsgruppe im Vergleich zur Kontrollgruppe signifikant positive Veränderungen bei den psychischen Beschwerden im Zeitpunkt nach drei bzw. sechs Monaten nach der Intervention erreicht, und

c) dass die Versuchsgruppe im Vergleich zur Kontrollgruppe signifikant positive 


\section{Untersuchungsziel und Hypothesen}

Veränderungen beim psychischen Wohlbefinden zum Zeitpunkt nach drei bzw. sechs Monaten nach der Intervention erreicht,

Zur Beantwortung der zweiten Frage wird Folgendes schrittweise untersucht;

- der Verlauf der depressiven Symptome von Sitzung zu Sitzung bei der Durchführung des Programms und drei bzw. sechs Monate nach Ende des Programms,

- ob es bemerkenswerte Veränderungen gibt, oder wann der Zeitpunkt der Veränderung ist, so dass die Zusammenhänge zwischen dem Zeitpunkt der Veränderung und der Inhalte der Sitzung des Programms erkannt werden können.

- welches Erlebnis in jeder Sitzung für die Teilnehmer am hilfreichsten ist, damit erstens der Prozess des Programms analysiert, zweitens das hilfreichste Erlebnis kategorisiert und folglich Wirkfaktoren des Programms herausgearbeitet werden können.

Zur Untersuchung der zweiten Frage wird die qualitative Methode zum Teil in Kombination mit der quantitativen Methode verwandt. 


\section{Das Programm GPD - J}

\section{Das Programm (GPD-J)}

\subsection{Design des Programms GPD-J}

\subsubsection{Ziel}

Das Programm heißt Gestaltgruppenarbeit zur indikativen Prävention von Depression bei Jugendlichen, abgekürzt GPD-J.

Die bisherigen Präventionsprogramme von Depression bei Jugendlichen sind eher psychoedukativ im Rahmen kognitiv-verhaltenstherapeutischer Arbeit und haben meistens das Ziel universaler Prävention von Depression. Das Programm „GPD-J“ zielt auf indikative Prävention ab, ist also für die Jugendlichen, bei denen die Symptome nicht alle klinischen Kriterien von depressiven Störungen erfüllen, sich diese Symptome aber mit hoher Wahrscheinlichkeit bei depressiven Störungen in der Zukunft ergeben könnten, konzipiert, damit die Wahrscheinlichkeit depressiver Störungen reduziert und ihre Chronifizierung verhindert wird. Wenn sich das Programm zur indikativen Prävention von Depression bei Jugendlichen als effektiv erweisen würde, könnte es zur universalen bzw. selektiven Prävention und für die Behandlung depressiver Störungen angewandt werden. Das Programm ist nicht psychoedukativ sondern psychotherapeutisch bzw. beraterisch anwendbar. Nur ein geringer Teil der Jugendlichen, die unter Depressionen leiden, ist in Behandlung. Daher ist es wichtig, den Betroffenen wenig invasive, nicht stigmatisierende und leicht erreichbare Angebote zur Verfügung zu stellen, um sie rechtzeitig vor einer Chronifizierung der Problematik zu erreichen. Hierfür ist das vorliegende Programm entwickelt.

\subsubsection{Zielgruppe und Ein- sowie Ausschlusskriterien}

Das Programm ist für 14- bis 21-jährige Jugendliche entwickelt. Das vorliegende Programm GPD-J wendet sich an Personen dieser Altergruppe mit subklinisch ausgeprägter depressiver Symptomatik. Wenn die Person mit subklinisch depressiver Symptomatik zusätzlich hohe Komorbiditätsraten, z.B. ADHD, Ess- oder Angststörungen hat, wird sie nicht in die Gruppe aufgenommen. Die Hauptzielgruppe sind 16- bis 18-jährige Jugendliche. Für eine mögliche Aufnahme in das Kursprogramm gelten folgende Einschlusskriterien: 1. Die Teilnehmer leiden an einer subklinischen 


\section{Das Programm GPD - J}

depressiven Symptomatik (Forschungsdiagnose einer leichten depressiven Episode nach DSM-IV oder Kriterien einer leichten depressiven Episode nach ICD-10 knapp verfehlt) 2. Die Teilnehmer müssen eine mindestens durchschnittliche Intelligenz mitbringen und nicht behindert sein. 3. Eine altershomogene Gruppe wird empfohlen. Als Ausschlusskriterien sind definiert: 1. das Vorliegen einer organisch bedingten psychischen Störung oder einer mentalen Retardierung, 2. akute Suizidalität oder akute stationäre Behandlungsbedürftigkeit, 3. eine Person, die gerade einen Verlust einer wichtigen Person erlebt hat, ist für die Gruppe nicht geeignet.

Eine aktive Probandenrekrutierung in Gruppen zur indizierten Prävention depressiver Symptomatik in der Schule, Jugendeinrichtungen und Beratungsstellen eröffnet die Möglichkeit, Personengruppen zu erreichen, die sich nicht selbständig bzw. nicht rechtzeitig in professionelle Hilfe begeben. Somit könnte ein wichtiger Beitrag zur Chronifizierungsprophylaxe geleistet werden.

\subsubsection{Gruppengröße}

Dieses Programm wurde so entwickelt, dass es für einen einzelnen Gruppenleiter mit einer kleinen Gruppe bis zu 12 Teilnehmern am effektivsten wäre. Ein weiterer Ko-Leiter wäre für ein angemessenes Ausmaß an Aufmerksamkeit und zur Berücksichtigung von Interessen und der Beteiligung an Gruppenaktivitäten noch effektiver. Dann könnten maximal 15 Teilnehmer an der Gruppe teilnehmen.

\subsection{Struktur des Programms GPD-J}

\subsubsection{Inhalte des Programms GPD-J}

Das Programm GPD-J umfasst 10 Arbeitssitzungen, zuzüglich einer zur Propädeutik und einer zur Rückmeldung, d.h. insgesamt 12. Jede Sitzung dauert ungefähr 120 Minuten und kann sich daher in ein Schul- oder Gemeindesetting einfügen. Jedoch kann der Zeitplan dieser Sitzungen je nach Gruppe unterschiedlich sein. Die Sitzungen sollten mindestens einmal wöchentlich stattfinden, um effektiv zu sein.

\subsubsection{Vorbereitung auf jede Sitzung}

Vor Beginn der Sitzung sollte der Gruppenleiter sicherstellen, dass genügend Stühle für alle Teilnehmer vorhanden sind. Die Stühle sollten in Kreisform aufgestellt werden, 


\section{Das Programm GPD - J}

um die aktive Teilnahme und ein Gruppengefühl zu fördern. Der Gruppenleiter sollte ebenfalls in diesem Kreis Platz nehmen. Die Sitzung sollte in einem störungsfreien bzw. angemessen großen Raum zur körperlichen Bewegung und freien Gestaltung mit kreativen Medien stattfinden. Für die Gestaltung werden Malpapier, Malutensilien, Ton, Musikgeräte usw. je nach Bedarf benötigt.

\subsubsection{Aufgabe für Teilnehmer}

Nach jeder Sitzung haben die Teilnehmer die Aufgabe, einen kleinen Text darüber zu schreiben, was sie in der jeweiligen Sitzung als am Hilfreichsten erlebt haben und den fertigen Text abzugeben. Das Ziel der Aufgabe ist zunächst das aktive Engagement und die Kontaktförderung während der Teilnahme. Am Ende des Programms wissen die Teilnehmer auf diese Weise auch, welche Sitzung sie am besten und hilfreichsten fanden. Nach der Rückmeldung bekommen sie das Tagebuch zurück.

\subsubsection{Prozessverlauf und Themen des Programms GPD-J (siehe Anhang)}

\subsubsection{Propädeutik}

Die Teilnehmer bekommen Informationen über die Prinzipien und Regeln der Gruppenarbeit. Sie stellen Fragen über das Programm oder über die Organisation und geben ihr schriftliches Einverständnis zur Teilnahme. Bei dieser Sitzung erhalten sie auch die Information über das Programms für ihre Eltern.

\subsubsection{1. Sitzung: Selbstvorstellung}

Die Teilnehmer stellen sich selbst in der Gruppe auf ihre eigene Weise vor. Somit können sie schon Kontakt zu sich selbst ermöglichen, ihre Selbstwahrnehmung verdeutlichen und ihre innere Welt zugänglich machen. In dieser Sitzung lernen sie einander kennen. Jeder Teilnehmer stellt sich zunächst mit einem Spitznamen und einem Gegenstand, den er dafür für geeignet erachtet, vor. Dabei drückt er die eigenen Wünsche an die Gruppe sowie die eigenen Bedürfnisse und Ziele aus.

\subsubsection{2. Sitzung: Familie als Lebensraum}

Die Teilnehmer malen mit dem Thema „Familie in Tieren“. Sie symbolisieren ihre 


\section{Das Programm GPD - J}

Familienmitglieder durch verschiedene Tierformen auf dem Papier mit unterschiedlichen Farben. Beim Malen auf dem Papier fordert der Therapeut jeden Teilnehmer auf, seine Aufmerksamkeit auf die Position und Richtung jedes Tieres für sich selbst und in Bezug aufeinander zu richten. Wenn beispielsweise ein Teilnehmer seinen Vater als Tiger darstellt, wie stehen dann der Tiger als Vater und z.B. ein Schaf als seine Mutter zueinander? Sehen sie einander an oder schauen sie in unterschiedliche oder sogar entgegengesetzte Richtungen? Anschließend stellen die Teilnehmer ihre Bilder in der Gruppe aus und kommunizieren darüber frei miteinander. So werden die Wahrnehmung der Lebenswelt „Familie“ und der Kontakt zum Selbst und zu den anderen gefördert. Die Teilnehmer können Probleme oder Konflikte in der Familie, aber auch Ressourcen erkennen.

\subsubsection{3. Sitzung: Schule als Lebensraum}

Nach einer Einstimmung zum Thema "Schulzeit" malen und symbolisieren die Teilnehmer ihren schulischen Entwicklungsprozess als ein Panorama auf dem Papier mithilfe von Farbstiften. Die Technik wurde vom Lebenspanorama aus der Integrativen Therapie variiert und entwickelt. Was den Teilnehmern während der Einstimmung eingefallen ist oder ihnen jetzt einfällt, drücken sie auf dem Papier aus. Nach dem Malen tauschen sie ihre Bilder miteinander aus. So bekommen sie enorme Informationen darüber, wann sie in der Schule eine schöne Zeit hatten, wann und von wem sie verletzt wurden, welche Zusammenhänge und welche Bedeutung zwischen vergangener und heutiger Zeit es für sie gibt, welche Beziehungen, welche Probleme in der Schule entstanden sind und welche Ressourcen es zur Problembewältigung gab usw.

\subsubsection{4. Sitzung: Negative Gefühle zum Selbst}

Nach einer Einstimmung malen die Teilnehmer ihr negatives Gefühl zu ihrem Selbst, also ihr Selbstbild mit negativem Gefühl. Die Foki beim Malen liegen darauf, wann sie das negative Gefühl zu sich selbst haben, wo das Gefühl vorkommt und wie sie reagieren, wenn sie das negative Gefühl zu sich selbst empfinden. Vor und beim Malen erforschen sie durch die Bilder, die in der 3. und 4. Sitzung gemalt wurden, mit wem, wann und in welchem Lebenskontext sie diese negativen Gefühle haben. Nach dem Malen benennen sie das auf dem Papier ausgedrückte Gefühl, tauschen die Bilder wieder in der Gruppe aus und geben freiwilliges Feedback oder Sharing. 


\section{Das Programm GPD - J}

\subsubsection{5. Sitzung: Ausdruck der negativen Gefühle zum Selbst}

Die Teilnehmer äußern sich freiwillig aktiv mit dem, was sie in der 4 . Sitzung gestaltet haben. Je nach "Anzeichen (Marker)", z.B. Selbstwertspaltung oder selbstunterbrochene Spaltung (siehe. Kap. 2.2) arbeiten sie mit dem Gestalteten und nutzen eventuell die Bilder, die in der 3. und 4. Sitzung gemacht wurden, damit sie anschaulich bearbeiten können, woraus das negative Gefühl stammt und worauf sich das Gefühl bezieht. Die Teilnehmer inszenieren mit dem Gestalteten ihre Polaritäten oder die unterdrückten Seiten ihres Selbst. Diese Arbeit ist als Einzelarbeit in der Gruppe angelegt. Ggf. helfen die anderen Teilnehmer dem Betroffenen aber beim Ausdruck und unterstützen ihn dabei durch Feedback (mit dem ausdrücklichen Hinweis, das Gestaltete nicht zu interpretieren), Resonanz, Sharing, etc.

\subsubsection{6. Sitzung: Unterbrochene Gefühle zu einer bestimmten Person}

Die Teilnehmer gestalten mit Ton den Ausdruck ihres Ambivalenz- oder Vermeidungsgefühls, das sich auf eine bestimmte Person bezieht. Sie holen sich die Bilder, die in der 3. und 4. Sitzung gemalt wurden, vor ihr geistiges Auge und sehen, auf wen sich das Gefühl wann bezieht. Der Therapeut fordert die Teilnehmer auf, das Gefühl nicht zu vermeiden, sondern es einfach aufs Papier zu bringen. Nach dem Malen schreiben die Teilnehmer einen kleinen Text, um das Gefühl zu benennen, und tauschen sich miteinander über ihre Tonfiguren aus.

\subsubsection{7. Sitzung: Ausdruck der unterbrochenen Gefühle zu einer bestimmten} Person

Die Teilnehmer drücken sich aktiv über das Gefühl aus, das in der 6. Sitzung mit dem Ton in eine Gestalt gebracht wurde. Hier können die Bilder, die bisher gemacht wurden, genutzt werden, damit die Teilnehmer das Gefühl gegenüber einer bestimmten Person in einer bestimmten Situation oder ihren Lebensräumen verstehen können. Sie drücken unterbrochene Prozesse oder Gefühle in Bezug auf eine bestimmte Person mit der Tonfigur aus. Diese Arbeit ist als Einzelarbeit in der Gruppe angelegt. Ggf. helfen die anderen Teilnehmer dem Betroffen beim Ausdruck und unterstützen ihn dabei (wie in der 5. Sitzung). 


\section{Das Programm GPD - J}

\subsubsection{8. Sitzung: Gestalten eigener Grenzen}

Die Teilnehmer gestalten eigene Grenzen mit Bewegung und Materialien. In dieser Sitzung haben sie noch die Chance, Restgefühle, die in der vorherigen Sitzungen noch keinen Entfaltungsraum bekommen haben, zum Ausdruck zu bringen. Sie lassen ihre Produkte, die sie bisher gestaltet haben, auf sich wirken. Danach tauschen sie sich darüber aus, was sie an neuen Erkenntnissen gewonnen haben und was sie weiter für sich tun wollen.

\subsubsection{9. Sitzung: Ich bin Ich}

Die Teilnehmer bekommen in der Einstimmung Zeit, um auf den eigenen Lebensweg zurückzuschauen und den eigenen Willen, den eigenen Lebenssinn und das eigene Lebensziel $\mathrm{zu}$ überschauen. Sie gestalten einen „Baum als Ich" mit Farben, symbolisieren ihr Selbstbild mit ihren Ressourcen, Kräften und ihrem Willen. Nach dem Gestalten tauschen sie sich miteinander über das gestaltete Produkt aus und geben einander freiwillig Feedback oder Sharing. Hier können die Teilnehmer sich mit den gestalteten Produkten, die bis dahin von dem Teilnehmer gemacht wurden, auseinandersetzen.

\subsubsection{10. Sitzung: Abschied}

Die Teilnehmer erleben sich in verschiedenen Abschiedsspielen und lernen auf diese Weise Abschied zu nehmen. Sie teilen ihre Erfahrungen in der Gruppe mit und bewerten, ob ihre Wünsche, Erwartungen oder Bedürfnisse, die sie am Anfang der Gruppe geäußert haben, erfüllt wurden.

\subsubsection{Rückmeldung}

Die Teilnehmer geben Rückmeldung über das Programm und über ihre Veränderungen während des Programms und danach. Hier werden Hilfsangebote und -momente, die für das innere Erleben der Teilnehmer effektiv zählen, analysiert. 


\section{Forschungsmethode}

\section{Forschungsmethode}

\subsection{Design des Projekts}

Das Projekt erfolgt in Form experimenteller Zwillinge, nämlich mit Versuchs- und Kontrollgruppen. In der Versuchsgruppe erleben die Teilnehmerinnen (bei der Beschreibung des Projektes bediene ich mich der weiblichen Form, da sämtliche Teilnehmer weiblich waren) das von der Forscherin entwickelte Programm zur indikativen Prävention von Depression bei 16- bis 18-jährigen Schülerinnen. Das Programm GPD-J besteht aus verschiedenen Aktivitäten mit kreativen Medien, deren Wirkpotentiale die Forscherin konzipiert hat. Die gestalttherapeutische Praxeologie und die spezifischen Behandlungskonzepte der EFT wurden für die Entwicklung des Programms konzipiert. Anschließend wurde die Methodologie der Arbeit mit kreativen Medien bei der Entwicklung des Programms unter besonderer Berücksichtigung der Behandlung Jugendlicher konzipiert. Das Projekt wurde in einer Schule für Mädchen im Alter von 16 bis 18 Jahren durchgeführt. Die Teilnehmerinnen in der Versuchsgruppe nahmen dreimal pro Woche je 2 Stunden lang an dem Programm teil, sodass die Durchführung des Programms insgesamt circa einen Monat dauerte. Neben der Versuchsgruppe wurde die Kontrollgruppe, die keine Interventionen hatte, in der Untersuchung zum Vergleich der Veränderung oben genannter Variablen (siehe. Kap. 5) - depressive Symptome, allgemeine psychische Beschwerden und psychisches Wohlbefinden - eingesetzt.

\subsection{Bedarfsanalyse}

Zunächst wurde das Oberschulamt um die Genehmigung der Durchführung der Untersuchung an Mittelschulen (entspricht 7. bis 9. Klasse in Deutschland) und Oberschulen (entspricht 10. bis 12. Klasse in Deutschland) des Oberschulamtsbezirks in der Stadt, Sokcho, Südkorea gebeten. Diese wurde im Winter 2005 erteilt. Nach der Genehmigung wurde die Information über die Untersuchung an die ausgewählten Schulen geschickt. Vier Mittelschulen, eine Berufsschule (entspricht 10. bis 12. Klasse in der Berufschule in Deutschland) und zwei Oberschulen (entspricht 10. bis 12. Klasse am Gymnasium in Deutschland) gibt es in der Stadt. Alle Schulleiter meldeten ihre Schulen zur Teilnahme an der Untersuchung an. Dann wurden die Informationsblätter über die Untersuchung durch die Beratungslehrer in jeder Schule an die Schüler 


\section{Forschungsmethode}

verteilt, damit sich die interessierten zur freiwilligen Teilnahme an der Untersuchung anmelden konnten. Da die Untersuchung nicht in einer offenen Kranken- oder Therapieinstitution für Depressionen, sondern in der Alltagssituation stattfand, war es unverzichtbar zu wissen, wie viele Schüler sich dafür interessierten. 213 Schülerinnen in vier Mittelschulen (davon sind je zwei Schulen für Mädchen bzw. für Jungen), 79 in einer Berufsschule (sie ist für Mädchen und Jungen gleichermassen), 199 in zwei Oberschulen (je eine ist für Mädchen bzw. für Jungen) waren zu der Untersuchung angemeldet. Zur Veranschaulichung folgt eine Übersicht in Tabellenform:

Tab. 7.1: Teilnahmewünsche an dem Programm

\begin{tabular}{cccc}
\hline \multirow{2}{*}{ Geschlecht } & \multicolumn{3}{c}{ Art der Schule (Anzahl) } \\
\cline { 2 - 4 } & Mittelschule (4) & Berufsschule (1) & Oberschule (2) \\
\hline männlich & 53 & 26 & 71 \\
\hline weiblich & 160 & 53 & 128 \\
\hline Gesamtzahl & 213 & 79 & 199 \\
\hline
\end{tabular}

Die Zahl der Teilnahmewünsche ist erheblich höher bei Mädchen als bei Jungen und höher in Oberschulen als in Mittelschulen. Die Durchschnittszahl der Schüler mit Teilnahmewunsch ist bei Oberschulen am höchsten, danach bei Berufschulen und schliesslich am niedrigsten bei Mittelschulen. Es erscheint merkwürdig, dass die Zahl der Schülerinnen, die eine Teilnahme an der Untersuchung wünschen, in einer Oberschule für Mädchen ziemlich groß ist, weil die gesamte Zahl der Schülerinnen, die über die Untersuchung informiert wurden, 632 war. Das bedeutet, dass fast ein Viertel der Schülerinnen vermutet, selbst unter Depressionen zu leiden, und deswegen eine therapeutische Hilfe wünscht. Die Oberschule für Mädchen wurde für die Untersuchung aufgrund hoher Motivation und eines starken Bedürfnisses der Schülerinnen ausgewählt, weil die therapeutische Arbeit besonders wirksam wird, wenn Klienten hoch motiviert sind. Vor der therapeutischen Arbeit sollte herauskristallisiert werden, wann und wie viele Schülerinnen unter depressiven Symptomen leiden, wie sie sie erleben und wie sie die Symptome zu lindern oder zu überwinden versuchen.

Als nächster Schritt zur Untersuchung dieser Fragen wurde ein Fragebogen entwickelt. Der Fragebogen beinhaltet zwei standardisierte Depressionsinventare (BDI, Depressivität von SCL-R-90, siehe Kap. 7.6.1), Fragen über soziodemographische Merkmale und offene Fragen über die Umgangs- und Bewältigungsstrategien in Alltagssituationen. Das Ziel dieses Schrittes dient nicht nur der Erhebung der 


\section{Forschungsmethode}

Antworten auf die Fragen, sondern auch der Teilnehmerauswahl für das Projekt. Die zwei Depressionsinventare werden drei Wochen danach noch einmal eingesetzt, um zu überprüfen, ob die Neigung der Schülerinnen zu Depressionen stabil geblieben ist. Depressionen bei weiblichen Jugendlichen können oft schwer eingeschätzt werden, da es kaum einen Unterschied zwischen Depressionen und depressiven Gefühlen gibt, wenn sie nur einmalig auf Depressionen untersucht werden. Die Schülerinnen, die zweimal diesen Fragebogen ausgefüllt haben, waren insgesamt 118, davon sind 38 Schülerinnen 16 Jahre, 53 Schülerinnen 17 Jahre und 27 Schülerinnen 18 Jahre alt.

\subsection{Auswahl der Teilnehmer und Gruppierung}

Die Bewerberinnen für die Teilnahme an dem Programm haben die Fragen beantwortet, die in je zwei Fragebögen BDI (21 Items) und Depressivität in SCL-R-90 (13 Items) über Depressionssymptomatik, Symptom-Check-Liste-R-90, demographische Fragen, Fragen über Beziehungen in der Familie, Gesundheit, das Leben in der Schule, Vorerfahrung in einer Psychotherapie oder einer psychologischen Beratung, das Einverständnis ihrer Eltern zur Untersuchung und für die Teilnahme ihrer Tochter sowie Erwartungen an das Programm und die Programmleiterin ausgegeben wurden. Diese Schülerinnen sollten drei Wochen nach Beantwortung des ersten Fragebogens noch einmal die Fragebögen über die Depressionssymptomatik beantworten. Der durchschnittliche BDI-Wert von 118 beantwortenden Schülerinnen war 15,932 (SD = 8,082). Nach der wiederholten Befragung über die depressive Symptomatik wurden 61 Schülerinnen, die in beiden Fragebögen zu zwei Messzeitpunkten eine hohe Punktzahl hatten, ab der höchsten Punktzahl der Reihe nach insgesamt ausgewählt, und von einem Psychiater wurde mit denjenigen Schülerinnen, bei denen Major Depression vermutet wurde, ein Interview nach DSM-IV durchgeführt. Dann wurden insgesamt 54 Schülerinnen, die subklinisch depressiv sind und in das Projekt passen (siehe Kap. 7.1), ausgewählt. Die Forscherin gab ihnen zwei Termine für die Durchführung des Programms und ließ die Schülerinnen freiwillig je nach Wunsch einen Termin davon auswählen, wodurch zwei Gruppen entstanden. In diesem Prozess konnten acht Schülerinnen keinen Termin davon auswählen, da sie wegen anderer Angelegenheiten nicht am gesamten Programm teilnehmen konnten. Die übrigen 46 Schülerinnen wurden schließlich von der Forscherin für eine zweite Gruppe, Versuchsgruppe oder Kontrollgruppe, randomisiert. Somit entstanden zwei Versuchsgruppen und zwei Kontrollgruppen. Für die Versuchsgruppe waren 23 Schülerinnen, für die Kontrollgruppe 23 Schülerinnen randomisiert. 


\subsection{Stichprobe}

Wer in der Versuchsgruppe zweimal an dem Programm nicht teilgenommen hatte und in beiden Gruppen einmal von insgesamt vier Messzeitpunkten nicht anwesend war, dessen Datei wurde in die Auswertung nicht einbezogen. Das ist bei der zweiten Versuchsgruppe und der zweiten Kontrollgruppe passiert, sodass Daten von zwei Personen nicht in die Untersuchung eingegangen sind. Eine Schülerin in der ersten Gruppe hat wegen Krankheit dreimal die Sitzungen versäumt, und eine weitere Schülerin hat in diesem Zeitraum ihre Schule gewechselt. Somit gehörten 22 Schülerinnen zu den Versuchsgruppen (in der ersten Gruppe 12, in der zweiten 10) und 22 Schülerinnen zu den Kontrollgruppen (in der ersten Gruppe 12, in der zweiten $10)$.

\subsubsection{Soziodemographische Daten}

Tab. 7.2: Soziodemographische Daten der Teilnehmerinnen

\begin{tabular}{|c|c|c|c|}
\hline Variablen & $\begin{array}{l}\text { Gesamt } \\
\mathrm{N}=44(100 \%)\end{array}$ & $\begin{array}{l}\text { Versuchsgruppe } \\
N=22(50 \%)\end{array}$ & $\begin{array}{l}\text { Kontrollgruppe } \\
\mathrm{N}=22(50 \%)\end{array}$ \\
\hline Alter & Anzahl (Prozent) & Anzahl (Prozent) & Anzahl (Prozent) \\
\hline 16 Jahre & $14(32.82)$ & 7 (15.91) & $7(15.91)$ \\
\hline 17 Jahre & $19(43.18)$ & $10(22.73)$ & $9(20.45)$ \\
\hline 18 Jahre & $11(25)$ & $5(11.36)$ & $6(13.64)$ \\
\hline Wohnsituation & Anzahl (Prozent) & Anzahl (Prozent) & Anzahl (Prozent) \\
\hline $\begin{array}{l}\text { mit Eltern } \\
\text { davon }\end{array}$ & 35 (79.55) & $17(38.64)$ & $18(40.91)$ \\
\hline $\begin{array}{l}\text { mit Stiefvater } \\
\text { davon }\end{array}$ & $1(2.27)$ & $1(2.27)$ & $0(0)$ \\
\hline mit Stiefmutter & $1(2.27)$ & $0(0)$ & $1(2.27)$ \\
\hline Wohne & & & \\
\hline $\begin{array}{l}\text { bei Mutter } \\
\text { wegen }\end{array}$ & $3(6.82)$ & $1(2.27)$ & $2(4.55)$ \\
\hline Scheidung/ Tod & $2(4.55) / 1(2.27)$ & $1(2.27) / 0(0)$ & $1(2.27) / 1(2.27)$ \\
\hline $\begin{array}{l}\text { Wohne bei Vater } \\
\text { wegen }\end{array}$ & $5(11.36)$ & $3(6.82)$ & $2(4.55)$ \\
\hline Scheidung/Tod & $4(9.09) / 1(2.27)$ & $2(4.55) / 1(2.27)$ & $2(4.55) / 0(0)$ \\
\hline Sonstiges & $1(2.27)$ & $1(2.27)$ & $0(0)$ \\
\hline
\end{tabular}




\begin{tabular}{llll}
\hline Geschwister & Anzahl (Prozent) & Anzahl (Prozent) & Anzahl (Prozent) \\
Bin Einzelkind & $12(27.27)$ & $5(11.36)$ & $7(15.91)$ \\
Habe ältere & $10(22.73)$ & $6(13.64)$ & $4(9.09)$ \\
$\begin{array}{l}\text { Schwestern } \\
\text { Habe jüngere }\end{array}$ & $7(15.91)$ & $3(6.82)$ & $4(9.09)$ \\
Schwestern & & & \\
Habe ältere Brüder & $9(20.45)$ & $4(9.09)$ & $5(11.36)$ \\
Habe jüngere Brüder & $9(20.45)$ & $5(11.36)$ & $4(9.09)$ \\
\hline
\end{tabular}

Die Anzahl der Geschwister ist meistens zwei. Drei Schülerinnen haben drei Geschwister (Tab. 8.2).

Sonstige Fragen betreffen den Beruf der Eltern und Geschwister. Als Beruf des Vaters wurde Unternehmer, Angestellter, Bankbeamter, Polizist, Professor, Architekt, Taxifahrer, Bibliothekar, Skripter, Pfarrer usw., aber keiner als Arbeitsloser angegeben. Als Beruf der Mutter wurde Hausfrau, Ernährungsberaterin, Lehrerin, Unternehmerin, Angestellte, Floristin, Friseurin und Beamtin genannt. Am häufigsten wurde der Beruf des Vaters als Unternehmer (27\%) und der der Mutter als Hausfrau (29\%) angegeben. Die Berufe von Geschwistern waren zu 100\% Schüler oder Studenten.

\subsubsection{Gesundheit und Konsumverhalten}

\subsubsection{Subjektive Einschätzung zur Gesundheit}

Die Teilnehmerinnen schätzten ihre Gesundheit subjektiv so ein, dass sie „keine gesundheitlichen Schwierigkeiten" bei sich fanden. Trotzdem berichteten fast alle Teilnehmerinnen über chronische Kopfschmerzen, Konzentrationsschwierigkeiten und Bauchschmerzen. Die Teilnehmerinnen hatten innerhalb der letzten fünf Jahre keine Unfälle und keine Operationen wegen gesundheitlicher Probleme.

\subsubsection{Konsumverhalten}

Unter dem Thema werden der Konsum von Alkohol und Zigaretten abgefragt. Die Antworten zum Thema Alkohol lauteten "trinke keinen Alkohol“ (N=35, 79.55\%), „1 bis $3 \mathrm{Mal}$ in einem Semester" (N=4, 9.09\%), "1 bis $3 \mathrm{Mal}$ in einem Monat" (N=3, 6.81\%), „1 Mal pro Woche“ (N=2, 4.55\%). Die Antworten zum Thema Rauchen lauteten "rauche nicht" (N=40, 90.91\%), „1 bis $3 \mathrm{Mal}$ in einem Semester" (N=2, 4.55\%), "1 bis 3 Mal in einem Monat" $(\mathrm{N}=1,2.27 \%)$, „1 bis 3 Mal pro Woche“ (N=1, 2.27\%). Die zwei 


\section{Forschungsmethode}

Schülerinnen, die mindestens 1 bis $3 \mathrm{Mal}$ in einem Monat rauchten, konsumierten auch häufig Alkohol. Aber die drei Schülerinnen, die mindestens 1 bis $3 \mathrm{Mal}$ in einem Monat tranken, verhielten sich unabhängig davon beim Rauchen. Die regelmäßige Einnahme eines Medikamentes kam bei niemandem vor.

\subsubsection{Beziehung zur Familie}

Dieser Frage wurde in 10-Prozent-Skalen (10\% bis 100\%) nachgegangen. Die Frage lautete, wie tief die Schüler mit jedem der eigenen Familienmitglieder vertraut sind. Die Schülerinnen berichteten, dass die Beziehung zu den Eltern eindeutig enger zur Mutter ( $\mathrm{N}=39, \mathrm{M}=65.13, \mathrm{SD}=16.68)$ als zum Vater $(\mathrm{N}=41, \mathrm{M}=50.24, \mathrm{SD}=16.65)$ ist. Die folgende Tabelle 7.3 zeigt die Befunde über die Beziehung zu den Eltern. Die Schülerinnen, die mit ihrem Vater oder ihrer Mutter wegen Scheidung oder Tod nicht zusammenlebten, antworteten auf diese Fragen nicht.

Tab. 7.3: Beziehung zu ihren Eltern der Teilnehmerinnen

\begin{tabular}{lcc}
\hline Beziehungstiefe $(\%)$ & Mit Vater( Anzahl / \%) N=41 & Mit Mutter (Anzahl / \%) N=39 \\
\hline 10 (fast gar nicht) & 12.4 & $0 / 0$ \\
20 & $2 / 4.9$ & $1 / 2.6$ \\
30 & $5 / 12.2$ & $2 / 5.1$ \\
40 & $7 / 17.1$ & $1 / 2.6$ \\
50 & $8 / 19.5$ & $5 / 12.8$ \\
60 & $7 / 17.1$ & $8 / 20.5$ \\
70 & $9 / 22.0$ & $10 / 25.6$ \\
80 & $1 / 2.4$ & $9 / 23.1$ \\
90 & $0 / 0$ & $3 / 7.7$ \\
100 (sehr tief) & $0 / 0$ & $0 / 0$ \\
\hline
\end{tabular}

\subsubsection{Stressor, körperliches Befinden und Bewältigungsstrategie}

Die Schülerinnen beantworteten zu dem Thema drei offene Fragen: 1. was für sie der stärkste Stressfaktor ist, 2. welcher Körperteil sich unangenehm anfühlt, wenn sie Stress haben. und 3. was sie am häufigsten machen, um den Stress zu überwinden.

Die meisten Schülerinnen nannten als den stärksten Stressfaktor den Lerndruck $(88.64 \%)$, wie es in Tabelle 7.4. zu sehen ist.

Der Kopf machte einen großen Anteil (34.09\%) der unangenehmen Körperteile bei 


\section{Forschungsmethode}

Stress aus. Dann kamen in der Rangfolge der Bauch (22.73\%), der Magen (15.9\%), das Herz (11.36\%), die Brust (6.82\%), die Augen (4.55\%) und die Schultern (4.55\%).

Die Schülerinnen versuchten mit Schlafen (20.45\%), SMS schreiben und ihren Freunden schicken (13.64\%), Essen (11.36\%), Schreiben (9.09\%) und Musik hören (9.09\%), ihren Stress zu überwinden. Die Bewältigungsstrategien fanden zur Hälfte allein (52.27\%) statt (siehe Tab. 7.5).

Tab. 7.4: Der stärkste Stressfaktor der Teilnehmerinnen (N=44)

\begin{tabular}{ll}
\hline $\begin{array}{l}\text { Lerndruck } \\
(39 / 88.64 \%)(\text { Anzahl/\%) }\end{array}$ & $\begin{array}{l}\text { Beziehungskonflikt } \\
(5 / 11.36 \%)(\text { Anzahl/\%) }\end{array}$ \\
\hline Note $(15 / 34.09)$ & Mit Mutter $(2 / 4.55)$ \\
Prüfung $(9 / 20.45)$ & Mit Freunden $(3 / 6.82)$ \\
Widerrede von Eltern (8/18.18) & \\
Übermäßige Anforderung (4/9.09) & \\
Übermäßige Erwartung von Eltern (3/6.82) & \\
\hline
\end{tabular}

Tab. 7.5: Stressbewältigungsstrategien von Teilnehmerinnen ( $\mathrm{N}=44)$

\begin{tabular}{lll}
\hline Allein (23/52.27\%) & Mit Medien (12/27.27\%) & Interpersonal (3/6.82\%) \\
\hline Schlafen (9/20.45) & Musik hören (4/9.09) & Freunde treffen (2/4.55) \\
Essen (5/11.36) & Internetsurfen (3/6.82) & Sprechen mit Mutter (1/2.27) \\
Schreiben (4/9.09) & Fernsehen (2/4.55) & \\
Schreien (3/6.82) & Film gucken (2/4.55) \\
Laut weinen (1/2.27) & Sport treiben (1/2.27) \\
\cline { 2 - 3 } Lernen (1/2.27) & SMS schreiben u. schicken zu Freunden (6/13.64) \\
\hline
\end{tabular}

\subsubsection{Das Leben in der Schule}

$\mathrm{Zu}$ diesem Thema wurden vier Fragen gestellt: Grundstimmung in der Schule, allgemeine Zufriedenheit mit dem Leben in der Schule, Zufriedenheit in der Beziehung zu Schulkameraden bzw. zu Lehrern. Die Frage über die Grundstimmung in der Schule beantworteten die Schülerinnen in freier Formulierung. Die Antworten auf die Frage nach der Grundstimmung wurden jeweils durch ein Adjektiv formuliert. Bei den anderen drei Fragen wurde nach 10-Prozent-Skalen (10\% bis 100\%) vorgegangen. 


\section{Forschungsmethode}

\subsubsection{Grundstimmung in der Schule}

Die Schülerinnen benannten ihre Grundstimmung in der Schule mit den unten stehenden Adjektiven, die in der folgenden Tabelle 7.6 sortiert sind. Über zwei Drittel berichten negativ ihre Grundstimmung in der Schule.

Tab. 7.6: Grundstimmung der Teilnehmerinnen in der Schule

\begin{tabular}{lll}
\hline Positiv (Anzahl/Prozent) & Neutral (Anzahl/Prozent) & Negativ (Anzahl/Prozent) \\
Gesamt (8/18.18) & Gesamt (4/9.09) & Gesamt (32/72.73) \\
\hline Gut (2/4.55) & Keine Stimmung (3/6.82) & Müde $(9 / 20.45)$ \\
Relativ gut (3/6.82) & Wechselhaft (1/2.27) & Deprimiert (6/13.64) \\
Froh (3/6.82) & & Langweilig $(7 / 15.91)$ \\
& & Bedrückt (3/6.82) \\
& Gereizt $(2 / 4.55)$ \\
& Traurig $(3 / 6.82)$ \\
& & Atemlos $(2 / 4.55)$ \\
\hline
\end{tabular}

\subsubsection{Allgemeine Zufriedenheit mit dem Leben in der Schule}

Die Frage war, wie sehr die Teilnehmerinnen allgemein mit dem Umgang in der Schule zufrieden sind. Die Schülerinnen konnten die Frage in 10-Prozent-Skalen beantworten. Die durchschnittliche Zufriedenheit mit dem Leben in der Schule lag bei $45.90 \%$ (SD 18.71).

\subsubsection{Zufriedenheit in der Beziehung zu Schulkameraden bzw. Lehrern}

Die beiden Fragen lauteten, wie sehr die Teilnehmerinnen zufrieden in der Beziehung zu Schulkameraden bzw. Lehrern sind. Die Zufriedenheit in der Beziehung zu Schulkameraden lag höher (M=64.77, SD=17.71) als mit Lehrern (M=56.36, SD=19.42).

\subsubsection{Vorerfahrung in der Teilnahme an einer Psychotherapie oder einer psychologischen Beratung}

Die Teilnehmerinnen hatten keine Vorerfahrung in der Teilnahme an einer Psychotherapie oder an einer psychologischen Beratung. 


\section{Forschungsmethode}

\subsection{Durchführung des Programms GPD-J}

Das Programm wurde für die erste Versuchsgruppe im Zeitraum vom 23. Juli bis 18. August 2006, für die zweite Versuchsgruppe vom 13. August bis 8. September 2006 in ihrer Schule durchgeführt. Es bot für die Teilnehmerinnen dreimal pro Woche nach dem Unterricht jeweils 120 Minuten an, inklusive einer jeweils 10-minütige Pause. Der Arbeitsraum war über $100 \mathrm{~m}^{2}$ groß, und eine Stereoanlage, sechs Arbeitstische für gestalterisches Arbeiten und zahlreiche bequeme Stühle standen zur Verfügung.

Das Programm wurde von der Autorin durchgeführt. Sie nimmt seit 1999 an einer Ausbildung zur Gestaltherapeutin innerhalb einer Weiterbildungsgruppe teil, studierte Counselling Psychologie bzw. Koreanistik und Pädagogik, arbeitete elf Jahre als Lehrerin bzw. Beratungslehrerin an einer Oberschule in Korea (entspricht der 10. bis 12. Klasse am Gymnasium in Deutschland). Für die therapeutische Anwendung kreativer Medien nahm sie zwei Jahre lang an einer Weiterbildung für Integrative Gestalttherapie am Fritz Perls-Institut teil.

\subsection{Erhebungsinstrument}

Hier werden die Instrumente zur Erfassung des Therapie- oder psychischen Beratungserfolgs dargestellt, welche $\mathrm{zu}$ allen diagnostischen und katamnestischen Untersuchungen eingesetzt werden. Hier wird entsprechend der Kategorisierung von Schulte (1993) zwischen Symptomfragebögen und Massstäben für die Folgen der Störung zur quantitativen Untersuchung unterschieden und angemessene Instrumente ausgewählt und eingesetzt. Das Ergebnis einer Therapie oder Beratung kann durch Veränderungsmessung bestimmt werden. Dabei werden Messwerte (Statusmaße) zu verschiedenen Zeitpunkten errechnet, beispielsweise in Form einer Prä-PostEffektstärke (Grawe \& Braun, 1994). Hierzu können einerseits störungsübergreifende Instrumente genutzt werden, andererseits können aber auch störungsspezifische Verfahren entsprechend der jeweiligen ICD-10 bzw. DSM-IV-Diagnosen des Klienten zum Einsatz kommen (Vocks, et al., 2005). In vorliegender Arbeit werden Symptomfragebögen als störungspezifisches Maß vorgelegt: das Beck's Depressions Inventory (BDI; Beck, 1978) und Symptom-Check-Liste (SCL-90-R; Derogatis, 1986). Unter die Kategorie „Folge der Störung“ werden Fragebögen subsumiert, welche die Beeinträchtigung erfassen, die der Klient durch seine Störung in verschiedenen Lebens- und Funktionsbereichen erlebt. In diesem Zusammenhang ist allerdings anzumerken, dass die erfassten Konstrukte nicht in jedem Falle ausschließlich den 


\section{Forschungsmethode}

Folgen einer Störung zuzuordnen sind, sondern möglicherweise auch bei der Entstehung psychischer Störungen von Relevanz sein können (Vocks, et al. 2005, S. 100). Zur Erfassung der Beeinträchtigung, die der Klient in verschiedenen Lebensbereichen erlebt, wird Psychological Well-Being Scale (PWB, Ryff 1989) eingesetzt, wobei Lebensqualität und Funktionsfähigkeit erfragt werden. Neben den oben genannten Verfahren, die primär auf die Erfassung des Therapie-Outcomes fokussieren, beantworten die Teilnehmerinnen Fragen zum Therapieprozess, welche nach jeder Sitzung vorgelegt werden und qualitativ untersucht werden. Dies beschreibe ich im Kap. 7.6.2.

\subsubsection{Zur Erhebung der Wirksamkeit des Programms GPD-J}

\subsubsection{Zur Erfassung depressiver Symptombelastung}

\subsection{BDI (Beck's Depression Inventory)}

Das Beck's Depression Inventory ist ein international anerkanntes Selbstbeurteilungsinstrument zur Erfassung der depressiven Symptombelastung. In der Untersuchung wird die von Lee und Song (1991) übersetzte koreanische standardisierte Version des Beck's Inventory (1978) eingesetzt. Der Fragebogen fragt depressive Symptome ab, die mit einer vierstufigen Skala beantwortet werden können. Beim BDI handelt es sich um ein 21 Items umfassendes Instrument, das nach Häufigkeit (Ränge von 0-3) von depressiven Symptomen in den vergangenen sieben Tagen fragt. Diese werden beim Berechnen des Gesamtscores umgepolt und schließlich mit den anderen Items $\mathrm{zu}$ einem Summenscore zusammengefasst. Aus der Summierung der zwischen 0 und 3 kodierten Items ergibt sich ein maximaler Score von 63 und ein minimaler Wert von 0. Die interne Konsistenz betrug in dieser Untersuchung Cronbach's Alpha $=.959$.

Aus Lee und Songs Studie (1991) ergab sich, dass man bei Werten zwischen 16 und 23 von subliminaler Depression spricht, während Werte ab 24 auf eine klinisch manifeste Depression hinweisen.

\subsection{Depressivität in der SCL-90-R}

Zur Erfassung der Depressivität wird die Symptomcheckliste-90-R über „Depressivität“ mit 13 Items der koreanischen Version verwandt. Der umfassende 


\section{Forschungsmethode}

Fragebogen erfasst die aktuelle Belastung von Probanden während der letzten sieben Tage. Die Antwortmöglichkeiten werden mit 0 (überhaupt nicht) bis 4 (sehr stark) kodiert, summiert und der Mittelwert errechnet. Aus der Summierung ergeben sich ein maximaler Score von 52 und ein minimaler Wert von 0.

\subsubsection{Zur Erfassung allgemeiner psychischer Beschwerden: Die Symptomcheckliste (SCL-90 Revised)}

Die Symptom Checkliste (SCL-90-R, Derogatis et al. 1977) ist ein störungsübergreifendes Selbstbeurteilungsinstrument zur Erfassung subjektiver Beeinträchtigungen durch multidimensionale psychische Symptombelastungen. In der Untersuchung wird die auf koreanisch standardisierte Symptom-Checkliste (Kim et al., 1984) benutzt. Die SCL-90-R ist bei Jugendlichen ab 14 Jahren und bei Erwachsenen anwendbar, vor allem im ambulanten Setting, und verfügt über gute psychometrische Kennwerte. Es handelt sich um das international am häufigsten verwendete störungsübergreifende Maß zur Bestimmung der Wirksamkeit von Psychotherapie (vgl. Geiser et al. 2000; Geisheim et al. 2002; Ogles, Lambert \& Masters 1996; Strupp, Horowitz \& Lambert 1997). Der umfassende Fragebogen erfasst die aktuelle Belastung von Probanden durch 90 psychische und körperliche Symptome mit zeitlichem Bezug auf die letzten sieben Tage. Die fünf Ausprägungsstufen lauten: „überhaupt nicht“ (0) „ein wenig“ (1) „ziemlich“ (2) „stark“ (3) „sehr stark“ (4); die Beantwortungszeit beträgt 10 bis 15 Minuten. Die Antwortmöglichkeiten werden mit 0 (überhaupt nicht) bis 4 (sehr stark) kodiert, summiert und der Mittelwert berechnet.

In der koreanischen Version verteilen sich die 90 Items auf 10 Skalen, die sich in der englischen Version auf 9 Skalen verteilen. Die 10 Skalen beschreiben folgende Symptomdimensionen und die drei globalen Kennwerte (siehe Tabelle 7.7). GSI mit PSDI und PTS wird zur Bewertung des klinischen Bildes von Patienten eingesetzt (Derogatis et al. 1977, vgl. Kim et al. 1984). 


\section{Forschungsmethode}

Tab. 7.7: Symptom-Checkliste 90-R Skalen und globale Kennwerte in der koreanischen Version

\begin{tabular}{|c|c|c|}
\hline Skala & $\begin{array}{l}\text { Bezeichnung } \\
\text { (Items/Fragenanzahl) }\end{array}$ & Inhalt \\
\hline SOMA & Somatisierung (12) & $\begin{array}{l}\text { einfache körperliche Belastung bis zu funktionellen } \\
\text { Störungen }\end{array}$ \\
\hline ZWAN & Zwanghaftigkeit (10) & $\begin{array}{l}\text { leichte Konzentrationsunfähigkeiten bis zu } \\
\text { ausgeprägter Zwanghaftigkeit }\end{array}$ \\
\hline UNSI & $\begin{array}{l}\text { Unsicherheit im } \\
\text { Sozialkontakt (9) }\end{array}$ & $\begin{array}{l}\text { leichte soziale Unsicherheit bis zum Gefühl völliger } \\
\text { persönlicher Minderwertigkeit }\end{array}$ \\
\hline DEPR & Depressivität (13) & Traurigkeit bis hin zur schweren Depression \\
\hline ÄNGS & Ängstlichkeit (10) & körperlich spürbare Nervosität bis zu tiefer Angst \\
\hline AGGR & $\begin{array}{l}\text { Aggressivität / } \\
\text { Feindseligkeit (6) }\end{array}$ & $\begin{array}{l}\text { Reizbarkeit und Aggressivität bis zu starker } \\
\text { Feindseligkeit }\end{array}$ \\
\hline PHOB & Phobische Angst (7) & $\begin{array}{l}\text { leichtes Gefühl von Bedrohung durch bestimmte } \\
\text { Gegenstände bis zur massiven phobischen Angst }\end{array}$ \\
\hline PARA & Paranoides Denken (6) & $\begin{array}{l}\text { Misstrauen, projizierendes Denken bis zu starkem } \\
\text { paranoiden Denken }\end{array}$ \\
\hline PSYC & Psychotizismus (10) & $\begin{array}{l}\text { mildes Gefühl der Isolation und Entfremdung bis zur } \\
\text { dramatischen Evidenz der Psychose }\end{array}$ \\
\hline ZUSÄ & Zusätzliche Items (7) & $\begin{array}{l}\text { Die Items, die nicht in die Skalen } 1 \text { bis } 9 \text { gehören oder } \\
\text { ausgewertet werden, aber klinisch wichtige } \\
\text { Bedeutung haben. Die Items werden in Bezug auf die } \\
\text { gesamte Konfiguration benutzt ohne die Deutung der } \\
\text { Anzahlswerte }\end{array}$ \\
\hline \multicolumn{3}{|c|}{ Globale Kennwerte } \\
\hline GSI & Global Severity Index & $\begin{array}{l}\text { misst die grundsätzliche psychische Belastung } \\
\text { (errechnet aus der Summe der Summenwerte der } \\
\text { Skalen (Grand Total) dividiert durch die Anzahl der }\end{array}$ \\
\hline PST & Positive Symptome & Items $(90))$ \\
\hline & Total & Anzahl der Symptome (errechnet als Summe der \\
\hline PSDI & $\begin{array}{l}\text { Positive Symptome } \\
\text { Distress Index }\end{array}$ & $\begin{array}{l}\text { Belastungstendenzen je Skala, Anzahl der Items }>0 \text { ) } \\
\text { misst die Intensität der Antworten (errechnet als } \\
\text { Quotient der Gesamtsumme (Grand Total) und dem } \\
\text { PST), zeigt sich als Stil der Antworten des Patienten }\end{array}$ \\
\hline
\end{tabular}




\section{Forschungsmethode}

Die teststatistischen Kennwerte des SCL-90-R in der koreanischen Version (Kim et al., 1984) gründen sich auf einer Normalpopulation $(\mathrm{N}=4205)$, davon erhoben an Jugendgruppen im Alter 16 bis 18 (N=1907). Für die Normierung von Jugendproben im koreanischen Sprachraum wurden 894 männliche und 1013 weibliche Probanden im Alter von 16 bis 18 Jahren von Kim und seinen Kollegen herangezogen (Tab. 7.8).

Tab. 7.8: Mittelwert und Standardabweichung der SCL-90-R in der Jugendstichprobe der Normalbevölkerung (Kim et al., 1984)

\begin{tabular}{lcccc}
\hline \multirow{2}{*}{ Bezeichnung } & \multicolumn{2}{c}{ Männliche (N=894) } & \multicolumn{2}{c}{ Weibliche (N=1013) } \\
\cline { 2 - 5 } & $\mathrm{M}$ & $\mathrm{SD}$ & $\mathrm{M}$ & $\mathrm{SD}$ \\
\hline Somatisierung & 0.65 & 0.55 & 0.77 & 0.59 \\
Zwanghaftigkeit & 1.34 & 0.64 & 1.26 & 0.59 \\
Interpersonale & 1.22 & 0.63 & 1.23 & 0.61 \\
Unsicherheit & & & & \\
Depressivität & 1.04 & 0.66 & 1.08 & 0.67 \\
Ängstlichkeit & 0.90 & 0.68 & 0.93 & 0.66 \\
Feindseligkeit & 1.03 & 0.78 & 0.92 & 0.69 \\
Phobische Angst & 0.52 & 0.51 & 0.56 & 0.53 \\
Paranoides Denken & 0.87 & 0.62 & 0.77 & 0.57 \\
Psychotizismus & 0.77 & 0.62 & 0.68 & 0.58 \\
\hline GSI & 0.93 & 0.53 & 0.93 & 0.52 \\
PSDI & 1.61 & 0.44 & 1.53 & 0.43 \\
PST & 49.67 & 19.19 & 51.73 & 17.98 \\
\hline
\end{tabular}

7.6.1.3 Zur Erfassung psychischen Wohlbefindens: Psychological Well-Being Scale (PWBS)

Das Inventar "Psychological Well-Being Scale" (PWB-S) wurde als ein Selbstbeurteilungsinstrument von Ryff (1989b) entwickelt. Der 46 Items umfassende Fragebogen erfasst die Lebensqualität, die Ryff "psychisches Wohlbefinden" (psychological Well-Being) nannte. Ryff (1989c, vgl. S. 1070) entwickelte den Fragebogen zur Erfassung individueller Lebensqualität auf Grund psychologischer Theorien, z.B. Perspektiven oder Konzepte von Maslows „selfactualization" (1968), Rogers' "fully functioning person“ (1961), Jungs „individuation“ (1933), Allports „maturity“ (1961) und „life span developmental“ Perspektive von Eriksons „psychological stage model“ (1959), Buhlers 


\section{Forschungsmethode}

„basic life tendencies" (1935) und Neugartens „personality change“ (1968, 1973). Das psychische Wohlbefinden (psychological Well-Being) ist die Summe der psychischen Aspekte individueller Lebensqualität und wird vom subjektiven Zufriedenheitsgefühl und dem positiven oder negativen Gefühl unterschieden. Ryff (1989a, b, c; Ryff \& Keyes 1995; Schumutte \& Ryff, 1997) betonte, dass sich die Lebensqualität nicht nur auf subjektive Zufriedenheit oder Glücklichsein bezieht, sondern dass man als Mitglied in der Gesellschaft gut integriert ist, wenn man eine hohe Lebensqualität hat.

Die 46 Items des Fragebogens erfragen sechs Dimensionen, die aus "self acceptance" (SA), "positive relations with others" (PR), "autonomy“ (AU), „environmental mastery“ (EM), „purpose in life“ (PL) und „personal growth“ (PG) bestehen. In der Summe weist die Integration der Geistesgesundheit, der klinischen und „Life-Span“ Entwicklungstheorien auf mehrfache konvergierende Aspekte der positiven psychischen Funktion hin. Die Dimensionen wurden von mir ins Deutsche übersetzt (siehe Tab. 7.9).

Tab. 7.9: Definitionen der Dimensionen des psychischen Wohlbefindens (Ryff, 1989)

\begin{tabular}{|c|c|}
\hline \multicolumn{2}{|c|}{ Dimension 1. Selbstakzeptanz (8 Items) } \\
\hline $\begin{array}{l}\text { Proband mit hoher } \\
\text { Punktzahl }\end{array}$ & $\begin{array}{l}\text { hat eine positive Haltung zum Selbst; erkennt und akzeptiert viele } \\
\text { unterschiedliche Aspekte von sich selbst, einschließlich der guten und } \\
\text { schlechten Qualitäten; hat eine positive Einstellung gegenüber der } \\
\text { eigenen Vergangenheit. }\end{array}$ \\
\hline $\begin{array}{l}\text { Proband mit } \\
\text { niedriger Punktzahl }\end{array}$ & $\begin{array}{l}\text { ist unzufrieden mit sich selbst; ist enttäuscht darüber, was ihm/ihr in } \\
\text { der Vergangenheit passiert ist; hat Probleme mit bestimmten } \\
\text { Eigenschaften von sich selbst; möchte anders sein, als er/sie ist. }\end{array}$ \\
\hline \multicolumn{2}{|c|}{ Dimension 2. Positive Beziehungen zu anderen (7 Items) } \\
\hline $\begin{array}{l}\text { Proband mit hoher } \\
\text { Punktzahl }\end{array}$ & $\begin{array}{l}\text { hat warme, erfüllende, vertrauensvolle Beziehungen zu anderen; ist } \\
\text { bemüht um das Wohlbefinden anderer; fähig zu starker Empathie, } \\
\text { Zuneigung und Intimität; er/sie weiß um das Geben und Nehmen in } \\
\text { menschlichen Beziehungen. }\end{array}$ \\
\hline $\begin{array}{l}\text { Proband mit } \\
\text { niedriger Punktzahl }\end{array}$ & $\begin{array}{l}\text { hat wenig enge und vertrauensvolle Beziehungen } \mathrm{zu} \text { anderen; hat } \\
\text { Schwierigkeiten, warm, offen und um andere besorgt } \mathrm{zu} \text { sein; ist } \\
\text { hinsichtlich zwischenmenschlicher Beziehungen isoliert und frustriert; } \\
\text { ist nicht bereit, Kompromisse einzugehen, um wichtige Beziehungen } \\
\text { mit anderen zu erhalten. }\end{array}$ \\
\hline
\end{tabular}




\section{Forschungsmethode}

\begin{tabular}{|c|c|}
\hline \multicolumn{2}{|c|}{ Dimension 3. Autonomie/Selbstbestimmung (8 Items) } \\
\hline Proband mit hoher & ist selbstbestimmt und unabhängig; ist fähig, in gesellschaftlichen \\
\hline Punktzahl & $\begin{array}{l}\text { Zwängen auf bestimmte Arten } \mathrm{zu} \text { denken, zu handeln und } \mathrm{zu} \\
\text { widerstehen; reguliert das eigene Verhalten von innen; bewertet sich } \\
\text { selbst anhand persönlicher Standards. }\end{array}$ \\
\hline $\begin{array}{l}\text { Proband mit } \\
\text { niedriger Punktzahl }\end{array}$ & $\begin{array}{l}\text { ist abhängig von den Erwartungen und Bewertungen anderer; ist bei } \\
\text { wichtigen Entscheidungen auf die Urteile anderer angewiesen; passt } \\
\text { sich sozialen Zwängen auf bestimmte Arten zu denken und zu } \\
\text { handeln an. }\end{array}$ \\
\hline \multicolumn{2}{|c|}{ Dimension 4. Beherrschung der Lebenswelt (8 Items) } \\
\hline Proband mit hoher & ist souverän und kompetent in der Gestaltung der Lebenswelt; hat die \\
\hline Punktzahl & $\begin{array}{l}\text { Kontrolle über eine komplexe Lebenswelt und vielfältige äußerliche } \\
\text { Aktivitäten; bedient sich effektiv gegebener Ressourcen; ist fähig, } \\
\text { Bedingungen herzustellen bzw. auszuwählen, die den eigenen } \\
\text { Bedürfnissen und Werten entsprechen. }\end{array}$ \\
\hline $\begin{array}{l}\text { Proband mit } \\
\text { niedriger Punktzahl }\end{array}$ & $\begin{array}{l}\text { hat Schwierigkeiten, den Alltag zu organisieren; fühlt sich unfähig, die } \\
\text { eigene Umgebung zu ändern bzw. zu verbessern; ist sich gegebener } \\
\text { Ressourcen nicht bewusst; hat keinen Organisationssinn für äußerliche } \\
\text { Gegebenheiten. }\end{array}$ \\
\hline \multicolumn{2}{|c|}{ Dimension 5. Lebenssinn (7 Items) } \\
\hline $\begin{array}{l}\text { Proband mit hoher } \\
\text { Punktzahl }\end{array}$ & $\begin{array}{l}\text { hat Ziele für das eigene Leben und ist zielstrebig; hat das Gefühl, dass } \\
\text { das gegenwärtige und vergangene Leben sinnvoll ist; hat Grundsätze, } \\
\text { die dem eigenen Leben einen Sinn geben. }\end{array}$ \\
\hline $\begin{array}{l}\text { Proband mit } \\
\text { niedriger Punktzahl }\end{array}$ & $\begin{array}{l}\text { hat kein Gespür für einen Sinn des eigenen Lebens; hat wenig Ziele } \\
\text { und ist orientierungslos; erachtet das vergangene Leben als } \\
\text { bedeutungslos; hat keine Perspektive oder Grundsätze, die dem } \\
\text { Leben einen Sinn verleihen. }\end{array}$ \\
\hline \multicolumn{2}{|c|}{ Dimension 6. Persönliches Wachstum (8 Items) } \\
\hline $\begin{array}{l}\text { Proband } \\
\text { mit } \\
\text { hoher Punktzahl }\end{array}$ & $\begin{array}{l}\text { hat ein Empfinden anhaltender Selbstentwicklung; erachtet sich selbst } \\
\text { als im Wachstum und in Selbstverwirklichung begriffen; ist neuen } \\
\text { Erfahrungen gegenüber aufgeschlossen; hat ein Gespür für die } \\
\text { Verwirklichung des eigenen Potentials; sieht sich selbst und das } \\
\text { eigene Verhalten mit der Zeit verbessert; verändert sich in einer Art } \\
\text { und Weise, in der sich Selbstkenntnis und Effektivität widerspiegeln. }\end{array}$ \\
\hline
\end{tabular}




\section{Forschungsmethode}

Proband mit

niedriger Punktzahl hat ein Empfinden persönlicher Stagnation; hat nicht das Gefühl, sich selbst mit der Zeit zu verbessern und zu vervollkommnen; ist vom Leben gelangweilt und nicht am Leben interessiert; fühlt sich unfähig, neue Sicht- und Verhaltensweisen zu entwickeln.

Die Antwortmöglichkeiten werden mit 1 (ganz wenig oder gar nicht) bis 5 (äußerst) kodiert und summiert, woraus sich ein Höchstwert von 230 und ein Minimum von 46 ergibt. Je höher die Zahl ist, umso besser ist das psychische Wohlbefinden. In den Überprüfungen der Hypothesen werden die Mittelwerte der Summe gerechnet. Die Beantwortungszeit beträgt 15 bis 20 Minuten. Die interne Konsistenz betrug in dieser Untersuchung Cronbach's Alpha $=.912$.

\subsubsection{Zur Analyse des Prozesses}

\subsubsection{Verlaufsentwicklung der Depression während des Programms: BDI}

Zur Analyse der Verlaufsentwicklung der Depression bei der Versuchsgruppe werden die Mittelwerte und Standardabweichungen sowie Cut-Off-Werte BDI (Becks Depression Inventory) eingesetzt, um ein klinisch bedeutsames Depressionsniveau zu interpretieren.

\subsubsection{Das hilfreichste Erlebnis (IHE)}

Diese Methode wurde von der Autorin mit dem Zweck der Erfassung dessen, woran sich jeder Teilnehmer nach jeder Sitzung als das hilfreichste Erlebnis erinnert, entwickelt. Jeder Teilnehmer beantwortet frei auf dem Papier die folgende Frage: Was ist für Sie in der Sitzung das Ereignis oder das Erlebnis, das Sie im Nachhinein als am hilfreichsten in dieser Sitzung empfinden? 1. Beschreiben Sie bitte die Situation, Ihre Gefühle, körperliche Reaktion oder Gedanken in dieser Situation. 2. Benennen Sie bitte das Erlebnis mit einem Satz. Dieses Instrument wird nach jeder Sitzung jedem Teilnehmer gegeben.

Außerdem ist das Instrument mit anderen Zielen verwandt. Jeder Teilnehmer hat dabei die Chance, beim Schreiben auf die Sitzung zurückzuschauen und sie für sich zusammenzufassen, damit die Therapie für jeden effektiv wird. Für den Therapeuten ist das Instrument hilfreich, um zu wissen, wie die Sitzung gerade bei den Teilnehmern angekommen ist. 


\section{Forschungsmethode}

\subsection{Messzeitpunkte}

Der Erhebungsablauf wird in Tabelle 7.11 anschaulich dargestellt.

Tab. 7.11: Messzeitpunkte

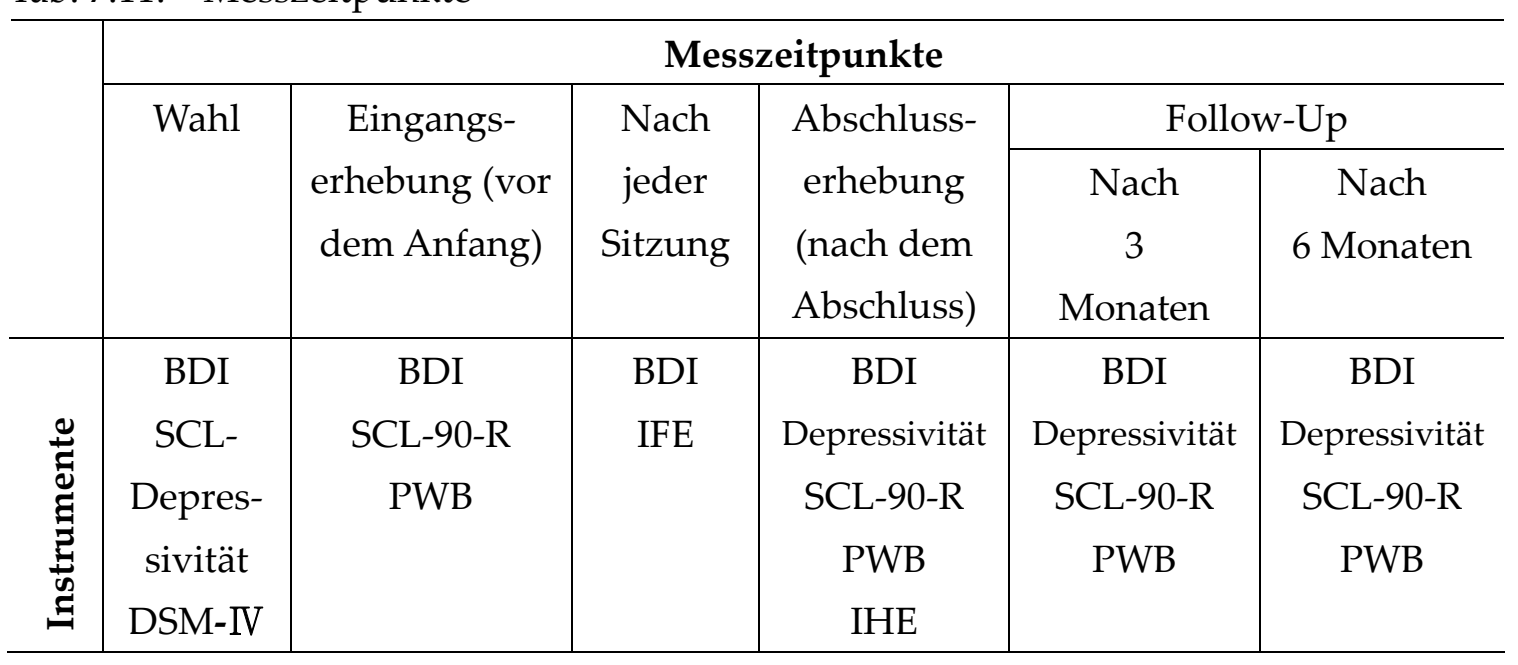

\subsection{Methode der Datenanalyse}

\subsubsection{Auswahl der statistischen Verfahren}

Zur Überprüfung der Hypothesen wird die Auswahl der statistischen Verfahren nach Drimm und Yarnold (2000) entsprechend getroffen. In der vorliegenden Arbeit geht es darum, zu prüfen, ob das Programm GPD-J im Vergleich zwischen Versuchs- und Kontrollgruppe für die Linderung der depressiven Symptomatik und auch weiterer allgemeiner psychischer Beschwerden und die Verbesserung psychischen Wohlbefindens wirksam ist. Die Auswertung erfolgt mit Hilfe des varianzanalytischen Messwiederholungsmodells in Gruppenvergleichen. Dieses erlaubt die Überprüfung der Effekte innerhalb der beiden Gruppen und zwischen ihnen. Bei der Varianzanalyse mit Messwiederholung wird eine Stichprobe mehrmals in Bezug auf ein bestimmtes Merkmal erfasst. Es handelt sich daher um einen „multivarianten“ Ansatz mit mehreren abhängigen Variablen (V1: Depressionswert vor Beginn einer Psychotherapie, V2: nach der Therapie, V3: nach 3 Monaten, V4: nach 6 Monaten) in der Versuchs- und Kontrollgruppe. Im Falle nur zweier Messungen ist die einfaktorielle Varianzanalyse mit dem t-Test für abhängige Stichproben identisch. Ein wesentlicher Vorteil von Messwiederholungsdesigns liegt in der Kontrolle von interindividuellen Unterschieden: Trug die individuelle Messwertvariation im Falle der Varianzanalyse 


\section{Forschungsmethode}

ohne Messwiederholung zur Fehlervarianz bei, können diese Unterschiede bei der wiederholten Messung auf den (zufälligen) Faktor der Versuchsperson zurückgeführt und aus der Fehlervarianz herausgerechnet werden (Leonhart, 2001). Die Gefahr der statistischen Analyse mit Differenzmaßen ist die fragliche Interpretierbarkeit bei unterschiedlicher psychometrischer Qualität der Instrumente. Dieses Problem umgeht man mit Messwiederholungsdesigns (Weinfurt 2000). Aus diesem Grund wird die Varianzanalyse mit Messwiederholung (Repeated Measures Analysis of Variance) zur Prüfung der Wirksamkeit des Programms mit den Variablen Zeit (prä, post, 3 und 6 Monate) x Bedingung (Prävention GPD-J vs. Kontrolle) verwendet.

Zunächst werden zur Überprüfung der Homogenität der beiden Gruppen gepaarte tTests zum Messzeitpunkt der Eingangserhebung mit allen Variablen BDI, SCL-RDepressivität, SCL-R-90 Mittelwerte und PWB verwendet.

Zur Überprüfung der im Kapitel 5 ausgeführten Hypothesen wurde die Varianzanalyse mit Messwiederholung (Repeated Measures Analysis of Variance) mit allen abhängigen Variablen BDI, SCL-R-Depressivität, SCL-R-9 und PWB im Vergleich der beiden Gruppen zum Zeitpunkt Prä und Post durchgeführt (Hypothese 1).

Dabei wurden alle abhängigen Variablen BDI, SCL-R-Depressivität, SCL-R-90 und PWB in der Varianzanalyse mit Messwiederholung (Repeated Measures Analysis of Variance) mit dem Faktor "Gruppe“ im Vergleich post, drei und sechs Monate nach der Beendigung des Programms zusammengefasst (Hypothese 2).

Für alle Hypothesen gilt das 0,05\%-Signifikanzniveau. Die Datenauswertung erfolgt mit dem Programm SPSS (Version 13.0).

\subsubsection{Zur Untersuchung des Therapieprozesses mit dem hilfreichsten Erlebnis}

Jede Teilnehmerin beschrieb nach jeder Sitzung das hilfreichste Erlebnis mit den Fragen, nach denen die Teilnehmerinnen frei ihre Erfahrung schreiben können. Die beschriebenen hilfreichsten Erlebnisse wurden der schrittweise untersucht. Erstens wurden sie nach der Bewertung der drei Personen, eine Ph. D. Studentin und zwei Psychologinnen analysiert, bewertet und sortiert. Diese Personen hatten vor der Bewertung fünf Stunden mit dem Kategoriensystem (vgl. 8.3.2.4) geübt. Bei der Analyse wurde das Videoband jeder Sitzung genutzt. Sie diskutierten über ihre Bewertungen, wenn diese nicht übereinstimmten. Nach dem Ende des Programms wurden Interviews mit jeder Teilnehmerin durchgeführt und nachgefragt. Darüber hinaus hat die Autorin die Erlebnisse schrittweise analysiert, um die Faktoren herauszufinden, die sich für die Teilnehmer am hilfreichsten erwiesen haben. 


\section{Ergebnisse}

\section{Ergebnisse}

\subsection{Homogenität der Gruppen}

Von den 44 Schülerinnen, die an der Untersuchung teilnehmen, gehören 22 Schülerinnen zur Präventionsgruppe und 22 zur Kontrollgruppe. Betrachtet man die Ausgangswerte der beiden Gruppen, bietet sich ein weitgehend homogenes Bild (vgl. Tab. 8.1).

Alle Teilnehmerinnen sind zwischen 16 und 18 Jahre alt. Das Durchschnittalter beträgt bei der GPD-J-Gruppe 16,91, die Standardabweichung beträgt SD =0,750. Bei der Kontrollgruppe ist das mittlere Alter 16,95, die Standardabweichung liegt bei SD = 0,785. Die Mittelwerte des Alters von beiden Gruppen erweisen sich bei einem t-Test als statistisch nicht signifikant unterschiedlich $(t=, 196 ; p=, 845>0,05)$.

Der t-Test zeigt, dass die beiden Gruppen in keinem Bereich signifikante Unterschiede aufweisen (vgl. Tabelle 8.1). Ein auffälliges Ergebnis ist die allgemeine Symptombelastung (SCL-90-R): Die Versuchsgruppe berichtet mehr allgemeine Symptombelastungen als die Kontrollgruppe. Aber das Ergebnis bei GSI $(p=, 053>$ 0,05) zeigt statistisch keine Unterschiede zwischen beiden Gruppen. Die Ausgangswerte depressiver Symptome (BDI) $(\mathrm{t}=-, 448, p=0,657)$, der Depressivität (SCL-90-R) $(t=-, 038, p=0,970)$ und des psychischen Wohlbefindens (PWB) $(t=-, 186, p$ $=0,864$ ) weisen keine Unterschiede auf.

Tab. 8.1: Mittelwerte zum ersten Messzeitpunkt beider Gruppen (Versuchs- und Kontrollgruppe) (t-Test mit unabhängigen Stichproben)

\begin{tabular}{|c|c|c|c|c|c|c|c|}
\hline & \multicolumn{2}{|c|}{$\begin{array}{c}\text { Versuchsgruppe } \\
\qquad \mathrm{N}=\mathbf{2 2}\end{array}$} & \multicolumn{2}{|c|}{$\begin{array}{l}\text { Kontrollgruppe } \\
\qquad \mathrm{N}=22\end{array}$} & \multicolumn{3}{|c|}{ t-Test } \\
\hline & $\mathbf{M}$ & SD & $\mathbf{M}$ & SD & $t$ & $d f$ & $\begin{array}{c}p \\
\text { (2-seitig) }\end{array}$ \\
\hline Alter & 16,91 & ,750 & 16,95 & ,785 & $-0,196$ & 42 & 0,845 \\
\hline BDI & 29,00 & 4,140 & 29,54 & 3,936 & $-0,448$ & 42 & 0,657 \\
\hline Deprssivität-SCL & 3,003 & ,314 & 3,007 & 294 & $-0,038$ & 42 & 0,970 \\
\hline SCL-90-R(GSI) & 1,482 & ,274 & 1,364 & 273 & $-0,781$ & 42 & 0,053 \\
\hline PWB & 1,722 & 098 & 1,716 & 132 & $-0,186$ & 42 & 0,854 \\
\hline
\end{tabular}




\section{Ergebnisse}

\subsection{Wirksamkeit des Programms}

\subsubsection{Prä-Post-Vergleich}

\subsubsection{Prä-Post-Vergleich der depressiven Symptome}

Die Varianzanalyse mit Messwiederholung zeigt einen signifikanten Effekt von der Bedingung "Gruppe" in den Variablen "BDI" $(F=188,086, p<$,001) und "Depressivität" $(F=330,196, p<, 001)$ und einen signifikanten Haupteffekt von "Zeit" in den Variablen „BDI" (F= 449,499; $p<$,001) und „Depressivität" ( F = 1903,817; $p<, 001)$. In Tabelle 8.2 werden Signifikanzen für den Faktor Zeit (Messwiederholungsfaktor) und den Interaktionsfaktor (Zeit $x$ Bedingung) wiedergegeben. Es bestätigen sich hier die signifikanten Messeffekte Zeit (Prä vs. Post) x Bedingung. Die Effekte zwischen "Zeit" und „Bedingung“ sind mit einem F-Wert ( $F=$ 542,529; $p<, 001)$ bei BDI, einem F-Wert $(F=2131,565 ; p<, 001)$ bei Depressivität (SCL90-R) hoch signifikant. Abbildung 1 und 2 zeigen positive Veränderungen depressiver Symptome im Vergleich der Mittelwerte BDI und der Depressivitätsskala der SCL-90-R in der Versuchs- und Kontrollgruppe. Bei "depressiven Symptomen (BDI)“ sowie „Depressivität (SCL-90-R)“ zeigt die Präventionsgruppe eine starke Verbesserung, während die Kontrollgruppe eine leichte Verschlechterung zeigt.

Tab. 8.2: Prä-Post-Vergleich der depressiven Symptome

\begin{tabular}{|c|c|c|c|c|c|c|c|}
\hline & $\begin{array}{l}\text { Subjekt- } \\
\text { effekte }\end{array}$ & Quelle & $\begin{array}{c}\text { Quadratsumme } \\
\text { von Typ III }\end{array}$ & $d f$ & $\begin{array}{l}\text { Mittel der } \\
\text { Quadrate }\end{array}$ & $F$ & $P$ \\
\hline \multirow[t]{6}{*}{ BDI } & \multirow[t]{4}{*}{ Innerhalb } & Zeit & 2921,011 & 1 & 2921,011 & 449,499 & , 000 \\
\hline & & Zeit $x$ & 3525,557 & 1 & 3525,557 & 542,529 & , 000 \\
\hline & & Gruppe & & & & & \\
\hline & & Fehler(Zeit) & 272,932 & 42 & 6,498 & & \\
\hline & \multirow[t]{2}{*}{ Zwischen } & Gruppe & 3835,920 & 1 & 3835,920 & 188,086 & , 000 \\
\hline & & Fehler & 856,568 & 42 & 20,394 & & \\
\hline \multirow{6}{*}{$\begin{array}{l}\text { Dep- } \\
\text { ress- } \\
\text { vität }\end{array}$} & \multirow[t]{4}{*}{ Innerhalb } & Zeit & 38,532 & 1 & 38,532 & 1903,817 & , 000 \\
\hline & & Zeit $x$ & 43,142 & 1 & 43,142 & 2131,565 & ,000 \\
\hline & & Gruppe & & & & & \\
\hline & & Fehler(Zeit) & ,850 & 42 & ,020 & & \\
\hline & \multirow[t]{2}{*}{ Zwischen } & Gruppe & 43,357 & 1 & 43,357 & 330,196 & ,000 \\
\hline & & Fehler & 5,515 & 42 & 131 & & \\
\hline
\end{tabular}




\section{Ergebnisse}

Abbildung 8.1 und 8.2 zeigen positive Veränderungen bei den depressiven Symptomen im Vergleich der Mittelwerte BDI und Depressivitätsskala der SCL-90-R in der Versuchs- und Kontrollgruppe.

Abb. 8.1: Prä-Post-Vergleich der Mittelwerte des Skalenwerts BDI in beiden Gruppen.

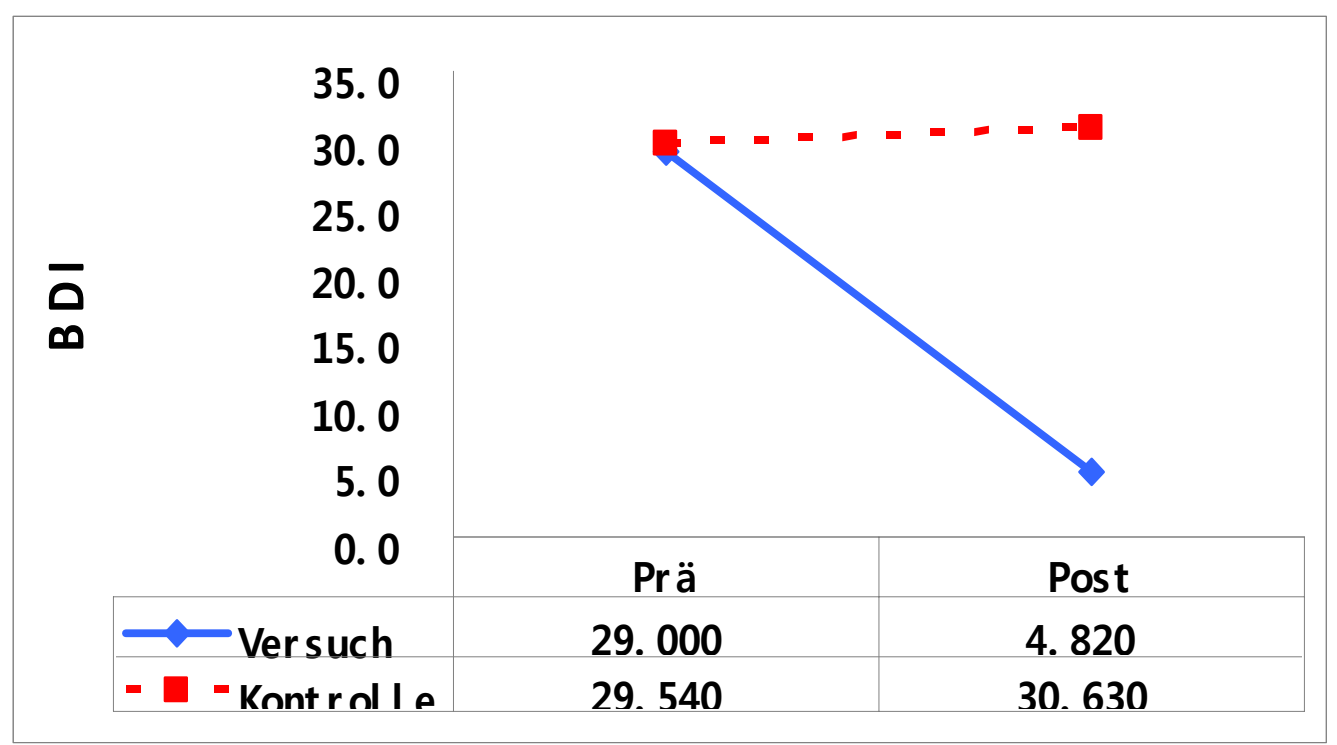

Abb. 8.2: Prä-Post-Vergleich der Mittelwerte der „Depressivität“ der SCL-90-R in beiden Gruppen.

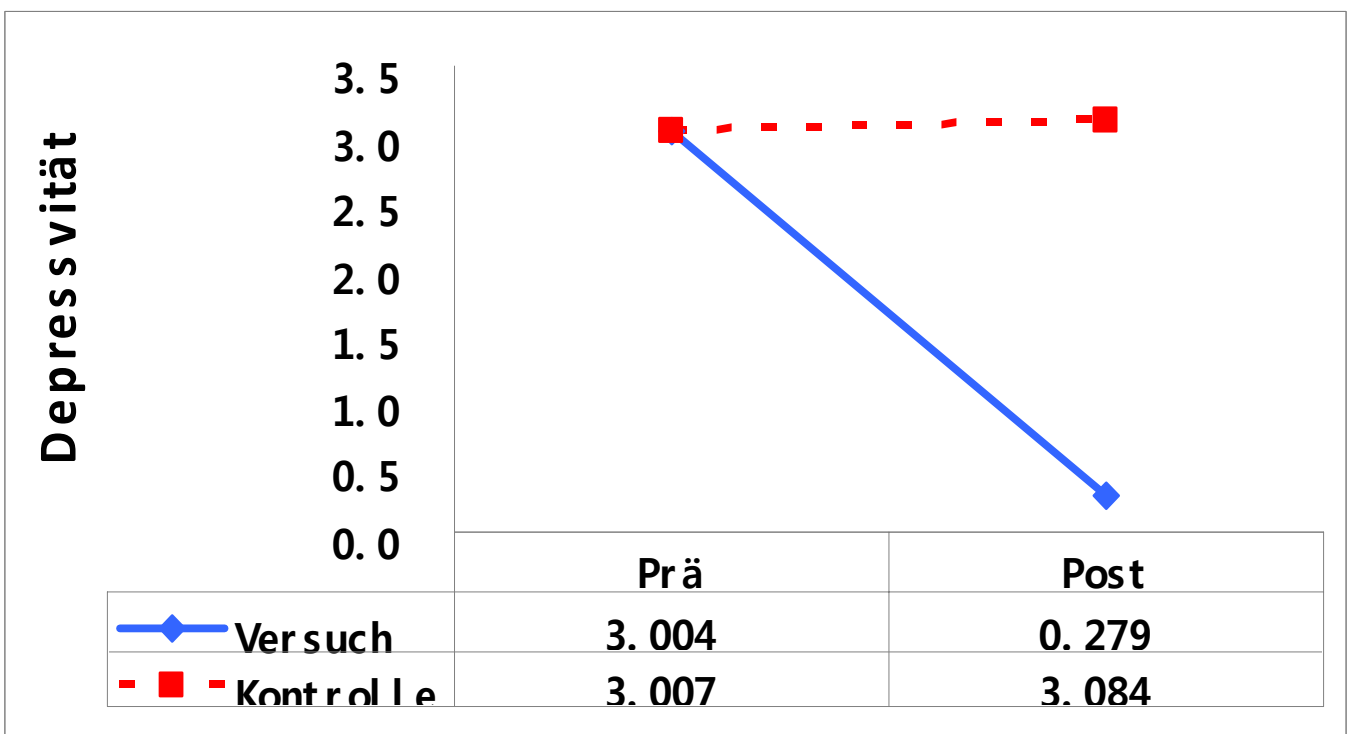




\section{Ergebnisse}

\subsubsection{Prä-Post-Vergleich allgemeiner psychischer Beschwerden}

Die Veränderungsmessung der allgemeinen psychischen Beschwerden erfolgte zunächst über den Vergleich der Gesamtwerte der beiden Variablen zwischen Eingangs- und Abschlusserhebung des Programms. Die Skalen der SCL-90-R messen die subjektive Beeinträchtigung eines Teilnehmers durch psychische Symptome. Eine Zunahme der Beeinträchtigung drückt sich in einer Erhöhung der Skalenwerte der SCL-90-R aus. Die Abnahme der Beeinträchtigung wird als positiver Indikator für einen Therapieerfolg gesehen.

Die Varianzanalyse mit Messwiederholung zeigt einen Haupteffekt der Bedingung (GPD-J vs. Kontrolle) ( $F=137,895 ; p<, 001)$ und einen signifikanten Effekt von der "Zeit" (F =457,009; $p<, 001)$ auf die Variable „allgemeine psychische Beschwerden“. In Tabelle 8.3 werden nun Signifikanzen für den Faktor Zeit (Messwiederholungsfaktor) und den Interaktionsfaktor (Zeit $x$ Bedingung) angegeben. Es bestätigt sich hier der signifikante Messwiederholungsfaktor Zeit (Prä vs. Post) x Bedingung (GPD-J vs. Kontrolle). Der Effekte zwischen "Zeit" und "Bedingung" hat einen F-Wert von $F=$ $625,224(p<, 001)$. Bei den allgemeinen psychischen Beschwerden zeigt die Präventionsgruppe eine starke Verbesserung, während die Kontrollgruppe eine leichte Verschlechterung zeigt. In Abbildung 8.3 ist eine positive Veränderung allgemeiner psychischer Beschwerden im Vergleich der Mittelwerte GSI (SCL-90-R) in der Versuchs- und Kontrollgruppe zu erkennen.

Tab. 8.3: Prä-Post-Vergleich allgemeiner psychischer Beschwerden in beiden Gruppen.

\begin{tabular}{ccccccc}
\hline $\begin{array}{c}\text { Subjekt- } \\
\text { effekte }\end{array}$ & Quelle & $\begin{array}{c}\text { Quadratsumme } \\
\text { von Typ II }\end{array}$ & $d f$ & $\begin{array}{c}\text { Mittel der } \\
\text { Quadrate }\end{array}$ & $\boldsymbol{F}$ & $\boldsymbol{P}$ \\
\hline Innerhalb & Zeit & 10,350 & 1 & 10,350 & 457,009 &, 000 \\
\cline { 2 - 7 } & $\begin{array}{c}\text { Zeit } \mathbf{x} \\
\text { Gruppe }\end{array}$ & 14,160 & 1 & 14,160 & 625,224 &, 000 \\
\cline { 2 - 7 } & $\begin{array}{c}\text { Fehler } \\
\text { (Zeit) }\end{array}$ &, 951 & 42 &, 023 & & \\
\hline Zwischen & Gruppe & 11,973 & 1 & 11,973 & 137,895 &, 000 \\
\cline { 2 - 8 } & Fehler & 3,647 & 42 &, 087 & & \\
\hline
\end{tabular}




\section{Ergebnisse}

Abb. 8.3: Prä-Post Vergleich der Mittelwerte des Skalenwerts „GSI(SCL-90-R)“ in beiden Gruppen.

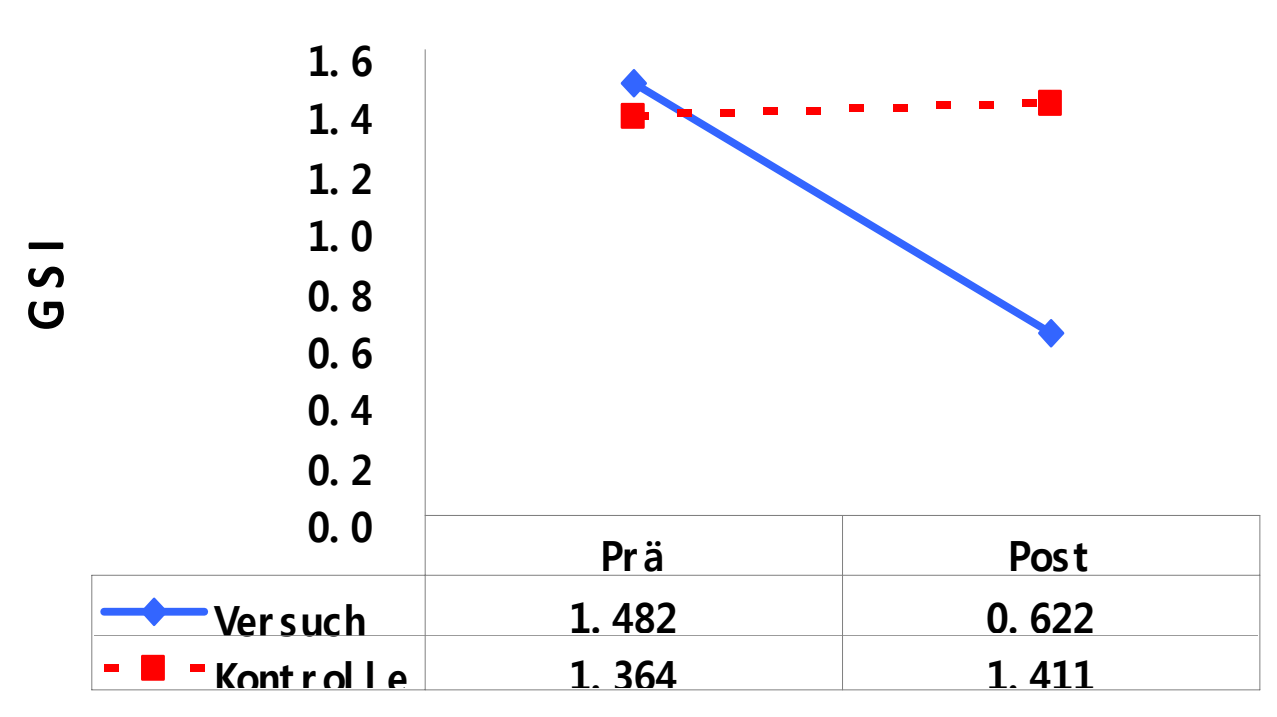

Tabelle 8.4 zeigt die Mittelwerte und Standardabweichungen der Subskalen der SCL90-R in beiden Gruppen im Vergleich zum Ausganspunkt und nach der Beendigung des Programms. In der Tabelle sind die Mittelwerte der gesunden Normierungsstichproben dargestellt. Der Prä-Post-Mittelwertvergleich der duchschnittlichen Rohwerte zeigt auf allen Skalen sowie bei dem Gesamtwert einen signifikanten Unterschied zwischen den Eingangs- und Abschlusswerten. Da die Varianzanalyse mit Messwiederholung bei der Variable GSI im Prä-Post-Vergleich signifikant ist, so dass die Präventionsgruppe positive Veränderung nach der Beendigung des Programms aufweist, wurde ein gepaarter t-Test mit Mittelwerten und Standardabweichungen der SCL-Skalen in der Präventionsgruppe durchgeführt, um die Wirkung zu bestätigen.

Die größten Mittelwertdifferenzen bei den psychischen Beschwerden wurden in der Präventionsgruppe auf der Skala „Depressivität" (Prä $M=3,004 ;$ Post $M=, 279 ; t=$ 48,396, $p<, 001)$ erreicht. Die signifikanten Mittelwerte wurden für die Skalen "Somatisierung" (Prä $M=2,076$; Post $M=$,526; $t=17,303, p<, 001$ ), „Interpersonale Unsicherheit" (Prä $M=2,287$; Post $M=, 686 ; t=7,370, p<, 001$ ), „Feindseligkeit“ (Prä $M$ = 1,341; Post $M=, 397 ; t=5,789, p<, 001$ ), "Zwanghaftigkeit" (Prä $M=1,414 ;$ Post $M$ $=, 323 ; t=5,765, p<, 001$ ) und "Ängstlichkeit" (Prä $M=1,636$; Post $M=, 900 ; t=4,213$, $p<, 001)$ in der Präventionsgruppe ermittelt. Die Skalenmittelwerte der Abschlusserhebung haben sich deutlich denen der gesunden Normalstichproben angenähert oder fallen sogar niedriger aus, während die Mittelwertdifferenzen in der 


\section{Ergebnisse}

Kontrollgruppe nicht signifikant sind. Die Mittelwerte auf den Subskalen „Phobische Angst", „Paranoides Denken“ und "Psychotizismus" zeigen keine signifikante Veränderung in beiden Gruppen.

Tab. 8.4: Prä-Post-Vergleich der Mittelwerte und Standardabweichungen der Subskalen der SCL-90-R in beiden Gruppen

\begin{tabular}{|c|c|c|c|c|c|c|c|c|c|c|}
\hline \multirow{3}{*}{$\begin{array}{c}\text { SCL-90-R } \\
\text { Skalen }\end{array}$} & \multirow{3}{*}{ MG } & \multicolumn{5}{|c|}{ GSPD-J } & \multicolumn{4}{|c|}{ Kontrollgruppe } \\
\hline & & \multicolumn{2}{|c|}{$\mathbf{M}$} & \multicolumn{2}{|c|}{ SD } & \multirow[t]{2}{*}{$t$} & \multicolumn{2}{|c|}{$\mathbf{M}$} & \multicolumn{2}{|c|}{ SD } \\
\hline & & Prä & Post & Prä & Post & & Prä & Post & Prä & Post \\
\hline Somatisierung & 0,77 & 2,076 & 205 & ,526 & 121 & $17,303^{*}$ & 1,849 & 1,955 & 351 & 399 \\
\hline Zwanghaftigkeit & 1,26 & 1,414 & 818 & .323 & ,289 & $5,765^{*}$ & 1,200 & 1,45 & ,264 & ,252 \\
\hline Interpersonale- & 1,23 & 2,287 & 117 & ,686 & ,323 & $7,370^{*}$ & 1,874 & 1,919 & 610 & ,569 \\
\hline \multicolumn{11}{|l|}{ Unsicherheit } \\
\hline Depressiviät & 1,08 & 3,004 & ,279 &, 314 & ,222 & $48,396^{*}$ & 3,007 & 3,084 & ,294 & 263 \\
\hline Ängstlichkeit & 0,93 & 1,636 & ,900 & ,703 & 245 & $4,213^{*}$ & 1,500 & 1,550 & ,679 & 645 \\
\hline Feindseligkeit & 0,92 & 1,341 & ,697 & ,397 & ,271 & $5,89^{*}$ & 1,174 & 1,007 & ,495 & ,335 \\
\hline Phobische Angst & 0,56 &, 539 & ,493 & ,223 & ,226 &, 87 & ,642 & ,707 & ,379 & ,255 \\
\hline ParanoidesDenken & 0,77 &, 583 & 469 & ,275 & ,244 & 1,373 &, 575 &, 554 & ,211 & ,242 \\
\hline Psychotizismus & 0,68 & 464 & 391 & 199 & 185 & 1,391 & 450 & 477 & 213 & 222 \\
\hline
\end{tabular}

Anmerkung: MG = gesunde Normierungsstichprobe, weibliche Jugendliche 16 bis 18 Jahre alt aus Korea (Kim et al.,1984, N=1031. Siehe. Tabelle 7.8). $\quad * p<$, oo1.

\subsubsection{Prä-Post-Vergleich des psychischen Wohlbefindens}

Die Veränderungsmessung des psychischen Wohlbefindens erfolgte zunächst über den Vergleich der Gesamtwerte zwischen Eingangserhebung vor und Abschlußerhebung nach dem Programm. Die Skalen der PWB messen das subjektive Wohlbefinden eines Teilnehmers. Eine Zunahme des Wohlbefindens drückt sich in einer Erhöhung der Skalenwerte der SCL-90-R aus. Die Zunahme des Wohlbefindens wird als positiver Indikator für einen Therapieerfolg gewertet.

Die Varianzanalyse mit Messwiederholung zeigt einen Haupteffekt der Bedingung (GPD-J vs. Kontrolle) ( $F=1348,876, p<, 001)$ und einen signifikanten Effekt von der "Zeit" ( $F=2714,049 ; p<, 001)$ auf die Variable „allgemeine psychische Beschwerden". In der Tabelle 8.5 werden nun Signifikanzen für den Faktor Zeit (Messwiederholungsfaktor) und den Interaktionsfaktor (Zeit $x$ Bedingung) wiedergegeben. Es bestätigt sich hier ein signifikanter Effekt Zeit (Prä vs. Post) $x$ 
Bedingung (GPD-J vs. Kontrolle) ( $F=2734,946, p<0,001)$. Bei dem Effekt zwischen "Zeit" und „Bedingung" ergibt sich ein F-Wert von $F=2734,946(p<, 001)$ für die Variable „psychisches Wohlbefinden“. Bei psychischem Wohlbefinden zeigt die Präventionsgruppe eine starke Verbesserung, während die Kontrollgruppe nur eine leichte Verbesserung zeigt.

Tab. 8.5: Prä-Post-Vergleich des psychischen Wohlbefindens in beiden Gruppen.

\begin{tabular}{ccccccc}
\hline $\begin{array}{c}\text { Subjekt- } \\
\text { effekte }\end{array}$ & Quelle & $\begin{array}{c}\text { Quadratsumme } \\
\text { von Typ III }\end{array}$ & df & $\begin{array}{c}\text { Mittel der } \\
\text { Quadrate }\end{array}$ & $\boldsymbol{F}$ & $\boldsymbol{P}$ \\
\hline Innerhalb & Zeit & 23,279 & 1 & 23,279 & 2714,049 &, 000 \\
\cline { 2 - 7 } & $\begin{array}{c}\text { Zeit } \mathbf{x} \\
\text { Gruppe }\end{array}$ & 23,458 & 1 & 23,458 & 2734,946 &, 000 \\
& Fehler(Zeit) &, 360 & 42 &, 009 & & \\
\hline Zwischen & Gruppe & 23,728 & 1 & 23,728 & 1348,876 &, 000 \\
\cline { 2 - 7 } & Fehler &, 739 & 42 &, 018 & & \\
\hline
\end{tabular}

Abbildung 8.4 zeigt eine positive Veränderung des psychischen Wohlbefindens im Vergleich der Mittelwerte PWB der Versuchs- und Kontrollgruppe.

Abb. 8.4: Prä-Post Vergleich der Mittelwerte des Skalenwert „PWB“

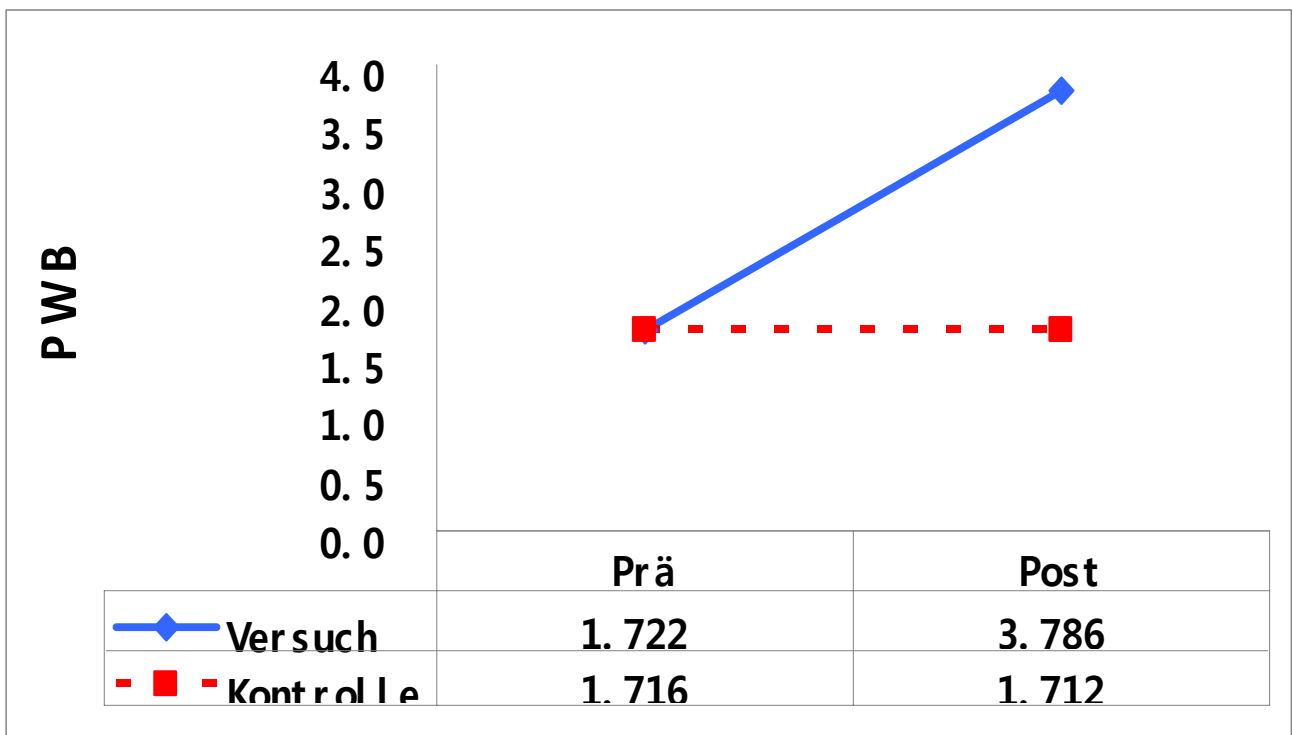

Tabelle 8.6 zeigt die Mittelwerte und Standardabweichungen der Subskalen des psychischen Wohlbefindens (PWB) in beiden Gruppen im Vergleich zum Ausganspunkt und nach der Beendigung des Programms. Der Prä-Post- 


\section{Ergebnisse}

Mittelwertvergleich der duchschnittlichen Rohwerte zeigt auf allen Skalen einen signifikanten Unterschied zwischen den Eingangs- und Abschlusswerten. Da sich die Varianzanalyse mit Messwiederholung der Variable Gesamtwerte PWBs im Prä-PostVergleich als signifikant erwiesen hat, so dass die Präventionsgruppe eine positive Veränderung nach der Beendigung des Programms aufweist, wurde ein gepaarter tTest mit Mittelwerten und Standardabweichungen der PWB-Skalen in der Präventionsgruppe durchgeführt, um die Wirkung zu bestätigen.

Die größten Mittelwertdifferenzen beim psychischen Wohlbefinden wurden in der Präventionsgruppe auf den Skalen „Lebenssinn“ (Prä $M=1,773$; Post $M=3,783$; $t$ = $37,814, p<, 001)$ ermittelt. Darüber hinaus wurden bei allen Skalen signifikante Mittelwertdifferenzen festgestellt: "Autonomie“ (Prä $M=1,635$; Post $M=3,528$; $t=$ 34,163, $p<, 001$ ), "Persönliches Wachstum“ (Prä $M=1,903$; Post $M=3,903 ; t=-33,723, p$ $<$,001), "Selbstakzeptanz" (Prä $M=1,596$; Post $M=3,983 ; t=-32,060, p<, 001)$, „Beherrschung der Welt" (Prä $M=1,761$; Post $M=3,471 ; t=-24,441, p<, 001$ ) und „Interpersonale Unsicherheit“ (Prä $M=1,668$; Post $M=3,948 ; t=-20,606, p<, 001$ ). Die Mittelwertdifferenzen der Kontrollgruppe waren hingegen nicht signifikant.

Tab. 8.6: Prä-Post-Vergleich der Mittelwerte und Standardabweichungen der Subskalen des psychischen Wohlbefindens in beiden Gruppen $\left({ }^{*} p<, 001\right.$. $)$

\begin{tabular}{|c|c|c|c|c|c|c|c|c|c|}
\hline \multirow{3}{*}{$\begin{array}{c}\text { PWB } \\
\text { Skalen }\end{array}$} & \multicolumn{4}{|c|}{ GSPD-J } & & \multicolumn{4}{|c|}{ Kontrollgruppe } \\
\hline & \multicolumn{2}{|c|}{$\mathbf{M}$} & \multicolumn{2}{|c|}{ SD } & \multirow[t]{2}{*}{$t$} & \multicolumn{2}{|c|}{$\mathbf{M}$} & \multicolumn{2}{|c|}{ SD } \\
\hline & Prä & Post & Prä & Post & & Prä & Post & Prä & Post \\
\hline Selbstakzeptanz & 1,600 & 3,983 & ,250 & ,212 & $-32,060^{*}$ & 1,740 & 1,671 & 189 & ,285 \\
\hline Positive Beziehung & 1,669 & 3,948 & 280 & 403 & $-20,606^{*}$ & 1,584 & 1,688 & ,264 & ,320 \\
\hline Autonomie & 1,631 & 3,528 & ,257 & ,200 & $-34,163^{*}$ & 1,665 & 1,676 & ,209 & ,227 \\
\hline Beherrschung der & 1,761 & 3,472 & 211 & ,234 & $-24,441^{*}$ & 1,915 & 1,886 & ,206 & ,270 \\
\hline \multicolumn{10}{|l|}{ Lebenswelt } \\
\hline Lebenssinn & 1,773 & 3,903 & ,205 & ,235 & $-37,814^{*}$ & 1,766 & 1,578 & ,205 & ,287 \\
\hline Persönliches Wachstum & 1,903 & 3,903 & ,234 & ,218 & $-33,723^{*}$ & 1,619 & 1,756 & ,248 & ,285 \\
\hline
\end{tabular}

\subsubsection{Follow-up-Vergleich}

Im Folgenden sollen Varianzanalysen dargestellt werden, die der Frage nachgehen, inwiefern die Teilnahme an dem Präventionsprogramm GPD-J nach drei und sechs Monaten auf der Ebene der Einzelvariablen positive Effekte im Vergleich mit der nicht 


\section{Ergebnisse}

behandelten Kontrollgruppe ergeben. Entsprechend ergibt sich folgendes Design: Zeit (Prä, Post, drei und sechs Monate danach) x Bedingung (Prävention GPD-J vs. Kontrolle).

\subsubsection{Follow-up-Vergleich der depressiven Symptome}

Die depressive Symptomatik, erhoben über BDI und SCL-Depressivität, nimmt über die Messzeitpunkte hinweg bei der Präventionsgruppe signifikant ab, während sich die Werte der Kontrollgruppe nicht signifikant verändern (Tabelle 8.7). Die Varianzanalyse mit Messwiederholung in der Variable „BDI“ zeigt einen signifikanten Haupteffekt von "Zeit" ( $F=219.516 ; p<, 001)$ und weiterhin einen signifikanten Effekt "Zeit x Gruppe" (F=280.568; $p<, 001)$. Es zeigt, dass dieser Effekt auf Unterschiede zwischen den Messzeitpunkten in der Präventionsbedingung GPD-J zurückzuführen ist, während die Unterschiede in der Kontrollgruppe nicht signifikant sind ( $F=460,449$; $p<, 001)$. Die Ergebnisse sind auch für die Variable SCL-Depressivität signifikant. Einen Haupteffekt von "Zeit" ( $F=246,013 ; p<, 001)$ und einen signifikanten Effekt "Zeit x Gruppe" $(F=228.588 ; p<, 001)$ ergibt die entsprechende Varianzanalyse mit Messwiederholung. Weiterhin ergibt sich ein signifikanter Effekt der Variable "Gruppe“ ( $F=487,117 ; p<, 001)$.

Tab. 8.7: Varianzanalyse mit Messwiederholung über vier Zeitpunkte für depressive Symptomatik

\begin{tabular}{|c|c|c|c|c|c|c|c|}
\hline & $\begin{array}{l}\text { Subjekt- } \\
\text { effekte }\end{array}$ & Quelle & $\begin{array}{c}\text { Quadratsumme } \\
\text { von Typ III }\end{array}$ & $d f$ & $\begin{array}{l}\text { Mittel der } \\
\text { Quadrate }\end{array}$ & $F$ & $P$ \\
\hline \multirow[t]{6}{*}{ BDI } & \multirow[t]{4}{*}{ Innerhalb } & Zeit & 3552409 & 3 & 1184,136 & 219,516 & ,000 \\
\hline & & Zeit $x$ & 4540,409 & 3 & 1513,470 & 280,568 & ,000 \\
\hline & & Gruppe & & & & & \\
\hline & & Fehler(Zeit) & 679,682 & 126 & 5,394 & & \\
\hline & \multirow[t]{2}{*}{ Zwischen } & Gruppe & 13969,455 & 1 & 82737,818 & 460,449 & ,000 \\
\hline & & Fehler & 1274,227 & 42 & 30,339 & & \\
\hline \multirow{6}{*}{$\begin{array}{l}\text { Dep- } \\
\text { ress- } \\
\text { vität }\end{array}$} & \multirow[t]{4}{*}{ Innerhalb } & Zeit & 57,618 & 3 & 19,206 & 246,013 & ,000 \\
\hline & & Zeit $x$ & 64,983 & 3 & 21,661 & 228,588 & ,000 \\
\hline & & Gruppe & & & & & \\
\hline & & Fehler(Zeit) & 3,746 & 126 & ,030 & & \\
\hline & \multirow[t]{2}{*}{ Zwischen } & Gruppe & 195,594 & 1 & 195,594 & 487,117 & ,000 \\
\hline & & Fehler & 5,524 & 42 & 132 & & \\
\hline
\end{tabular}




\section{Ergebnisse}

Die depressive Symptomatik ändert sich bei der Präventionsgruppe GPD-J im Laufe der Zeit bemerkenswert, während sich eine leichte Verschlechterung bei der Kontrollgruppe zeigt (Abbildung 8.5 und Abbildung 8.6). Bei der Versuchsgruppe erhöhen sich die Mittelwerte des BDI-Tests 3 Monate nach Ende des Programms und noch einmal nach weiteren 3 Monaten. Die Mittelwerte der SCL-Depressivität senken sich bei der Versuchsgruppe nach 3 Monaten nach dem Ende des Programms, aber sie erhöhen sich wieder nach weiteren 3 Monaten.

Abb. 8.5: Vergleich der Verlaufsentwicklung BDI in beiden Gruppen.

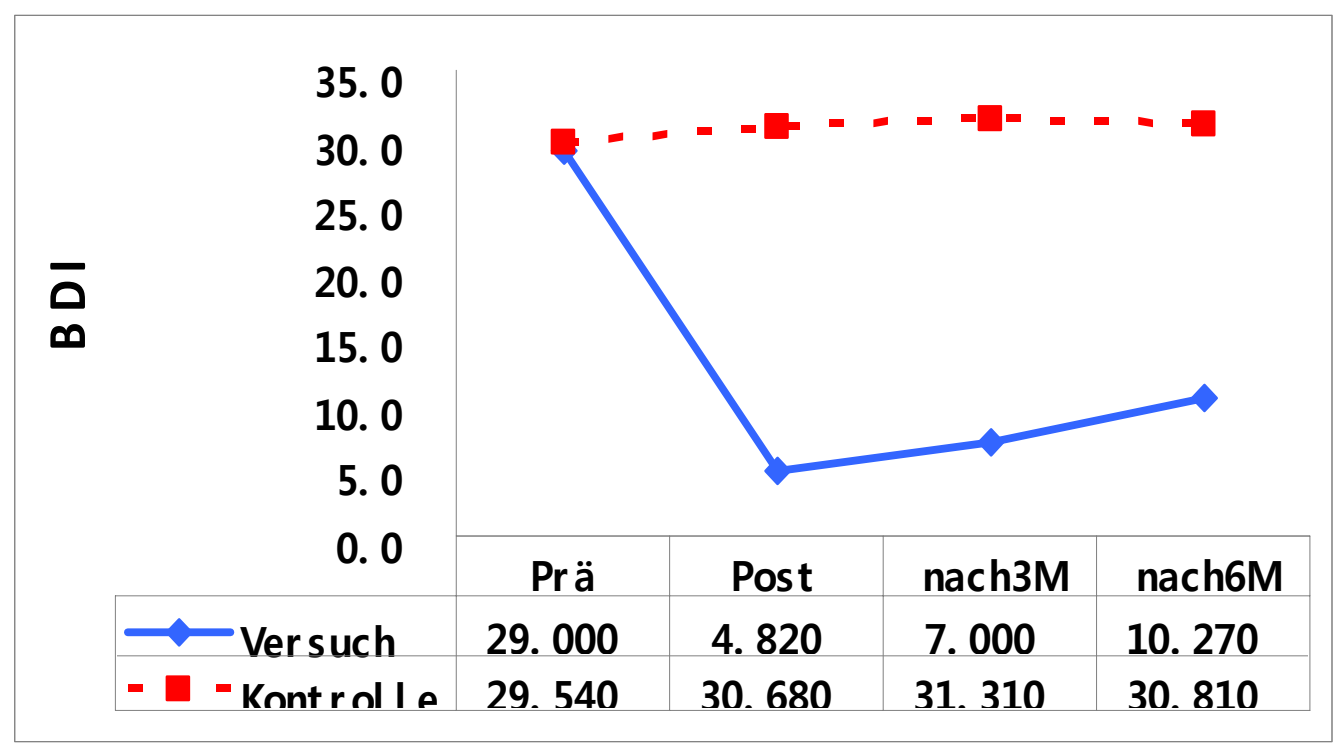

Abb. 8.6: Vergleich der Verlaufsentwicklung SCL-Depressivität in beiden Gruppen.

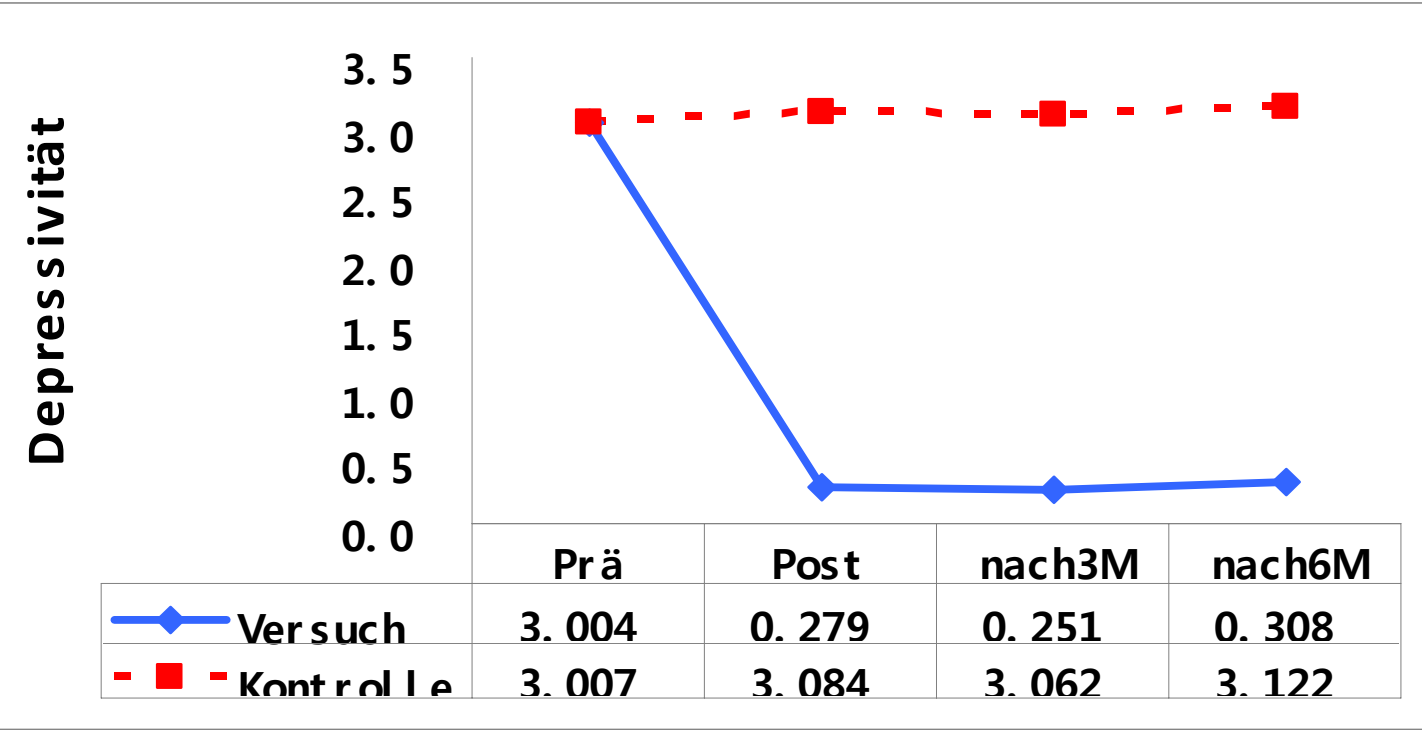




\section{Ergebnisse}

\subsubsection{Follow-up-Vergleich allgemeiner psychischer Beschwerden}

Allgemeine psychische Beschwerden, erhoben über GSI von SCL-90-R, nehmen über die Messzeitpunkte hinweg bei der Präventionsgruppe signifikant ab, während sich die Werte der Kontrollgruppe nicht signifikant verändern (Tabelle 8.8).

Die Varianzanalyse mit Messwiederholung der Variable GSI (SCL-90-R) zeigt einen signifikanten Haupteffekt "Zeit" $(F=315,184 ; p<, 001)$ und weiterhin einen signifikanten Effekt "Zeit x Gruppe“ ( $F=470,907 ; \quad p<, 001)$. Es zeigt, dass dieser

Effekt auf Unterschiede zwischen den Messzeitpunkten in den Präventionsbedingungen GPD-J zurückzuführen ist, während die Unterschiede in der Kontrollgruppe nicht signifikant sind. Weiterhin ergibt sich ein signifikanter Unterschied zwischen den Gruppen zu den Messzeitpunkten nach drei und sechs Monaten $(F=367,135 ; p<, 001)$.

Tab. 8.8: Varianzanalyse mit Messwiederholung über vier Zeitpukte für die Variable "allgemeine psychische Beschwerden"

\begin{tabular}{ccccccc}
\hline $\begin{array}{c}\text { Subjekt- } \\
\text { effekte }\end{array}$ & Quelle & $\begin{array}{c}\text { Quadratsumme } \\
\text { von Typ III }\end{array}$ & $d f$ & $\begin{array}{c}\text { Mittel der } \\
\text { Quadrate }\end{array}$ & $\boldsymbol{F}$ & $\boldsymbol{P}$ \\
\hline Innerhalb & Zeit & 13,958 & 3 & 4,653 & 315,184 &, 000 \\
\cline { 2 - 7 } & $\begin{array}{c}\text { Zeit x } \\
\text { Gruppe }\end{array}$ & 20,854 & 3 & 6,951 & 470,907 &, 000 \\
& $\begin{array}{c}\text { Fehler } \\
\text { (Zeit) }\end{array}$ & 1,860 & 126 &, 015 & & \\
\hline Zwischen & Gruppe & 55,733 & 1 & 55,733 & 367,135 &, 000 \\
\cline { 2 - 7 } & Fehler & 6,376 & 42 &, 152 & & \\
\hline
\end{tabular}

Die allgemeinen psychischen Beschwerden ändern sich bei der Präventionsgruppe GPD-J im Laufe der Zeit bemerkenswert positiv, während sich eine leichte Verschlechterung bei der Kontrollgruppe zeigt (Abbildung 8.7). 3 Monate nach dem Ende des Programms erhöhen sich bei der Versuchsgruppe die GSI-Mittelwerte und dies setzt sich nach weiteren 3 Monaten fort. 


\section{Ergebnisse}

Abb. 8.7: Vergleich der Verlaufsentwicklung „allgemeiner psychischer Beschwerden" in beiden Gruppen.

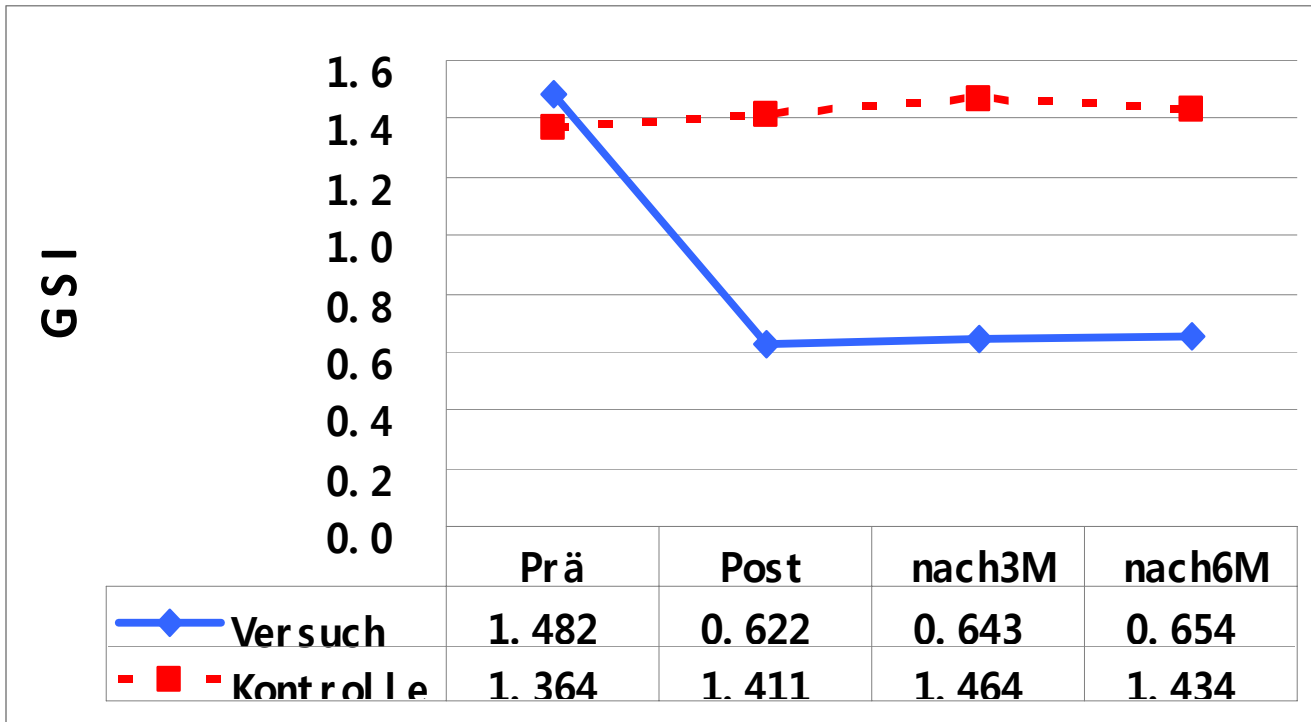

Darüber hinaus zeigt Tabelle 8.9 die Mittelwerte und Standardabweichung der Subskalen „allgemeine psychische Beschwerden“ sowie die Mittelwerte gesunder Normierungsstichproben.

Im Vergleich der Mittelwerte aller Skalen mit Normierungsstichproben zeigt die Versuchsgruppe den größten Abstand bei der Skala "Somatisierung“ ( $M(M G)=0,77$, nach 3 Monaten $M=0,264$, nach 6 Monaten $M=0,374$ ), während sich eine geringere Mittelwertdifferenz bei der Skala "Unsicherheit" $(M(M G)=1,23$, nach 3 Monaten = 1,144, nach 6 Monaten = 1,122) zeigt. Die Mittelwerte der Skala „Ängstlichkeit“ bei der Versuchsgruppe nach 3 bzw. nach 6 Monaten sind höher als bei den Normierungsstichproben. Bei der Kontrollgruppe sind die Mittelwerte fast aller Skalen höher als bei Normierungsstichproben im Vergleich prä, 3 bzw. 6 Monate. Die Mittelwerte der Skalen „Paranoides Denken“ und "Psychotizismus" in beiden Gruppen sind niedriger als die Normierungsstichproben zu allen Messzeitpunkten. 


\section{Ergebnisse}

Tab. 8.9: Follow-Up-Vergleich der Subskalen allgemeiner psychischen Beschwerden in beiden Gruppen.

\begin{tabular}{|c|c|c|c|c|c|c|c|c|c|c|}
\hline \multirow[t]{2}{*}{ Subskala } & \multirow[t]{2}{*}{ MG } & \multirow{2}{*}{$\begin{array}{c}\text { Grup } \\
\text { pen- } \\
\text { typ }\end{array}$} & \multicolumn{2}{|c|}{ Prä } & \multicolumn{2}{|c|}{ Post } & \multicolumn{2}{|c|}{$\begin{array}{c}\text { nach } \\
3 \text { Monaten }\end{array}$} & \multicolumn{2}{|c|}{$\begin{array}{c}\text { nach } \\
6 \text { Monaten }\end{array}$} \\
\hline & & & $\mathbf{M}$ & SD & $\mathbf{M}$ & SD & $\mathbf{M}$ & SD & $\mathbf{M}$ & SD \\
\hline Somatisie- & 0,77 & Vers & 2,075 & ,526 & ,204 & 121 & ,264 & 174 &, 374 & 179 \\
\hline rung & & Kont & 1,849 & ,351 & 1,955 & ,399 & 1,958 & ,363 & 2,041 & 416 \\
\hline Zwanghaftig & 1,26 & Vers & 1,413 & ,323 & 818 & ,288 & 940 & ,125 & ,790 & 248 \\
\hline -keit & & Kont & 1,200 & ,263 & 1,450 & ,252 & 1,740 & ,347 & 1,686 & ,352 \\
\hline \multirow[t]{2}{*}{ Unsicherheit } & 1,23 & Vers & 2,287 & 685 & 1,171 & ,322 & 1,144 & ,367 & 1,221 & 290 \\
\hline & & Kont & 1,873 & 609 & 1,919 &, 569 & 1,919 &, 516 & 1,878 & ,445 \\
\hline Ängstlich- & 0,93 & Vers & 1,636 & 703 & 900 & 244 & ,945 & 228 & 1,036 & ,315 \\
\hline keit & & Kont & 1,500 & 679 & 1,550 & 640 & 1,668 & 646 & 1,654 &, 539 \\
\hline Feindseling- & 0,92 & Vers & 1,340 & ,396 & 697 & 270 & ,727 & 301 & ,795 & ,280 \\
\hline keit & & Kont & 1,174 & 494 & 1,007 & ,335 & 1,143 & ,480 & 1,060 & 400 \\
\hline Phobische & 0,56 & Vers &, 539 & ,223 & ,492 & ,226 & 458 & ,219 &, 543 & 248 \\
\hline Angst & & Kont & ,642 & ,378 & ,707 & ,251 & ,674 & ,344 & ,733 & ,385 \\
\hline Paranoides & 0,77 & Vers & ,582 & ,275 & ,469 & ,244 & 492 & ,208 & ,438 & ,238 \\
\hline Denken & & Kon &, 575 & ,210 & ,533 & ,242 & ,568 & ,279 & ,584 & ,251 \\
\hline Psychotizis- & 0,68 & Vers & ,463 & 198 & ,390 & ,184 & ,377 & 174 & ,381 & ,162 \\
\hline mus & & Kont & ,450 & ,213 & ,477 & ,222 & 445, & 217 &, 450 & 197 \\
\hline
\end{tabular}

Anmerkung: MG1= gesunde Normierungsstichprobe: weibliche Jugendliche von 16 bis 18 Jahren, aus Korea (Kim et al.,1984, N=1031. siehe. Tabelle 7.8).

\subsubsection{Follow-up-Vergleich psychischen Wohlbefindens}

Psychisches Wohlbefinden, erhoben über PWB, nimmt über die Messzeitpunkte hinweg bei der Präventionsgruppe signifikant $\mathrm{zu}$, während es sich bei der Kontrollgruppe nicht signifikant verändert (Tabelle 8.10). Die Varianzanalyse mit Messwiederholung der Variable „psychisches Wohlbefinden“ zeigt einen signifikanten Haupteffekt der "Zeit" ( $F=189,797 ; p<, 001)$ und weiterhin einen signifikanten Effekt von "Zeit x Gruppe" $(F=166,701 ; \quad p<, 001)$. Es zeigt, dass dieser Effekt auf Unterschiede zwischen den Messzeitpunkten in der Präventionsbedingungen GPD-J zurückzuführen ist, während die Unterschiede in der Kontrollgruppe nicht signifikant sind. Weiterhin ergibt sich ein signifikanter Unterschied zwischen den Gruppen zu den Messzeitpunkten nach drei und sechs Monaten $(F=274,248 ; p<, 001)$. 


\section{Ergebnisse}

Psychisches Wohlbefinden ändert sich über die Präventionsgruppe im Laufe der Zeit bemerkenswert, während sich eine leichte Verschlechterung bei der Kontrollgruppe zeigt (Abbildung 8.8).

Tab. 8.10: Varianzanalyse mit Messwiederholung über vier Zeitpukte für "psychisches Wohlbefinden“"

\begin{tabular}{llccccc}
\hline $\begin{array}{l}\text { Subjekt- } \\
\text { effekte }\end{array}$ & Quelle & $\begin{array}{c}\text { Quadratsumme } \\
\text { von Typ II }\end{array}$ & $d f$ & $\begin{array}{c}\text { Mittel der } \\
\text { Quadrate }\end{array}$ & $\boldsymbol{F}$ & $\boldsymbol{P}$ \\
\hline Innerhalb & Zeit & 27,754 & 3 & 9,251 & 186,797 &, 000 \\
\cline { 2 - 7 } & $\begin{array}{l}\text { Zeit x } \\
\text { Gruppe }\end{array}$ & 27,240 & 3 & 9,080 & 166,701 &, 000 \\
& $\begin{array}{l}\text { Fehler } \\
\text { (Zeit) }\end{array}$ & 1,073 & 126 &, 039 & & \\
\hline Zwischen & Gruppe & 77,144 & 1 & 77,144 & 274,248 &, 000 \\
\cline { 2 - 7 } & Fehler & 1,182 & 42 &, 028 & & \\
\hline
\end{tabular}

Wie die Abbildung 8 zeigt, änderte sich „psychisches Wohlbefinden“ über die Versuchsgruppe im Lauf der Zeit deutlich, während sich bei der Kontrollgruppe eine Verschlechterung zeigt. Die Mittelwerte psychischen Wohlbefindens senken sich bei der Versuchsgruppe 3 Monate nach dem Ende des Programms und ebenso nach weiteren 3 Monaten.

Abb. 8.8: Vergleich der Verlaufsentwicklung „Psychisches Wohlbefinden“ in beiden Gruppen.

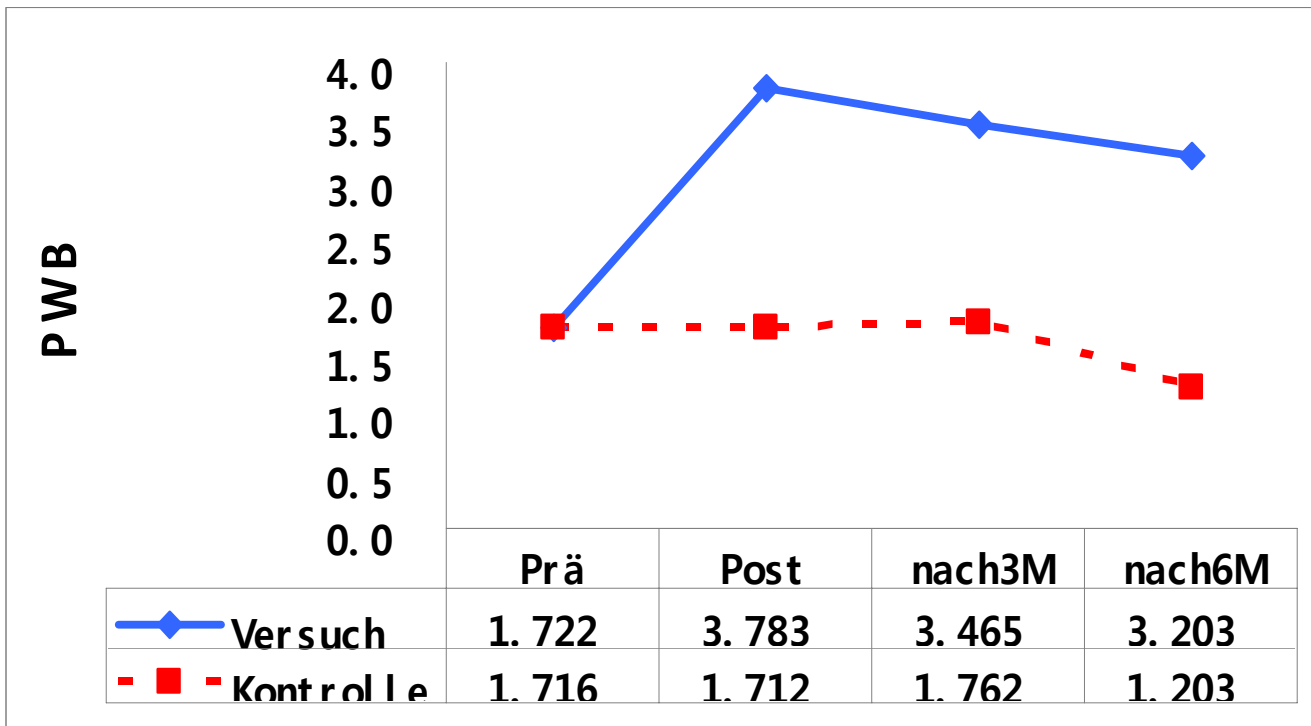




\section{Ergebnisse}

3 Monate nach dem Ende des Programms senken sich die Mittelwerte der Versuchsgruppe bei allen PWB-Subskalen. Dies setzt sich nach weiteren 3 Monaten, mit Ausnahme der Skala „Lebenssinn“, ebenso fort. Die Mittelwerte der Skala „Beherrschung der Welt“ änderten sich bei der Versuchsgruppe am geringsten im Vergleich mit den Veränderungen der anderen Skalen. Der Mittelwert der Skala „Lebenssinn“ senkt sich in beiden Gruppen gleich 3 Monate nach dem Ende des Programms (Tabelle 8. 11).

Tab. 8.11: Follow-Up-Vergleich der Subskalen des psychischen Wohlbefindens in beiden Gruppen.

\begin{tabular}{|c|c|c|c|c|c|c|c|c|c|}
\hline \multirow[t]{2}{*}{ Subskala } & \multirow[t]{2}{*}{$\begin{array}{l}\text { Grup- } \\
\text { entyp }\end{array}$} & \multicolumn{2}{|c|}{ Prä } & \multicolumn{2}{|c|}{ Post } & \multicolumn{2}{|c|}{$\begin{array}{c}\text { nach } \\
3 \text { Monaten }\end{array}$} & \multicolumn{2}{|c|}{$\begin{array}{c}\text { nach } \\
6 \text { Monaten }\end{array}$} \\
\hline & & $\mathbf{M}$ & SD & $\mathbf{M}$ & SD & $\mathbf{M}$ & SD & $\mathbf{M}$ & SD \\
\hline \multirow{2}{*}{$\begin{array}{l}\text { Selbstakzept- } \\
\text { anz }\end{array}$} & Vers & 1,596 & 249 & 3,983 & ,212 & 3,795 & 307 & 3,562 & 199 \\
\hline & Kont & 1,738 & 188 & 1,670 & ,284 & 1,625 & ,320 & 1,812 & 143 \\
\hline Positive & Vers & 1,668 & 280 & 3,948 & 403 & 3,539 & 341 & 3,409 & 312 \\
\hline Beziehung & Kont & 1,584 & ,264 & 1,688 & ,319 & 1,837 & ,254 & 1,643 & ,244 \\
\hline \multirow[t]{2}{*}{ Autonomie } & Vers & 1,635 & ,257 & 3,528 & 200 & 3,380 & 274 & 3,022 & ,331 \\
\hline & Kont & 1,664 & 209 & 1,676 & ,227 & 1,841 & 325 & 1,716 & 259 \\
\hline \multirow{2}{*}{$\begin{array}{l}\text { Beherrschung } \\
\text { der Welt }\end{array}$} & Vers & 1,761 & 210 & 3,471 & ,234 & 2,988 & 193 & 2,704 & 157 \\
\hline & Kont & 1,915 & 205 & 1,887 & 269 & 1,858 & 265 & 1,909 & 182 \\
\hline \multirow[t]{2}{*}{ Lebenssinn } & Vers & 1,773 & 205 & 3,903 & ,234 & 3,558 & 224 & 3,331 & 199 \\
\hline & Kont & 1,766 & 205 & 1,578 & 287 & 1,508 & 343 & 1,805 & 209 \\
\hline \multirow[t]{2}{*}{ Wachstum } & Vers & 1,903 & ,234 & 3,903 & ,217 & 3,551 & 295 & 3,233 & 219 \\
\hline & Kont & 1,619 & 248 & 1,756 & 285 & 1,705 & 312 & 1,705 & 269 \\
\hline
\end{tabular}

\subsection{Prozessanalyse}

\subsubsection{Entwicklung der „depressiven Symptome“ bei der Präventionsgruppe}

Obwohl ein klinisches Interview bei der Auswahl zur Teilnahme an dem Projekt durchgeführt wurde, wurden keine klinischen Interviews zum Abschluss durchgeführt, so dass keine Aussagen über klinische Diagnosen gemacht werden können. Als Hinweis auf ein klinisch bedeutsames Depressionsniveau können aber erhöhte Werte in Becks Depression Inventar (BDI) interpretiert werden. Um Depressionsverläufe während des Programms zu untersuchen, wurden die Werte von Becks 


\section{Ergebnisse}

Depressionsinventars nach jeder Sitzung von den Teilnehmerinnen erhoben. Aus Lee und Songs Studie (1991) ergab sich, dass man bei weiblichen Jugendlichen bei Werten zwischen 16 und 23 von subklinischer Depression spricht, während Werte ab 24 auf eine klinisch manifeste Depression hinweisen.

Die Anzahl der BDI-Werte bis 15 erhöhte sich nach der fünften Sitzung deutlich, und die Anzahl der Werte ab 24 sankt nach der ersten Sitzung. Ab der neunten Sitzung zeigen alle Teilnehmerinnen BDI-Werte bis 15.

In der Baseline-Erhebung war der BDI-Mittelwert 29,000 (SD = 4,410) und nach dem Ende des Programms 4,818 (SD = 6,727), nach drei Monaten 7,000 $(\mathrm{SD}=7,810)$ und nach sechs Monaten 10,272 (SD = 4,113) (vgl. Tabelle 8.12). Während des Programms zeigen die Mittelwerte nach der ersten Sitzung die größte Differenz $(M=22,546, S D=$ $5,942)$ im Vergleich zur Baseline $(M=29,000, S D=4,410)$. Die zweitgrößte Differenz folgt in der fünften Sitzung $(M=10,500, S D=3,335)$ im Vergleich zur vierten Sitzung $(\mathrm{M}=15,727, \mathrm{SD}=5,938)$.

Tab. 8.12: Entwicklung der depressiven Symptome bei der Präventionsgruppe(N=22)

\begin{tabular}{cccccc}
\hline \multirow{2}{*}{ Zeitverlauf } & \multicolumn{2}{c}{ Anzahl nach BDI-Cut-Off-Werte } & \multirow{2}{*}{ M } & SD \\
\cline { 2 - 4 } & bis 15 & $\mathbf{1 6 - 2 3}$ & über 24 & & \\
\hline Baseline & 0 & 0 & 22 & 29,000 & 4,410 \\
\hline nach 1. Sitzung & 3 & 9 & 10 & 22,546 & 5,942 \\
nach 2. Sitzung & 0 & 12 & 10 & 21,828 & 4,425 \\
nach 3. Sitzung & 7 & 9 & 6 & 17,500 & 6,981 \\
nach 4. Sitzung & 10 & 7 & 5 & 15,727 & 5,938 \\
nach 5. Sitzung & 16 & 4 & 2 & 10,500 & 3,335 \\
\hline nach 6. Sitzung & 17 & 4 & 1 & 11,546 & 2,220 \\
nach 7. Sitzung & 18 & 3 & 1 & 8,455 & 2,940 \\
nach 8. Sitzung & 19 & 2 & 0 & 6,136 & 2,077 \\
nach 9. Sitzung & 22 & 0 & 0 & 5,364 & 1,840 \\
nach 10. Sitzung & 22 & 0 & 0 & 4,818 & 6,727 \\
\hline nach 3 Monaten & 21 & 1 & 0 & 7,000 & 7,810 \\
\hline nach 6 Monaten & 20 & 2 & 0 & 10,272 & 4,113 \\
\hline
\end{tabular}

Wie sich in der Abbildung 8.9 zeigt, hat eine dramatische Veränderung der BDI-Werte von der vierten zur fünften Sitzung stattgefunden. In der vierten Sitzung waren die BDI-Werte 15,727 (SD = 5,938), dann 10, $500(S D=3,335)$ in der fünften Sitzung. Der geringste Unterschied bestand zwischen den BDI-Werte der zehnten Sitzung ( $M=4,818$, 


\section{Ergebnisse}

$\mathrm{SD}=6,727)$ und der neunten Sitzung $(\mathrm{M}=5,364, \mathrm{SD}=1,840)$. Dann erhöhen sich die BDI-Werte drei Monate $(M=7,000, S D=7,810)$ und sechs Monate $(M=10,272, S D=$ 4,113) nach dem Endes des Programms.

Abb. 8.9: Entwicklung der „depressiven Symptome“ bei der Präventionsgruppe

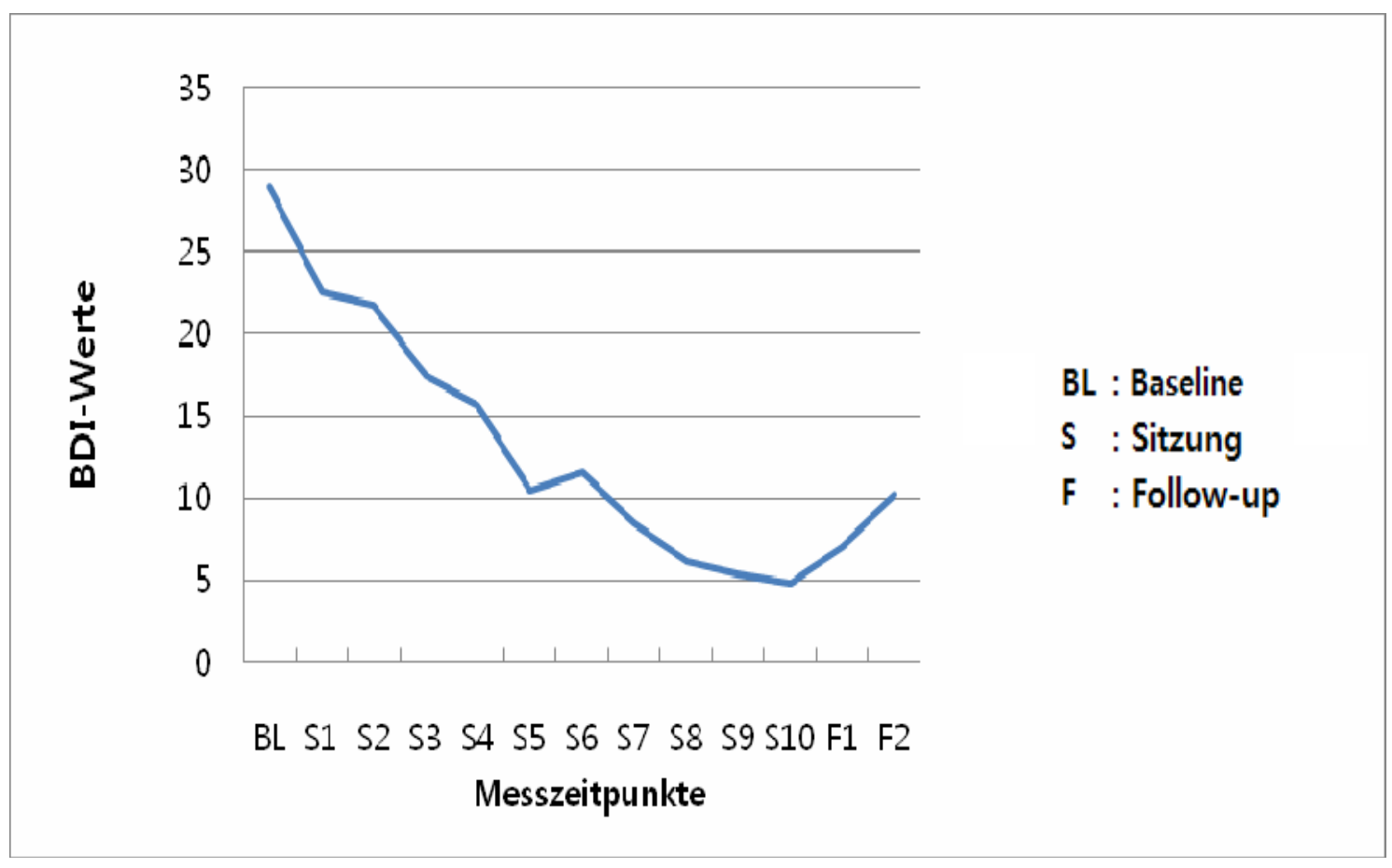

8.3.2 Das hilfreiche Erlebnis während des Programmsverlaufs - Bewertung durch die Teilnehmerinnen

Jede Teilnehmerin wählt nach jeder Sitzung ein Erlebnis aus und berichtet mittels des Instruments "IHE", was für sie in der vorangehenden Sitzung am hilfreichsten gewesen ist. Die Teilnehmerinnen werden aufgefordert, das hilfreichste Erlebnis in jeder Sitzung mit einem Satz zu benennen und den Prozess des Erlebnisses zu beschreiben. Die aufgeschriebenen Erlebnisse werden von drei Untersucherinnen analysiert und katagorisiert. Falls sie nicht übereinstimmen oder die Angaben uneindeutig sind, werden die Teilnehmerinnen dazu erneut befragt.

\subsubsection{Schritt 1: Die Sortierung der gesamten beschriebenen Erlebnisse}

Die Erlebnisse, die die Teilnehmerinnen nach jeder Sitzung ausgewählt haben, werden von drei Personen untersucht und sortiert (Tabelle 8.13). Die Erlebnisse sind nach insgesamt 24 Typen sortiert. 


\section{Ergebnisse}

Tab. 8.13: Die Typen des hilfreichsten Erlebnisses

\begin{tabular}{l}
\hline \multicolumn{1}{c}{ Erlebnisse } \\
\hline 01. emotionale Expression \\
02. Selbstöffnung \\
03. Spaß oder Erleichterung beim Ausdruck \\
04. Spaß bei der Exploration mit dem dargestellten Produkt \\
05. Das Gefühl zu haben, verstanden zu werden \\
06. Von anderen mich widerspiegeln zu lassen oder berührt zu werden \\
07. Interaktion mit der Therapeutin \\
08. Hilfe von anderen zu bekommen \\
09. Anderen Hilfe zu sein \\
10. Die Gruppe als eins zu erleben \\
11. Nähe zur Therapeutin und der Teilnehmerinnen zu empfinden \\
12. Alle haben ähnliche Probleme wie ich \\
13. Wahrnehmen der Ursache des Problems \\
14. Eigenes Problem mit verschiedenen Perspektiven zu sehen \\
15. Entwicklung oder Erkennen der Strategie \\
\hline 16. Ausprobieren der Strategie \\
17. Information zu bekommen \\
18. Besseres oder tieferes Selbstverständnis \\
19. Auftauchen früher blockierter Erlebnisse oder Erinnerungen \\
20. Einsicht zu gewinnen \\
\hline 1. Authentish ofer mit sin selbst
\end{tabular}

21. Authentisch oder mit sich selbst stimmig zu sein

22. Erkennen eigener Bedürfnisse

23. Selbstwertgefühl zu haben

24. Entdeckung eigener Ressourcen/Kräfte

\subsubsection{Schritt 2: Häufigkeit des hilfreichsten Erlebnisses in dem gesamten Prozess}

Die häufigsten Erlebnisse im gesamten Verlauf des Programms waren „emotionale Expression“ mit 28, dann "besseres und tiefes Selbstverständnis“ mit 27 Nennungen. Darauf folgen „Einsicht zu gewinnen“ mit 14, und "Selbstwertgefühl zu haben“ mit 11 Nennungen. An nächster Stelle kamen die Erlebnisse "Selbstöffnung“, „von anderen mich widerspiegeln zu lassen oder berührt zu werden“ und „die Gruppe als eins zu erleben“ mit jeweils 10 Nennungen. Das seltenste Erlebnis war „Information zu bekommen" mit einem einzigen Vorkommnis im gesamten Verlauf des Programms. 
Zur Veranschaulichung folgt eine Übersicht in Tabelleform (Tabelle 8.14). Die Erlebnisse, welche die Teilnehmerinnen in jeder Sitzung ausgesucht haben, werden auch dargestellt (siehe. Anhang).

Tab. 8.14: Häufigkeit des hilfreichsten Erlebnisses in dem gesamten Prozess

\begin{tabular}{|c|c|c|c|c|c|c|c|c|c|c|c|}
\hline \multirow{2}{*}{$\begin{array}{c}\text { Erlebnis } \\
\text { ID }\end{array}$} & \multicolumn{10}{|c|}{ Sitzung } & \multirow[t]{2}{*}{ Sum } \\
\hline & 1 & 2 & 3 & 4 & 5 & 6 & 7 & 8 & 9 & 10 & \\
\hline 01 & & & 2 & 1 & 5 & 4 & 5 & 5 & 3 & 3 & 28 \\
\hline 02 & 3 & 3 & 2 & 1 & 1 & & & & & & 10 \\
\hline 03 & 4 & 1 & 2 & 1 & & & & 1 & & & 9 \\
\hline 04 & 1 & 1 & & & 2 & 2 & & 1 & 2 & & 9 \\
\hline 05 & & 3 & 1 & 2 & & 1 & & & & & 7 \\
\hline 06 & 3 & 1 & 1 & 1 & 2 & 1 & 1 & & & & 10 \\
\hline 07 & 1 & 1 & 3 & 1 & 1 & & 1 & & & 1 & 9 \\
\hline 08 & & & & 1 & 1 & 1 & & 2 & & & 5 \\
\hline 09 & & & & & 1 & 1 & & 1 & 1 & & 4 \\
\hline 10 & & & & & & & 2 & 3 & 2 & 3 & 10 \\
\hline 11 & 1 & & & & 2 & 1 & 1 & & 1 & & 6 \\
\hline 12 & 1 & 3 & & & & & & & & & 4 \\
\hline 13 & & 2 & 3 & 2 & 1 & 1 & 1 & & & & 10 \\
\hline 14 & & & & 4 & 1 & 1 & 1 & & & & 7 \\
\hline 15 & & & & & & 1 & 4 & & 1 & & 6 \\
\hline 16 & & & & & & 1 & 1 & 3 & 2 & 1 & 8 \\
\hline 17 & & & & & & 1 & & & & & 1 \\
\hline 18 & 1 & 2 & 2 & 1 & 4 & 3 & 3 & 2 & 5 & 4 & 27 \\
\hline 19 & 1 & & 3 & 2 & & & & & & & 6 \\
\hline 20 & & & & & & & 1 & 4 & 5 & 4 & 14 \\
\hline 21 & 4 & 1 & & 1 & 1 & 1 & & & & & 8 \\
\hline 22 & 1 & 2 & 1 & 1 & & & & & & & 5 \\
\hline 23 & 1 & & 1 & 2 & & 1 & 1 & & & 5 & 11 \\
\hline 24 & & 2 & 1 & 1 & & 1 & & & & 1 & 6 \\
\hline Sum & 22 & 22 & 22 & 22 & 22 & 22 & 22 & 22 & 22 & 22 & 220 \\
\hline
\end{tabular}

Die Ergebnisse zeigen, dass es die hilfreichsten Erlebnisse nicht immer in jeder Sitzung gibt, sondern phasenabhängig. Das Programm wurde mit drei Phasen konzipiert und 


\section{Ergebnisse}

durchgeführt; nämlich "Aktive Exploration" als erste Phase in den ersten drei Sitzungen, "Aktiver Ausdruck“ als zweite Phase in der vierten bis achten Sitzung und „Integration“ als dritte Phase in der neunten und zehnten Sitzung. In der folgenden Tabelle wird die Häufigkeit der Erlebnisse den Phasen des Programms zugeordnet (Tabelle 8. 15).

Tab. 8.15: Häufigkeit der Erlebnisse in den drei Therapiephasen

\begin{tabular}{|c|c|c|c|}
\hline Erlebnisse & 1. Phase & 2. Phase & 3. Phase \\
\hline 01. emotionale Expression & 2 & 20 & 6 \\
\hline 02. Selbstöffnung & 8 & 2 & \\
\hline 03. Spaß oder Erleichterung beim Ausdruck & 7 & 2 & \\
\hline 04. Spaß bei der Exploration mit dem dargestellten Produkt & 2 & 5 & 2 \\
\hline 05. Das Gefühl zu haben, verstanden zu werden & 4 & 3 & \\
\hline $\begin{array}{l}\text { 06. Von anderen mich widerspiegeln zu lassen oder berührt zu } \\
\text { werden }\end{array}$ & 5 & 5 & \\
\hline 07. Interaktion mit der Therapeutin & 5 & 3 & 1 \\
\hline 08. Hilfe von anderen zu bekommen & & 5 & \\
\hline 09. Anderen Hilfe zu sein & & 3 & 1 \\
\hline 10. Die Gruppe als eins zu erleben & & 5 & 5 \\
\hline $\begin{array}{l}\text { 11. Nähe zur Therapeutin und der Teilnehmerinnen zu } \\
\text { empfinden }\end{array}$ & 1 & 4 & 1 \\
\hline 12. Alle haben ähnliche Probleme wie ich & 4 & & \\
\hline 13. Wahrnehmen der Ursache des Problems & 5 & 5 & \\
\hline 14. Eigenes Problem mit verschiedenen Perspektiven zu sehen & & 7 & \\
\hline 15. Entwicklung oder Erkennen der Strategie & & 5 & 1 \\
\hline 16. Ausprobieren der Strategie & & 5 & 3 \\
\hline 17. Information zu bekommen & & 1 & \\
\hline 18. Besseres oder tieferes Selbstverständnis & 5 & 13 & 9 \\
\hline 19.Auftauchen früher blockierter Erlebnisse oder Erinnerungen & 4 & 2 & \\
\hline 20. Einsicht zu gewinnen & & 5 & 9 \\
\hline 21. Authentisch oder mit sich selbst stimmig zu sein & 5 & 3 & \\
\hline 22. Erkennen eigener Bedürfnisse & 4 & 1 & \\
\hline 23. Selbstwertgefühl zu haben & 2 & 4 & 5 \\
\hline 24. Entdeckung eigener Ressourcen/Kräfte & 3 & 2 & 1 \\
\hline
\end{tabular}


8.3.2.3 Schritt 3: Die herausgefundenen phasenabhängigen bzw. -unabhängigen Hilfsfaktoren

Tab. 8.16: Die phasenabhängigen bzw. -unabhängigen Hilfsfaktoren

\begin{tabular}{|c|c|c|c|}
\hline \multirow[t]{2}{*}{ Hilfsfaktoren } & \multicolumn{3}{|c|}{ Häufigkeit in der Phase } \\
\hline & $\begin{array}{l}\text { Anfang } \\
\text { 1.-3.S }\end{array}$ & $\begin{array}{c}\text { Mitte } \\
\text { 4.-6./6.-8 }\end{array}$ & $\begin{array}{l}\text { Ende } \\
9 .-10 . S\end{array}$ \\
\hline \multicolumn{4}{|l|}{ die von der Anfangsphase abhängigen Hilfsfaktoren } \\
\hline - Selbstöffnung & 8 & $2 / 0$ & 0 \\
\hline - Spaß und Erleichterung beim Ausdruck & 7 & $2 / 0$ & 0 \\
\hline - Alle haben ähnliche Probleme wie ich & 4 & $0 / 0$ & 0 \\
\hline - Auftauchen früher blockierter Erlebnisse oder Erinnerungen & 4 & $2 / 0$ & 0 \\
\hline $\begin{array}{l}\text { - von anderen mich widerspiegeln zu lassen oder berührt zu } \\
\text { werden }\end{array}$ & 5 & $4 / 1$ & 0 \\
\hline - Wahrnehmen der Ursache des Problems & 5 & $4 / 1$ & 0 \\
\hline - Erkennen eigener Bedürfnisse & 4 & $1 / 0$ & 0 \\
\hline - Authentisch oder sich selbt stimmig zu sein & 5 & $3 / 0$ & 0 \\
\hline - Das Gefühl zu haben, verstanden zu werden & 4 & $3 / 0$ & 0 \\
\hline \multicolumn{4}{|l|}{ die von der Mittelphase anhängigen Hilfsfaktoren } \\
\hline - Hilfe von Anderen zu bekommen & 0 & 5 & 0 \\
\hline - Eigenes Problem mit verschiedenen Perspektiven zu sehen & 0 & 7 & 0 \\
\hline - Entwicklung oder Erkennen der Strategie & 0 & 5 & 1 \\
\hline - Anderen Hilfe zu sein & 0 & 3 & 1 \\
\hline $\begin{array}{l}\text { - Nähe zur Therapeutin und den Teilnehmerinnen zu } \\
\text { empfinden }\end{array}$ & 1 & 4 & 1 \\
\hline \multicolumn{4}{|l|}{ die von der Endphase abhängigen Hilfsfaktoren } \\
\hline - Die Gruppe als eins zu erleben & 0 & $0 / 5$ & 5 \\
\hline - Ausprobieren der Strategie & 0 & $0 / 3$ & 4 \\
\hline - Einsicht zu gewinnen & 0 & $0 / 5$ & 9 \\
\hline \multicolumn{4}{|l|}{ die phasenunabhängigen Hilfsfaktoren } \\
\hline - Emotionale Expression & 2 & 20 & 6 \\
\hline - Spaß bei der Exploration des dargestellten Produkts & 2 & 5 & 2 \\
\hline - Interaktion mit der Therapeutin & 5 & 4 & 1 \\
\hline - Besseres und tieferes Selbstverständnis & 5 & 13 & 9 \\
\hline - Selbstwertgefühl zu haben & 2 & 4 & 5 \\
\hline
\end{tabular}




\section{Ergebnisse}

Das Programm GPD-J besteht aus drei Arbeitsphasen: Die ersten drei Sitzungen dienen der „erlebnisorientierten Exploration“, die vierte bis achte Sitzung ist die Phase „zum aktiven Ausdruck“ und die neunte sowie zehnte Sitzung ist die Endphase „zur Integration" (siehe Kap. 4.2.2 , 6.2.4. und Anhang).

Einige Erlebnisse treten in der ersten Phase, jedoch nicht in der zweiten oder/und dritten Phase auf, und umgekehrt ebenso. Diese in bestimmten Phasen auftretenden Erlebnisse nenne ich "phasenabhängige“ bzw. "phasenunabhängige“ Hilfsfaktoren. Die in der vorliegenden Arbeit herausgefundenen phasenabhängigen Hilfsfaktoren sind dreierlei Art, nämlich die am Anfang, in der Mitte und am Ende der Therapie vorkommenden hlifreichsten Erlebnisse. Die Erlebnisse, die in der Therapie kontinuierlich als hilfreich aufgetreten sind, nenne ich „die phasenunabhängigen Hilfsfaktoren". Sie wurden in Tabelle 8. 16 aufgelistet.

\subsubsection{Schritt 4: Kategorisierung des hilfreichsten Erlebnisses}

Zur Kategorisierung werden „die 10 therapeutischen Faktorkategorie“ von Bloch et al. (1979), das „Session Impact System“ (Elliot 1985), das System des „Taxonomy of helpful impacts“ (Elliot \& Wexler 1994), „das multidimensionale System in Gruppen -Counseling" (Kivlighan et al. 1996) und das Kategoriensystem des "Good Moments" in der Gestalttherapie (Boulet et al. 1992) in Betracht gezogen.

Die hilfreichsten Erlebnisse werden sortiert und kategorisiert und in Tabelle 8.17 aufgelistet. Bei den Erlebnissen, die die Teilnehmerinnen in der Sitzung als am hilfreichsten bewerteten, werden vier verschiedene Foki aufgelistet: Der Fokus auf die Expression, der Fokus auf den Kontakt mit anderen, der Fokus auf das eigene Problem und der Fokus auf die Selbstwahrnehmung. 


\section{Ergebnisse}

Tab. 8.17: Kategorisierung der Erlebnisse für das hilfreiche Erlebnis

\begin{tabular}{lc}
\hline \multicolumn{1}{c}{ Erlebnisse } & Kategorie \\
\hline 11. emotionale Expression & 1. \\
12. Selbstöffnung & Fokus auf die \\
13. Spaß oder Erleichterung beim Ausdruck & Expression \\
14. Spaß bei der Exploration mit dem dargestellteten Produkt & \\
\hline
\end{tabular}

21. Das Gefühl zu haben, verstanden zu werden.

2.

22. Von anderen mich widerspiegeln $\mathrm{zu}$ lassen oder berührt $\mathrm{zu}$

Fokus auf den werden

Kontakt mit anderen

23. Interaktion mit der Therapeutin

24. Hilfe von anderen zu bekommen

25. Anderen Hilfe zu sein

26. Die Gruppe als eins zu erleben

27. Nähe zur Therapeutin und den Teilnehmerinnen zu empfinden

31. Alle haben ähnliche Probleme wie ich

3.

32. Wahrnehmen der Ursache des Problems

Fokus auf das Problem

33. Eigenes Problem mit verschiedenen Perspektiven zu sehen

34. Entwicklung oder Erkennen der Strategie

35. Ausprobieren der Strategie

36. Information zu bekommen

41. Besseres oder tiefes Selbstverständnis

4.

42.Auftauchen der früher blockierten Erlebnisse oder Fokus auf die Erinnerungen Selbstwahrnehmung

43. Einsicht zu gewinnen

44. Authentisch oder mit sich selbst stimmig zu sein

45. Erkennen eigener Bedürfnisse

46. Selbstwertgefühl zu haben

47. Entdeckung eigener Ressourcen/Kräfte 


\section{Ergebnisse}

Die Häufigkeiten der hilfreichen Erlebnisse sind einschliesslich der Kategorien der Tabelle $8.18 \mathrm{zu}$ entnehmen. Am häufigsten wird "Selbstwahnehmung", dann „Expression“, „Kontakt mit anderen“ und - am seltensten - „Problem“ genannt.

Tab. 8.18: Häufigkeit der hilfreichen Erlebnisse

\begin{tabular}{lccccccccccc}
\hline \multicolumn{1}{c}{ Kategorie } & \multicolumn{1}{c}{ Sitzung } & \multicolumn{1}{c}{ Sum } \\
\cline { 2 - 10 } & $\mathbf{1}$ & $\mathbf{2}$ & $\mathbf{3}$ & $\mathbf{4}$ & $\mathbf{5}$ & $\mathbf{6}$ & $\mathbf{7}$ & $\mathbf{8}$ & $\mathbf{9}$ & $\mathbf{1 0}$ & \\
\hline 1.Expression & $\mathbf{6}$ & 5 & 6 & 3 & $\mathbf{8}$ & 6 & 5 & 7 & 5 & 3 & $\mathbf{5 4}$ \\
\hline 2.Kontakt mit anderen & 5 & 5 & 5 & 5 & $\mathbf{7}$ & 5 & 5 & 6 & 4 & 4 & $\mathbf{5 1}$ \\
\hline 3.Problem & 2 & 5 & 3 & 7 & 2 & 5 & $\mathbf{7}$ & 3 & 3 & 0 & $\mathbf{3 7}$ \\
\hline 4.Selbstwahrnehmung & 9 & 7 & 8 & 8 & 5 & 6 & 5 & 6 & 10 & $\mathbf{1 4}$ & $\mathbf{7 8}$ \\
\hline
\end{tabular}

Anhand dieser Daten wird ein Verlauf der Häufigkeiten der hilfreichen Erlebnisse in Abbildung 8.10 dargestellt, um zu untersuchen, ob sich die Häufigkeit des hilfreichen Erlebnisses unterschiedlich in jeder Sitzung zeigt und, wenn ja, welche Kategorien am häufigsten auftreten.

Abb. 8.10: Entwicklung der hilfreichen Erlebnisse über alle Sitzungen

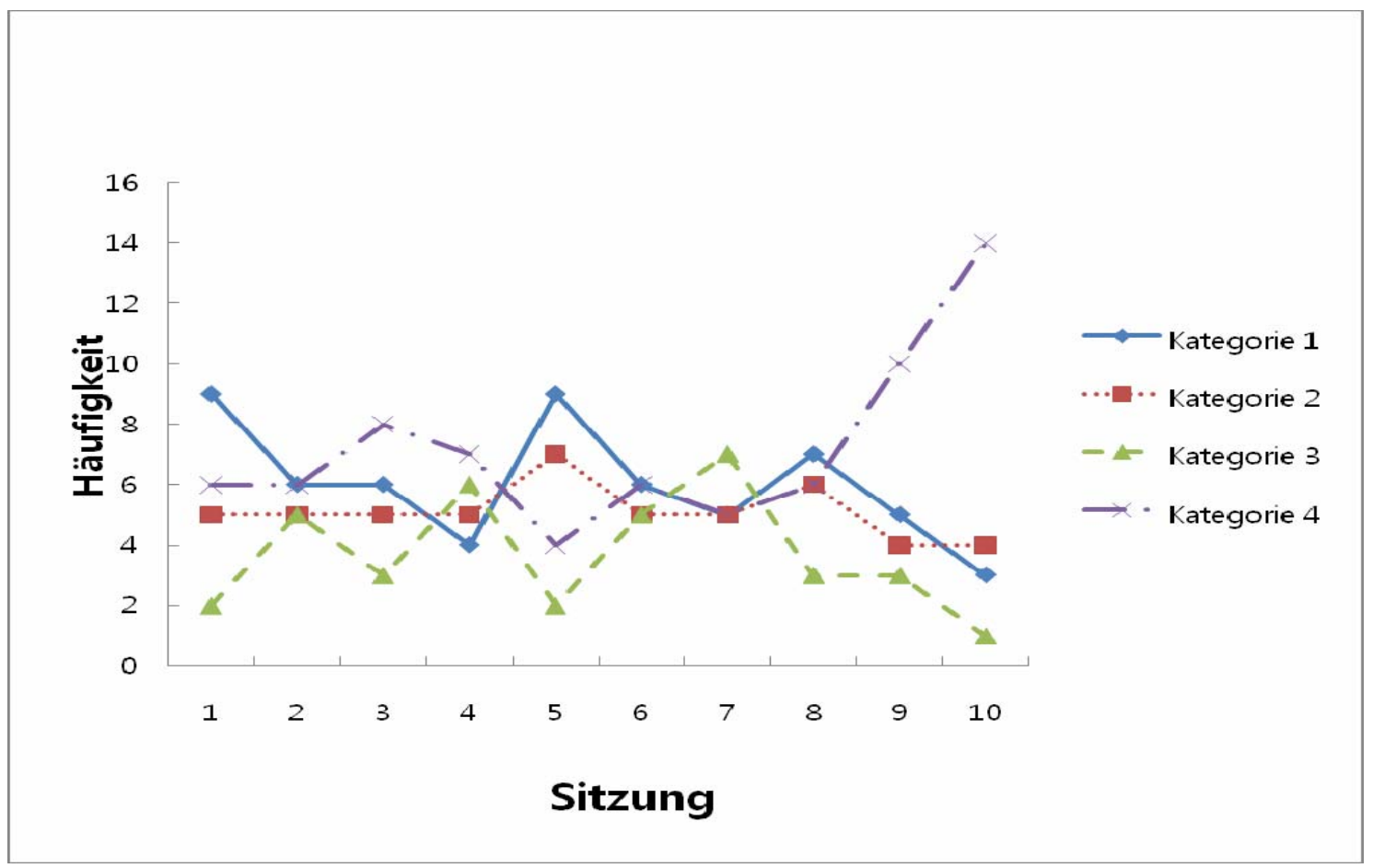

Die Kategorie 1, „Expression“, zeigt sich in der ersten bzw. fünften Sitzung am häufigsten im gesamten Verlauf des Programms. Die Kategorie 2, „Kontakt mit 


\section{Ergebnisse}

anderen“, zeigt sich nicht dramatisch von Anfang bis zum Ende des Verlauf des Programms, aber am häufigsten in der fünften Sitzung. Die dritte Kategorie, „Problem", ist am häufigsten in der siebten Sitzung während des Programmverlaufs. Die Kategorie 4, "Selbstwahrnehmung“, steigt ab der achten Sitzung bis zum Ende bemerkenswert stark an und zeigt sich am häufigsten in der zehnten Sitzung.

\subsection{Annahme des Programms : Rückmeldung der Teilnehmerinnen}

\subsubsection{Quantitative Angaben}

Die Teilnehmerinnen haben folgende Fragen beantwortet.

Frage 1. War das Programm für Sie hilfreich? Bewerten Sie bitte von 0 (überhaupt nicht) bis 10 (absolut).

Bei der Frage bewerteten die Teinehmerinnen das Programm durchschnittlich mit 9,4 Punkte als hilfreich. Sie bewerteten es mit 7 bis 10 Punkten.

Frage 2. Ist das Programm empfehlenswert für andere Personen mit Depressionen? Bewerten Sie bitte von 0 (überhaupt nicht) bis 10 (absolut).

Bei der Frage bewerteten sie das Programm durchschnittlich mit 9,6 Punkte bewertet. Die Bewertungen lagen zwischen 8 und 10 Punkten.

\subsubsection{Rückmeldung zum Programm}

Die Teilnehmerinnen wurden in einer offenen Frage aufgefordert, Rückmeldung darüber zu geben, ob sie während oder nach der Teilnahme an dem Programm irgendwelche Veränderung beobachtet haben. Für die Antwort haben die Teilnehmerinnen in der Sitzung für „Rückmeldung“ schriftlich abgegeben.

In den Rückmeldungen kristallisieren sich unterschiedliche Aspekte heraus. Eine vertiefte qualitative Auswertung dieser Rückmeldungen wäre sehr interessant, würde aber den Rahmen dieser Arbeit sprengen. Dennoch gaben die Rückmeldungen, ohne den Anspruch einer systematischen qualitativen Auswertung, einen Einblick in die Akzeptanz des Präventionsprogramms. Die Kommentare jeder Teilnehmerin sind in Tabelle 8.19 verkürzt dargestellt.

Obwohl die Teilnehmerinnen aufgefordert wurden, nicht nur positive sondern auch negative Veränderungen zu berichten, berichteten sie keine negativen Veränderungen.

Die Rückmeldungen bezogen sich auf Veränderungen bei den eigenen Gefühlen, auf das Verhalten in interpersonalen Beziehungen, auf den Erwerb einer Fähigkeit, z.B Entscheidung, Ausdruck, Verständnis für andere und Zuhören (Tabelle. 8.15). Für die 


\section{Ergebnisse}

Darstellung der Veränderungen in Tabelle 8.15 wurden die Veränderungen über Depression oder depressiven Zustand absichtlich entfernt. z.B. Ich bin nicht mehr depressiv oder ich fühle mich nicht mehr depremiert.

Tab. 8.19: Rückmeldungen über die Veränderungen während oder nach der Teilnahme an dem Programm

\begin{tabular}{|c|c|}
\hline ID & Rückmeldungen über die Veränderungen \\
\hline 101 & $\begin{array}{l}\text { Ich habe mehr Sicherheitsgefühl, denke positiver als vorher, bin stolzer auf } \\
\text { mich und habe bessere Beziehungen zu meiner Familie und meinen Freunden. }\end{array}$ \\
\hline 102 & $\begin{array}{l}\text { Ich fühle mich leichter, habe eine positivere Haltung zu mir und anderen, } \\
\text { drücke mich besser aus. Das macht mir Spaß. }\end{array}$ \\
\hline 103 & $\begin{array}{l}\text { Ich habe bessere Entscheidungsfähigkeit, habe weniger Problemme mit } \\
\text { meinen Freunden, bin nicht mehr schüchtern. Ich fühle mich klarer, da ich } \\
\text { durch die Teilnahme am Programm gelernt habe, mich zu fragen, wie ich mich } \\
\text { fühle. }\end{array}$ \\
\hline 104 & $\begin{array}{l}\text { Mein Charakter war zurückhaltend, ist jetzt aber nicht mehr, sondern aktiver. } \\
\text { Ich fühle mich erleichtert. Es ist weg, mich spontan über Wut auszudrücken, } \\
\text { was früher üblich war. }\end{array}$ \\
\hline 105 & $\begin{array}{l}\text { Ich lernte beim Programm, wie es möglich ist, mein Problem mit } \\
\text { verschiedenen Perspektiven anzuschauen, so dass ich mich nicht mehr fühlen } \\
\text { muss, als würde ich in eine Sackgasse fallen. Es ist jetzt schon gewöhnlich, } \\
\text { mich zu fragen, wie ich mich momentan fühle. }\end{array}$ \\
\hline 106 & $\begin{array}{l}\text { Bei der Teilnahme habe ich angefangen, Tagebuch zu schreiben, indem ich } \\
\text { mich nicht nur auf die Sache sondern auch über meine Gefühle konzentriere. } \\
\text { Ich fühle mich wertvoll und liebevoll. }\end{array}$ \\
\hline 107 & $\begin{array}{l}\text { Ich fühle mich selbständiger und erleichtert. Die Leute und die Welt sehen } \\
\text { freundlicher aus. Deshalb habe ich wenig Probleme mit meinen Freuden. }\end{array}$ \\
\hline 108 & $\begin{array}{l}\text { Ich bin mir sicherer, was ich will und wo ich bin. Ich drücke mich besser aus, } \\
\text { behaupte mich stärker und klarer. }\end{array}$ \\
\hline 109 & $\begin{array}{l}\text { Ich denke positiver, habe weniger Wut und fühle mich selbständiger und } \\
\text { aktiver. }\end{array}$ \\
\hline 110 & $\begin{array}{l}\text { Ich denke posotiver und fühle mich als ein wertvolles Wesen. Ich drücke mich } \\
\text { besser und offener aus. Dafür frage ich immer nach meinem Gefühl. }\end{array}$ \\
\hline 111 & $\begin{array}{l}\text { Ich war ein introvertierter Typ, bin das aber nicht mehr. Ich fühle mich } \\
\text { selbständiger und optimistischer. Ich habe eine positive Haltung zu meinem } \\
\text { Vater, mit dem ich früher immer Probleme hatte. }\end{array}$ \\
\hline 112 & Ich habe die Fähigkeit, nicht nur mich sondern auch andere zu verstehen. Ich \\
\hline
\end{tabular}




\section{Ergebnisse}

bin stolzer auf mich, selbständiger, fühle mich wertvoll und denke positiver.

201 Ich fühle mich erleichtert, frei und selbständiger. Mein Ausdruck ist klarer geworden. Ich denke über die Dinge positiver.

202 Es kommt kaum noch vor, dass ich plötzlich Wut zeige. Mein Blick auf mich und andere ist positiver und freundlicher. In interpersonaler Beziehung bin ich aktiver und denke positiver.

203 Meine Gedanken werden positiver, besonders die Gedanken über mich. Ich drücke mein negatives Gefühl mehr aus, wenn ich mich inwohlfühle.

204 Ich habe keine Langeweile in meinem Leben. Es sieht alles liebevoll aus. Ich treffe mehr Leute, verhalte mich freundlicher zu anderen und ehrlicher.

205 Dankbarkeit und Ernst, was ich früher nicht hatte. Ich bin positiver und leichter. Meine Familie, meine Freunde und die Leute, die um mich stehen, empfinde ich als wichtig.

206 Ich fühle mich selbständiger, nicht mehr zurückhaltend und konzentriere mich ganz gut auf alles, was ich will. Mit meiner Familie habe ich bessere Beziehung.

207 Ich fühle mich nicht mehr allein, die Welt ist freundlicher und ich bin aktiver in interpersonaler Beziehung.

208 Ich habe seltener Wut. Ich verstehe mich besser mit meinen Freunden, da ich ihnen mehr vertraue. Die Fähigkeit, zuzuhören, und mich anderen zuzuwenden, lernte ich durch die Teilnahme.

209 Ich denke positiver und lebendiger. Ich spreche erst meinen Vater an, was ich mir früher nicht vorstellen konnte.

210 Ich entscheide mich besser und schneller, habe eine bessere Beziehung mit meiner Schwester. Ich bin ehrlicher gegenüber anderen. 


\section{Diskussion}

\section{Diskussion}

Wie bereits beschrieben waren die Ziele dieser Studie die Entwicklung eines Präventionsprogramms bei Depressionen und die Überprüfung seiner Wirksamkeit. Darüber hinaus war zu untersuchen, was während des Verlaufs des Programms bei den Teilnehmern als hilfreich aufgenommen wurde. Die Stichprobe bestand aus weiblichen Jugendlichen mit subklinisch depressiven Symptomen im Alter von 16 bis 18 Jahren an einem Gymnasium in Korea. Das Untersuchungsdesign war die Bildung experimenteller Zwillige, d.h. einer Versuchs- und Kontrollgruppe, die ein homogenes Bild haben und durch zufällige Auswahl gebildet wurden. Die beiden Gruppen waren in allen untersuchten Aspekten zueinander homogen. An drei Zeitpunkten wurden psychologische Tests durchgeführt: Prä (vor der Intervention), Post (direkt anschließend an die Interventionsphase), und zwei Follow-ups (drei bzw. sechs Monate nach der Intervention).

Die Diskussion wird sich zunächst damit beschäftigen, inwiefern Ziele der Untersuchung gelungen sind, und wird auf die Fragen der Wirksamkeit des Programms und des Prozesses des Programms im Hinblick auf hilfreiche Erlebnisse für die Teilnehmerinnen eingehen. Die Ergebnisse zur Wirksamkeit des Programms werden in Bezug auf die Hypothesen diskutiert und die Ergebnisse bzgl. der hilfreichen Erlebnisse im Hinblick auf die Mechanismen der Programmwirksamkeit diskutiert. Außerdem führt die Diskussion zu einem Ausblick auf die Relevanz sowohl der Prävention von Depression bei weiblichen Jugendlichen, aus denen die Stichprobe in dieser Studie bestand, als auch auf die Entwicklung der verschiedenen Programme und ihre Angebote im Schulkontext im Rahmen einer Maßnahme der Gesundheitsförderung.

Die Einschränkungen und Schwächen dieser Untersuchung und Vorschläge für weitere Forschung werden in der Diskussion nicht ausgelassen.

\subsection{Wirksamkeit des Programms}

\subsubsection{Effekte direkt nach Beendigung des Programms}

Es zeigten sich bereits direkt nach Beendigung des Programms statistisch signifikante Unterschiede zwischen der Präventionsgruppe und der Kontrollgruppe, obwohl die beiden Gruppen vor dem Programm homogen waren. 


\section{Diskussion}

Die untersuchten Hypothesen,

- dass die Gruppe der Probanden des Präventionsprogramms nach der Intervention im Vergleich zur Kontrollgruppe signifikant größere Fortschritte bei der Reduzierung der Kernsymptome machen wird,

- dass die Gruppe der Probanden des Präventionsprogramms sich nach der Intervention im Vergleich zur Kotrollgruppe sich bei den psychischen Beschwerden signifikant positiv verändern kann, und

- dass bei der Gruppe der Probanden des Präventionsprogramms nach der Intervention im Vergleich zur Kotrollgruppe sich beim psychischen Wohlbefinden signifikante positive Veränderungen zu verzeichnen ist,

können beibehalten werden, weil sich

- ein Kernsymptom nach der Intervention signifikant bei der Versuchsgruppe im Vergleich zur Kontrollgruppe verbesserte,

- bei einem Faktor des Tests für die psychischen Beschwerden signifikante positive Veränderungen der Versuchsgruppe gegenüber der Kontrollgruppe nach der Intervention gemessen wurden, und

- bei einem Faktor des Tests für das psychische Wohlbefinden signifikante positive Veränderungen der Versuchsgruppe gegenüber der Kontrollgruppe nach der Intervention gemessen wurden.

Die Varianzsnalyse mit Messwiederholung ergibt einen Haupteffekt der Bedingung „Versuch vs. Kontrolle“ in der Variablen „depressive Symptome“. Bei „depressiven Symptomen“ (BDI) sowie „Depressivität“ (SCL-90-R) zeigt die Präventionsgruppe eine starke Verbesserung, während die Kontrollgruppe eine leichte Verschlechterung zeigt. Diskussionseinleitend wird Bezug genommen auf die Konstellation der untersuchten Gruppen. Alle Teilnehmerinnen an dieser Studie haben sich selbst mit hoher Motivation für das Programm angemeldet. Nach der Diagnostik hatten sie die Chance, einen von zwei Terminen zu wählen und wurden danach in zwei Gruppen, also Versuchs- oder Kontrollgruppe, zufällig eingeteilt. In diesem Prozess wäre es möglich gewesen, dass die Teilnehmerinnen der Versuchsgruppe die Hoffnung und/oder den Placeboeffekt zeigten, und dass die Teilnehmerinnen der Kontrollgruppe umgekehrt eher enttäuscht wären. Obwohl sie bei der Information schon mit dieser Möglichkeit, nicht alle an dem Programm teilnehmen zu können, einverstanden waren, könnte man argumentieren, dass diese Reaktion bei der Kontrollgruppe ethisch abzuwägen ist. Solche ethischen Probleme sollten nicht verschwiegen werden, aber in diesem 


\section{Diskussion}

Dilemma befindet sich die empirische Therapieforschung immer, insbesondere bei der Überprüfung der Wirksamkeit von Therapieverfahren und im Kontext einer Forschung, die nicht rein praxisbezogen ist.

Die größten Mittelwertdifferenzen bei den psychischen Beschwerden wurden in der Präventionsgruppe auf der Skala "Depressivität” (Prä $M=3,004 ;$ Post $M=, 279 ; t=$ 48,396, $p<, 001)$ erreicht. Die signifikanten Mittelwerte wurden für die Skalen "Somatisierung" (Prä $M=2,076$; Post $M=$,526; $t=17,303, p<, 001$ ), "Interpersonale Unsicherheit" (Prä $M=2,287 ;$ Post $M=, 686 ; t=7,370, p<, 001$ ), „Feindseligkeit“ (Prä $M$ = 1,341; Post $M=, 397 ; t=5,789, p<, 001$ ), "Zwanghaftigkeit" (Prä $M=1,414 ;$ Post $M$ $=, 323 ; t=5,765, p<, 001$ ) und "Ängstlichkeit" (Prä $M=1,636$; Post $M=, 900 ; t=4,213$, $p<, 001)$ in der Präventionsgruppe ermittelt. Die Skalenmittelwerte der Abschlusserhebung haben sich deutlich denen der gesunden Normalstichproben angenähert oder fallen sogar niedriger aus, während die Mittelwertdifferenzen in der Kontrollgruppe nicht signifikant sind. Die Mittelwerte auf den Subskalen „Phobische Angst", "Paranoides Denken“ und „Psychotizismus" zeigen keine signifikanten Veränderungen in beiden Gruppen.

Kopka und seine Mitarbeiter (1994) untersuchten mit Hilfe der SCL-90-R(N=685), nach wievielen Therapiesitzungen sich welche Symptome wieder normaliserten, und fanden heraus, dass Veränderungen auf den Skalen „Depressivität", "Angst" und "Zwanghaftigkeit" sich sehr schnell vollziehen, während sich der Erfolg auf den anderen Skalen sehr langsam oder gar nicht einstellt. Dagegen würden Patienten mit charakterologischen Störungen erst späte oder keinerlei symptomatische Verbesserung zeigen (Steffan, 2002). In der vorliegenden Untersuchung wurde herausgefunden, dass Veränderungen auf den Skalen „Depressivität“, „Somatisierung“, „Interpersonale Unsicherheit", „Feindsleigkeit", „Zwanghaftigkeit“ und „Ängstlichkeit“ sich schnell und signifikant vollziehen, während die Mittelwerte auf den Subskalen „Phobische Angst", "Paranoides Denken" und "Psychotizismus" keine signifikanten Veränderungen in beiden Gruppen zeigten. Es kann eingeschätzt werden, dass sich das Alter der Klienten und die klinische Störung als Unterschiedfaktoren ergeben, da die 16- bis 18 jährigen Teilnehmerinnen in einem Gymnasium, das erst nach einer Eintrittsprüfung besucht werden darf, zur Schule gingen. D.h. sie sind vermutlich intelligenter als gleichaltrige Mitschüler.

In dieser Studie wurde das „psychische Wohlbefinden“ zur Untersuchung der subjektiven Lebensqualität einschließlich der Wirksamkeit des Programms eingesetzt. Die Ergebnisse der Studie (Diener \& Diener 1995; Kim et al. 2001; Ryff 1989; Ryff \& Keyes 1995;) legen deutlich nahe, dass die sechs Faktoren aus dem psychischen 


\section{Diskussion}

Wohlbefinden (PWB) jeweils ein unabhängiger Bestandteil sind, z. B. hat „Selbstakzeptanz" allein 37\% der Aussagekraft für das subjektive Wohlbefinden. Auch kann man davon ausgehen, dass sich die sechs Faktoren bei unterschiedlichen Altersgruppen, Kulturen und Geschlechtern unterschiedlich auf das psychische Wohlbefinden auswirken.

Die größten Mittelwertdifferenzen beim psychischen Wohlbefinden wurden in der Präventionsgruppe auf der Skala „Lebenssinn“ (Prä $M=1,773$; Post $M=3,783$; $t=$ $37,814, p<, 001)$ ermittelt. Darüber hinaus wurden bei allen Skalen signifikante Mittelwertdifferenzen festgestellt: "Autonomie“ (Prä $M=1,635$; Post $M=3,528 ; t=$ 34,163, $p<$,001), „Persönliches Wachstum“ (Prä $M=1,903$; Post $M=3,903 ; t=-33,723, p$ $<$,001), "Selbstakzeptanz" (Prä $M=1,596$; Post $M=3,983 ; t=-32,060, p<, 001$ ), „Beherrschung der Welt" (Prä $M=1,761$; Post $M=3,471 ; t=-24,441, p<, 001)$ und "Interpersonale Unsicherheit“ (Prä $M=1,668$; Post $M=3,948 ; t=-20,606, p<, 001$ ). Die Mittelwertdifferenzen der Kontrollgruppe waren hingegen nicht signifikant.

Diese Ergebnisse sind vielversprechend, um das zum psychische Wohlbefinden von Jugendlichen zu verstehen. Die Mittelwerte der Skalen „Beherrschung der Welt“ und "Interpersonale Unsicherheit" sind besonders niedrig im Vergleich zu den anderen Skalen, obwohl auch sie signifikant positive Veränderungen gezeigt haben. Als Grund ist anzunehmen, dass die Jugendlichen von ihrem Lebensumfeld und interpersonalen Beziehungen stark beeinflusst werden. Die Skala "Autonomie“ steht bei den Veränderungen an erster Stelle und die Skala „Interpersonale Unsicherheit“ steht hier an letzter Stelle. Diese Ergebnisse zeigen den Zustand der Jugendlichen ganz deutlich. Die wichtigsten Themen unter den Jugendlichen sind "Autonomie und Abhängigkeit“ sowie „Distanz und Nähe“. Man kann aus diesen Ergebnisse folgern, dass das Programm zur Verbesserung der „Autonomie“ bei Jugendlichen beiträgt und dass sich der Faktor „Autonomie“ auf die Linderung depressiver Symptome auswirkt.

\subsubsection{Langfristige Effekte nach drei und sechs Monaten}

Auch hier erweist sich der nachhaltige Effekt des Präventionsprogramms im Vergleich zur Kontrollgruppe hypothesenkonform und als statistisch durchgehend signifikant, wenn man die Veränderungsmaße aller abhängigen Variablen zusammenfasst.

Die untersuchten Hypothesen,

- dass die Versuchsgruppe im Vergleich zur Kontrollgruppe signifikant größere Fortschritte bei der Reduzierung der Kernsymptome zum Zeitpunkt 


\section{Diskussion}

nach drei bzw. sechs Monaten nach der Intervention machen wird,

- dass die Versuchsgruppe imVergleich zur Kontrollgruppe signifikant positivere Veränderungen bei den psychischen Beschwerden zum Zeitpunkt nach drei bzw. sechs Monaten nach der Intervention erreicht, und

- dass die Versuchsgruppe im Vergleich zur Kontrollgruppe signifikant positivere Veränderungen beim psychischen Wohlbefindenn zum Zeitpunkt nach drei bzw. sechs Monate nach der Intervention erreicht,

können beibehalten werden, weil sich

- ein Kernsymptom signifikant bei der Versuchsgruppe im Vergleich zur Kontrollgruppe im Zeitpunkt nach drei bzw. sechs Monaten nach der Intervention verbesserte,

- bei einem Faktor des Tests für die psychischen Beschwerden signifikante positive Veränderungen der Versuchsgruppe gegenüber der Kontrollgruppe im Zeitpunkt nach drei bzw. sechs Monaten nach der Intervention gemessen wurden, und

- bei einnem Faktor des Tests für das psychische Wohlbefinden signifikante positive Veränderungen der Versuchsgruppe gegenüber der Kontrollgruppe im Zeitpunkt nach drei bzw. sechs Monaten nach der Intervention gemessen wurden.

Insgesamt könnten die durchgehenden systematischen Gruppenunterschiede zwischen Präventions- und Kontrollgruppe bei der Varianzanalyse mit Messwiederholung mit allen Variablen unter den Bedingungen "Zeit“ und "Zeit x Gruppe (GPD-J vs. Kontrolle)“ als eindeutiger Hinweis auf die erwartete Wirksamkeit des Programms gewertet werden.

Die Varianzanalysen mit Messwiederholung der Variablen „BDI" und „Depressivität“ zeigen einen signifikanten Haupteffekt von „Zeit“ und weiterhin einen signifikanten Effekt von "Zeit x Gruppe“. Follow-up-Tests zeigen, dass dieser Effekt auf Unterschiede zwischen den Messpunkten in der Präventionsbedingung GPD-J zurückzuführen ist, während die Unterschiede in der Kontrollgruppe nicht signifikant sind. Es ist zu vermuten, dass der Effekt der Unterschiede zwischen den Messpunkten in der Präventionsgruppe nach 6 Monaten darauf zurückzuführen ist, dass sich ein Drittel der Teilnehmerinnen der Gruppe vor dem Anfang des Semesters in der Vorbereitung zur Prüfung (entspricht dem Abitur in Deutschland) befand.

In der Studie von Clarke et al.(1995) wurden die Jugendliche (durchschnittlich 15,3 Jahre alt, $70 \%$ Mädchen) mit erhöhten depressiven Gesamtscores, aber keiner 


\section{Diskussion}

affektiven Störung in einer indikativen Präventionsgruppe behandelt. In dieser Präventionsgruppe wurden 76 Probanden in 15 Sitzungen je 45 Minuten kognitivtherapeutisch behandelt und mit 74 Probanden einer Kontrollgruppe verglichen. Die Ergebnisse dieser Studie zeigen, dass die Interventionsffekte in Bezug auf die Skala „Depression“ nach 6 Monaten verschwunden waren, und dass neue affektive Störungen auch nach 12 Monaten in der Interventionsgruppe im Vergleich zur Kontrollgruppe weniger waren. In der Studie (Jaycox et al. 1994; Gillham et al. 1995; Gillham \& Reivich 1999; Zubernis et al. 1999) hatten Kinder (durchschnittlich 11,4 Jahre alt) mit erhöhten depresiven Gesamtscores an den Interventionsgruppen (69 Kinder) oder den Kontrollgruppen (74 Kinder) teilgenommen. Die indikativen Präventionsinterventionen bestanden aus 12 Sitzungen je 90 Minuten. Die Messzeitpunkte der zwei Skalen in Bezug auf Depression waren Prä- und Post-Test, sowie Follow-ups nach 6, 12, 18, 24, 30 und 36 Monaten. Bei allen Follow-ups berichteten in der Interventionsgruppe weniger Kinder "moderat depressive“ Symptome als in der Kontrollgruppe (zit. nach Wiesner \& Reitzle, 2001).

Vergleicht man die langfristigen Effekte der indikativen Präventionsinterventionen mit anderen Studien, ist es erforderlich, weitere Follow-ups, z.B. nach 12 Monaten nach dem Programm bei der Präventionsintervention GPD-J durchzuführen.

Das Betrachten der Varianzanalysen mit Messwiederholung unter Einbeziehung der beiden Messzeitpunkte nach drei und sechs Monaten lässt feststellen, dass erwartungsgemäß signifikante Veränderungen auf die Variablen „allgemeine psychische Beschwerden" und "psychisches Wohlbefinden" nachgewiesen wurden. Follow-up-Tests zeigen, dass dieser Effekt auf Unterschiede zwischen den Messpunkten in der Präventionsbedingung GPD-J zurückzuführen ist, während die Unterschiede in der Kontrollgruppe nicht signifikant sind.

Im Vergleich der Mittelwerte aller Skalen SCL mit Normierungsstichproben zeigt die Versuchsgruppe den größten Abstand bei der Skala „Somatisierung“ ( $M(M G)=0,77$, nach 3 Monaten $M=0,264$, nach 6 Monaten $M=0,374$ ), während sich eine geringere Mittelwertdifferenz bei der Skala "Unsicherheit" $(M(M G)=1,23$, nach 3 Monaten = 1,144, nach 6 Monaten = 1,122) zeigt. Die Mittelwerte der Skala „Ängstlichkeit“ bei der Versuchsgruppe nach 3 bzw. nach 6 Monaten sind höher als bei den Normierungsstichproben. Bei der Kontrollgruppe sind die Mittelwerte fast aller Skalen höher als bei Normierungsstichproben im Vergleich prä, 3 bzw. 6 Monate. Die Mittelwerte der Skalen "Paranoides Denken“ und "Psychotizismus" in beiden Gruppen sind niedriger als bei den Normierungsstichproben zu allen Messzeitpunkten. 3 Monate nach dem Ende des Programms senken sich die Mittelwerte bei der 


\section{Diskussion}

Versuchsgruppe bei allen PWB-Subskalen, und ebenso nach weiteren 3 Monaten, mit Ausnahme der Skala „Lebenssinn“. Die Mittelwerte der Skala „Beherrschung der Welt" ändern sich bei der Versuchsgruppe am geringsten im Vergleich mit den Veränderungen anderen Skalen. Der Mittelwert der Skala „Lebenssinn“ senkt sich in beiden Gruppen gleich 3 Monate nach dem Ende des Programms. Besonders diskussionsleitend ist, dass die Skalen „interpersonale Unsicherheit“ (SCL-90-R) und „Beherrschung der Welt" (PWB) nach 6 Monaten im Vergleich zum Prä-Test die gerinsten Veränderungen hatten. Obwohl die Skala "Autonomie" (PWB) die größten Veränderungen nach dem Programm zeigte, ging „Beherrschung der Welt“ nach 6 Monaten zurück auf die Werte wie vor dem Programm. Der Grund, wie oben schon erwähnt, könnte sein, dass die Jugendlichen trotz ihres Wunschs nach Unabhänglichkeit von draußen, z.B. durch interpersonale Beziehung und im Lebenskontext empfindlicher beeinflusst werden könnten als andere, oder dass der Effekt des Präventionsprogramms GPD-J die beiden Variablen nicht nachhaltig zu beeinflussen vermag. Leben die Jugendlichen am Gymnasium in koreanischer Gesellschaft, die starke Konkkurenz für den Eintritt in eine gute Universität erzwingt, ist es schwierig, den Alltag und die äußerliche Gegebenheit zu organisieren, was nach Ryff (1989) der Beherrschung der Lebenswelt entspricht.

Aber es ist doch bemerkenswert, dass die Teilnehmerinnen am Programm GPD-J nach dem Programm zwei Selbsthilfegruppen entwickelt haben. Sie treffen sich oft nach dem Programm in der Schule, planen einen Club für „Peer-Counseling“ in der Schule zu entwickeln und melden sich seit dem Programm oft Online bei der Therapeutin, wenn sie Hilfe brauchen. D.h. dass auch langfristig das Verhalten beeinflusst wurde. Aber dies wurde in der vorliegenden Studie nicht weiter untersucht.

\subsection{Prozessanalyse}

\subsubsection{Zusammenhang der Verlaufsentwicklung von Depression und Hilfsfaktoren}

Zur Prozessanalyse wurde die Verlaufsentwicklung des BDI-Wertes untersucht. Um Depressionsverläufe während des Programms zu untersuchen, wurden die Werte von Becks Depressionsinventar nach jeder Sitzung von den Teilnehmerinnen erhoben. Aus Lee und Songs Studie (1991) ergab sich, dass man bei weiblichen Jugendstichproben bei Werten zwischen 16 und 23 von subliminaler Depression spricht, während Werte ab 24 auf eine klinisch manifeste Depression hinweisen.

Die Anzahl der BDI-Werte unter 16 erhöhte sich nach der fünften Sitzung deutlich, und 
die Anzahl der Werte über 24 sank schon nach der ersten Sitzung. Ab der neunten Sitzung zeigen alle Teilnehmerinnen BDI-Werte von unter 16.

Wie sich in der Abbildung 9 zeigt, hat eine dramatische Veränderung der BDI-Werte von der vierten zur fünften Sitzung an stattgefunden. In der vierten Sitzung waren die BDI-Werte 15,727 (SD = 5,938), und dann 10,500 (SD = 3,335) in der fünften Sitzung. Der geringste Unterschied bestand zwischen den BDI-Werten der zehnten Sitzung $(\mathrm{M}=$ $4,818, \mathrm{SD}=6,727)$ und der neunten Sitzung ( $M=5,364, \mathrm{SD}=1,840)$. Dann erhöhen sich die BDI-Werte nach drei Monaten $(\mathrm{M}=7,000, \mathrm{SD}=7,810)$ und nach sechs Monaten $(\mathrm{M}=$ $10,272, \mathrm{SD}=4,113$ ) sowie nach Endes des Programms.

Es ist merkwürdig, dass sich erstens die BDI-Werte so schnell positiv geändert haben und dass sie zweitens nach der ersten bzw. fünften Sitzung den größten Abstand, bezogen auf den vorherigen Messzeitpunkt, hatten. Bei der ersten Sitzung ist zu vermuten, dass die Teilnehmerinnen den Placeboeffekt mit Erleichterungsgefühlen hatten, da sie für die Teilnahme am Program GPD-J angenommen wurden.

In der fünften Sitzung haben sich die Teilnehmerinnen mit dem Thema "Ausdruck negativer Gefühle zum Selbst“ beschäftigt. In dieser Sitzung war der häufigste Hilfsfaktor die „emotionale Expression“. Die Ziele waren in dieser Sitzung: Aktiver Ausdruck der negativen Gefühle zum Selbst mit den Medien, Auflösung der Selbstkonflikte im bestimmten Lebenskontext (Schule oder Familie) und Förderung der Selbstakzeptanz, Selbstregulation und Selbstunterstützung. Die Teilnehmerinnen haben mit den Bildern, die in der vierten Sitzung von ihnen selbst gemaltt wurden (siehe Anhang), gearbeitet. Wenn sich die negativen Gefühle von Teilnehmerinnen auf einen bestimmten Lebenskontext bezogen wurden ggf. die Bilder, die in der zweiten und dritten Sitzung gemalt worden waren, eingesetzt. Wenn das Gefühl z.B. in der Beziehung mit Schulkameraden verursacht wurde, arbeitete die Betroffene mit dem Bild, das in der dritten Sitzung erstellt worden war. Die Teilnehmerinnen, die zu Einzelarbeit mit der Theraputin in dem Gruppensetting bereit waren, haben weiter gearbeitet. Zwei Teilnehmerinnen wollten nicht Einzelarbeit in der Gruppe machen. Aber sie haben in der achten Sitzung mit dem Rollenspiel gearbeitet. Daraus lässt sich folgern, dass die fünfte Sitzung mit dem Thema "Aktiver Ausdruck negativer Gefühle zum Selbst“ unter Einbeziehung des Hilfsfaktors „Emotionale Expression“ die positivste Veränderung bei den BDI-Werten moderiert hat. 


\section{Diskussion}

\subsubsection{Zusammenhang zwischen den Arbeitsprinzipien und dem hilfreichsten Erlebnis}

Ergebnisforschung sollte mit intensiver Prozessanalyse verbunden werden. Bei der Durchführung werden sehr unterschiedliche Dinge ermittelt. Prozessforschung muss so intensiv betrieben werden, dass ein Forschungsbericht den Rezipienten über drei Fragen informiert: Wie verlief der Veränderungsprozess, welche Veränderungsschritte entstanden $\mathrm{zu}$ welchem Zeitpunkt und durch welche Faktoren oder Beziehungskonstellationen wurden sie bewirkt bzw. erleichtert? (Fischer et al., 1998) Deshalb wurde die Prozessanalyse mit dem hilfreichsten Erlebnis dargestellt, damit die Studie der Forderung nach systemischer Nachvollziehbarkeit durch Außenstehende und Rekonstruierbarkeit der Veränderungschritte Rechnung gerecht werden kann.

Im Kapitel. 4.2.2. wurden die Prozess- und themenbezogenen Interventionsprinzipien des Programms in drei Schritten konzipiert, wobei jeder Schritt ein genaues Ziel erreicht. Ob das Programm mit den Prinzipien die vorgestellten Ziele erreicht hat, wurde in der Untersuchung der Prozessanalyse mit dem hilfreichsten Erlebnis überprüft.

In den ersten drei Therapiesitzungen ging es um Problemaktualisierung und Ressourcenaktivierung durch erlebnisorientierte Exploration. In dieser Phase wurden nicht nur Probleme sondern auch Ressourcen in den drei Ebenen "Selbst", „Lebenskontext Schule sowie Familie“ durch kreative Medien erarbeitet, damit die Teilnehmerinnen ihre Probleme in Verbindung mit ihrem Lebensfeld wahrnehmen und aktualiseren und darüber hinaus ihre Ressourcen und Resilienzen sowie ihre Problemaktualiserung entdecken und aktivieren.

Die Ergebnisse der Prozessanalyse mit dem hilfreichsten Erlebnis zeugen davon, dass das Ziel in dieser Phase erreicht wurde. In der ersten Sitzung war das Erlebnis „emotionale Expression“ gar nicht von Teilnehmerinnen als das hilfreichste ausgewählt, sondern „Selbstöffnung“ (5 Teilnehmer), „Spaß oder Erleichterung beim Ausdruck" (4 Personen). Das heißt, dass das Programm in der ersten Sitzung die Interessen von Teilnehmern erweckt hat und sie motiviert hat, weiter $\mathrm{zu}$ arbeiten. Tatsächlich haben viele Teilnehmer nach dieser Sitzung beschrieben, wie die Arbeit mit kreativen Medien sie neugierig machte. Diese Ergebnisse sollten zur Konzipierung eines Programms der Intervention bei Jugendlichen betrachtet werden, da gerade bei der Therapie und Beratung für Jugendliche die Drop-Out-Quote sehr hoch ist.

In der zweiten Sitzung waren die am häufigsten genannten Erlebnisse „Selbstöffnung“, „Das Gefühl zu haben, verstanden zu werden“ und „Alle haben ähnliche Probleme wie 


\section{Diskussion}

ich", die jeweils von drei Personen ausgewählt wurden. Die Erlebnisse "Selbstöffnung“ und „Alle haben ähnliche Probleme wie ich“ wurde in den letzten vier Sitzungen gar nicht genannt. d.h. die drei Erlebnisse trugen bei den Teilnehmerinnen zum Entstehen von Sicherheit und Geborgenheit in der Gruppe bei. "Selbstöffnung" und "Verstanden-zu-werden“ wirken zusammen auf die Gruppenarbeit ein. Es ist besonders wichtig anzumerken, dass sich das Erlebnis „Alle haben ähnliche Probleme wie ich" auf das Gefühl der Befreiung bezieht, sodass die Teilnehmer sich sicher und erleichtert fühlen. Es ist auch denkbar, dass nicht nur die depressiven sondern alle Jugendlichen imstande sind, sich mit dem Thema „normal oder unnormal“ zu beschäftigen. Sie sorgen sich um die Veränderungen von Körper, Emotion, Beziehung usw. und fragen sich, ob es normal ist und wie es bei anderen sein mag.

In der dritten Sitzung war das am häufigsten ausgewählte Erlebnis „Auftauchen früher blockierter Erlebnisse oder Erinnerung" und "Wahrnehmen der Ursache des Problems", wobei diese beiden Erlebnisse in den letzten drei Sitzungen gar nicht genannt wurden. Das Erlebnis „Auftauchen früher blockierter Erlebnisse oder Erinnerung" und "Wahrnehmung der Ursache des Problems" ist Problemaktualisierung, wie ich in dieser Phase erwartet habe.

In der vierten bis achten Sitzung zielt der zweite Schritt auf Auflösung der Konflikte und unterbrochenen Prozesse (unfinished Business) durch aktiven Ausdruck mit kreativen Medien ab. Das Thema in der vierten und fünften Sitzung war "Ausdruck der negativen Gefühle gegenüber sich selbst“. Also, hier wurde die selbstunterbrechende Spaltung (Gefühlsblockierung oder Resignation), die normalerweise in der Gestalttherapie mit der "Zwei-Stühle-Technik“ bearbeitet wird, durch die Gestaltung und das dargestellte Produkt mit kreativen Medien untersucht. Das Thema in der sechsten und siebten Sitzung war Ausdruck der negativen Gefühle gegen bestimmte Personen, nämlich unerledigte oder unterbrochene Prozesse oder Gefühle mit bestimmten Personen. In der Gestaltatherapie wird es mit der „LeerenStuhl-Technik“ bearbeitet. Stattdessen wurde es in dieser Studie durch Gestaltung und das dargestellte Produkt im Bezug auf das Thema bearbeitet. Alle Teilnehmer hatten intensive Einzelarbeit in der Gruppe jeweils in der vierten bis achten Sitzung, wenn sie bereit waren, an dem gegebenen Thema zu arbeiten. d.h. jeder Teilnehmer hatte zweimal die intensive Einzelarbeit in der Gruppe. In der sechsten und siebten ggf. auch achten Sitzung beschäftigten sich die Teilnehmer mit dauerhaften negativen Gefühlen gegenüber bestimmten anderen Menschen, die ihr Elternteil (16 Fälle), ihre Geschwister (2 Fälle) und ihre Freunde (4 Fälle) waren. Eine Teilnehmerin war bis zur 


\section{Diskussion}

achten Sitzung nicht bereit, die negativen Gefühle gegenüber ihrem Vater zu bearbeiten. Erst in der neuten Sitzung konnte sie diese Gefühle zum Ausdruck bringen. Das Erlebnis „Eigenes Problem mit verschiedenen Perspektiven zu sehen“ wurde in der vierten Sitzung am häufigsten genannt. Das Erlebnis „emotionale Expression“ wurde in der fünften Sitzung als hilfreichstes von fünf Teilnehmern am häufigsten genannt. Diese Tendenz trat gleich in der sechsten Sitzung (vier Teilnehmer), siebten Sitzung (fünf Teilnehmer), achten Sitzung (fünf Teilnehmer) und auch neunten Sitzung (drei Teilnehmer) auf. In der fünften Sitzung wurde „Besseres und tieferes Selbstverständnis" von vier Teilnehmerinnen und in der sechsten Sitzung von drei Teilnehmerinnen als hilfreichstes bezeichnet. Nach der ersten Stelle des häufigsten Erlebnisses „emotionale Expression“ kam das Erlebnis „Entwicklung oder Erkennen der Strategie“ gegen Probleme in der siebten Sitzung mit vier Teilnehmerinnen als hilfreichstes hervor. In der achten Sitzung kamen zwei Erlebnisse „Ausprobieren der Strategie" und „Einsicht zu gewinnen" an die zweite Stelle.

Aus diesen Ergebnissen kann man folgern, dass das Programm für Teilnehmer mit dem häufigsten Wirkfaktor „emotionale Expression“ zur Reduzierung von depressiven Symptomen beitragen konnte. Das von Teilnehmern am häufigsten genannte Erlebnis „emotionale Expression“ war 26 mal in dem gesamten Prozess des Programms als hilfreichstes genannt. Davon wurde es in der fünften bis achten Sitzung 19 mal genannt. Da in der vierten Sitzung die Gestaltung mit kreativen Medien stattfand, wurde eher das Erlebnis „Eigenes Problem mit verschiedenen Perspektiven zu sehen" am häufigsten genannt. In der vierten Sitzung erkannten die Teilnehmer ihre Probleme und waren bereit an Problemen $\mathrm{zu}$ arbeiten, da die Probleme aktualisiert wurden. In dem Programm GPD-J wurde die Arbeit mit dem dargestellten Produkt statt der Stuhltechnik eingesetzt, um Selbstspaltung und unterbrochene Gefühle oder Prozesse mit bestimmten Personen zu erledigen. Dieses Interventionsprinzip ist wohl gelungen, wenn man die Ergebnisse der Prozessanalyse mit dem hilfreichsten Erlebnis der Teilnehmer überdenkt. Es zeigt auch, wie bei anderen Behandlungen von Depression in der Gestattherapie bzw. EFT, dass die Interventionsprinzipien mit der „Selbstspaltung" und der „unterbrochenen Gefühle oder Prozess“ mit bestimmten Personen sinnvoll und angemessen zur Prävention von Depression bei Jugendlichen ist. In der neunten bis zehnten Sitzung zielte der dritte Schritt auf die Stärkung zur Selbstunterstützung und Gewinnung eigenen Lebenssinns ab. Diesem Schritt folgte die Integration dessen, was die Teilnehmer in der Therapie tief erlebt haben, damit sie es in ihrem Leben umsetzen können. In der achten Sitzung war das Thema „Ich-Grenzen“. 


\section{Diskussion}

In dieser Sitzung hatten Teilnehmer die Chance, die Restgefühle zum Ausdruck zu bringen, sich damit auseinanderzusetzen und eigenen Grenzen bildlich mit kreativen Medien zu schaffen. An erster Stelle der Häufigkeit des Erlebnisses stand „Besseres und tieferes Selbstverständnis“ und „Einsicht zu gewinnen“ in der neuten Sitzung jeweils mit der Zahl von fünf, „Selbstwertgefühle zu haben“ in der zehnten Sitzung mit der Zahl von fünf gezeichnet. Diese Ergebnissen bestätigten sich, dass die Zielsetzung der Intervention des Programms GPD-J im dritten Schritt nachvollziehbar und sinnvoll war.

In dieser Prozessanalyse wurden vier verschiedenen Hlifsfaktoren herausgefunden; die drei phasenabhängigen Hilfsfaktoren und ein phasenunabhängiger Faktor, der immer kontinuierlich in jeder Phase als hilfreich aufgetreten war. Die in der Anfangsphase vorkommenden Hilfsfaktoren sind "Selbstöffnung“, „Spaß und Erleichterung beim Ausdruck", "Alle haben ähnliche Probleme wie ich", "Auftauchen früher blockierter Erlebnisse oder Erinnerungen“, "Von anderen mich widerspiegeln zu lassen oder berührt zu werden“, „Wahrnehmen der Ursache des Problems“, „Erkennen eigener Bedürfnisse“, „Authentisch oder sich selbt stimmig zu sein“ und „das Gefühl zu haben, verstanden zu werden". Die in der Mittelphase vorkommenden Hilfsfaktoren waren „Hilfe von Anderen zu bekommen“, „Eigenes Problem mit verschiedenen Perspektiven zu sehen“, „Entwicklung oder Erkennen der Strategie“, „Anderen Hilfe zu sein“ und „Nähe zur Therapeutin und der Teilnehmerinnen zu empfinden“. Die in der Endphase vorkommenden Hilfsfaktoren waren "die Gruppe als eins zu erleben“, „Ausprobieren der Strategie“ und „Einsicht zu gewinnen“. Die phasenunabhängigen Hilfsfaktoren waren „Emotionale Expression“, "Spaß bei der Exploration des dargestellten Produkts“, „Interaktion mit der Therapeutin“, „Besseres und tieferes Selbstverständnis“ und "Selbstwertgefühl zu haben“.

Das am häufigsten gewählte Erlebnis im gesamten Verlauf des Programms war „emotionale Expression“ mit 28 Nennungen, dann „besseres und tiefes Selbstverständnis“. Darauf folgen „Einsicht zu gewinnen“ und „Selbstwertgefühl zu haben“. Das am seltensten genannte Erlebnis war „Information zu bekommen“ mit nur einer Auswahl im gesamten Verlauf des Programms. Das Erlebnis „emotionale Expression" gab es ab der dritten Sitzung bis zum Ende, aber besonders in der fünften bis achten Sitzung viel mehr als bei den anderen. Das Erlebnis „Besseres und tiefes Selbstverständnis“ verteilte sich von Anfang bis zum Ende des Programms, aber trat in den letzten zwei Sitzungen gehäuft auf. Das Erlebnis „Einsicht zu gewinnen“ gab es nur in letzten 3 Sitzungen. Das Erlebnis "Selbstgefühl zu haben“ trat in letzten drei Sitzungen verstärkt auf. 


\section{Diskussion}

Yalom (1985) zeigt mit den zwölf therapeutischen Faktoren, dass die Wahrnehmung des Klienten für therapeutische Faktoren von der Entwicklungsphase der Gruppe und den individuellen Variablen des Klienten abhängig ist. In der vorliegenden Arbeit zeigen das die Ergebnisse vom hilfreichsten Erlebnis auch. Das hilfreichste Erlebnis in der Therapie oder Beratung ist als ein Wirkfaktor übersetzbar (Kivlighan et al., 1988; Yalom, 1985). Aber man kann nicht einfach feststellen, welche Faktoren bei welchem Problem wirken, bei wem sie wirken und wie sie bewirken. Es geht in der vorliegenden Untersuchung darum, durch Prozessanalyse und ihre Vorstellung den Programmverlauf und seine Wirfaktoren zu zeigen, damit die Forscher und Leser den Mikroprozess des Programms nachvollziehen können.

\subsection{Entwicklung und Anwendung eines Präventionsprogramms bei psychischen Problemen für die Gesundheitsförderung in dem Kontext "Schule"}

Die meistens der Präventionsprogramme sind kognitiv-verhaltenstherapeutisch und psychoedukativ konzipiert. Ergebnisse von Studien (Clarke et al. 1995) zeigen eindeutig, dass reine psychoedukative Programme in der Depressionsprävention bei Jugendlichen keine Effekte bringen. Das Programm GPD-J wurde mit den psychologischen und erlebnisorientierten Interventionsmaßnahmen auf der therapeutischen Basis von Gestalttherapie und emotionsfokussierter Therapie unter Anwedung von Methoden kreativer Medien entwickelt.

Psychologische Interventionen im Jugendalter stellen in vielerei Hinsicht eine Herausforderung dar und Forschungsprojekten auf diesem Gebiet sind notwendig, haben aber begrenzte Aussagekraft, da insbesondere bei Interventionsstudien mit einer Vielzahl von unspezifischen Effekten (z.B. Therapeutenvariable, kontextuelle Einflüsse) zu rechnen ist. Aber gerade im Jugendalter sind präventive Maßnahmen besonders wichtig und sinnvoll.

In Pubertät und Adoleszenz, einer Phase tiefgreifender Wandlungen, erleben Jugendliche krisenhafte Entwicklungen von körperlichen Umstellungen bis zum Paradigmawechsel im Weltbezug. Das sind Herausforderung, die aber auch ohne krisenhafte Zuspitzung trotz massiver Wandlungen produktiv bewältigt werden können (Ciompi 1982; Resch 1995).

In der vorliegenden Untersuchung haben die weiblichen Adoleszenten an dem Präventionsprogramm GPD-J teilgenommen. Ihre Entwicklungaufgaben und Krisen des Adolszenzalters kennzeichnen die Entwicklung von Identität vs. Identitätskrisen 


\section{Diskussion}

oder Depersonalität, Selbstwert vs. Narzisstische Krisen, Individualität vs. Autonomie, Intimität vs. Beziehungskrisen und Selbstbehauptung vs. Rivalitätskrisen oder Autoritätskrisen. Dieses Entwicklungsalter kann mit zahlreichen Risikoverhaltensweisen einhergehen, z.B. Alkohol- und Drogeneinnahme, Delinquenz, Abnahme der Leistungsmotivation, aggressive Interaktionen, Depression einschließlich Rückzug und Kontaktabbruch. Die Institution Schule sollte diese sollte diese Phänomene nicht vernachlässigen, sondern Wege aufzeigen, wie sie ihre Jugendlichen vor den Gefahren der Risikoverhaltens retten oder im Vorfeld schützen kann.

Bei der Bedarfsanlayse in dieser Untersuchung haben viele Schüler sich für die Teilnahme an dem Programm interessiert, und in der an der Untersuchung beteiligten Schule für Mädchen (10. bis 12 Klasse) hat sich ein Drittel der Schülerinnen selbst für das Programm angemeldet. D.h. die Schüler brauchen nach eigener Einschätzung eine psychologische Beratung, damit sie mit ihren psychischen Problemen besser umgehen können. Aber die Schulsituation entspricht nicht ihren Bedürfnissen. In dieser Schule gibt es zwei Beratungslehrerinnen, die aber meistens wegen des Fachunterrichts unter Müdigkeit leiden, und sich deshalb nicht aktiv betätigen können. Sie und ihre Kollegen bemerken aber immerhin die Notwendigkeit der Beratungskompetenz für Schüler, da es immer mehr Schüler mit psychischen Schwierigkeiten aus verschiedenen Gründen gibt. Als ich in dieser Schule das Programm durchführte, baten mich viele Lehrer um ein Fortbildungsangebot, um sie bei mir sowohl fachliche Kenntnisse im Umgang mit Jugendlichen als auch praktische Methoden zur Beratung von Jugendlichen erlernen zu können. Sind die Interessen von Lehrern an besserer Kompetenz zur Befriedigung der Bedürfnisse von Schülern nach psychologischer Hilfe nicht Hinweis genug? Die Antwort auf diese Fragestellung muss die Bildungspolitik verantworten. Bildungsqualität hängt $\mathrm{ab}$ von Lehrerkompetenz. Wenn Lehrer nicht genügend kompetent sind, scheitert die Bildung. Aber Lehrer können nicht in allen Bereichen und nicht in jeder Situation kompetent sein. Lehrer brauchen Beratungskompetenz aber auch selber Beratung.

Meiner Meinung nach sollte in jeder Schule ein Zentrum für Gesundheitsförderung und Beratung für Angehörige der Schule zur Verfügung stehen, wo Fachleute immer da sind für Schüler, Eltern und Lehrer, aber nicht unterrichten. Hier finden Beratungsausbildung für Lehrer, Beratungsangebote für Schüler in Zusammenarbeit mit Eltern und Durchführung verschiedener Programme zum Zweck der Prävention von bestimmten bzw. wahrscheinlichen Störungen und auch verschiedene gesundheitsfördernde Programme statt. 


\section{Diskussion}

Seit dem PISA-Schock versuchen deutsche Schulen einschließlich der Schulsysteme sich zu ändern, bieten teilweise "Ganztags-Schulen“ an. Aber man muss dabei auch gut überlegen, wofür die Schulen den ganzen Tag geöffnet werden sollten, und wie diese Ganztags-Schulen die Lebenswelt der Schüler beeinflussen könnten. Wenn es darauf abzielt, dass die Schüler bessere Noten als zuvor oder bessere Leistungen im Vergleich $\mathrm{zu}$ anderen Ländern haben, muss man nicht nur erwartete Wirkungen sondern auch Nachwirkungen vorher berücksichtigen. Setzen sich die Schüler unter Konkurrenzdruck, haben sie keine Zeit zum Genuss beim Lernen und Leben, können sie imstande sein, langsam ihre Lust nicht nur auf das Lernen sondern auf das Leben zu verlieren. Da Lehrerqualität also nicht nur fachliche Kompetenz erfordert sondern auch die Kompetenz auf Schüler mit Schwierigkeiten aufmerksam zu werden und mit ihnen umgehen zu können, ist „Beratungskompetenz“ für Lehrer notwendig.

Krause (2003) stellt drei Gruppen von Anlässen von Beratung dar: Informationsbedarf, psychische Destabilisierung oder inadäquate Belastung bzw. Entlastung und Prävention zur Verhinderung von antizipierten Problemen. Diese drei Bereiche sollten mit dem bestimmten Ziel und nach Bedarf von nicht nur betroffenen Schülern sondern auch helfenden Lehrern im Sinne von Schulberatung noch verfeinert und systematisch strukturiert werden. Aus diesem Grund sollten die Beratungslehrer und Kliniker noch viel zur Verbesserung oder Renovierung der Schulberatung beitragen, z.B. Entwicklung von verschiedenen Beratungsprogrammen bei unterschiedlichen psychischen Problemen und für Prävention in unterschiedlichem Schweregrad je nach psychischen Problemen. Die Institution „Schule“ sollte für jeden Schüler ein Raum sein, wo er seinen Traum entfalten kann und nicht sein Trauma erleben muss.

\subsection{Relevanz der Prävention von Depression bei weiblichen Jugendlichen}

Verschiedene Studien legen nahe, dass in der Jugendzeit bzw. nach der Pubertät Mädchen deutlich häufiger von Depression betroffen sind als Jungen (Cyranowski et al. 2000, Groen et al. 1997), Depressionen bei 20,4\% der 14- bis 24-jährigen Mädchen (Wittchen et al., 1994) und ab dem Alter von 15 Jahren die Rate depressiver Mädchen cirka doppelt so hoch ist als Junge (Hankin et al.1998, Lewinsohn et al. 1998, MaGee et al. 1992). Bei depressiven Mädchen scheint es einen Zusammenhang zwischen der negativen Wahrnehmung des eigenen Körpers und dem defizitär entwickelten Selbstwertgefühl zu geben (Lewinsohn et al., 1994). Andere Forschungsergebnisse belegen, dass bei weiblichen Jugendlichen ein stärkerer und langanhaltender Einfluss 
von schulspezifischen Stressoren aus der Frühadoleszenz auf depressive Symptome in der Spätadoleszenz besteht (Seiffge-Krenke \& Klessinger 2001).

Wie erwähnt, verursachen depressive Störungen bei Jugendlichen starke soziale Beeinträchtigungen nicht nur in der Gegenwart sondern auch weitere chronische psychische Störungen bis ins Erwachsenenalter. Dennoch sind Depressionen bei Jugendlichen in der kurzen Zeit der Untersuchungsgeschichte kaum ans Licht gekommen, da einerseits Depressionen internalisierte Symptommerkmale zeigen und anschließend häufig mit anderen offenkundigen Problemen zusammenhängen, z.B. somatischen Beschwerden, Schulversagen und Verhaltensproblemen, sodass depressive Störungen selbst wenig ernsthafte Aufmerksamkeit von Eltern oder Erziehern bekommen. Da sich andererseits die Jugendlichen gerade körperlich und emotional in der Entwicklungsphase befinden, mit der sie sich auseinandersetzen müssen und in der sie sich fremd fühlen, ist es für Jugendliche schwer zu erkennen, was geschieht und ob es normal ist oder nicht.

Bei weiblichen Jugendlichen mit Depression ist die Befindlichkeit noch komplizierter aufgrund der Pubertät. Hierbei spielen zusätzlich geschlechtsspezifische Sozialisationserfahrungen und Rollenerwartungen der Gesellschaft eine große Rolle für weiblichen Jugendliche. Die negativen geschlechtsspezifischen Rollenerfahrungen führen zu sozialem Rückzug als Reaktionsalternative durch internale Attribution, indem sie sich nicht ausdrücken und nicht zu unterscheiden vermögen, was sie wollen und was nicht. Dabei spielt der Druck, den die Gesellschaft und die Eltern durch hohe Leistungeserwartungen ausüben, eine enorme Rolle in Korea. Das Studium ihres Kindes an einer der besten Universitäten ist das Ziel vieler koreanischer Familien, die Zulassungsbestimmungen sind aber sehr streng, da die Unversitäten mehrstufig klassifiziert sind.

Es ist deshalb dringend notwendig, insbesonders die weiblichen Jugendlichen vor Depressionen zu schützen, auch im Hinblick au die Erkrankung des zukünftigen Nachwuchsses, weil sie später als depressive Mütter ihre Kinder beeinflüssen könnten. Protektive Faktoren sollten so früh als möglich verstärkt werden. Im diesem Sinne ist das hier entwickelte Programm zur Prävention von Depression inhaltlich ein Gesundheitsförderungsprogramm. Aber in Abgrenzung zu heute üblichen SettingDesigns in der Gesundheitsförderung wendet sich dieses Programm explizit an eine Risikopopulation, sozusagen an selektive bzw. indikative Gruppen mit Depressionen. 


\section{Diskussion}

\subsection{Einschränkungen der Untersuchung und Forschungsausblick}

Die vorliegende Studie hat einige nicht unerhebliche Grenzen, auf die hingewiesen werden muss.

Diagnosen : Eine wichtige Einschränkung betrifft die „Diagnosen“, die nur auf Rating beruhen und nicht auch auf direkten Beobachtungen und die vor allem nicht kinderund jugendpsychiatrisch abgesichert sind. Es ist also denkbar, dass die Depressionssymptomatik bei dem einen oder anderen Jugendlichen differenzialdiagnostisch einem anderen Störungsbild zugeordnet werden muss oder gar nicht als Störung im diagnostischen Sinne $\mathrm{zu}$ beurteilen ist. Da die Übereinstimmung der Information hinsichtlich der Häufigkeit und des Schweregrades von Depression bei Kindern und Jugendlichen gering ist (Angold et al., 1987; Essau, 2002), wäre es vorteilhaft gewesen, Daten aus verschiedenen Quellen, z.B. Beurteilungen von Eltern oder Bezugspersonen, heranzuziehen.

Erhebungsinstrumente zur Evaluation: Auch die Frage der „Erhebungsinstrumente“ ist offen. In vorliegender Arbeit wurden standardisierte Selbstbeurteilungsfragebögen verwandt. Die vorhandenen Fragebögen sind entweder für Kinder im Alter von 16 bis 17 Jahren oder für Erwachsene im Alter von mindestens 20 Jahren. Wegen mangelnder Erhebungsinstrumente für 16- bis 18-jährige Jugendliche wurden hier die Fragenbögen für Erwachsene eingesetzt. Daher ist es dringend nötig, verschiedene Messinstrumente für Jugendliche im Alter von 17 bis 19 zu entwickeln.

Da die Erhebung verdeckter Prozesse (z.B. Empfindungen von Depression, Schuld, Hoffnungslosigkeit und Suizidgedanken) und schwer zu beobachtender somatischer Symptome (z.B. Schlaflosigkeit, Appetitverlust) für Kinder und Forscher eine Herausforderung darstellen (Reynold 1994; Essau 2002), haben Fragebögen gewisse Vorteile bei der einfachen und günstigen Erhebung von Symptomen depressiver Störungen. Dennoch ist es denkbar, dass die Ergebnisse der Untersuchung anderer Dimensionen von Teilnehmer erhoben werden könnten, wenn nicht nur Selbstbeurteilung sondern auch Fremdbeurteilung zur Identifizierung der Symptome beiträgt.

Generalisierung der Ergebnisse : Auch die Frage der Generalisierung der Ergebnisse ist offen. Wenn menschliche Individuen mit bestimmten Störungsbildern mit dieser Therapie behandelt werden, bessern sich die Störungsbilder (Zepf \& Hartmann, 2002, S, 279). Um dies generalisieren $\mathrm{zu}$ können, bedarf es weiterer Forschung mit größeren Probandengruppen. Es sollte untersucht werden, ob das Programms GPD-J in verschiedenen Altersstufen und Gruppen mit unterschiedlichen Ausprägungen von 


\section{Diskussion}

Depression effektiv ist und ob die Wirksmkeit des Programms auch bei Jungen gültig ist, z.B. in universalen Präventionsgruppen in der Grundschule im Vergleich von Jungen und Mädchen im Alter zwischen 15 bis 17 Jahren. Zur Bestätigung der Generalisierungsannahme müsste das Forschungsdesign genauer spezifiziert werden. Zum Vergleich der Wirksamkeit mit unterschiedlichen Behandlungsmaßnahmen wäre ein Forschungsdesign in Dreiergruppen geeignet: Zwei Versuchsgruppen, in der unterschiedliche Behandlungen durchgeführt werden, und eine Kontrollgruppe ohne Intervention.

Variable „Durchführerin“ : Das Projekt in der vorliegenden Arbeit hat die Autorin allein durchgeführt, weshalb die Wirksamkeit des Programms anders sein könnte, wenn mit einem Kotherapeuten gearbeitet wird oder ein erfahrener Therapeut das Programm durchführt. Insofern könnte man auch argumentieren, dass es besonders bemerkenswert ist, dass das von der Verfasserin selbständig erprobte Programm Effekte nachgewiesen hat. Deshalb scheint es lohnenswert zu überprüfen, ob das Programm gleich oder unterschiedlich wirksam ist, wenn es von anderen durchgeführt wird.

Variable „Teilnehmer": Es ist bekannt, dass „Erwartungen und Motivationen“ von Klienten oder Patienten ein wichtiger Faktor des Erfolgs einer Beratung oder einer Therapie sind. Deshalb ist es möglich, dass, wenn das Programm mit Jugendlichen, die nicht hoch motiviert sind, durchgeführt würde, andere Ergebnisse als bei dieser Studie zu erwarten wären. Auch in einer Jungengruppe könnten die Ergebnisse anders sein als bei der Gruppe mit Mädchen in dieser Studie.

Variable „Individuumzentrierte Sicht": Das Programm ist nicht systemisch oder familientherapeutisch, sondern eher individuumzentriert, d.h. in der Arbeit wurde Elternarbeit ausgeschlossen. Da Eltern immer noch die häufigsten Bezugspersonen der Heranwachsenden sind, kommt systemischen oder familientherapeutischen Kompetenzen in der Arbeit mit Kindern und Jugendlichen auf jeden Fall eine wesentliche Rolle zu. Meiner Ansicht nach ist es bei 16- bis 18-jährigen Jugendlichen zunächst sinnvoll, individuumzentriert $\mathrm{zu}$ arbeiten, wenn Veränderungen ohne Elternarbeit vorstellbar sind. Tatsächlich zeigten die Ergebnisse mit dem Programm GPD-J den Erfolg der individuumzentrierten Interventionen. Dennoch ist es lohnenswert, zu forschen, wie ein Präventionsprogramm bei Jugendlichen mit beiden Ansätzen, also systemisch und individuumzentriert, integrierend konzipiert werden kann und in welcher Form diese Einbeziehung dann genauer erfolgen soll und mit welchen möglichen Auswirkungen auf die einbezogenen Personen dies geschieht. 


\section{Diskussion}

Variable „Hilfe nach dem Programm“: Die Teilnehmerinnen an der Versuchsgruppe haben nach dem Programm zwei Selbsthilfegruppen gebildet, um sich selbst und anderen Hilfe zu geben. Sie wollen in der Schule einen Club für „Peer-Counseling“ mit der Hilfe von zwei Beratungslehrerinnen, die in dieser Schule tätig sind, entwickeln, damit sie anderen mit der kollegialen Beratung helfen können. Sie haben sich bei der Therapeutin im Internet gemeldet, wenn sie Hilfe brauchten. Da die Therapeutin den Teilnehmerinnen beider Gruppen, Versuchs- und Kontrollgruppe, ihre E-mailadresse gegeben hat, können alle Teilnehmerinnen Kontakt aufnehmen. Jedoch haben die Teilnehmerinnen der Kontrollgruppe nur viermal Kontakt aufgenommen, während sich die Teilnehmerinnen der Versuchsgruppe oft gemeldet haben. Es wäre lohnenswert, zu erforschen, ob die Wirksamkeit des Programms von solchen Kontakten nachhaltig beeinflusst werden kann.

Prozessanalyse: Die meisten bisher bekannten Untersuchungen zum hilfreichen Erlebnis in der Therapie bzw. Beratung waren auf Erwachsene angelegt. In der vorliegenden Arbeit wurde das hilfreiche Erlebnis bei Jugendlichen untersucht, um Wirkfaktoren des Programms im Sinne der Prozessanalyse herauszufinden. Das Erlebnis hatten Teilnehmerinnen schriftlich subjektiv eingeschätzt und der Text wurde dann analysiert. Obwohl bei der Analyse das Videoband zusätzlich verfügbar war, wurde nicht objektiv untersucht, wie das hilfreiche Erlebnis stattfindet und wie sich die Teilnehmerin dabei verhält. Dashalb erscheint es auch für die zukünftige Forschung erforderlich, das hilfreiche Erlebnis in der Therapie bzw. Beratung mit auch objektiven Untersuchungsmethoden, z.B. Analyse der Sitzungsprotokolle zu kombinieren.

Gruppierungsprozess und ethische Probleme: Die Schülerinnen, die sich selbst angemeldet hatten, bekamen nach der Diagnostik die Chance, einen von zwei Terminen für die Teilnahme an dem Projekt auszuwählen. Am Anfang der Vorbereitung des Projekts wurden sie schon darüber informiert, dass nicht alle an dem Programm teilnehmen können, sondern ein Teil von ihnen in die Kontrollgruppe ohne Intervention eingeteilt werden würde. Wenn sie nicht damit einverstanden waren, durften sie an dem Projekt nicht teilnehmen. Schließlich wurden 44 Teilnehmerinnen mit ihrer Einverständniserklärung nach der Auswahl des Termins in zwei Gruppen, also Versuchs- und Kontrollgruppe, zufällig eingeteilt. In diesem Prozess zur Gruppierung wäre es möglich gewesen, dass die Teilnehmerinnen in der Kontrollgruppe enttäuscht gewesen wären, sodass ihre Stimmung die Ergebnisse beeinflussen könnte. Und umgekehrt wäre es auch denkbar, dass die Hoffnungs- und Geborgenheitsgefühle bei der Versuchsgruppe als Placeboeffekt für die Ergebnisse 


\section{Diskussion}

wirken könnten. In zukünftigen Forschungen ist es erfoderlich, zu ermitteln, wie man solche ethischen Probleme in der Forschung beseitigen kann und wie die empirische Therapie- und Beratungsforschung mit unterschiedlichen Behandlungsmaßnahmen den Placeboeffekt kontrollieren können.

Qualifizierung des Programms : Das Programm GPD-J wurde von der Autorin unter der Supervision einer Integrativen Therapeutin und einer Gestaltherapeutin entwickelt. Obwohl die Wirksamkeit des Programm bei Jugendlichen mit Depressionen sehr hoch war, sollte es mit erweiterten Anwendungen qualifiziert werden. Darüber hinaus ist es auch erforderlich zu erforschen, ob das Programm in der Ausbildung für Beratungslehrer eingesetzt werden kann und sich ergänzen und verbessern lässt, indem es von Beratungslehrern in der Schulsituation angewandt wird. 


\section{Zusammenfassung}

\section{Zusammenfassung}

Mit dieser Studie wurde der Versuch unternommen, ein Programm für indikative Prävention von Depressionen bei Jugendlichen basierend auf Gestaltgruppenarbeit mit kreativen Medien zu entwickeln und seine Wirksamkeit bei Depressionen sowie hilfreiche Erlebnisse während des Therapieprozesses zu untersuchen.

Das Programm GPD-J (Gestalttherapeutische indikative Prävention von Depression bei Jugendlichen) wurde auf den gestalttherapeutischen und emotionsfokussierten therapeutischen Grundlagen mit dem methodischen Einsatz von kreativen Medien kombiniert. Die Behandlungsgrundlage des Programms gegen Depression stammt aus dem Manual EFT. Es besteht aus 10 therapeutischen Sitzungen, in denen sich die Teilnehmer jeweils zwei Zeitstunden mit verschiedenen kreativen Medien beschäftigen. Zur Durchführung des Programms wurden 44 Schülerinnen, die sich frei angemeldet hatten, im Alter zwischen 16 und 18 nach subklinischer depressiven Symptomatik unter den Kriterien DSM-VI und nach der Werten BDI (Becks Depression Inevntar) ausgewählt. Die Schülerinnen wurden in zwei Gruppen, also Versuchs- und Kontrollgruppe, zufällig eingeteilt. Die beiden Gruppen waren bezogen auf die depressive Symptomatik homogen.

Zur Prüfung der Wirksamkeit des Programms GPD-J wurden Versuchs- und Kontrollgruppe vor Beginn der Intervention, nach Abschluss der Intervention und dann noch einmal drei sowie sechs Monate danach psychologisch getestet. Zusammenfassend konnten für die Wirksamkeit des Programms GPD-J mit Hilfe der psychometrischen Tests eine signifikante Reduzierung der depressiven Symptome sowie der psychischen Beschwerden und eine signifikante Steigerung des psychischen Wohlbefindens festgestellt werden. Aufgrund der Ergebnisse kann zudem die Aussage getroffen werden, dass die Versuchsgruppe während der Intervention klare Fortschritte machte, und dass im Vergleich zur Kontrollgruppe erhebliche Verbesserungen in den Bereichen Depression, Depressivität, psychische Beschwerden und psychisches Wohlbefinden erreicht wurden. Weiterhin zeigte sich eine signifikante Verbesserung bei der Versuchsgruppe in den zwei Follow-Up-Messzeitpunkten nach der Intervention im Vergleich zum Messzeitpunkt vor der Intervention in allen o.g. Bereichen, während es bei der Kontrollgruppe keine Verbesserung gab. Die langfristigen Effekte nach drei und sechs Monaten erwiesen sich als statistisch durchgehend signifikant. Die stärksten Effekte bei der Versuchsgruppe waren direkt nach der Intervention zu beobachten. Während des Programms haben die stärksten 


\section{Zusammenfassung}

Effekte bei der Versuchsgruppe in der fünften Sitzung stattgefunden, da hier die BDIWerte dramatisch gesunken waren.

Darüber hinaus wurde im Therapieprozess untersucht, welches Erlebnis während des Programmverlaufs für Teilnehmer ein hilfreiches Erlebnis war. Die von den Teilnehmerinnen ausgewählten Erlebnisse wurden qualitativ analysiert, um Wirkfaktoren herauszufinden. In dieser Prozessanalyse wurden vier verschiedene Hilfsfaktoren herausgefunden. Drei phasenabhängige Hilfsfaktoren - in der Anfangsphase, der Mittelphase und der Endphase vorkommende Faktoren - und ein phasenunabhängiger Faktor, der immer kontinuierlich in jeder Phase als hilfreich aufgetreten war. Die Erlebnisse wurden nach der qualitativen Analyse kategorisiert, und zwar nach „Expression“ (Kategorie 1), „Kontakt mit anderen“ (Kategorie 2), „Problem“ (Kategorie 3) und „Selbstwahrnehmung“ (Kategorie 4). Die hilfreichen Erlebnisse wurden nach den vier Kategorien sortiert und ihre Häufigkeit analysiert. Die Ergebnisse zeigen, dass am häufigsten "Selbstwahrnehmung“ (Kategorie 4), dann der Reihe nach „Expression“ (Kategorie 1), „Kontakt mit anderen“ (Kategorie 2) und „Problem“ (Kategorie 3) im gesamten Verlauf des Programms für die Teilnehmerinnen hilfreich waren. Die Kategorie 1 „Expression“ wurde in der ersten bzw. fünften Sitzung am häufigsten genannt. Die Kategorie 2 „Kontakt mit anderen“ wurde durchgehend von Anfang an bis zum Ende des Verlaufs des Programms, aber am häufigsten in der fünften Sitzung benannt. Die dritte Kategorie „Problem“ ist am häufigsten in der siebten Sitzung während des Verlaufs erwähnt worden. Die Kategorie 4 "Selbstwahrnehmung“ wurde ab der siebten Sitzung bis zum Ende des Programms immer häufiger genannt, zeigte sich also am häufigsten in der zehnten Sitzung.

Insgesamt konnte in der Untersuchung nachgewiesen werden, dass das Programm GPD-J als indikative Präventionsmaßnahme bei Depressionen im Jugendalter wirksam sein kann. 


\section{Literaturverzeichnis}

\section{Literaturverzeichnis}

Amendt-Lyon, N. (1999): Kunst und Kreativität in der Gestalttherapie. In: R. Fuhr, M. Sreckovic \& M. Gremmler-Fuhr (Hrsg.): Handbuch der Gestalttherapie. S. 857-878. Göttingen: Hogrefe.

Amendt-Lyon, N. (1999): Mit Ihnen schreibe ich heute Geschichte. In: R. Hutterer Krisch, I. Luif \& G. Baumgartner (Hrsg.): Neue Entwicklung in der integrativen Gestalttherapie. S. 149-168. Wien: Facultas.

Amendt-Lyon, N. (2003): Auf dem Weg zu einem gestalttherapeutischen Konzept zur Förderung des schöpferischen Prozesses. In: M. Spagnuolo Lobb \& N. AmendtLyon. (Hrsg.): Creative License : The Art of Gestalt Therapy. New York: Springer. dtsch. (2006). Die Kunst der Gestaltherapie : eine schöpferische Wechselbeziehung. S. 7-26. Wien:Springer.

Amendt-Lyon, N. \& Hutterer-Kirsch, R. (2000): Diagnostik in der Gestalttherapie. In: A.R. Laireiter (Hrsg.): Diagnostik in der Psychotherapie. S. 179-192. Wien, NewYork: Springer.

Anderson, J.C. \& McGee, R. (1994): "Comorbidity" of depression in child and adolescents. In: W.M. Reynolds \& H.F. Johnston (Eds.): Handbook of depression in children and adolescents, S. 581-601, New York: Plenum

Anderson, J.C., Williams, S., McGee, R. \& Silvia, P.A. (1987): DSM-III disorders in preadolescent children. Prevalence in a large sample from the general population. Archives of General Psychiatry, 44, 69-76.

APA-American Psychiatric Association (1996): Diagnostic and Statistical Manual of Mental Disorders, Fourth Edition: Primary Care Version, DSM-IV-PC. Washington, American Psychiatric Association.

APA-American Psychiatric Association (2000): Diagnostic and Statistical Manual of Mental Disorders, Fourth Edition : Text Version, DSM-IV-TR. Washington, American Psychiatric Association.

Argyle, M. (1975): Bodily Communication. London: Methun. dtsch: (1979). Körpersprache und Kommunikation. Paderborn: Junfermann Verlag.

Armbruster, P. \& Kazdin, A.E. (1994): Attribution in child psychotherapy. Adv Clinical Child Psychology, 16, 81-108.

Badier, A. (1999): Gestalttherapie mit Kindern und Jugendlichen. In : R. Fuhr, M. Sreckovic \& M. Gremmler-Fuhr (Hrsg.): Handbuch der Gestalttherapie. S. 953-964. Göttingen: Hogrefe. 


\section{Literaturverzeichnis}

Baer, U. (2004): Gefühlssterne, Angstfresser, Verwandlungsbilder. 3. Aufl. NeukirchenVluyn:Affenkönig.

Bandler, R. \& Grinder, J.. (1975): The structure of magic. Palo Alto, CA: Science and Behavior Books

Baulig, I. \& Baulig, V. (2002): Praxis der Kinderpsychotherapie. Bergisch Gladbach: EHP.

Beardslee, W.R., Wright, E.J., Salt, P., Drezner, K., Gladstone, T.R. Versage, E.M. Rothberg, P.C. (1997): Examination of children's responses to two preventive intervention strategies over time. Journal of the American Academy of Child and Adolescent Psychiatry, 36, 196-204.

Beisser, A. (1970): The paradoxical theory of change. In : J. Fagan \& I. Shepherd (Eds.): Gestalt therapy now. S. 77-80. New York : Harper.

Beutler, L.E., Eagle, D, Oro-Beutler, M.E., Daldrup, R. \& Meredith, K.(1986): Inability to Express Intense Affect: A Common Link Between Depression and Pain? Journal of Consulting and Clinical Psychology, 54/6, 752-759.

Beutler, L.E., Engle, D, Oro-Beutler, M.E. \& Daldrup, R. (1986): Inability to Express Intense Affect: A Common Link Between Depression and Pain? Journal of Consulting and Clinical Psychology, 54/6, 752-759.

Birmaher, B., Ryan, N.D., Williamson, D.E., Brent, D.A., Kaufman, J., Dahl,R.E., Perel, J. \& Nelson, B. (1996): Childhood and adolescent depression: A review of the past 10 years. Part I . Journal of the American Academy of Child and Adolescent Psychiatry, 35, 1427-1439.

Blankertz, S. (2004): Gestalttherapeutische Diagnostik in Beratung, Therapie und Coaching, Gestaltkritik, 1. Onlinequelle : http://www.gestalt.de/blankertz

Blankertz, S. \& Doubrawa, E. (2005): Lexikon der Gestalttherapie. Wuppertal: Peter Hammer Verlag.

Blom, R.(2006): The Handbook of Gestalt Play Therapy. London, Philadelphia: Jessica Kingsley.

Boulet, D.B., Souliere, M.D., Sterner, I. \& Nadler, W.P. (1992): Development of Category System of Good Moments in Gestalt Therapy. Psychotherapy, 29/4, 554-563. Böker, H. (Hrsg.) (2000): Depression, Manie und schizoaffective Psychosen. 3.Aufl. Gießen: Psychosozialverlag

Briere, J.N. (1992): Child abuse trauma. Newberry Park: Sage.

Brooks, C. V. (1974): Sensory Awareness. The Rediscovery of Experiencing. New York: The

Viking Press. dtsch: (1979): Erleben durch die Sinne. Paderborn : Junfermann Verlag.

Bukstein, o.G., Glancy, L.J. \& Kaminer, Y. (1992): Patterns of affective comorbidity in a 


\section{Literaturverzeichnis}

clinical population of dually diagnosed adolescent substance abusers. Journal of the American Academy of Child and Adolescent Psychiatry, 31, 1041-1045.

Bünte-Ludwig, C. (1985): Gestalttherapie-Integrative Therapie. Leben heißt Wachsen. In : H.G.Petzold (Hrsg.): Wege zum Menschen. Bd1. S. 217-307. Parderborn: Junfermann.

Burke, K., Burke, J., Regier, D. \& Rae, D. (1990): Age at onset of selected mental disorders in five community populations. Archives of General Psychiatry, 47, 511-518.

Burley, T. (2003): Die Neurowisscheschaft der Kreativität : Eine gestalttheapeutische Perspektive. In : M. Spagnuolo Lobb \& N. Amendt-Lyon. (Hrsg.): Creative License : The Art of Gestalt Therapy. New York: Springer. dtsch. (2006): Die Kunst der Gestalttherapie : eine schöpferische Wechselbeziehung. S. 95-106. Wien:Springer.

Burow, O.A. (1993): Gestaltpädagogik: Trainingskonzepte und Wirkungen. Paderborn: Junfermann.

Buttollo, W. \& Maragkos, M. (1999): Gestalttherapie und empirische Forschung. In : R. Fuhr, M. Sreckovic \& M. Gremmler-Fuhr (Hrag.): Handbuch der Gestalttherapie. S. 1091-1120. Göttingen: Hogrefe.

Clarke, G.N., Hawkins, W., Murphy, M., Sheeber, L.B. (1993): School-based prevention of depressive symptomatology in adolescents : Finding from two studies. Journal of Adolescent Research, 8, 183-204.

Clarke, G.N., Hawkins, W., Murphy, M., Sheeber, L.B., Lewinsohn, P.M. \& Seeley, J.R. (1995): Targeted prevention of unipolar depressive disorder in a at-risk sample of high school adolescents : A randomized trial of a group cognitive intervention. Journal of the American Academy of Child and Adolescent Psychiatry, 34, 312-321.

Clarke, K.M \& Greenberg, L.S. (1986): Differential Effects of the Gestalt Two-Chair Intervention and Problem Solving in Resolving Decisional Conflict. Journal of Counseling Psychology, 33/1, 11-15.

Clarkson, P. (2004): Gestalt Counseling in Action, 3. Edition. London: Sage

Clarkson, P. \& Mackewn, J. (1993): Fritz Perls. London: Sage

Compas, B.E., Grant, K.E. \& Ey, S.E. (1994): Psychosozial stress and child and adolescent depression: Can we be more specific? In W.M. Reynold \& H.F. Johnson(Eds.): Handbook of depression in children and adolescents. New York: Plenum

Conoley, C., Conoley, J.V., Naconell, J.A. \& Kimzey, C.E. (1983): The effect of the ABC's of rational emotive Therapy and the empty chair technique of Gestalt therapy on anger reduction. Psychotherapy: Theory, Research, and Practice, 20, 112-117.

Cyranowski, J.M., Frank, E., Young, E. \& Shear, M.K. (2000): Adolescent onset of the gender difference in lifetime rates of major depression : A theoretical model. 


\section{Literaturverzeichnis}

Archives of General Psychiatry, 57, 21-27.

Daldrup, R.J., Beutler, L.E., Engle, D. \& Greenberg, L.S. (1988): Focused expressive psychotherapy: Freeing the over controlled patient. New York: Guilford.

Daniels, V. (2004): Dimensions of Dialogue. Gestalt, 8/2. in Online : http://www.ggej.org/8-1/corner.html.

Delisle, G. (1991): A Gestalt pespective of personality disorders. British Gestalt Journal, 1, 42-50.

Doubrawa, E. \& Staemmler, F.M. (Hrsg.) (2003): Heilende Beziehung : Dialogische Gestalttherapie. 2. erweiterte Auflage. Wuppertal: Peter Hammer Verlag.

Dreitzel, H.P. (1995): Emotion in der Gestalttherapie - ihre Bedeutung und Handhabung im therapeutischem Prozess. In: H.G. Petzold (Hrsg.): Wiederentdeckung des Gefühls. S. 493-517. Parderborn : Junfermann.

Edwards,M. (2001): Jungian Analytic Art Therapy. In : J.A.Rubin (Eds.): Approaches to Art Therapy (2.Ed.), S. 81-94. Philadelphia: Brunner-Routledge.

Elliot, R. (1999): Prozess-Erlebnisorientierte Psychotherapie- Ein Überblick: Teil1. Psychotherapeut, 44, 203-213.

Elliot, R. (1999): Prozess-Erlebnisorientierte Psychotherapie- Ein Überblick: Teil2. Psychotherapeut, 44, 340-349.

Essau, C. A. (2002): Depression bei Kindern und Jugendlichen. München: Reinhardt.

Essau, C.A \& Petermann, U. (2000): Depression. In : Petermann, F.(Hrsg.): Lehrbuch der klinischen Kinderpsychologie und -psychotherapie. S. 291-324. Göttingen:Hogrefe.

Essau, C.A., Conradt, J. \& Petermnn, F. (2000): Frequensy, comorbidity, and psychological impairment of depressive disorders in adolescents. Journal of Adolescent Research, 15, 470-481.

Essau, C.A., Karpinski, N.A., Petermann, F. \& Conradt, J. (1998a): Häufigkeit ind Komorbidität psychischer Störungen bei Jugendlichen: Ergebnisse der Bremer Jugendstudie. Zeitschrift für Klinische Psychologie, Psychiatrie und Psychotherapie, 46, 105-124.

Essau, C.A., Karpinski, N.A., Petermann, F. \& Conradt, J. (1998a): Häufigkeit ind Komorbidität und psychosoziale Beeinträchtigungvon Depressiven Störungen bei Jugendlichen : Ergebnisse der Bremer Jugendstudie. Zeitschrift für Klinische Psychologie, Psychiatrie und Psychotherapie, 46, 316-329.

Fagan, J. \&Shepherd, I. (Eds.) (1970): Gestalt therapy now. New York: Harper.

Fagan, J., Lauver, D., Smith, S. Deloach, S. Katz, M. \& Wood, E. (1974): Critical incidents in the empty chair. The Counseling Psychologist, 4, 33-42.

Fäh,M. \& Fischer, G. (1998): Sinn und Unsinn in der Psychotheraieforschung. Gießen: 


\section{Literaturverzeichnis}

Psychosozial Verlag.

Feder, B. (1994): Die interagierende Gestalttherapiegruppe als fruchtbarer Boden für Wachstum. In : C. Freiler, D. Ventouratou-Schmetterer, C. Reiner-Lawugger \& R. Bösel (Hrsg.): 100 Jahre Fritz Perls : Tagungsband der internationalen Psychotherapietagung der Fachsektion für Integrative Gestalttherapie ÖAGG. S. 176-190. Wien: Facultas-Universitätsverlag.

Fend, H. (2000): Entwicklungspsychologie des Jugendalters. Opladen: Leske+Budrich.

Fischer, G., Frommer, J. \& Klein, B. (1998): Qualitative Kriterien zur Bewertung des

Therapieerfolgs. In: Fäh, M. \& Fischer, G. (Hrag.): Sinn und Unsinn in der Therapieforschung. S. 167-177. Gießen: Psychosozial Verlag.

Fittkau, B. (2003): Zum Stellenwert von Dianostik in der Pädagogischen Beratung. In C.

Krause, B. Fittkau \& U. Thiel (Hrsg.): Pädagogische Beratung. S. 51-72. Pardeborn: Ferdinand Schöningh.

Fleming, J.E., Boyle, M.H. \& Offord,D.R. (1993): The outcome of adolescent depression

in the Ontario Child Health Study Follow-Up. Joumal of the American Academy of Child and Adolescent Psychiatry, 32, 28-33.

Frambach, L. (1993): Identität und Befreiung in der Gestalttherapie. Petersberg: Vianova.

Franck, J. (1997): Gestalt - Gruppentherapie mit Kindern. Freiamt: Arbor Verlag.

Freiler, C., Ventouratou-Schmetterer, D., Reiner-Lawugger, C. \& Bösel, R. (Hrsg.)

(1994): 100 Jahre Fritz Perls : Tagungsband der internationalen Psychotherapietagung der Fachsektion für Integrative Gestaltherapie ÖAGG. Wien: Facultas-Universitätsverlag.

Frohne-Hagemann, I. (Hrsg.) (1999): Musik und Gestalt, Göttingen: Vandenhoeck \& Ruprecht.

Frommann, A. (1990): Was geschieht eigentlich in Beratungen? Beratung zwischen

Kunst und Methode. In: W-J. Brunner \& W. Schönig(Hrsg.): Theorie und Praxis von Beratung: pädagogische und psychologische Konzepte. S. 28-40, Freiburg in Breisgau: Lambertus.

Frühmann, R. (1985): Poesie - ein Weg zu sich selbst. Metareflexionen zue Überwindung von Entfremdung durch poetisches Sagen. In: H.G. Petzold \& I. Orth(Hrsg.): Poesie und Therapie : Über die Heilkraft der Sprache. S. 219-242. Paderborn: Junfermann Verlag.

Frühmann, R. (1994): „Hot-Seat“ und „Empty-Chair“. In: C. Freiler, D. VentouratouSchmetterer, C. Reiner-Lawugger \& R. Bösel (Hrsg.): 100 Jahre Fritz Perls : Tagungsband der internationalen Psychotherapietagung der Fachsektion für Integrative Gestalttherapie ÖAGG. S. 139-154. Wien: Facultas-Universitätsverlag. 


\section{Literaturverzeichnis}

Fuhr, R. (1999): Praxisprinzipien: Gestalttherapie als experientieller, existentieller und experimenteller Ansatz. In: R. Fuhr, M. Sreckovic \& M. Gremmler Fuhr(Hrsg.): Handbuch der Gestalttherapie. S. 417-437. Göttingen : Hogrefe.

Fuhr, R., Sreckovic, M. \& Gremmler-Fuhr, M. (Hrag.) (1999): Handbuch der Gestaltherapie. Göttingen: Hogrefe.

Geiser, F., Imbierowicz, K., Schilling, G., Conrad, R. \& Liedtke, R. (2000): Unterschiede zwischen Diagnosegruppen psychosomatischer Patienten in der SymptomChecklist-90-R(SCL-90-R). Psychotherapie, Psychosomatik und medizinische Psychologie, 50,447-453.

Geisheim, C., Hahlweg, K., Fiegenbaum, W., Frank, M., Schröder, B. \& von Witzleben, I. (2002): Das Brief Symptom Inventory(BSI) als Instrument zur Qualitätssicherung in der Psychotherapie. Diagnostica, 48/1, 28-36.

Geller, B. \& Luby, J. (1997): Child and adolescent bipolar disorder. A view of the past 10 years. Journal of the American Academy oh Child and Adolescence Psychiatry, 36, 1168-1176.

Geller, B., Zimmernan, B., Williams, M., Bolhofner, K. \& Craney, J.L. (2001): Adult psychological outcome of prepubertal major depressive disorder. Journal of the American Academy of Child and Adolescence Psychiatry, 40, 673-677.

Goldberg, F.S., McNiel, D.E. \& Binder, R.L. (1988): Therapeutic Factors in two Forms of Inpatient Group Psychotherapy. Group, 12/3, 145-156.

Goldstein, K. (1934): Der Aufbau des Organismus: Einführung in die Biologie unter besonderer Berücksichtigung der Erfahrungen am kranken Menschen. Den Haag: Nidjhof.

Goodman, P. (1977): Nature Heals, In: T. Stohr (Eds.): The psychological Essays of Paul Goodman, New York; EP Duttons. dtsch. Natur heilt (1988). Köln: EHP.

Grawe, K. (1995): Grundriss einer Allgemeinen Psychotherapie. Psychotherapeut, 40, 130-145.

Grawe, K. (1997b): Research-informed psychotheray. Psychotherapy Research, 7, 1-20.

Grawe, K. (1999): Ressourcenaktivierung: Ein primöres Wirkprinzip der Psychotherapie. Psychotherapeut, 44, 63-73.

Grawe, K., Donati,R. \& Bernauer, F. (1994): Psychotherapie im Wandel - Von der Konfession zur Profession. 3. Aufl. Göttingen: Hogrefe.

Greenberg, L.S. (1979): Resolving splits: The Two-chair technique. Psychotherapy: Theory, Research and Practice, 16, 310-318.

Greenberg, L.S. (1984): A task Analysis of Intrapersonal Conflict Resolution. In: J.N. Rice \& L.S. Greenberg (Eds.): Patterns of Change-Intensive Analysis of Psychotherapy Process. New York: Guilford. 


\section{Literaturverzeichnis}

Greenberg, L.S. (2001): Emotion-Focused Therapy: Coaching Clients to Work Through Their Feelings. APA. dtsch. (2006): Emotionsfokussierte Therapie. Tübingen: dgvtVerlag.

Greenberg, L.S \& Higgins, H.M. (1980): Effects of Two-Chair Dialogue and Focusing on Conflict Resolution. Journal of Counseling Psychology, 27/3, 221-224.

Greenberg, L.S. \& Clarke, K. (1979): The differential effects of two-chair experiment and empathic reflections at a conflict marker. Journal of Counseling Psychology, 26, S. $1-8$.

Greenberg, L.S. \& Dompierre, L.M. (1981): Specific Effects of Gestalt Two-Chair Dialogue on Intrapsychic Conflict in Counseling. Journal of Counseling Psychology, 28/4, 288-294.

Greenberg, L.S. \& Foerster, F.S. (1996): Task Analysis Exemplified: The Process of Resolving Unfinished Business. Journal of Consulting and Clinical Psychology, 64/3, 439-446.

Greenberg, L.S. \& Malcolm, W. (2002): Resolving Unfinished Business: Relating Process to Outcome. Journal of Consulting and Clinical Psychology, 70/2, 406-416.

Greenberg, L.S. \& Paivio, S.C. (1997): Varieties of Shame Experience in Psychotherapy. Gesstalt Review, 3, 205-220.

Greenberg, L.S. \& Paivio, S.C. (1997): Working with emotions in psychotherapy. New York: Guilford.

Greenberg, L.S. \& Pinsof, W.M. (1986): The Psychotherapeutic Process. New York: Guilford.

Greenberg, L.S. \& Safran, J. (1989): Emotion in Psychotherapy, American Psychologist, 44/1, 19-29.

Greenberg, L.S. \& Safran, J.D. (1987): Emotion in psychotherapy. New York: Guilford.

Greenberg, L.S. \& Watson, J. (1999): Forschung zur gestalttherapeutischen Behandlung von Depressionen. In: R. Fuhr, M. Sreckovic \& M. Gremmler-Fuhr (Hrsg.): Handbuch der Gestalttherapie. S. 1121-1136. Göttingen: Hogrefe.

Greenberg, L.S. \& Watson, J. (1998): Experiential Therapy of Depression: Differential Effects of Client-Centered Relationship Conditions and Process Experiential Interventions. Psychotherapy Research, 8/2, 210-224.

Greenberg, L.S. \& Webster, M.C. (1982): Resolving Decisional Conflict by Gestalt TwoChair Dialogue: Relating Process to Outcome. Journal of Counseling Psychology, 29/5, 468-477.

Greenberg, L.S., Rice, L. \& Elliot, R. (1993): Facilitating emotional change; The Moment-byMoment Process. New York: Guilford. dtsch. Emotiale Veränderung fördern (2003): 


\section{Literaturverzeichnis}

Paderborn: Junfermann.

Gremmler-Fuhr, M. (2001): Grundkonzepte und Modelle der Gestalttherapie. In: R. Fuhr, M. Sreckovic \& M. Gremmler-Fuhr(Hrsg.): Handbuch der Gestalttherapie. 345-392 Göttingen: Hogrefe.

Grimm, L.G. \& Yarnold, P.R. (Hrsg.): Reading and understanding more multivariate statistics. Washington: American Psychological Association.

Groen, G. \& Petermann, F. (2002): Depressive Kinder und Jugendliche. Göttingen: Hogrefe

Groen, G. (2002): Der Verlauf depressiver Störungen im Jugendalter: Ergebnisse einer prospektiven Längsschnittstudie. Norderstedt: BoD.

Groen, G., Pössel, P., Al-Wiswasi \& Petermann, F. (2003): Universelle, schulbasierte Prävention der Depression im Jugendalter: Ergebnisse einer Follow-Up-Studie. Kindheit und Entwicklung, 12/3, 164-174.

Groen, G., Scheithauer, H., Essau, C.A. \& Petermann, F. (1997): Epidemologie depressiver Störungen in Kindes- und Jugendalter: Eine kritische Übersicht. Zeitschrift für Klinische Psychologie, Psychiatrie und Psychotherapie, 45, 115-144.

Gunkel, S. \& Kruse, G. (Hrsg.) (2004): Salutogenese, Resilienz und Psychotherapie. Hannover: Hannoversche Ärzte-Verlags-Union.

Hamilton, M. (1999): Depression: a Gestalt Therapy Perspektive. Psychotherapy in Australia, 5/3, 62-66.

Hampe, R., Ritschl, D. \& Waser, G. (Hrsg.) (1999): Kunst, Gestaltung und Therapie mit Kindern und Jugendlichen. Bremen: Verlag der Universität Bremen.

Hankin, B.L., Abramson, L.Y., Moffitt, T.E., Silvia, P.A., McGee, R. \& Angell, K.E. (1998): Development of depression from preadolescence to young adulthood: Emerging gender differences in a 10-years longitudinal study. Journal of Abnormal Psychology, 107, 128-140.

Harman, R.L. (Hrsg.) (1990): Gestalt Therapy Discussions with the Masters, Springfield: Charles C Thomas. dtsch. Werkstattgespräche Gestalttherapie(2001). Wuppertal: Peter Hammer.

Hartmann-Kottek, L. (2003): Gestalttherapie. Berlin: Springer.

Hausmann, B. \& Neddermeyer, R. (1996): Bewegt Sein. Paderborn: Junfermann.

Hautzinger, M. \& Petermann, F. (2003): Depression im Kindes- und Jugendalter: Einführung in den Themenschwerpunkt. Kinheit und Entwicklung, 12/3, 127-132.

Herpetz-Dahlmann, B. (1993): Essstörungen und Depression in der Adoleszenz. Göttingen : Hogrefe.

Hoffmann-Widhalm, H. (1999): Anwendung gestalttherapeutischer Grundprinzipien und Techniken in der Behandlung des posttraumatischen Syndroms am Beispiel 


\section{Literaturverzeichnis}

der Arbeit mit bosnischen Flüchtlingen. In: R. Hutterer-Kirsch, I. Luif \& G. Baumgartner(Hrsg.): Neue Entwicklungen in der Integrativen Gestalttherapie. 195-214. Wien: Facultas.

Höhmann-Kost, A. (2003): Materialmedien in der Integrativen Supervision. Supervision: www.fpi-publikationen.de/supervosion

Huf, A. (1992): Psychotherapeutische Wirfaktoren. Weinheim: Psychologie Verlag Union. Hutterer-Krisch, R. (1999): Zum Stellenwert der Diagnostik in der Integrativen Gestalttherapie. In: R. Hutterer-Kirsch, I. Luif \& G. Baumgartner (Hrsg.): Neue Entwicklungen in der Integrativen Gestalttherapie. S. 22-59. Wien: Facultas.

Hutterer-Krisch, R., Luif, I. \& Baumgartner, G. (Hrsg.) (1999): Neue Entwicklungen in der Integrativen Gestaltherapie. Wien: Facultas.

Hycner, R. (2003): Die Ich-Du-Beziehung. In: E. Doubrawa \& F.M. Staemmler (Hrsg.): Heilende Beziehung: Dialogische Gestaltherapie. 2. erweiterte Auflage. S. 83-94. Wuppertal: Peter Hammer Verlag.

Hycner, R. (2003): Für eine dialogische Gestalttherapie. In: E. Doubrawa \& F.M. Staemmler(Hrsg.): Heilende Beziehung : Dialogische Gestalttherapie. 2. erweiterte Auflage. S. 59-82. Wuppertal: Peter Hammer Verlag.

Hycner, R., Polster, E. \& Polster, M. (2003): Die dialogische Dimension der Gestalttherapie: Ein Gespräch. In: E. Doubrawa \& F.M. Staemmler (Hrsg.): Heilende Beziehung : Dialogische Gestaltherapie. 2. erweiterte Auflage. S. 203-230. Wuppertal: Peter Hammer Verlag.

Jacobs, L. (2003): Ich und Du, hier und jetzt. : Zur Theorie und Praxis des Dialogs in der Gestalttherapie. In: E. Doubrawa \& F.M. Staemmler (Hrsg.): Heilende Beziehung : Dialogische Gestaltherapie. 2. erweiterte Auflage. S. 95-124. Wuppertal: Peter Hammer Verlag.

Johnson, S.M. \& Greenberg, L.S. (1985): Differential Effects of Experiential and Problem-Solving Interventions in Resolving Marital Conflict. Journal of Consulting and Clinical Psychology, 53/2, 175-184.

Junge, J., Neumer, S., Manz, R. \& Margraf, J. (2002): Go!- Gesundheit und Optimismus:

Ein Trainingsprogramm gegen Angst und Depression für Jugendliche. Weinheim: Psychologie Verlags Union.

Kashani, J.H. \& Carlson, G.A. (1987): Seriously depressed preschoolers. American Journal of Psychiatry, 144, 348-350.

Kellam, S.G., Rebok, G.W., Mayer, L.S., Ialongo, N. \& Kalodner, C.R. (1994): Depressive symptoms over first grade and their response to a developmental epidemiologically based preventive trial aimed at improving achievement. Development and 


\section{Literaturverzeichnis}

Psychopathology, 6, 463-481.

Kemmler, L., Schelp, T. \& Mecheril, P. (1991): Sprachgebrauch in der Psychotherapie. Göttingen: Hans Huber.

Kepner, J.I Greenberg, L.S. (1987): Body Process: A Gestalt Approach to Working with the

Body in Psychotherapy. New York: Gardner. dtsch. Körperprozess(1989). Köln: EHP.

Kerns, L.L. \& Liebeman, A.B. (1996): Hilfen für depressive Kinder, Bern: Huber.

Kim, J.K. (1995): Gestalt Psychotherapy. Seoul: Hakjisa.

Kim, J.K. (2003): A Gestalt Therapy approach to Anxiety disorders integrating Mindfulness meditation and Cognitive Behavioral Therapy, The Korean Journal of Clinical Psychology, 22/3, 475-503.

Kim, M. S., Kim, H. W. \& Cha, K. H. (2001): Analyses on the Construct of Psychological Well-Being (PWB) of Korean Male und Female Adults. Korean Journal of Social and Personality Psychology, 15/2, 19-39.

Kivlighan, D.M., Multon, K.D. \& Brossart, D.F. (1996): Helpful Impacts in Group Counseling: Develpoment of a Multidimensional Rating System. Journal of Counseling Psychology, 43/3, 347-355.

Klein, D.N., Lewinsohn, P.M. \& Seeley, J.R. (1997): Psychological characteristics of adolescents with a past history of dysthymic disorder: Comparison with adolescents with past histories of major depressive and non affective disorders, and never mantally ill controls. Journal of Affective Disorders, 42, 127-135.

Koffka, K. (2001): Das Verhalten und sein Feld: Die Aufgabe der Psychologie-Teil1. Gestalt Theory, 23/2, 85-101.

Koffka, K. (2001): Das Verhalten und sein Feld: Die Aufgabe der Psychologie-Teil2. Gestalt Theory, 23/4, 239-266.

Köhler, W. (1955): Direction of processes in living systems. Scientific Monthly, 80, 29-32.

Korb, M.P., Gorrell, J. \& van de Riet, V. (1989): Gestalt Therapy: Practice and Theory, 2. Ed. New York: Pergamon.

Kosfelder, J., Michalak, J., Vocks, S. \& Willutzki, U. (Hrsg.) (2005): Fortschritte der Psychotherapieforschung. Göttingen: Hogrefe.

Kovacs, M., Feinberg, T.L., Crouse-Novak, M.A., Paulauskas, S.L. \& Finkelstein, R. (1984b): Depressive disorders in childhood: I . A longitudinal prospective study of characteristics and recovery. Archives of General Psychiatry, 41, 229-237.

Kovacs, M., Gatsonis, C., Paulaskas, S.L. \& Richards, C. (1989): Depressive Disorders in childhood. IV. A longitudinal study of comorbidity with and risk for anxiety disorders. Archieves of General Psychiatry, 46, 776-782.

Krause, C. (2003): Gesundheitsberatung. In: C. Krause, B. Fittkau, R. Fuhr \& H.U. Thiel 


\section{Literaturverzeichnis}

(Hrsg.), Pädagogische Beratung. S. 187-204. Pardeborn: Ferdinand Schöningh.

Krause, C. (2003): Pädagogische Beratung: Was ist, was soll, was kann Beratung. In: C.

Krause, B. Fittkau, R. Fuhr \& H.U. Thiel (Hrsg.), Pädagogische Beratung. S. 15-31.

Pardeborn: Ferdinand Schöningh.

Krisch, R.(1992): Der Krnakheitsbegriff in der Gestalttherapie. In: A. Pritz \& H.G.Petzold(Hrsg.): Der Krankheitsbegriff in der modernen Psychotherapie. S. 197252, Paderborn: Junfermann.

Krüger, R.T. (2004): Schöpferisch sein- wieder schöpferisch werden. Kreativität als Ressource des Menschen und als Mittel der Heilung in der Psychotherapie. In: S. Gunkel \& G. Kruse (Hrsg.): Salutogenese, Resilienz und Psychotherapie. S. 257-294. Hannover: Hannoversche Ärzte-Verlags-Union.

Kurstjens, S. \& Wolke, D. (2001): Effects of maternal depression on cognitive development of children over the first 7 years of life. Journal of Psychology und Psychiatry, 42, 623-636.

Laireiter, A.R. (2000): Diagnostik in der Psychotherapie. Wien, NewYork: Springer.

Leedy, J.J. (1985): Prinzipien der Poesietherapie. In : H.G. Petzold \& I. Orth (Hrsg.): Poesie und Therapie : Über die Heilkraft der Sprache. S. 243-248. Paderborn: Junfermann Verlag.

Lewin, K. (1963): Feldtheorie in den Sozialwissenschaften. Ausgewählte theoretische Schriften. Berlin: Huber.

Lewinsohn, P.M., Hops, H., Roberts, R.E. Seeley, J.R. \& Andrews, J.A. (1993): Adolescent psychopathology: I . Prevalence and incidence of depression and other DSM-III-R disorders in high school students. Journal of Abnormal Psychology, 102, 133-144.

Lewinsohn, P.M., Rohde, P. Seeley, J.R. (1998a): Major depressive disorder in older adolescents: Prevalence, risk factors, and clinical implications. Clinical Psychology Review, 18, 765-794.

Lewinsohn, P.M., Rohde, P., Klein, D.N. \& Seeley, J.R. (1999): Natural course of adolescent major depressive disorder: I . Continuity into young adulthood. Journal of the American Academy of Child and Adolescent Psychiatry, 38, 56-63.

Lumma, K. \& Knüdeler, B. (1984): Medien für kreative Gestalt- Arbeit. Lumma + Kern.

Märtens, M. \& Petzold, H.G (1995): Psychotherapieforschung und kinderpsychotherapeutische Praxis. Praxis der Kinderpsychologie und Kinderpsychiatrie. 44, 302-321.

McCauley, E., Myers, K., Mitchell, J., Calderon, R., Schloredt, K. \& Treder, R. (1993): Depression in young people: Initial presentation and clinical course. Journal of the 


\section{Literaturverzeichnis}

American Academy of Child and Adolescent Psychiatry, 32, 714-722.

McGee, R., Feehan, M., Williams, S. \& Anderson, J. (1992): DSM-III disorders from age 11 to 15 years. Journal of the American Academy of Child and Adolescence Psychiatry, 31, 50-59.

Melnick, J. \& Nevis, S.M. (1997): Diagnosing in the here and now: The experience Cycle and DSM-IV. British Gestalt Journal, 6/2, 97-106.

Metzger, W. (1962): Schöpferische Freiheit.Frankfurt/M: Waldemar Kramer.

Miller, M.V. (2006): Unser Verhältnis zur Welt gestalten und verwandeln: Über Kunstwerke und Symptome. Gestaltkritik.

Onlinequelle: http://www.gestalt.de/miller_kunstwerke.html .

Miller, N. (2006): Bringing intuitive creative skills to work environments. Gestalt. 9/2. in Online http://www.g-gej.org/9-2/creativework.html.

Missirlian, T.M., Toukmanian, S.G., Warwar, S.H. \& Greenberg, L.S. (2005): Emotional Arousal, Client Perceptual Processing, and the Working Alliance in Experiential Psychotherapy for Depression, Journal of Consulting and Clinical Psychotherapy, 73/5, 861-871.

Müller, B. (1988): Zur Theorie der Diagnostik narzißtischer Erlebens- und Verhaltensstrukturen. Gestalttherapie, 2, 43-58.

Müller, B. (1999): Ein kategoriales Modell gestalttherapeutischer Diagnostik. In: R. Fuhr, M. Sreckovic \& M. Gremmler-Fuhr (Hrag.): Handbuch der Gestalttherapie. S. 647-671. Göttingen: Hogrefe.

Müller, L. \& Petzold, H.G. (1998): Projektive und semiprojektive Verfahren, „kreative und virtuelle Medien“ für die Diagnostik von Störungen von sozialen Netzwerken und Komorbidität in der Integrativen Therapie mit Kindern und Jungendlichen. Integrative Therapie, 3-4, 396-437.

Nitsch-Berg, H. \& Kühn, H. (2000): Kreative Medien und die Suche nach Identität. Bd.1,2. Köln: Edition Humanistische Psychologie.

Nußbeck, S. (2006): Beratung Psychologie. München: Ernst Reinhardt.

Oaklander, V. (1978): Windows to Our Children. Moab: Real People Press. dtsch: (1981). Gestalttherapie mit Kindern und Jugendlichen. Stuttgart: Klett-Cotta.

Oaklander, V. (1979): A gestalt therapy approach with children through the use of art and creative expression. In: E.H. Marcus(Eds.): Gestalt therapy and beyond, 235-247. CA: Meta Cupertino.

Olges, B. M., Lambert, M. J. \& Masters, K. S. (Eds.) (1996): Assessing outcome in clinical practice. Needham Heights, MA: Allyn \& Bacon.

Orth, I. (1995): Unbewusstes in der therapeutischen Arbeit mit künstlerischen 


\section{Literaturverzeichnis}

Methoden, kreativen Medien. Integarative Therapie, 1. 321-339.

Orth, I. (2002): Weibliche Identität und Leiblichkeit- Prozesse „konvivialer“ Veränderung und Entwicklung: Überlegung für die Praxis. Integrative Therapie, 4 .

Orth, I. \& Petzold, H.G. (1990): Metamorphosen- Prozess der Wandlung in der intermedialen Arbeit der Integrativen Therapie. In: H.G.Petzold \& I.Orth(Hrsg.): Die neuen Kreativitätstherapien. Bd.2. S. 721-774. Paderborn: Junfermann Verlag.

Orth, I. \& Petzold, H.G. (1993): Zur Anthropologie des schöpferischen Menschen. In: H.G. Petzold \& Sieper, J. (Hrsg.): Integration und Kreation. Bd.1. S. 93-116. Paderborn: Junfermann Verlag.

Paivio, S. \& Greenberg, L.S.(1995): Resolving "Unfinished Business": Efficacy of Experiential Therapy Using Empty-Chair Dialogue. Journal of Consulting and Clinical Psychology, 63/3, 419-425.

Paivio, S. \& Greenberg, L.S. (1995): Resolving unfinished business. Experiential therapy using empty chair dialogue. Journal of Consulting and Clinical Psychology, 63, 419-425.

Parlett, M. (1994): Variationen im therapeutischen Dialog, In: Freiler, C., VentouratouSchmetterer, D., Reiner-Lawugger, C. \& Bösel, R. (Hrsg.): 100 Jahre Fritz Perls: Tagungsband der Internationalen Psychotherapietagung der Fachsektion für Integrative Gstalttherapie ÖAGG. S. 229-232. Facultas: Wien

Parlett, M. (1994): Wir erschaffen unsere Systeme, unsere Systeme erschaffen uns. Feldtheorie für Gestalttherapeuten. In: C. Freiler, D. Ventouratou-Schmetterer, C. Reiner-Lawugger \& R. Bösel (Hrsg.): 100 Jahre Fritz Perls: Tagungsband der internationalen Psychotherapietagung der Fachsektion für Integrative Gestalttherapie ÖAGG. S. 119-130. Wien: Facultas-Universitätsverlag.

Parlett, M. (2003): Schöpferische Fähigkeiten und die Lebenskunst. In: M. Spagnuolo Lobb \& N. Amendt-Lyon. (Hrsg.): Creative License: The Art of Gestalt Therapy. New York: Springer. dtsch. (2006): Die Kunst der Gestalttherapie: eine schöpferische Wechselbeziehung. S. 61-76. Wien: Springer.

Pauls, H. \& Reicherts, M. (1999): Empirische Forschung in der Gestalttherapie am Beispiel eines praxisorientierten Forschungsprojektes. In: R. Fuhr, M. Sreckovic \& M. Gremmler-Fuhr (Hrag.): Handbuch der Gestalttherapie. S. 1137-1160. Göttingen: Hogrefe.

Perls, F. S. (1947): Ego, hunger, and aggression. London: Allen \& Unwin. dtsch: (1978) Das Ich, der Hunger und die Aggression. Stuttgart: Klett-Cotta.

Perls, F. S. (1966): Gestalttherapie und die menschliche Potentiale. In: H.G. Petzold (Hrsg.) (1980): Gestalt, Wachstum, Integration. Parderborn: Junfermann Verlag. 


\section{Literaturverzeichnis}

Perls, F. S. (1969): Gestalt Therapy Verbatim. Lafayette: Real People Press. dtsch.: GestaltTherapie in Aktion. (1979). 3. Aufl. Stuttgart: Klett-Cotta.

Perls, F. S. (1970): Four Lectures. In J. Fagan \& I. Shepherd (Eds.): Gestalt therapy now. S. 14-38. New York: Harper.

Perls, F. S., Hefferine. R.F. \& Goodman, P. (1951): Excitment and Growth in the Human Personality. dtsch. Gestaltherapie. Lebensfreude und Persönlichkeitsentfaltung (1979) Stuttgart: Klett-Cotta.

Perls, F. S., Hefferine. R.F. \& Goodman, P. (1951): Excitment and Growth in the Human Personality. dtsch. Gestaltherapie.Wiederbelebunh des Selbst (1979). Stuttgart: KlettCotta.

Perls, L. (1989): Leben an der Grenze. Köln: EHP.

Perls, L. (2005): Der Therapeut ist ein Künstler, Gestaltkritik, 1.

Petersen, A.C., Leffert, N., Graham, B., Alwin, J. \& Ding, S. (1997): Promoting mental health during the transition into adolescence. In: J.Schilenberg, J.L. Maggs \& Hurrelmann(Eds.): Health risks and developmental transitions during adolescence. S. 471497. Cambrige: Cambrige Univeristy Press.

Petitjean, S. (2002): Wirksamkeit im Psychotherapieverfahren der Integrativen Therapie. Graduierungsarbeit am FPI.

Petzold, H.G. (Hrsg.) (1980): Gestalt, Wachstum, Integration. Parderborn: Junfermann Verlag.

Petzold, H.G.(Hrsg.)(1985): Wege zum Menschen. Bde.2. Parderborn: Junfermann

Petzold, H.G (1987a): Puppen und Puppenspiel in der Integrativen Therapie mit Kindern, In: H.G. Petzold \& G. Ramin (Hrsg.): Schulen der Kinderpsychotherapie, S. 427-490. Paderborn: Junfermann Verlag.

Petzold, H.G. (1988n): Integrative Bewegungs- und Leibtherapie: Ein ganzheitlicher Weg leibbezogener Psychotherapie, 2Bde. Paderborn: Junfermann Verlag.

Petzold, H.G. (1992): Grundzüge des Krankheitsbegriffes im Entwurf der Integrativen Therapie. In: A. Pritz \& H.G. Petzold (Hrsg.): Der Krankheitsbegriff in der modernen Psychotherapie. S. 371-486, Paderborn: Junfermann.

Petzold, H.G. (1997): Das Ressourcenkonzept in der sozialinterventiven Praxeologie und Systemberatung. Integrative Therapie, 4, 435-471.

Petzold, H.G. (1999b): Die Chance der Begegnung. In H.G. Petzold (2003): Integrative

Therapie. 2. Aufl. Bd.3. S. 781-808. Parderborn: Junfermann Verlag.

Petzold, H.G. (2003): Integrative Therapie. 2. Aufl. Bd.1,2,3. Parderborn: Junfermann Verlag.

Petzold, H. \& Sieper, J. (Hrag.) (1996): Integration und Kreation. 2Bde. Paderborn: 


\section{Literaturverzeichnis}

Junfermann.

Petzold, H.G \& Ramin, G (Hrsg.) (1987): Schulen der Kinderpsychotherapie. Paderborn: Junfermann Verlag.

Petzold, H.G. \& Orth, I (Hrsg.) (1990): Die neuen Kreativitätstherapien. 2 Bde. Paderborn: Junfermann Verlag.

Petzold, H.G. \& Orth, I. (1985): Poesie und Bibliotherapie: Entwicklung, Konzepte und

Theorie - Methodik und Praxis des Integrativen Ansatzes. In: H.G. Petzold \& I. Orth(Hrsg.): Poesie und Therapie: Über die Heilkraft der Sprache. S. 21-101. Paderborn: Junfermann Verlag.

Petzold, H.G. \& Orth, I. (Hrsg.) (1985): Poesie und Therapie: Über die Heilkraft der Sprache. Paderborn: Junfermann Verlag.

Polster, E. \& Polster, M. (1973): Gestalt Therapy Integrated. New York: Brunner/Matzel. dtsch. (1975). Gestalttherapie. München: Kindler Verlag.

Portele, G.H.(1992): Der Mensch ist kein Wägelchen. Köln: EHP.

Portele, H. (2003): Martin Buber für Gestalttherapeuten: Fritz und Laura Perls und Buber. In: E. Doubrawa \& F.M. Staemmler (Hrsg.): Heilende Beziehung : Dialogische Gestalttherapie. 2. erweiterte Auflage. S. 11-26. Wuppertal: Peter Hammer Verlag.

Pos, A.E. \& Greenberg, L.S. (2007): Emotion-focused Therapy: The Transforming Power of Affect. Journal of Contemporary Psychotherapy, 37, 25-31.

Pos, A.E., Greenberg, L.S., Goldman. R.N. \& Korman, L.M. (2003): Emotional Processing during experiential Treatment of Depression. Journal of Consulting and Clinical Psychology, 71/6, 1007-1016.

Powers, S.I., Hauser, S.T. \& Killner, L.A. (1989): Adolescent mental health. American Psychologist, 44, 200-208.

Pritz, A. \& Petzold, H.G. (Hrsg.) (1992): Der Krankheitsbegriff in der modernen Psychotherapie. Paderborn: Junfermann.

Rahm, D. (2000): Gestaltberatung: Grundlage und Praxis integrativer Beratungsarbeit. Paderborn: Junfermann Verlag.

Rahm, D. (1997): Integrative Gruppentherapie mit Kindern. Göttingen: Vandenhoeck \& Ruprecht.

Rao, U., Hammen, C. \& Daley, S. (1999): Continuity of depression during the transition to adulthood. A 5-year longitudinal study of young women. Journal of the American Academy of Child and Adolescence Psychiatry, 33, 908-915.

Rapp, E. (1980): Gestaltungstherapie in Gestaltgruppen. In: R. Ronall \& B. Feder (Eds.): Beyond of the Hot Seat. New York: Brunner/Mazel. dtsch. Gestaltgruppen.(1983). Stuttgart: Klett-Cotta. 


\section{Literaturverzeichnis}

Renouf, A. G. \& Harter, S. (1990): Low-self-worth and anger as components of the depressive experience in young adolescents. Development and Psychopathology, 2. 293-310.

Resnick, R.(1990). Interview with Robert Resnick. In: R. L. Harman. (Ed.): Gestalt Therapy Discussion with the masters. Springfield: Charles C Thomas. dtsch. (2001): Werkstattgespräche Gestalttherapie. S. 91-111. Wuppertal: Perter Hammer.

Reynolds, W.M. \& Johnston, H.F. (Eds.): Handbook of depression in children and adolescents. New York: Plenum

Rice, L.N. \& Greenberg, L.S. (Eds.) (1984): Patterns of Change-Intensive Analysis of Psychotherapy Process. New York: Guilford.

Richter, K.F. (2003): Erzähweisen des Körpers. 3. Aufl. Seelze-Velber: Kallmeyer.

Rogers, N.(2001): Person-Centered Expressive Arts Therapy. In: J.A. Rubin(Eds.): Approaches to Art Therapy (2.Ed.). S. 163-177. Philadelphia: Brunner-Routledge.

Rohde, P., Lewinsohn, P.M \& Seeley, J.R. (1991): Comorbidity of unipolar depression: II. Comorbidity with other mental disorders in adolescents and adults. Journal of Abnormal Psychology, 100, 214-222.

Rohde, P., Lewinsohn, P.M \& Seeley, J.R. (1994): Are adolescents changed by an episode of major depression?. Journal of the American Academy of Child and Adolescence Psychiatry, 33, 1289-1298.

Rojas-Bermúdez, J.B. (1983): Handpuppe als Intermediär-Objekte in der Behandlung von Psychotikern, In: H.G. Petzold (Hrsg.): Puppen und Puppenspiel in der Psychotherapie. S. 129-160. München : Pfeiffer

Ronall, R \& Feder, B. (Eds.) (1980): Beyond of the Hot Seat. New York: Brunner/Mazel. dtsch. Gestaltgruppen (1983): Stuttgart: Klett-Cotta.

Rosal, M. (2001): Cognitive- Behavioral Art Therapy. In: J.A.Rubin (Eds.): Approaches to Art Therapy (2.Ed.). 210-225. Philadelphia: Brunner-Routledge.

Rosenfeld, E. (1978): An Oral History of Gestalt Therapy. Part 1: An Conversation with Laura Perls. The Gestalt Journal 1/1. dtsch. Aus dem Schatten treten. Gestaltkritik, 2/2001.

Rosner, R. (1996): The relationship between emotional expression, treatment, and outcome in psychotherapy. Frankfurt: Lang.

Rosner, R., Frick, U., Beutler, L.E. \& Daldrup, R. (1999): Depressionsverläufe in unterschiedlichen Psychotherapieformen: Modellierung durch Hierarchische Lineare Modelle(HLM). Zeitschrift für klinische Psychologie, 28/2, 112-120.

Rossmann, B. (2001): Depressive Strörungen. In: G. Esser (Hrsg.): Lehrbuch der klinischen Psychologie und Psychotherapie des Kinde und Jugendalters. S. 263-276. Stuttgart: Georg 


\section{Literaturverzeichnis}

Thieme.

Rubin, J.A. (Eds.) (2001): Approaches to Art Therapy (2.Ed.). Philadelphia: BrunnerRoutledge.

Rude, S.S. \& Bates, D. (2005): The Use of Cognitive and Experiential Techniques to Treat Depression. Clinical Case Studies, 4, 363-379.

Rudolph, K.R., Hammen, C. \& Burge, D. (1994): Interpersonal functioning and depressive symptoms in childhood: Addressing the issues of specificity and comorbidity. Journal of Abnormal Child Psychology, 22, 355-371.

Rumpler, P. (2007): Freud aus der Sicht eines Gestalttherapeuten: Des einen Freud'....des andern Leid. Integrative Therapie, 33, 1/2, 87-100.

Rutter, M. (1986): The developmental psychopathology of depression: Issues and perspectives. In: Rutter, M, Izard, C.E., Read, P.B. (Eds.): Depression in young people. New York: The Guilford Press, 3-30.

Ryff, C. D. (1989a): Beyond Ponce de Leon and Satisfaction: New directions in quest of successful aging. International Journal of Behavioral Development, 12, 35-55.

Ryff, C. D. (1989b): In the eye of the beholder: Views of psychological well-being among middle and old aged adults. Psychology and Aging, 4, 195-210.

Ryff, C. D (1989c): Happiness Is Everything, or Is It? Explorations on the Meaning of Psychological Well-Being. Journal of Personality and Social Psychology, 57/6, 10691081

Ryff, C. D. \& Keyes, CL.M. (1995): The Structure of Psychological Well-Being Revisited. Journal of Personality and Social Psychology, 69/4, 719-727.

Schmutte, P. S. \& Ryff, C. D. (1997): Personality and Well-Being: Reexaming Methods and Meanings. Journal of Personality and Social Psychology, 73/3, 549-559.

Schneider, K. (1979). Das Experiment in der Gestalttherapie, In: Integrative Therapie, 3. 192-207.

Schochet, I.M., Dadds, M., Holland, D., Whitefield, K., Harnett, P.H. \& Osgarby, S.M. (2001): The efficacy of a universal school-based program to prevent adolescent depression. Journal of Clinical Child Psychology, 30, 303-315.

Schönig.W. \& Brunner, E.J. (1990): Beratung in pädagogoschen, sozialpädagogischen und psychologischen Praxisfeldern. In: W.J. Brunner \& W. Schönig. Theorie und Praxis von Beratung: pädagogische und psychologische Konzepte. 7-27, Freiburg in Breisgau: Lambertus.

Schuch, B. (2000): Psychodiagnostik in der Psychotherapie von Kindern und Jugendlichen. In. A.R. Laireiter (Hrsg.): Diagnostik in der Psychotherapie. 405-419. Wien, NewYork: Springer. 


\section{Literaturverzeichnis}

Schulte-Markwort, m. \& Bindt, C. (2006): Psychotherapie im Kindes- und Jugendalter. Psychotherapeut, 51, 72-79.

Scönig, W. (1990): Wer sind die Adressaten einer Beratung in der Schule? In: W.J. Brunner \& W. Schönig. Theorie und Praxis von Beratung: pädagogische und psychologische Konzepte. 111-128, Freiburg in Breisgau: Lambertus.

Seiffge-Krenke, I. \& Klessinger, N. (2001): Gibt es geschlechtsspezifische Faktoren in der Vorhersage depressiver Symptome im Jugendalter?. Zeitschrift für Klinische Psychologie und Psychotherapie, 30/1, 22-32.

Shaw, J. A (1988): Childhood depression. Medical Clinics of North America, 72, 831-845.

Spagnuolo Lobb, M. \& Amendt-Lyon, N. (Hrsg.) (2003): Creative License: The Art of Gestalt Therapy. New York: Springer. dtsch. (2006): Die Kunst der Gestalttherapie: eine schöpferische Wechselbeziehung. Wien: Springer.

Stein, R. (2005): Einführung in die pädagogische Gestaltarbeit. Baltmannsweiler: Schneider VerlagHohengehren.

Steinhausen, H.C. (1993): Psychische Störungen bei Kindern und Jugendlichen. 2.Aufl. München, Wien, Baltimore: Urban \& Schwarzenberg.

Stevens, J.O. (1971): Awareness: exploring, experimenting, experiencing. Moab: Real People. dtsch. Die Kunst der Wahrnehmung(1975). München: Chr. Kaiser.

Strümpfel, U. (2004): Arbeit mit Gefühlen in der prozess-erlebensorientierten Therapie nach Greenberg, Rice \& Elliot. Integrative Therapie, 4, 363-375.

Strümpfel, U. (2006): Therapie der Gefühle: Forschungsbefunde zur Gestalttherapie. Bergisch Gladbach: Edition Humanistische Psychologie.

Strupp, H. H., Horowitz, L. M. \& Lambert, M.J. (Eds.) (1997): Measuring patient change in mood, anxiety, and personality disorders. Toward a core battery. Washington, DC: APA.

Swanson, C. \& Lichtenberg, P. (1998): Diagnosis in Gestalt Therapy: A Modest Beginning. The Gestalt Journal, 21/1, 5-17.

Teschke, D. (1996): Existentielle Momente in der Psychotherapie: Eine empirische Untersuchung mit gestalttherapeutischer Perspektive. Münster: LIT.

Teschke, D. (1999): Gestalttherapeutische Prozessforschung am Beispiel einer Untersuchung über Existentielle Momente in der Psychotherapie. In: R. Fuhr, M. Sreckovic \& M. Gremmler-Fuhr (Hrag.): Handbuch der Gestalttherapie. 1161-1178. Göttingen: Hogrefe.

Thomas, R. (1999): Der Begriff der Gestalt in der Musik und der Psychologie. In: I. Frohne-Hagemann(Hrsg.): Musik und Gestalt, 13-35. Göttingen: Vandenhoeck \& Ruprecht. 


\section{Literaturverzeichnis}

Tophoff, M. (1994): Gestalttheorie als ästhetisches Veränderungsparadigma. In: C. Freiler, D. Ventouratou-Schmetterer, C. Reiner-Lawugger \& R. Bösel (Hrsg.): 100 Jahre Fritz Perls: Tagungsband der internationalen Psychotherapietagung der Fachsektion für Integrative Gestalttherapie ÖAGG. S. 111-118. Wien: FacultasUniversitätsverlag.

Tschuschke, V. (1993): Wirkfaktoren stationärer Gruppenpsychotherapie. Göttingen: Vandenhoeck \& Ruprecht.

Tyson, G.M. \& Range, L.M. (1987): Gestalt Dialogues as a Treatment for mild Depression: Time Work just as well. Journal of Clinical Psychology, 43/2, 227-231.

Vocks, S., Joormann, J., Michalak, J., Kosfelder, J. \& Meyer, F. (2005): Diagnostik und Erfolgsmessung. In Kosfelder, J., Michalak, J., Vocks, S. \& Willutzki, U. (Hrsg.): Fortschritte der Psychotherapieforschung. 89-112, Göttingen: Hogrefe.

Votsmeier, A. (1994): Die Gestalttherapie als ganzheitlich-feldtheoretischer Ansatz: Ihre Methodologie in Verbindung mit den Wirkprinzipien von GRAWE. Eine Vefassungsmanuskript, Saabrücker Gestalt-Institut.

Wagner-Moore, L.E. (2004): Gestalt Therapy: Past, Present, Theory and Research. Psychotherapy: Theory, Research, Practice, Training, 41/2, 180-189.

Waldenfels, B. (1992): Einführung in die Phänomenologie. München: W. Fink.

Walter, H.J. (1994): Gestalttheorie und Psychotherapie. 3. Aufl. Opladen: Westdeutscher Verlag.

Weinfurt, K.P. (2000): Repeated measures analysis: ANOVA, MANOVA, HLM. In L.G. Grimm \& P.R. Yarnold (Hrsg.): Reading and understanding more multivariate statistics 317-362. Washington: American Psychological Association.

Weiss, B. \& Carton, T. (1994): Specificity of the comorbidity of aggression and depression in children. Journal of Abnormal Child Psychology, 22, 389-401.

Weymann, E. (1999): Kunstanaloges Vorgehen in der Musiktherapie. In: I. FrohneHagemann (Hrsg.): Musik und Gestalt, S. 48-70. Göttingen: Vandenhoeck \& Ruprecht.

Wiitchen, H.U., Knäuper, B. \& Kessler, R.C. (1994): Lifetime risk of depression. British Journal of Psychiatry, 165, 16-22.

Winicott, D.W. (1971): Playing and Reality, London: Tavistock. dtsch(1973): Vom Spiel zur Kreativität, Stuttgart: Klett-Cotta.

Wittchen, H.U., Essau, C.A., von Zerssen, D., Krieg, J.C. \& Zaudig, M. (1992): Lifetime and Six-Month Follow-Up Study, European Archives of Psychiatry and Clinical Neuroscience, 241, 247-258.

Wymore, J. (2006): Gestalt Therapy and Human Nature. Bloomington: Author House. 


\section{Literaturverzeichnis}

Yalom, I. (1995): The Theory and Practice of Group Psychotherapy. 4. Eds.New York: Basic. dtsch. (1996): Theorie und Praxis der Gruppenpsychotherapie. 4. Aufl. München: Pfeiffer.

Yontef, G. (1993): Awareness, dialogue and process : Essay in Gestalt therapy. Highland, New York: The Gestalt Journal Press. dtsch. (1999). Awareness, Dialog und Prozess. EHP.

Yontef, G. (1999): Preface to the German edition of "Awareness, dialogue and process." The Gestalt Journal, 22/1, 9-20.

Yontef, G. (2002): The Relational Attitude in Gestalt Therapy Theory and Practice International Gestalt Journal , 25/1, 15-35.

Yontef, G. (2003): Gestalttherapie als dialogische Methode. In: E. Doubrawa \& F.M. Staemmler (Hrsg.): Heilende Beziehung: Dialogische Gestalttherapie. 2. erweiterte Auflage. S. 27-58. Wuppertal: Peter Hammer Verlag.

Yontef, G. (2007): The power of the Immediate Moment in Gestalt Therapy. Journal of Contemparary Psychotherapy, 37, 17-23.

Yontef, G.M. \& Jacobs, L. (2000): Gestalt Therapy. In: R.J. Corsini \& D. Wedding(eds.): Current Psychotherapies, $6^{\text {th }}$ edition. Illinois: F.E. Peacock.

Zepf, S. \& Hartmann, S. (2002): Wissenschaftliche Prüfung und wisschenschaftliche Anerkennung psychotherapeutischer Verfahren: Einige grundsätzliche Anmerkungen $\mathrm{zu}$ den Prüfkriterien des wissenschaftlichen Beirats "Psychotherapie“. Psychotherapeut, 47/5, 278-284.

Zinker, J. (1977): Creative process in gestalt therapy. New York:Brunner/Mazel. dtsch. (1982).Gestalttherapie als kreativer Prozess. Paderborn: Junfermann Verlag. 
Anhang

Anhang 


\section{A. Darstellung der im Programm gestalteten Produkte von Teilnehmerinnen}

1. Sitzung: Selbstdarstellung

2. Sitzung: Familie in Tieren

3. Sitzung: Schulpanorama

4. Sitzung: Negative Gefühle zum Selbst

6.Sitzung: Unterbrochene Gefühle zu einer bestimmten Person

9. Sitzung: Ein Baum als I ch 


\section{Sitzung: Selbstvorstellung}
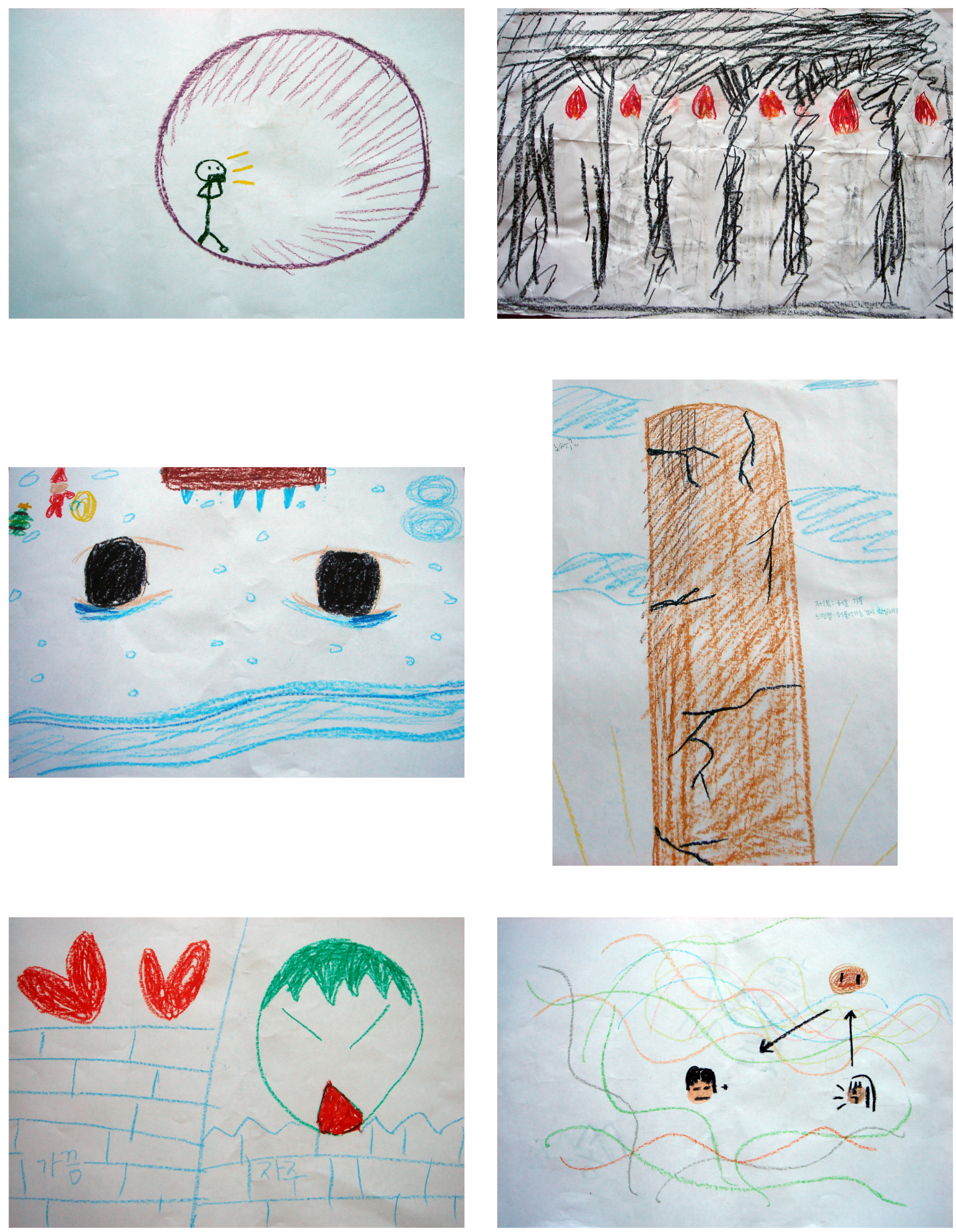


\section{Sitzung: Familie in Tieren}
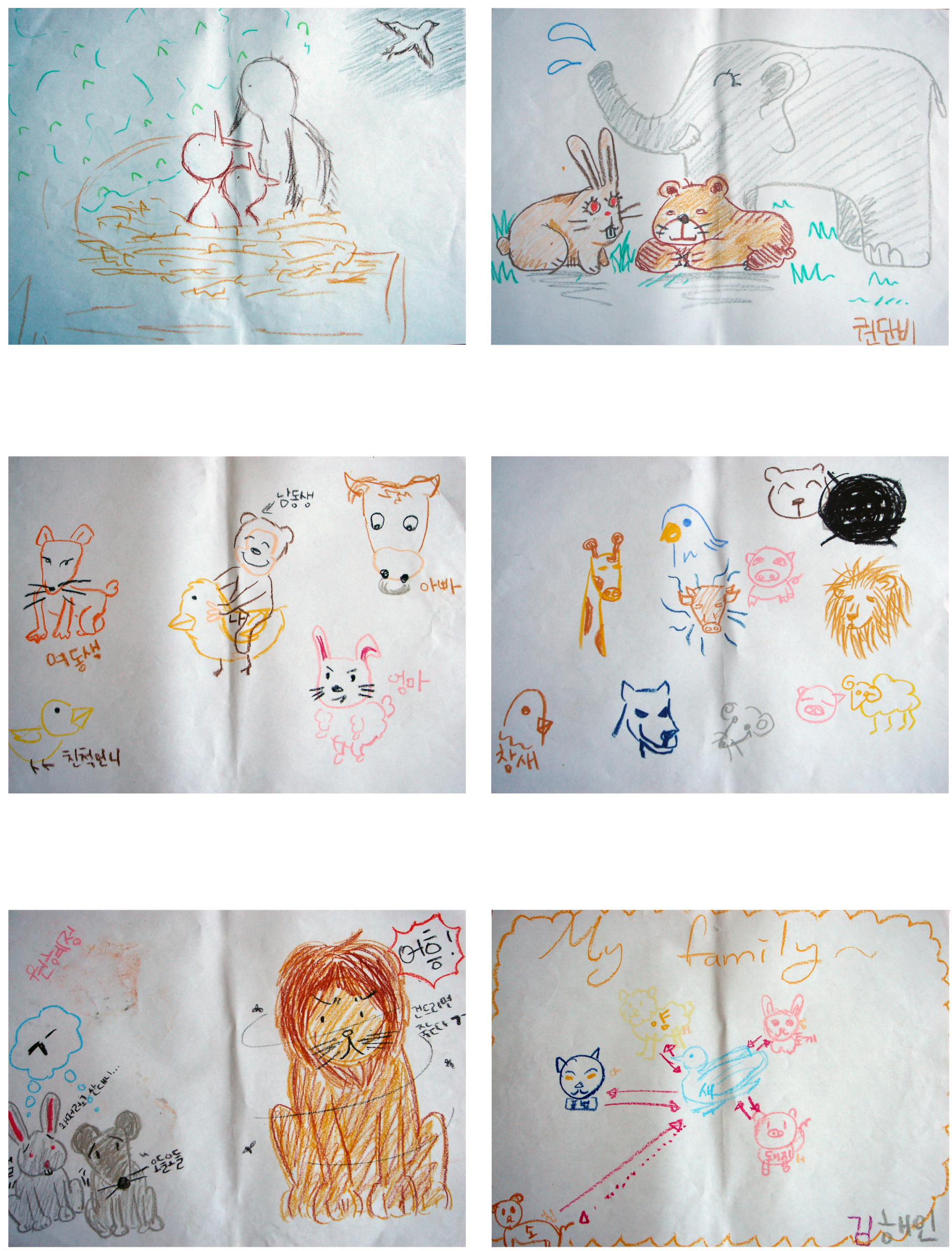


\section{Sitzung: Schulpanorama}
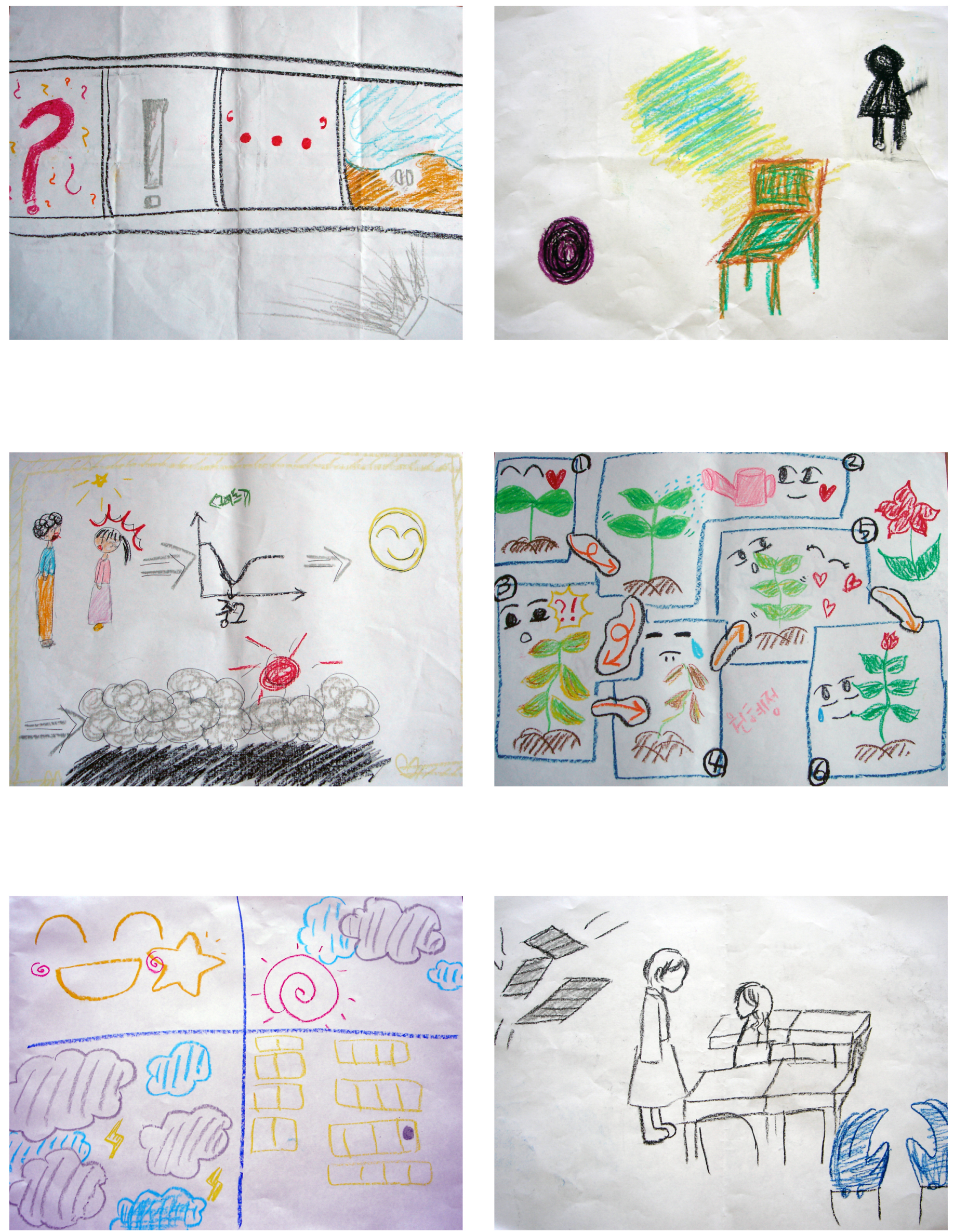


\section{Sitzung: Negative Gefühle zum Selbst}
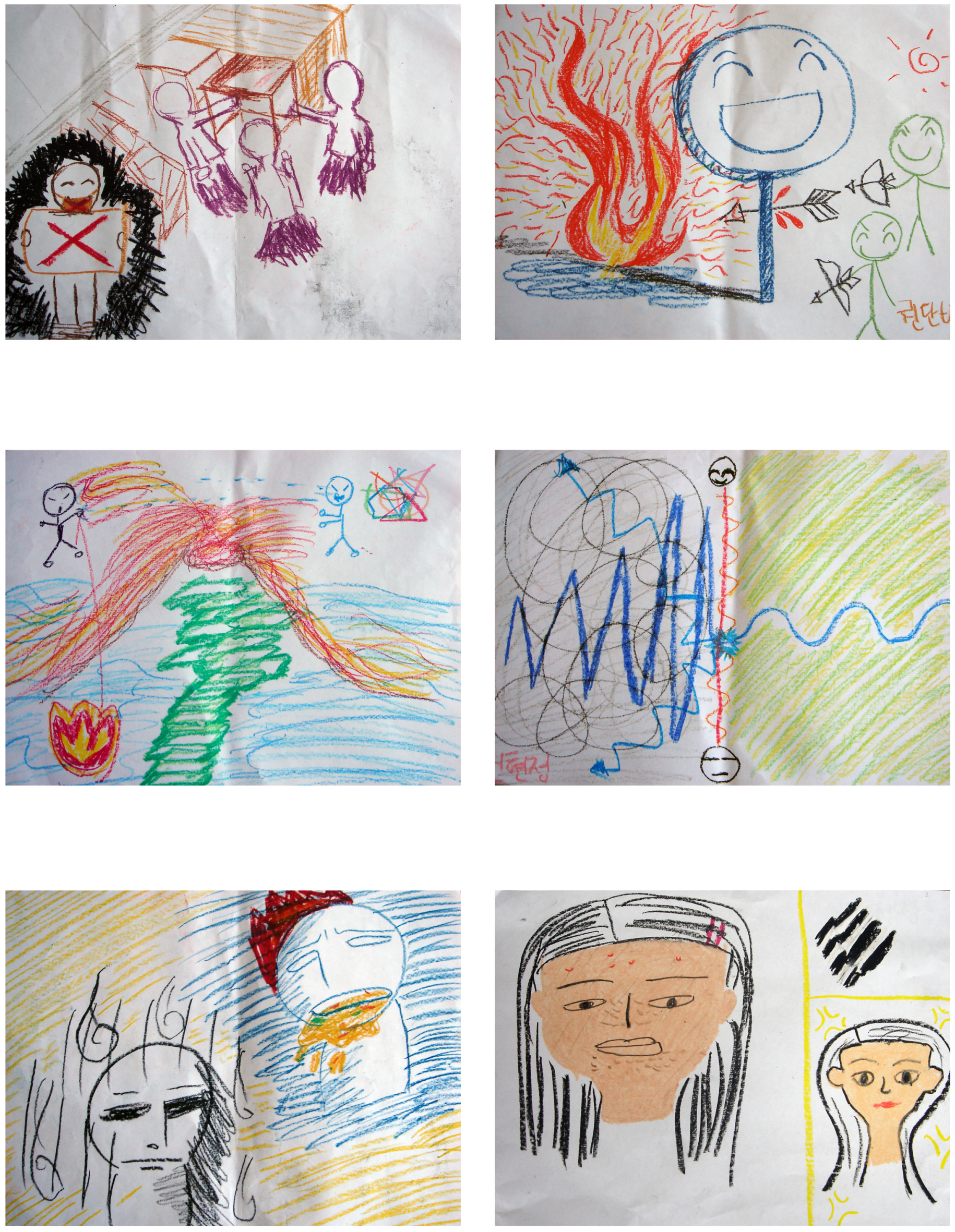
6.Sitzung: Unterbrochen Gefühle zu einer bestimmten Person
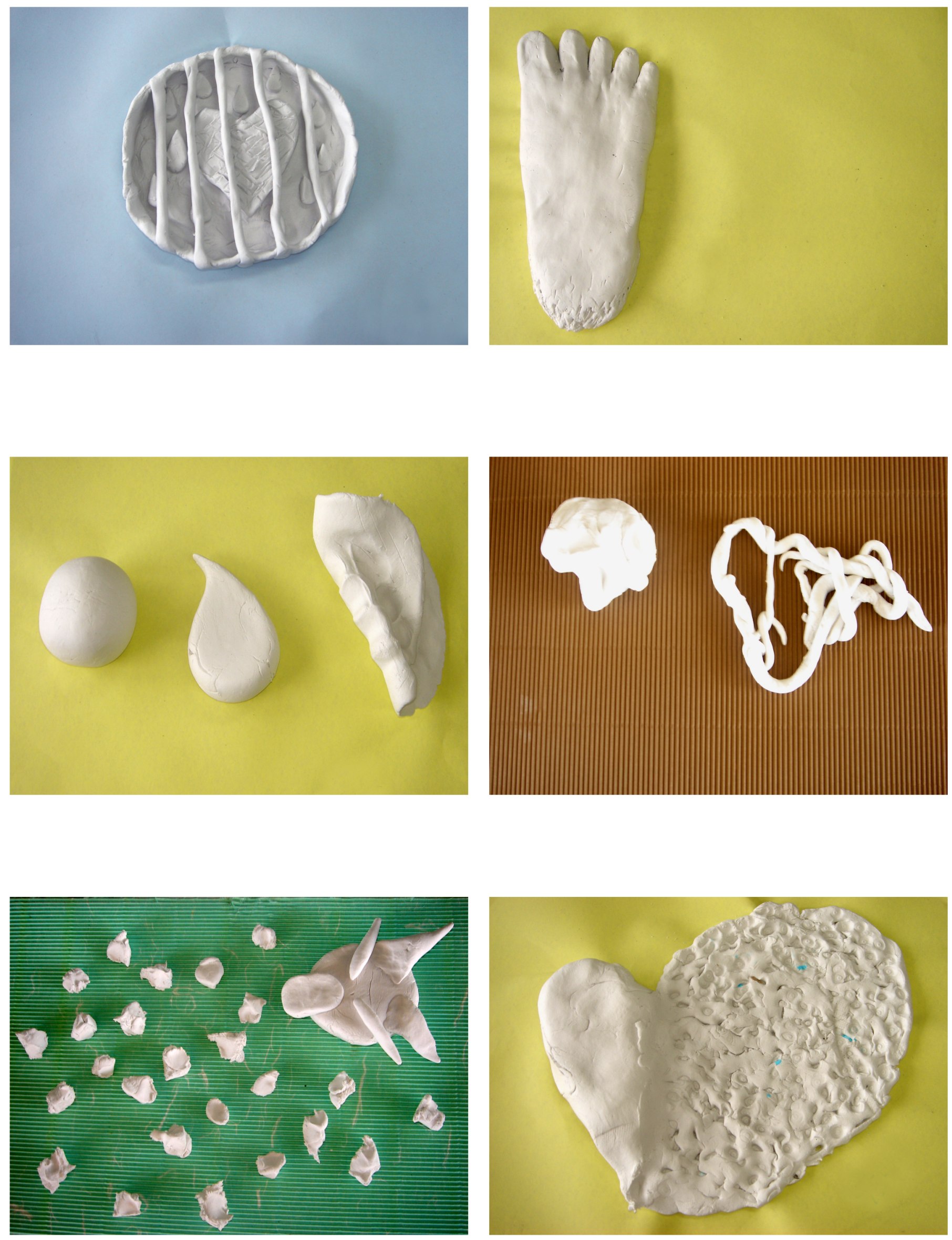


\section{Sitzung: Ein Baum als Ich}
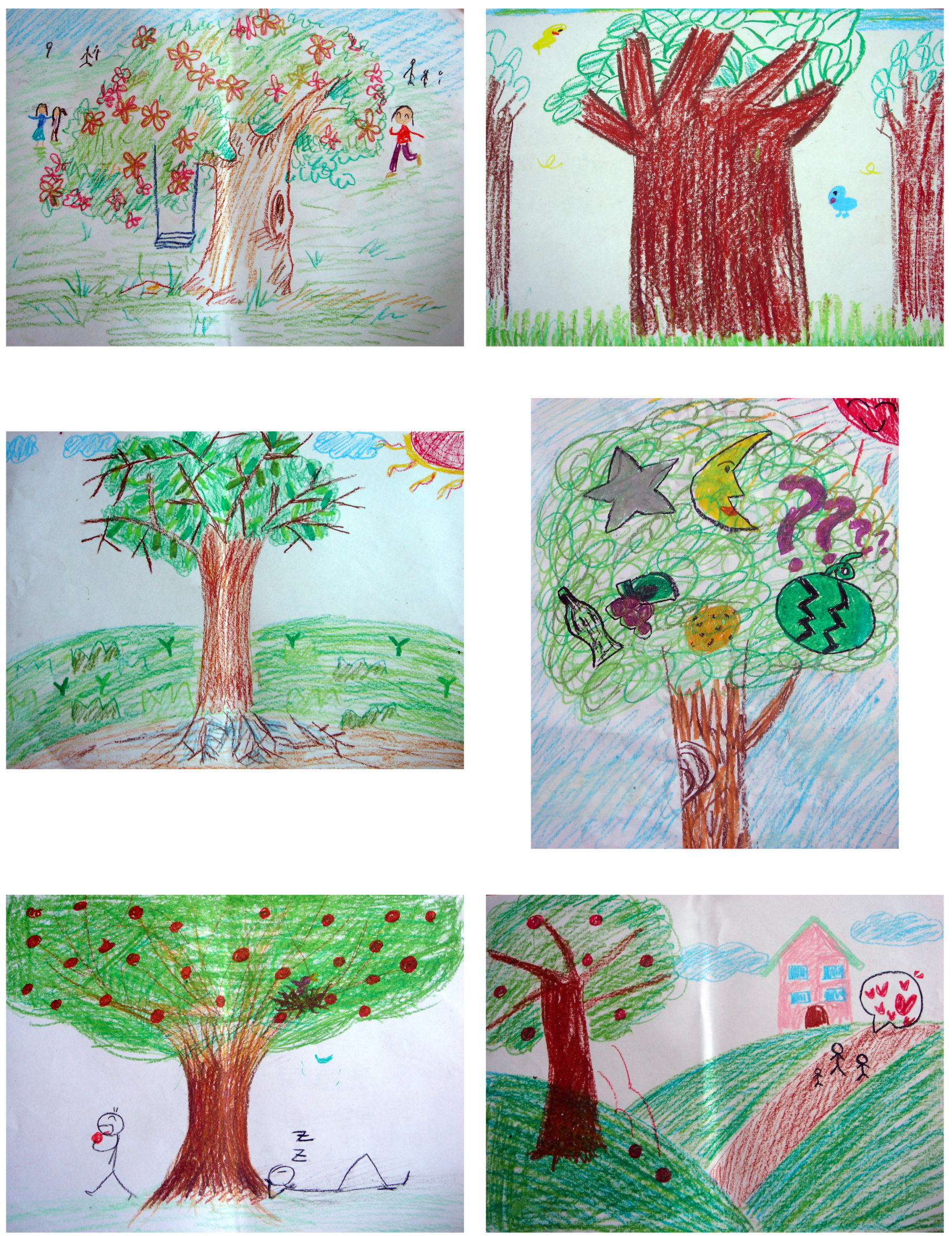
B. Sizungen des Programms im Überblick

\begin{tabular}{|c|c|c|c|c|c|}
\hline Phase & $\mathbf{s}$ & Themen & Hauptmethode & Ziel & Medien \\
\hline Information & 0 & Propädeutik & Einführung & $\begin{array}{l}\text {-Information über Prinzipien und Regeln in der } \\
\text { Gruppenarbeit } \\
\text {-Unterschreiben des schriftlichen Einverständnisses }\end{array}$ & Informationsblätter \\
\hline \multirow[t]{3}{*}{$\begin{array}{l}\text { Schnitt1. } \\
\text { erlebnisorientierte } \\
\text { Exploration }\end{array}$} & 1 & Selbstvorstellung & Vorstellung & $\begin{array}{l}\text { - Kontaktförderung zum Selbst } \\
\text { - Aktivierung der inneren Welt } \\
\text { Schärfung der Wahrnehmnung zum Selbst: v.a.eigene } \\
\text { Bedürfnisse }\end{array}$ & $\begin{array}{l}\text { Die Materialien zur } \\
\text { Selbstvorsetellung }\end{array}$ \\
\hline & 2 & Familie als Lebenswelt & Familie in Tieren & $\begin{array}{l}\text { - Kontakkförderung zum Selbst und zum Anderen } \\
\text { - Förderung der Wahrnehmung der Lebenswelt „Familie“ } \\
\text { - Aktivierung eigener Ressource } \\
\text {-Erkennen der Probleme u. Konflikte in der Familie }\end{array}$ & $\begin{array}{l}\text { Bewegung, } \\
\text { Musik, Malen, } \\
\text { Schreiben }\end{array}$ \\
\hline & 3 & Schule als Lebenswelt & Schulpanorama & $\begin{array}{l}\text { - Kontakkförderung zum Selbst und zum Anderen } \\
\text { - Förderung der Wahrnehmung der Lebenswelt „Schule“ } \\
\text { - Aktivierung eigener Ressource } \\
\text {-Erkennen der Probleme u. Konflikte in der Schule }\end{array}$ & $\begin{array}{l}\text { Bewegung, } \\
\text { Musik, Malen, } \\
\text { Schreiben }\end{array}$ \\
\hline
\end{tabular}




\begin{tabular}{|c|c|c|c|c|c|}
\hline \multirow[t]{4}{*}{$\begin{array}{l}\text { Schnitt } 2 . \\
\text { Aktiver Ausdruck }\end{array}$} & 4 & \multirow[t]{2}{*}{$\begin{array}{l}\text { Erkennen des negativen } \\
\text { Gefühls zum Selbst u. } \\
\text { Ausruck }\end{array}$} & $\begin{array}{l}\text { Gestalten des } \\
\text { negativen Gefühls } \\
\text { zum Selbst }\end{array}$ & $\begin{array}{l}\text { Erkennen und Verstehen negativer Gefühle zum sich } \\
\text { Selbst } \\
\text { - Aktiver Ausdruck der negativen Gefühlen zum sich Selbst } \\
\text { - Förderung der Selbstunterstützung u. Selbstregulation }\end{array}$ & $\begin{array}{l}\text { Malen, Musik, } \\
\text { Schreiben }\end{array}$ \\
\hline & 5 & & $\begin{array}{l}\text { Ausdruck der } \\
\text { negativen Gefühle } \\
\text { zum Selbst }\end{array}$ & $\begin{array}{l}\text { - Erkennen und Verstehen negativer Gefühle zum sich } \\
\text { Selbst } \\
\text { - Aktiver Ausdruck der negativen Gefühlen zum sich Selbst } \\
\text { - Auflösung der Selbstkonflikte im bestimmten } \\
\text { Lebenskontext. } \\
\text { - Förderung des Selbstakzeptanz } \\
\text {-Förderung der Selbstunterstützung u. Selbstregulation }\end{array}$ & $\begin{array}{l}\text { Das Bild, das in } 5 . \\
\text { Sitzung gemalt } \\
\text { wurde, und die } \\
\text { Bilder, die in } 3.4 . \\
\text { Sitzungen gemalt } \\
\text { wurden. }\end{array}$ \\
\hline & 6 & \multirow[t]{2}{*}{$\begin{array}{l}\text { Unterbrochene Gefühle } \\
\text { zu einer bestimmten } \\
\text { Person u. Ausdruck }\end{array}$} & $\begin{array}{l}\text { Gestalten des } \\
\text { Gefühls mit Ton }\end{array}$ & $\begin{array}{l}\text { - Erkennen und Verstehen eigener Gefühle zu einer } \\
\text { bestimmten Person } \\
\text { - Aktiver Ausdruck der negativen Gefühlen gegenüber eine } \\
\text { bestimmte Person } \\
\text { - Förderung der Selbstunterstützung u. Selbstregulation }\end{array}$ & $\begin{array}{l}\text { Bewegung, Musik } \\
\text { Ton, Schreiben }\end{array}$ \\
\hline & 7 & & $\begin{array}{ll}\text { Ausdruck mit } & \text { m der } \\
\text { Tonfigur in } & \\
\text { Lebenswelt } & \end{array}$ & $\begin{array}{l}\text { - Aktiver Ausdruck der negativen Gefühlen gegenüber eine } \\
\text { bestimmte Person } \\
\text { - Auseinandersetzen unerledigter Geschäfte im bestimmten } \\
\text { Lebenskontext. } \\
\text { - Förderung der Selbstbekräftigung } \\
\text { - Förderung der Selbstunterstützung u. Selbstregulation }\end{array}$ & $\begin{array}{l}\text { Tonfigur und die } \\
\text { Bilder, die un } 3.4 . \\
\text { Sitzungen gemalt } \\
\text { wurden. }\end{array}$ \\
\hline
\end{tabular}




\begin{tabular}{|c|c|c|c|c|c|}
\hline & 8 & Ich-Grenzen & $\begin{array}{l}\text { Gestalten eigener } \\
\text { Grenzen }\end{array}$ & $\begin{array}{l}\text { Erkennen und Verstehen durch anschauliche Gestalten } \\
\text { eigener Ich-Grenzen mit der Werken. } \\
\text { - Aktiver Ausdruck der Restsgefühle } \\
\text { - Förderung der Selbstunterstützung u. Selbstregulation }\end{array}$ & $\begin{array}{l}\text { Musik, Bewegung, } \\
\text { die Werke, die bis } \\
\text { jetzt gemacht } \\
\text { wurden. }\end{array}$ \\
\hline \multirow[t]{2}{*}{$\begin{array}{l}\text { Schnitt } 3 . \\
\text { Integration }\end{array}$} & 9 & Ich bin Ich & Ein Baum als Ich & $\begin{array}{l}\text { - Überschau auf eigenem Lebensweg und Lebensziel } \\
\text { - Wiederbelebung eigener Ressource } \\
\text { - Gewinnen der Selbstverantwortung } \\
\text { - Versuch zur Integration, was in der Gruppe erlebt wurde. }\end{array}$ & $\begin{array}{l}\text { Malen, } \\
\text { Musik, } \\
\text { Schreiben, } \\
\text { die Werke, die bis } \\
\text { jetzt gemacht } \\
\text { wurden }\end{array}$ \\
\hline & 10 & Abschied & $\begin{array}{l}\text { Abschiedspiel und - } \\
\text { nehmen }\end{array}$ & $\begin{array}{l}\text { - Lernen Abschied zu nehmen. } \\
\text { - Gewinnen der Selbstverantwortung } \\
\text { - Versuch zur Integration, was in der Gruppe erlebt wurde. }\end{array}$ & \\
\hline Rückmeldung & & Rückmeldung & & $\begin{array}{l}\text { Austausch durch Rückmeldung zur Verbesserung des } \\
\text { Programms }\end{array}$ & \\
\hline
\end{tabular}


C. Prä-Post-Follow-Up Vergleich der Mittelwert und Standardabweichung der erhobenen Variablen

\begin{tabular}{|c|c|c|c|c|c|c|c|c|c|c|c|c|c|c|c|c|}
\hline \multirow{3}{*}{$\begin{array}{c}\text { Fragebogen } \\
\text { Skalen }\end{array}$} & \multicolumn{8}{|c|}{ Versuchsgruppe-GSPD-J } & \multicolumn{8}{|c|}{ Kontrollgruppe } \\
\hline & \multicolumn{2}{|c|}{ Prä } & \multicolumn{2}{|c|}{ Post } & \multicolumn{2}{|c|}{ nach3Monaten } & \multicolumn{2}{|c|}{ nach6Monaten } & \multicolumn{2}{|c|}{ Prä } & \multicolumn{2}{|c|}{ Post } & \multicolumn{2}{|c|}{ nach3Monaten } & \multicolumn{2}{|c|}{ nach6Monaten } \\
\hline & $\mathbf{M}$ & SD & $\mathbf{M}$ & SD & $\mathbf{M}$ & SD & $\mathbf{M}$ & SD & $\mathbf{M}$ & SD & $\mathbf{M}$ & SD & $\mathbf{M}$ & SD & $\mathbf{M}$ & SD \\
\hline BDI & 29,00 & 4,140 & 4,818 & 2,593 & 7,000 & 279 & 10,27 & 2,028 & 29,54 & 3,936 & 30,68 & 3,797 & 31,31 & 4,156 & 30,81 & 3,171 \\
\hline Depressivität & 3,004 & ,314 & 279 & ,222 & ,251 & ,092 & ,308 & ,174 & 3,007 & 293 & 3,084 & 263 & 3,062 & 241 & 3,122 & 202 \\
\hline SCL-90R /GSI & 1,482 & 274 & 622 & ,105 & 643 & ,097 & 654 & ,067 & 1,364 & ,274 & 1,411 & 240 & 1,464 & ,270 & 1,434 & 293 \\
\hline Somatisierung & 2,075 &, 526 & 204 & ,121 & 264 & 174 & ,374 & 179 & 1,849 & ,351 & 1,955 & ,399 & 1,958 & ,363 & 2,041 & ,416 \\
\hline Zwanghaftigkeit & 1,413 & ,323 & 818 & ,288 & 940 & ,125 & 790 & 248 & 1,200 & ,263 & 1,450 & ,252 & 1,740 & ,347 & 1,686 & ,352 \\
\hline Unsicherheit & 2,287 & 685 & 1,171 & ,322 & 1,144 & 367 & 1,221 & 290 & 1,873 & 609 & 1,919 &, 569 & 1,919 &, 516 & 1,878 & ,445 \\
\hline Ängstlichkeit & 1,636 & ,703 & ,900 & 244 & ,945 & 228 & 1,036 & ,315 & 1,500 & 679 & 1,550 & 640 & 1,668 & 646 & 1,654 &, 539 \\
\hline Feinseligkeit & 1,340 & ,396 & 697 & 270 & ,727 & ,301 & ,795 & 280 & 1,174 & 494 & 1,007 & ,335 & 1,143 & ,480 & 1,060 & ,400 \\
\hline Phobische Angst &, 539 & 223 & ,492 & ,226 & ,458 & ,219 &, 543 & 248 & 642 & ,378 & 707 & 251 & 674 & ,344 & 733 & ,385 \\
\hline ParanoidesDenken &, 582 & 275 & 469 & 244 & ,492 & 208 & ,438 & 238 &, 575 & 210 &, 553 & ,242 & ,568 & 279 &, 584 & 251 \\
\hline Psychotizismus & ,463 & 198 & 390 & ,184 & ,377 & ,174 & 381 & , 162 & 450 & 213 & ,477 & ,222 & ,445 & ,217 & ,450 & 197 \\
\hline PWB & 1,722 & ,097 & 3,783 & 110 & 3,465 & 131 & 3,203 & 085 & 1,716 & 132 & 1,712 & 115 & 1,762 & 113 & 1,203 & 085 \\
\hline Selbstakzeptanz & 1,596 & 249 & 3,983 & 212 & 3,795 & 307 & 3,562 & 199 & 1,738 & 188 & 1,670 & 284 & 1,625 & 320 & 1,812 & 143 \\
\hline PositiveBeziehung & 1,668 & 280 & 3,948 & 403 & 3,539 & 341 & 3,409 & ,312 & 1,584 & 264 & 1,688 & ,319 & 1,837 & 254 & 1,643 & 244 \\
\hline Autonomie & 1,635 & 257 & 3,528 & 200 & 3,380 & 274 & 3,022 & 331 & 1,664 & 209 & 1,676 & ,227 & 1,841 & ,325 & 1,716 & ,259 \\
\hline BeherrschungderWelt & 1,761 & ,210 & 3,471 & ,234 & 2,988 & 193 & 2,704 & 157 & 1,915 & 205 & 1,887 & ,269 & 1,858 & ,265 & 1,909 & 182 \\
\hline Lebenssinn & 1,773 & 205 & 3,903 & ,234 & 3,558 & ,224 & 3,331 & 199 & 1,766 & 205 & 1,578 & 287 & 1,708 & ,343 & 1,805 & 209 \\
\hline Wachstum & 1,903 & ,234 & 3,903 & ,217 & 3,551 & 295 & 3,233 & ,219 & 1,619 & 248 & 1,756 & ,285 & 1,705 & ,312 & 1,705 & 269 \\
\hline
\end{tabular}


